\title{
Contacts
}

General information about dala in this document may be obtained from the National Energy Information Center (NEIC). Further information about this document may be obtained from Mary J. Hutzler (202/254-5420), Director of the Electric Power Division, Orfice of Coal, Nuclear, Electric and Alternate Fuels, Energy Information Administration (EIA); and Neal Mocrschel (202,254-5640), Chief of the Data Sys. tems Branch.

This publication is available from the Superintendent of Documents, U.S. Government Printing Office (GPO). Information about purchasing this or other ElA publications may be obtained from the GPO or the NEIC. Questions on energy statistics should be directed to the NEIC by mail, telephone or telecommunications device for the deaf.
Addresses, telephone numbers and hours appear below.

National Energy Information Center, EI-2.31 Energy Information Administration Forrestal Building, Room 1F-048

Washington, DC 20585

(2)2)586-8800

Telecommunications Device for the Deaf, Only, (202)586-1181

Hours: 8:00-5:00, Eastern Time,

Monday through Friday

Superintendent of Documents U.S. Government Printing Office

Washington, DC 20402

(202) 783-3238

Cover Photo

This skyline scene in Dallas, Texas, depicts the consumption of electricity, which provides energy to light homes and shopping centers, and to run factories.

Released for Printing: January 17, 1991

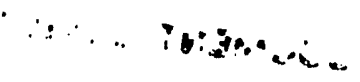




\section{Electric Power Annual}

\section{9}

\section{January 1991}

\section{Energy Information Administration}

Office of Coal, Nuclear, Electric and Alternate Fuels

U.S. Department of Energy

Washington, DC 20585

\section{MATIR}

DISTRIBUTION OF THIS DOCUMENT IS UNLIMITED 


\section{Preface}

The Electric Power Annual presents a summary of electric utility statistics at the national, regional and State levels. The objective of the publication is to provide industry decisionmakers, government policy-makers, analysts and the general public with historical data that may be used in understanding U.S. electricity markets.

\section{Background}

The Electric Power Annual is prepared by the Electric Power Division; Office of Coal, Nuclear, Electric and Alternate Fuels; Energy Information Administration (EIA); U.S. Department of Energy. The 1988 edition was redesigned and expanded to provide a wider perspective of the electric power industry. The 1989 edition continues with this perspective and has been enhanced to include financial statistics on publicly owned electric utilities.

"The Industry At A Glance" section presents a profile of the electric power industry ownership and performance; a review of key statistics for the year; and projections for various aspects of the electric power industry through 2010. Subsequent sections present data on generating capability, including proposed capability additions; net generation; fossil-fuel statistics; electricity sales, revenue and average revenue per kilowatthour sold; financial statistics; environmental statistics; and electric power transactions. In addition, the appendices provide supplemental data on major disturbances and unusual occurrences. Each section contains related text and tables and refers the reader to the appropriate publication that contains more detailed data on the subject matter.

\section{Target Audience}

In the private sector, the majority of users are researchers and analysts and ultimately the policy-and decisionmaking members of electric utility companies. Financial and investment institutions, economic development organizations interested in new power plant construction, special interest groups, lobbyists, electric power associations, and the news media will find the data in the Electric Power Annual useful.
In the public sector, the users include analysts, researchers, statisticians, and other professionals engaged in regulatory, policy, and program areas for Federal, State, and local governments. The Congress and other legislative bodies may also be interested in general trends related to electricity at State and national levels. Much of the data in this report can be used in analytic studies to evaluate new legislation. Public service commissions and other special government groups share an interest in State-level statistics. These groups can also compare the statistics for their States with those of other jurisdictions.

\section{Coverage of Sources}

Data published in the Electric Power Annual are compiled from two statistical forms filed monthly and seven forms filed annually by electric utilities. These forms are: the Form EIA-759, "Monthly Power Plant Report"; the Federal Energy Regulatory Commission (FERC) Form 423, "Monthly Report of Cost and Quality of Fuels for Electric Plants"; the Form EIA-861, "Annual Electric Utility Report"; the Form EIA-860, "Annual Electric Generator Report"; the FERC. Form 1, "Annual Report of Major Electric Utilities, Licensees and Others"; the Form EIA-412, "Annual Report of Public Electric Utilities"; the Form EIA-767, "Steam-Electric Plant Operation and Design Report"; the Department of Energy, Form IE-411, "Coordinated Bulk Power Supply Program"; and the Department of Energy, Form FE-781R, "Annual Report of International Electric Import/Export Data." In addition, the Electric Power Annual also includes data collected on the Form IE-417R, "Electric Power System Emergency Report." A brief description of each of these forms follows. These forms are described in more detail in Appendix C, "Technical Notes."

The Form EIA-159 is used to collect monthly data on net generation; consumption of coal, petroleum, and gas; and end-of-the-month stocks of coal and petroleum for each plant by prime mover. Data are collected from all electric utilities that operate power plants (except those having plants solely on standby), approximately 800 of the 3,250 electric utilities in the United States.

The FERC Form 423 is a restricted-universe census used to collect data on power plants with a fossil-fueled steam-electric nameplate capacity of 50 or more mega- 
watts (approximately 225 electric utilities). This threshold was established by the FERC. Data collected on the FERC. Form 423 include information on fossil fuels regarding quantity, quality, delivered cost, origin, fuel type, supplier, and type of contract under which the fuels are purchased.

The Form ElA.861 is a census of electric utilities in the United States, its territories, and Puerto Rico. The data from the Form EIA-861 that are contained in this publication are for the United States only. The survey is used to collect information on the production, sales, revenue from sales, and trade of electricity from approximately 3,250 electric utilities.

The Form EIA-860 is used to collect data annually from all electric utilities in the United States that operate power plants or plan to operate a power plant within 10 years of the reporting year. Information on electric generators is reported by approximately 900 electric utilities.

The FERC Form 1 is an annual restricted-universe census of the major investor-owned electric utilities having, in each of the last 3 consecutive years, sales or transmission service that exceeds any one or more of the following: (1) 1 million megawathous of total sales, (2) 100 megawat thours of sales for resale, (3) 500 megawatthours of gross interchange out, or (4) 500 megawatthours of wheeling for others (deliveries plus losses). Financial statistics including income; taxes; depreciation and amortization; electric operating revenue; electric maintenance expenses; year-end balance sheets; and, general corporate information are reported on the FERC Form 1 by approximately 180 major investor-owned electric utilities.

The Form EIA-412 is a restricted-universe census used annually to collect accounting, financial, and operating data from publicly owned electric utilities in the United States. Publicly owned electric utilities engaged in the generation, transmission, or distribution of electricity that had 120,000 megawatthours of sales to ultimate consumers and/or 120,000 megawathours of sales for resale for the 2 previous years, as reported on the Form EIA-861, "Annual Electric Utility Report," must submit the Form EIA-412. These criteria result in approx- imately 450 submissions from publicly owned electric utilities.

The Form EIA-767 is a restricted-universe census of all operators of power plants with a fossil-fueled steamelectric existing or planned nameplate capacity of 10 or more megawats. The Form EIA-767 is used to collect data annually on plant operation and equipment design (including boiler, generator, cooling system, flue gas desulfurization units, flue gas particulate collectors, and stack data). Approximately 320 electric utilities provide information regarding approximately 900 power plants on the Form EIA-767.

The Form IE-411 is an annual report supplied to the Department of Energy by the nine North American Electric Reliability Councils. These reports have been compiled from data furnished by the member electric utilities. The Form IE-411 provides the Department of Energy with information on regional plans of electric utilities for a 10-year period and is used for its peak-load information in this publication. The responsibility for collecting these data has been delegated to the Office of International Affairs and Energy Emergencies within the Department of Energy.

The Form IE-417R is used to collect information on major electric utility system emergencies, including the type of emergency, the utility and area affected, the date and time of the event, a description of the event, and expected time of restoration of service. The responsibility for collecting these data has been delegated to the Office of International Affairs and Energy Emergencies within the Department of Energy.

The Form FE-781R is used to collect data annually from holders of Export Authorizations and Presidential Permits (subject to the authority of Part II, Federal Power Act) engaged in the export of electric energy across the intermational borders of the United States with Canada and Mexico. The information collected on the Form FE-781R includes the volume of energy received by and/or delivered to holders of Export Authorizations and Presidential Permits, and the costs and revenue associated with these transactions. The responsibility for collecting these data has been delegated to the Office of Furis, Fossil Energy, within the Department of Enersy. 


\section{Contents}

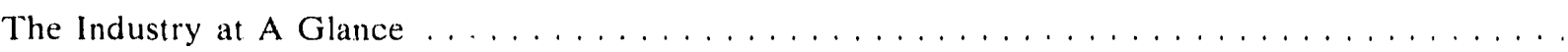

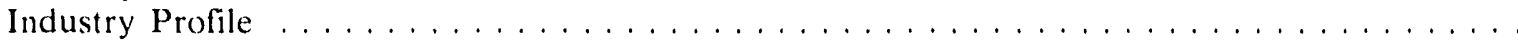

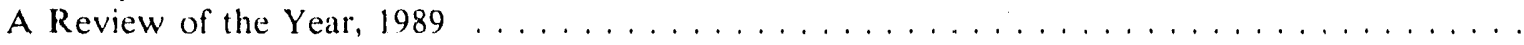

Outlook Through 2010

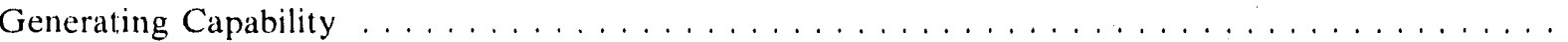

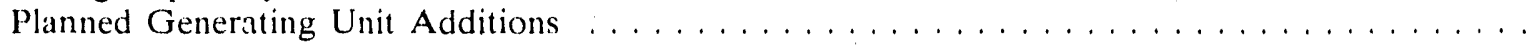

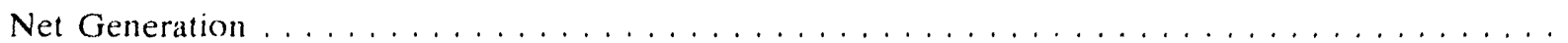

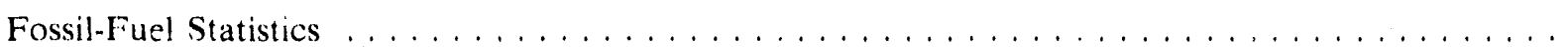

Retail Sales and Revenue $\ldots \ldots \ldots \ldots \ldots \ldots \ldots \ldots \ldots \ldots \ldots \ldots \ldots \ldots \ldots \ldots$

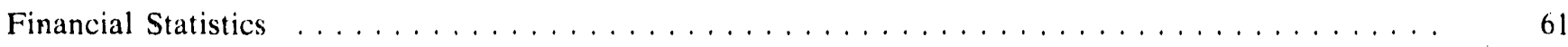

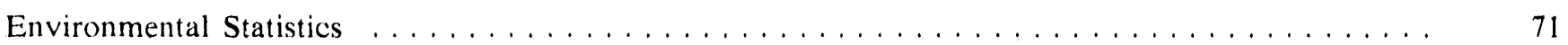

Electric Power Transactions $\ldots \ldots \ldots \ldots \ldots \ldots \ldots \ldots \ldots \ldots \ldots \ldots \ldots \ldots \ldots \ldots \ldots$

Appendices

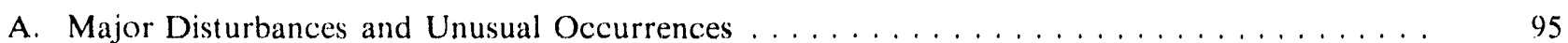

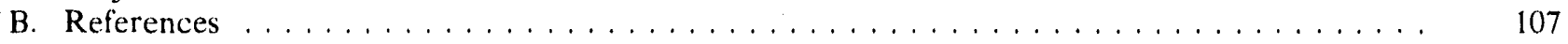

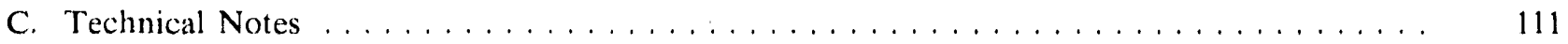

Glossary . . . . . . . . . . . . . . . . . . . . . . . . . . . . . . . . . 127 


\section{Tables}

1. Electric Power Summary Statistics for the United States, 1989 and $1988 \ldots \ldots$

2. Electric Power Projections for the United States Through 2010 . . . . . . . . . . . . . . .

3. Generating Capability by Prime Mover and Primary Energy Source, 1986-1989 . . . . . . .

4. Summary of Capability Additions and Retirements, and Total Operable Capability by

Energy Source, 1989

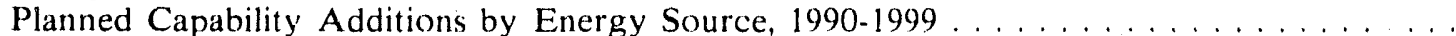

6. Generating Capability by Census Division and State, 1989 and $1988 \ldots \ldots \ldots \ldots$

7. Coal-Fired, Nuclear, Hydroelectric, and Other Generating Capability by Census Division

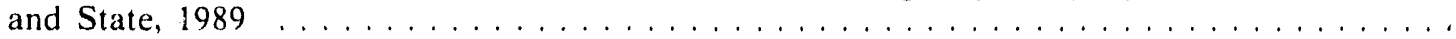

8. Petroleum-, Gas-, and Dual-Fired Steam Generating Capability by Census Division and

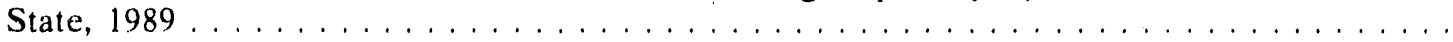

9. Petroleum-, Gas-, and Dual-Fired Gas Turbine/Internal Combustion Generating Capability by Census Division and State, $1989 \ldots \ldots \ldots \ldots \ldots \ldots \ldots \ldots \ldots$

10. Planned Capability Additions by Energy Source and North American Electric Reliability Council Region, Alaska, and Hawaii, 1990-1999 . . . . . . . . . . . . . . . . . . . . .

11. Planned Capability Additions by Energy Source, Census Division, and State, 1990-1999 . . .

12. Net Generation by Energy Source, $1985-1989 \ldots \ldots \ldots \ldots \ldots \ldots$

13. Net Generation by Selected Prime Mover, Census Division, and State, 1989 and $1988 \ldots \ldots$

14. Net Generation by Energ', Source, Census Division, and State, 1989 and 1988 . . . . . . . .

15. Petroleum-Fired Net Generation by Selected Prime Mover, Census Division, and State, 1989 and 1988

16.

Gas-Fired Net Generation by Selected Prime Mover, Census Division, and State, 1989 and

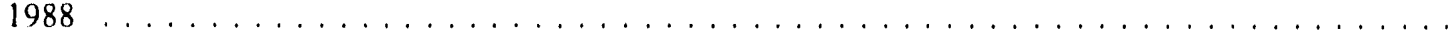

17. Consumption of Fossil Fuels, and Stocks of Coal and Petroleum, 1985-1989 .........

18. Receipts and Average Delivered Cost of Fossil Fuels, 1985-1989 . . . . . . . . . . . . . .

19. Consumption of Fossil Fuels by Census Division and State, 1989 and $1988 \ldots \ldots \ldots$

20. Petroleum Consumption by Selected Prime Mover, Census Division, and State, 1989 and 1988

22. Coal and Petroleum Stocks by Census Division and State, as of December 31, 1989 and 1988

24. Average Delivered Cost of Fossil-Fuel Receipts by Census Division and State, 1989 and

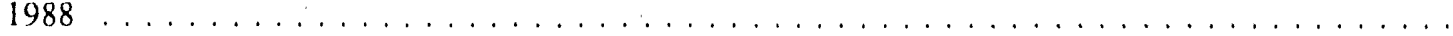

Sales to Ultimate Consumers and Associated Revenue by Sector, 1985-1989 . . . . . . . . Average Revenue per Kilowatthour by Sector, 1985-1989

Sales to Ultimate Consumers by Sector, Census Division, and State, 1989 and $1988 \ldots \ldots$

28. Number of Ultimate Consumers by Sector, Census Division, and State, 1989 and 1988 . . . .

Average Revenue per Kilowatthour by Sector, Census Division, and State, 1989 and $1988 \ldots$ Composite Statement of Income for Selected Investor-Owned Electric Utilities, 1987-1989 . . Composite Balance Sheet for Selected Investor-Owned Electric Utilities, 1987-1989 . . . . . . Composite Financial Indicators for Selected Investor-Owned Electric Utilities, 1987-1989 . . Revenue and Expense Statistics for Selected Investor-Owned Electric Utilities, 1987-1989 .. Revenue and Expense Percentages for Selected Investor-Owned Electric Utilities, 1987-1989 Composite Statement of Income for Selected Publicly Owned Electric Utilities, 1989 . . . . Composite Balance Sheet for Selected Publicly Owned Electric Utilities, $1989 \ldots \ldots$. . . . . . Composite. Financial Indicators for Selected Publicly Owned Electric Utilities, $1989 \ldots \ldots$ Revenue and Expense Statistics for Selected Public ly Owned Electric Utilities, $1989 \ldots \ldots$. . . Emissions from Fossil-Fueled Steam-Electric Generating Units, 1985-1989

Number and Capacity of Fossil-Fueled Steam-Electric Generators for Plants with

Environmental Equipment, 1985-1989

Emissions from Fossil-Fueled Steam-Electric Generating Units by Census Division and

State, 1989 and 1988

43. Emissions from Fossil-Fueled Steam-Electric Generating Units by Fossil Fuel, Census

Division, and State, 1989 
44. Number and Capacity of Coal-Fired Steam-Electric Generators for Plants with Environmental Equipment by Census Division and State, $1989 \ldots \ldots \ldots \ldots \ldots$

45. Number and Capacity of Petroleum- and Gas-Fired Steam-Electric Generators for Plants with Environmental Equipment by Census Division and State, 1989 . . . . . . . . . . . .

46. Average Quality of Fossil Fuels Burned by Census Division and State, 1989 and 1988 . . .

47. Average Flue Gas Desulfurization Costs by Census Division and State, 1986-1989 . . . . . .

48. Energy Account Balance of Electricity, $1987-1989 \ldots \ldots \ldots \ldots \ldots$

49. Net Generation by North American Electric Reliability Council Region, Alaska, and

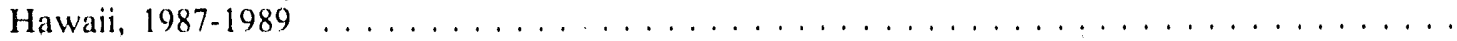

50. Sales to Ultimate Consumers by Sector, North American Electric Reliability Council Region, Alaska, and Hawaii, $1987-1989 \ldots \ldots \ldots \ldots \ldots \ldots \ldots \ldots$

51. Generating Capability by North American Electric Reliability Council Region, Alaska, and

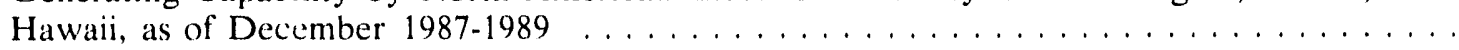
Noncoincidental Peak Load by North American Electric Reliability Council Region,

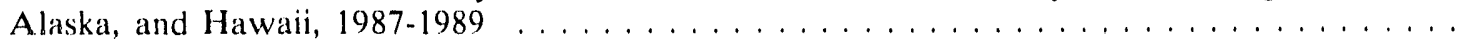

53. Utility Receipts by North American Electric Reliability Council Region, Alaska, and Hawaii, $1987.1989 \ldots \ldots \ldots \ldots \ldots \ldots \ldots \ldots \ldots \ldots \ldots$

54. Utility Deliveries by North American Electric Reliability Council Region, Alaska, and Ha-

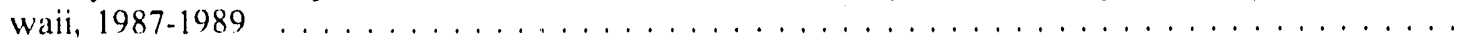

55. Utility Net Energy Flow by North American Electric Reliability Council Region, Alaska,

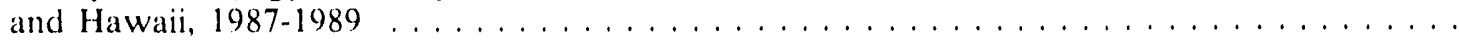
Utility Purchases of Nonutility Generated Electricity by North American Electric

Reliability Council Region, Alaska, and Hawaii, $1987-1989 \ldots \ldots \ldots \ldots$. . . . . . . . . . . . . .
Net Imports by North American Electric Reliability Council Region, Alaska, and Hawaii,

57. Net Imports by North American Electric Reliability Council Region, Alaska, and Hawaii,

A1. Major Disturbances and Unusual Occurrences, $1989 \ldots \ldots \ldots \ldots \ldots$

C1. Comprarison of Prior Versus Final Published Data at the National Level, $1989 \ldots \ldots$. . . . . .

C2. Average Heat Content of Fossil-Fuel Receipts by Census Division and State, 1989 . . . . .

C3. Sulfur Dioxide, Nitrogen Oxide; and Carbon Dioxide Emission Factors . . . . . . . . . . .

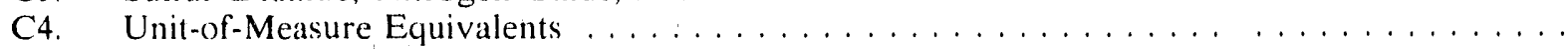




\section{Illustrations}

Page

1. Generating Capability by Class of Ownership, $1989 \ldots \ldots \ldots \ldots$

2. Share of Generating Capability by Class of Ownership, $1989 \ldots \ldots \ldots$

3. Net Generation and Sales to Ultimate Consumers by Class of Ownership, 1989 . . . . . . . . . .

4. Share of Net Generation and Sales to Ultimate Consumers by Class of Ownership, 1989 . . 4

5. Number of Electric Utilities by Class of Ownership, $1989 \ldots \ldots \ldots$

6. Number of Ultimate Consumers Served by Class of Utility Ownership, 1989 . . . . . . . . . . 5

7. Revenue From Sales to Ultimate Consumers by Class of Ownership, 1989 . . . . . . . . . . . . 6

8. Share of Revenue From Sales to Ultimate Consumers by Class of Ownership, 1989 . . . . . 6

9. Share of Sales for Resale by Class of Ownership, $1989 \ldots \ldots \ldots \ldots$

10. Share of Revenue from Sales for Resale by Class of Ownership, $1989 \ldots \ldots$

11. Hydroelectric Net Generation and Percent of Normal Precipitation by Census Division,

12. Sales to Ultimate Consumers by Sector, $1989 \ldots \ldots \ldots \ldots \ldots \ldots$

13. Share of Sales to Ultimate Consumers by Sector, $1989 \ldots \ldots \ldots \ldots$

14. Average Revenue per Kilowathour by Sector, $1989 \ldots \ldots \ldots \ldots \ldots$

15. Net Generation by Energy Source, $1989 \ldots \ldots \ldots \ldots \ldots$

16. Share of Net Generation by Energy Source, $1989 \ldots \ldots \ldots \ldots$

17. Monthly Net Generation, 1989 and $1988 \ldots \ldots \ldots \ldots \ldots$

18. Generating Capability by Energy Source, $1989 \ldots \ldots \ldots \ldots \ldots$

19. Share of Generating Capability by Energy Source, $1989 \ldots \ldots \ldots \ldots$

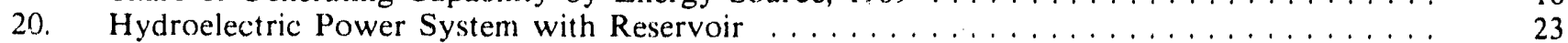

21. The Revenue Dollar from Operations for Selected Investor-Owned Electric Utilities, 1989 . . 65

22. Share of Electric Operation Expenses for Selected Investor-Owned Electric Utilities, 1989 . . 66

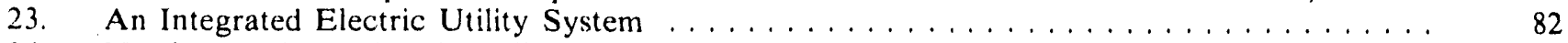

24. North American Electric Reliability Council Region Map for the United States . . . . . . 


\section{The Industry at A Glance}

Electricity is vital to virtually every aspect of the national economy. U.S. dependency on electricity is expected to expand in the future. Consequently, the role of the industry charged with the responsibility for producing electric power and maintaining electric power service in the United St: tes is significant.
The first section of this chapter provides a graphically illustrated profile of the electric power industry. The second section of this chapter continues that descriptive profile by summarizing pertinent statistics on various aspects of the industry for the year. The third, and last section, of this chapter provides projections of pertinent statistics related to the electric power industry through 2010.

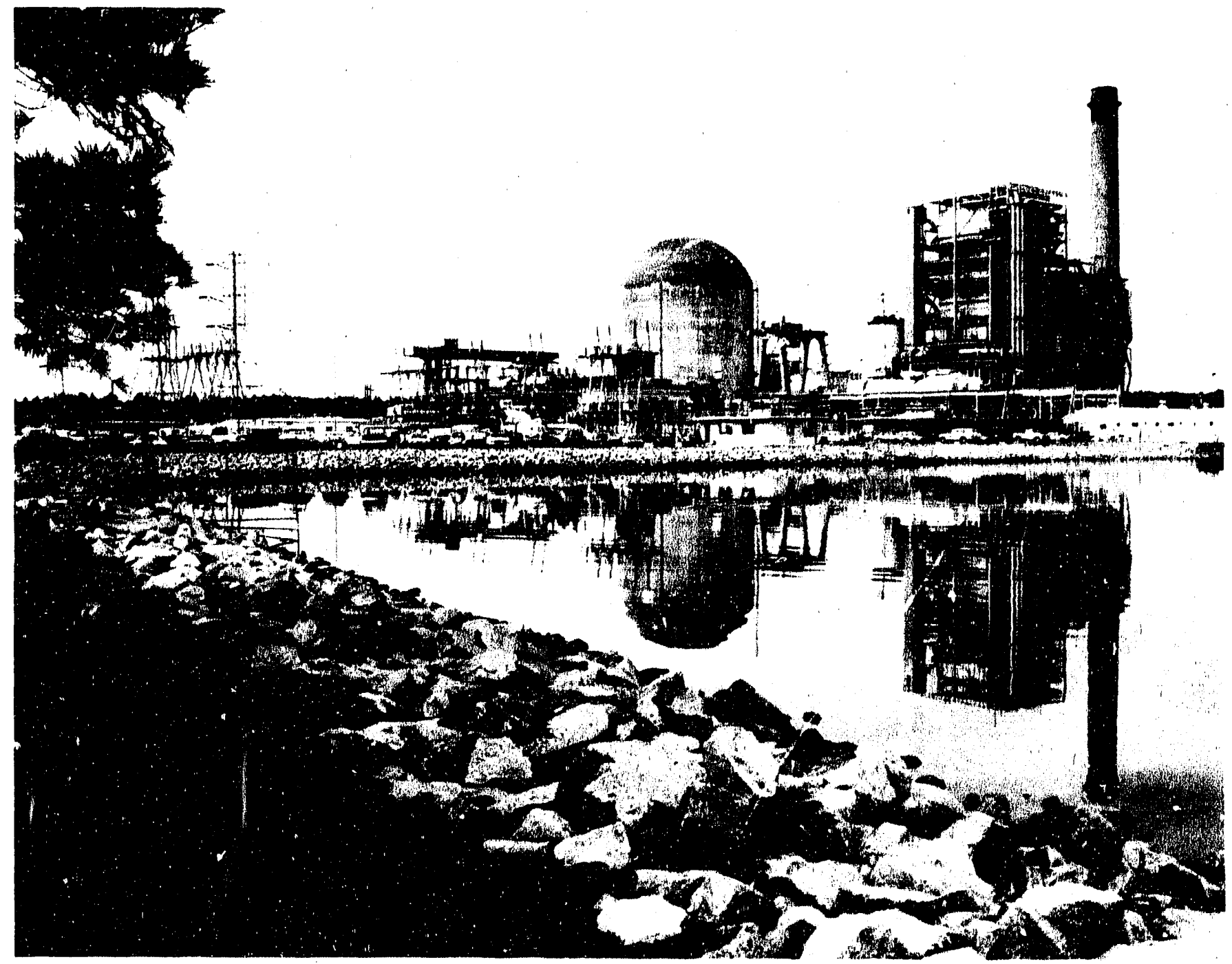

The H.B. Robinson Nuclear Power Plant is owned by the Carolina Power and Light Company. This 66.5-megawatt pressurized water reactor, located near Hartsville, South Carolina, was the first commercial-size nuclear plant in the Southeast. 


\section{Industry Profile}

The U.S. electric power industry is a combination of electric utilities and nonutility power producers.' The electric utility industry is comprised of investor-owned, publicly owned, Federal, and cooperative electric util. ities. Historically, investor-owned electric utilities have been most successful in serving large consolidated markets where economies-of-scale afforded the lowest prices. However, publicly owned, Federal, and cooperative electric utilities all have a role in producing, transmitting, and distributing electricity.

Investor-Owned Electric Utilities. Like all private businesses, the fundamental objective of a private (investor-owned) utility is to produce a return for investors. The investor-owned electric utility either distributes its profits to stockholders as dividends or reinvests these profits. It is granted a service monopoly in certain geographic areas and is obliged to serve all consumers. As franchised monopolies, these electric utilities are regulated and required to charge reasonable prices, to charge comparable prices to similar classifications of consumers, and to give consumers acc is to services under similar conditions. Most investor-owned electric utilities are' operating companies that provide basic services for the generation, transmission, and distribution of electricity. The majority of investor-owned electric utilities perform all three functions. Investorowned electric utilities operate in all States except Nebraska. The electric utilities in Nebraska consist primarily of municipal systems and public power districts. Hawaii is the only State in which all electricity generation is supplied by investor-owned electric utilities. Investor-owned electric utilities currently account for more than 75 percent of all U.S. electric utility generating capability, generation, sales, and revenue.

Publicly Owned Electric Utilities. Publicly owned electric utilities are nomprofit local government agencies established to serve their commurities and nearby consumers at cost, returning excess funds to the consumer in the form of community contributions, economic and efficient facilities, and lower rates. Publicly owned electric utilities (which number approximately 2,000 ) include municipals, public power districts, State authorities, irrigation districts, and other State organizations. Most municipal electric utilities simply distribute power, although some larger ones produce and transmit electricity as well. They obtain their financing from municipal treasuries and from revenue bonds secured by proceeds from the sale of electricity. Public power districts and projects are concentrated in $\mathrm{Ne}$ braska, Washington, Oregon, Arizona, and California. Voters in a public power district elect commissioners or directors to govern the district, independent of any municipal government. State authorities, like the
Power Authority of the State of New York or the South Carolina Public Service Authority, are agencies of their respective State governments. Irrigation districts may have still other forms of organization. In the Salt River Project in Arizona, for ex mmple, votes for the board of directors are apportioned according to the size of landholdings.

Federal Electric Utilities. Power produced by Federal electric utilities is not generated for profit. Most of the electricity produced is sold to publicly owned and cooperative electric utilities, and other nonprofit entities as required by law. The Federal Government is primarily it producer and wholesaler of electricity. These wholesale producers include the U.S. Army Corps of Engineers, the Bureau of Indian Affairs, the Bureau of Reclamation, and the International Water and Boundary Commission. The power marketing administrations of the U. S. Department of Energy include Bonneville, Southeastern, Southwestern, and Western Area. The Alaska Power Administration and the Tennessee Valley Authority both generate and market electricity. These power marketing administrations operate in all States except those in the Northeast, the Upper Midwest, and Hawaii.

Cooperative Electric Utilities. Cooperative electric utilities are owned by their members and are established to provide electricity to those members. These electric utilities operate in rural areas with a lower concentration of consumers as these areas are not economical for investor-owned electric utilities. They are incorporated under State law and are usually directed by an elected board of directors, which in turn selects a manager. The Rural Electrification Administration, th: National Rural Utilities Cooperative Finance Corporation, the Federal Financing Bank, and the Bank for Cooperatives are the most important sources of debt financing for cooperatives. Ther are approximately 960 cooperative electric utilities currently doing business in 46 States. Cooperative electric utilities do not operate in Connecticut, Hawaii, Massachusetts, Rhode Island, or in the District of Columbia.

\section{Presentation of Data}

The following graphic presentation on the electric utility industry by class of ownership includes: generating capability; net generation and sales of electricity; and the number of utilities, generators, and ultimate consumers. These data are collected and compiled from various sources as indicated in the Preface. Data on generating capability and net generation of electricity are presented for all electric utilities that produce power in the United States; data on electricity sales and revenue cover all electric utilities that have retail sales in the United States. 
Figure 1. Generating Capability by Class of Ownership, 1989

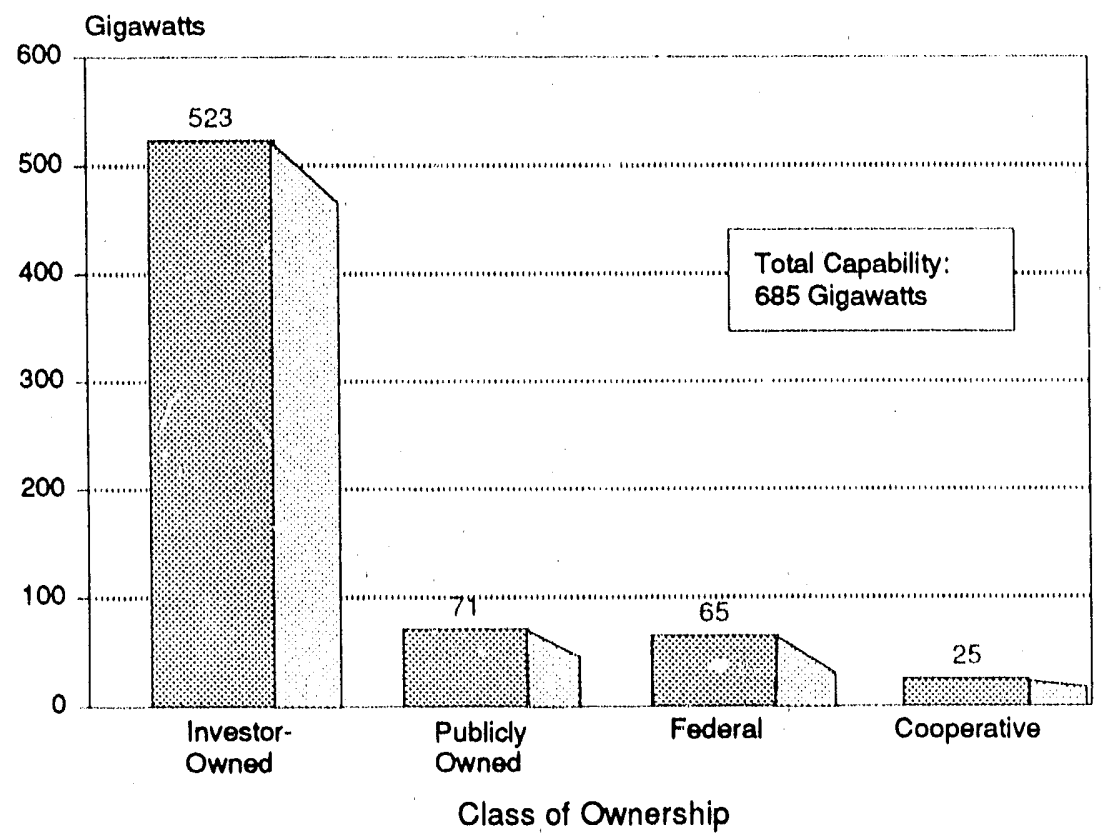

Notes: Generating capability is net summer capability. Totals may not equal sum of components because of independent rounding.

Source: Energy Information Administration, Form EIA-860, "Annual Electric Generator Report."

Figure 2. Share of Generating Capability by Class of Ownership, 1989

Total Capability: 685 Gigawatts

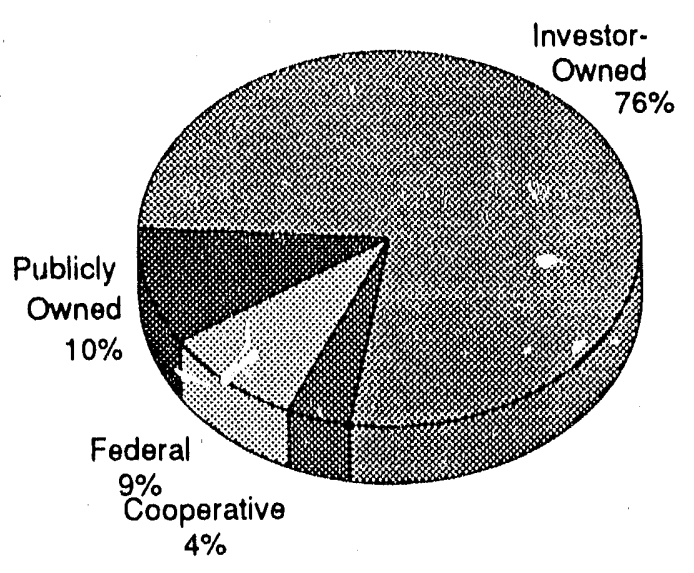

Notes: Generating capability is net summer capability. Totals may not equal sum of components because of independent rounding.

Source: Energy Information Administration, Form EIA-860, "Annual Electric Generator Report." 
Figure 3. Net Generation and Sales to Ultiınate Consumers by Class of Ownership, 1989

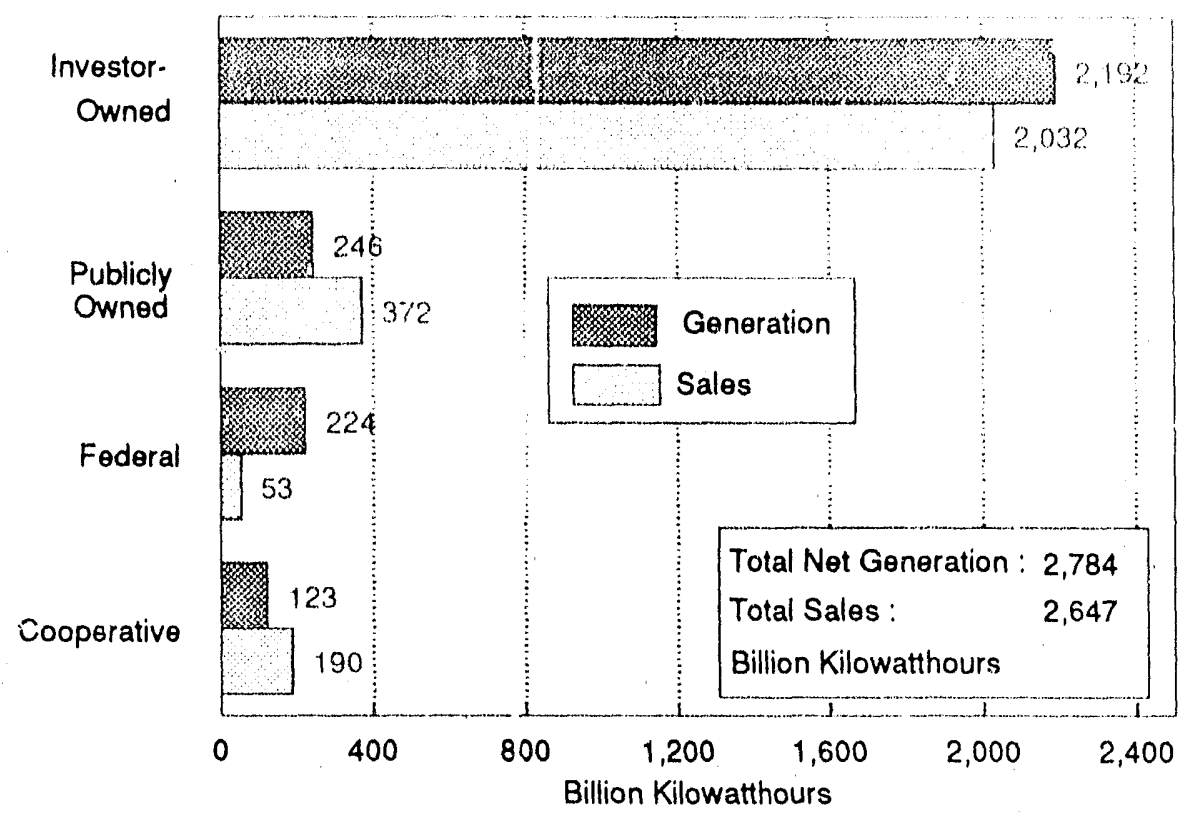

Notes: Total nonutility generation of electricity, which is not included in the generation data, was estimated to be 193 billion kilowatthours in 1989. Nonutility sales of electricity to electric utilities (for resale to ultimate corisumers) were 90 billion kilowatthours and are included in the sales data in this graph. Totals may not equal sum of components because of independent rounding.

Sources: Energy Information Administration, Utility generation: Form ElA-759, "Monthly Power Plant Report"; Nonutility generation: Annual Outlook for U.S. Electric Power 1990, DOE/EIA-0474(90) (Washington, DC, June 1990); Sales: Form EIA-861, "Annual Electric Utility Report."

Figure 4. Share of Net Generation and Sales to Ultimate Consumers by Class of Ownership, 1989
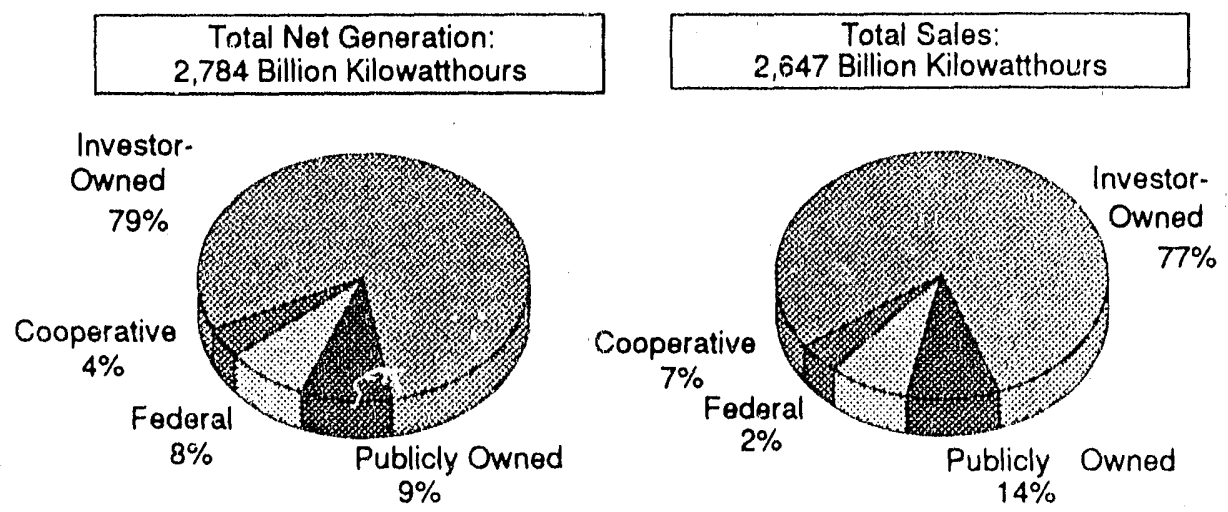

\section{Generation}

\section{Sales}

Notes: See notes for Figure 3. Totals may not equal sum of components because of independent rounding.

Sources: Energy Information Administration, Utility generation: Form ElA-759, "Monthly Power Plant Report"; Nonutility generation: Annual Outlook for U.S. Eloctric Power 1990, DOE/EIA-0474(90) (Washington, DC, June 1990); Sales: Form ElA-861, "Annual Electric Utility Report." 
Figure 5. Number of Electric Utilities by Class of Ownership, 1989

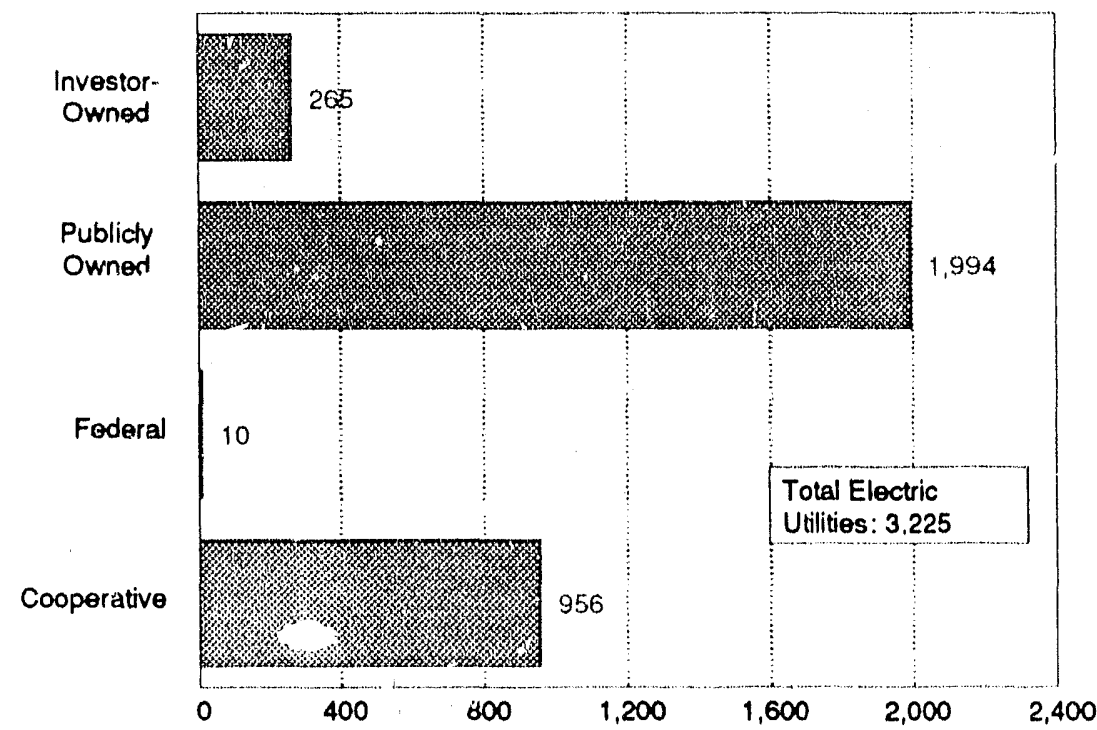

Source: Energy Information Adminstration, Form ElA-861, "Annual Flectric Utility Report."

Figure 6. Number of Ultimate Consumers Served by Class of Utility Ownershin, 1985

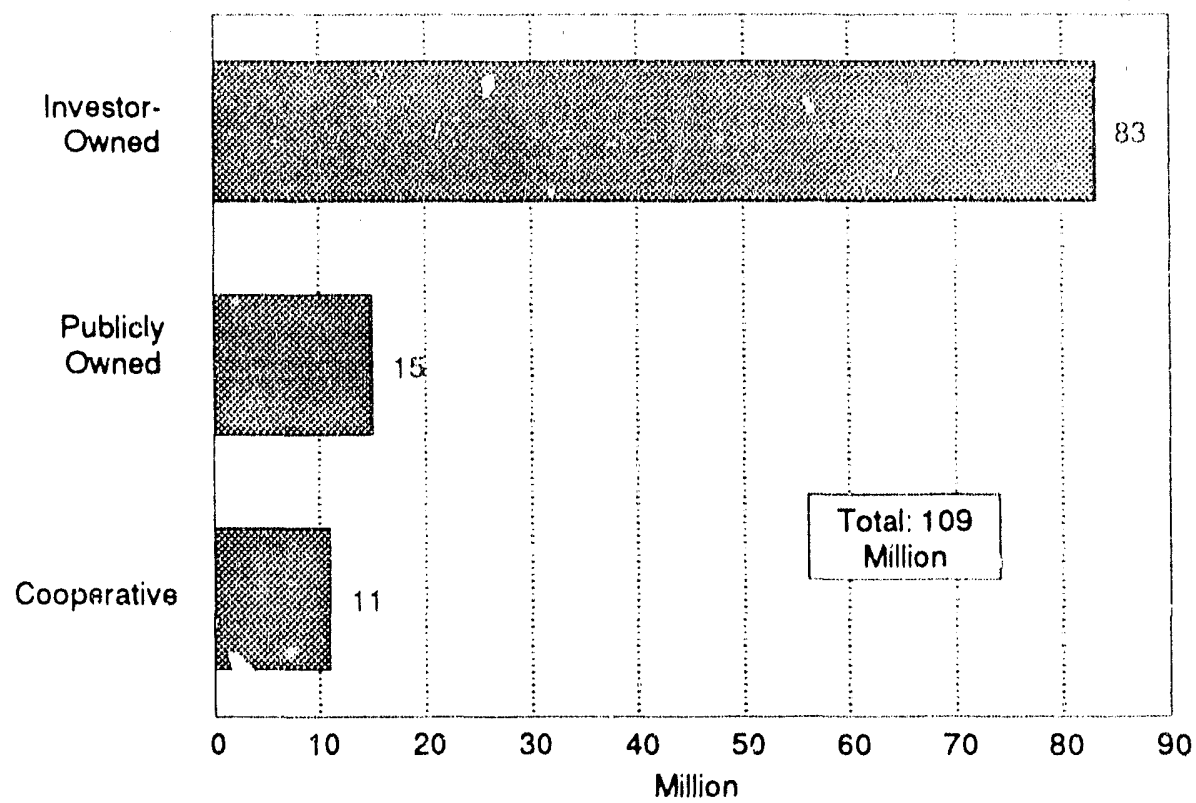

Notes: The number of ultimate consumers served by Federal electric utilities is less than 1 million. The number of ultimate consumers is an average of the number of consumers at the close of each month. Totals may not equal sum of components because of independent rounding.

Source: Energy Information Administration, Form ElA-861, "Annual Electric Utility Report." 
Figure 7. Revenue From Sales to Ultimate Consumers by Class of Ownership, 1989

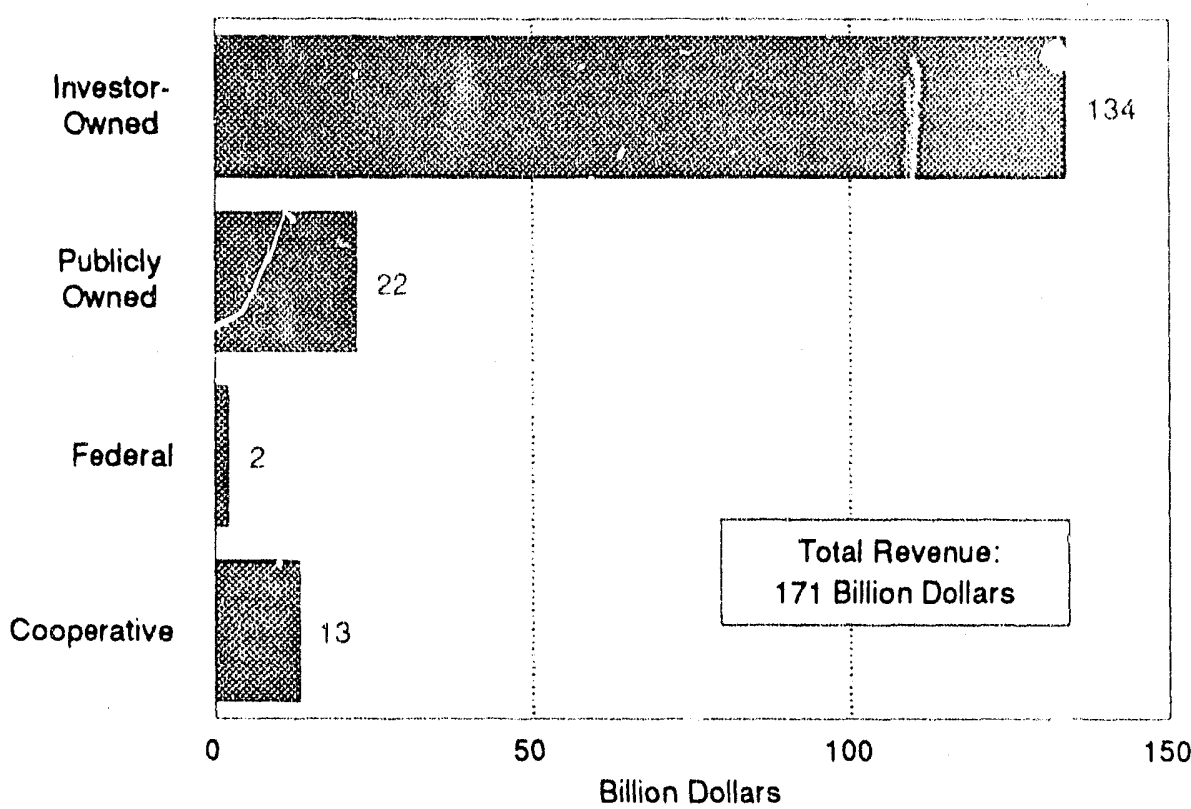

Note: Totals may not equal sum of components because or independent rounding.

Source: Energy Information Administration, Form ElA-861, "Annual Electric Utility Ruport."

Figure 8. Share of Revenue From Sales to Ultimate Consumers by Class of Ownership, 1989

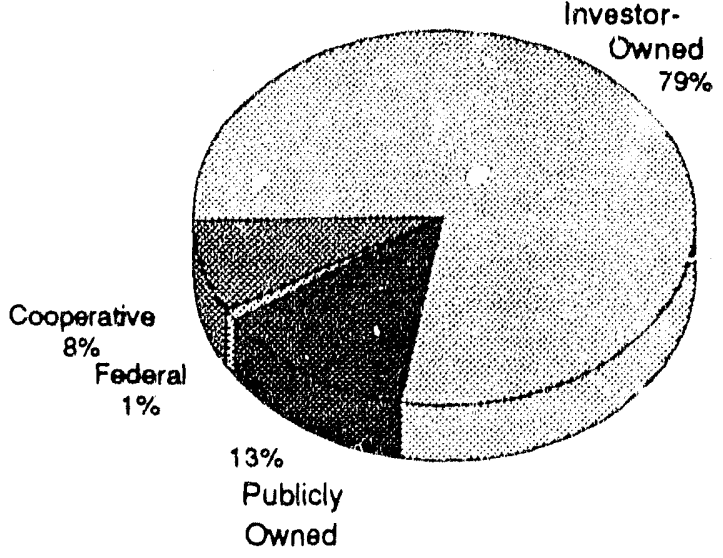

Note: Totals may not equal sum of components because of independent rounding.

Source: Energy Infurmation Administration, Form ElA-861, "Annual Electric Utility Report." 
Figure 9. Share of Sales for Resale by Class of Ownership, 1989

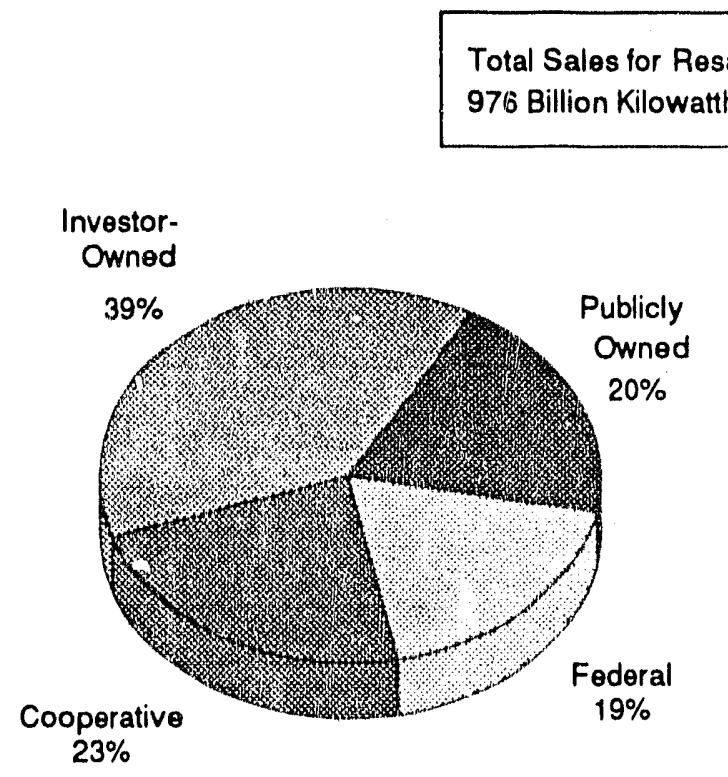

Notes: Totals may not equal sum of components because of independent rounding.

Source: Energy Informatior. Adminstration, Form ElA-861, "Annual Elecric Utility Report."

Figure 10. Share of Revenue from Sales for Resale by Class of Ownership, 1989

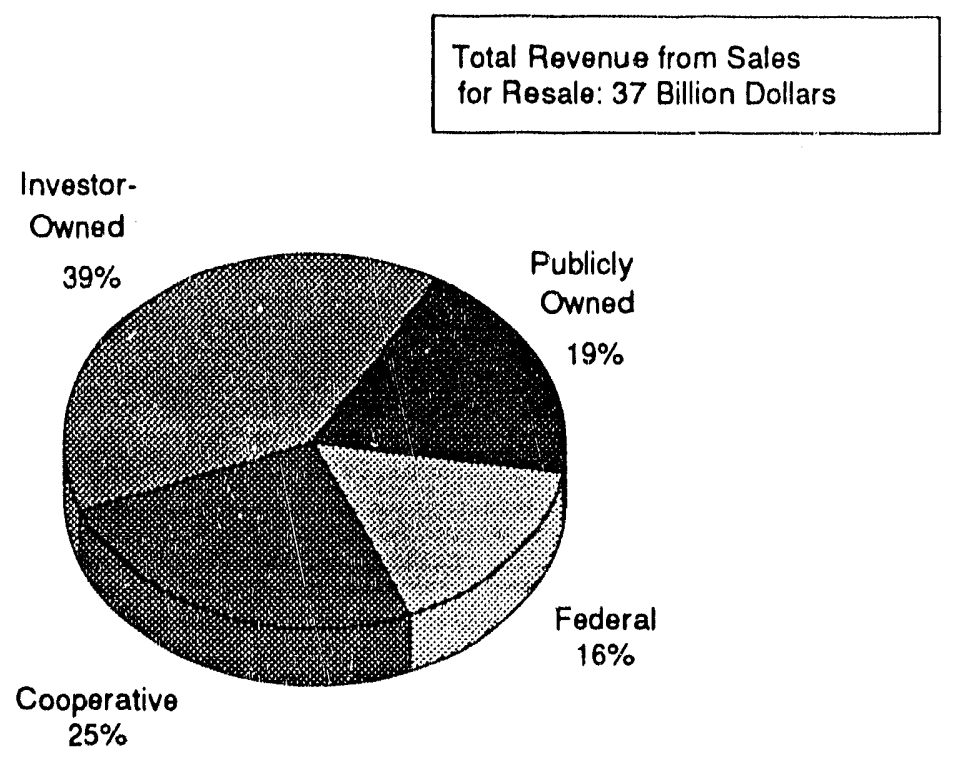

Notes: Totals may not equal sum of components because of independent rounding.

Source: Energy Information Administration, Form EIA-861, "Annual Electric Utility Report." 


\section{A Review of the Year, 1989}

The year 1989 was the seventh consecutive year of economic expansion in the United States; however, the rate of that expansion was substantially less than in prior years. 2 A 2.5-percent increase in real gross national product in 1989 compared with a 4.5-percent increase in 1988 was indicative of this slower growth rate. In a similar fashion, while demand for electricity in 1989 surpassed that in 1988, the rate of its growth slowed considerably. Generally mild weather (except during December) and temperate economic conditions during 1989 contributed to the slower growth during the year.

\section{Sales Are Moderate}

Total U.S. sales of electricity rose by 2.7 percent in 1989 from 1988 (Table 1). A record for total sales of electricity was set in 1989 at 2,647 billion kilowatthours. However, electricity sales in all the major electricity-consuming end-use sectors grew less in 1989 than in 1988 (Table 25). In 1989, residential sales of electricity ( 906 billion kilowatthours) reflected a 1-percent increase compared with the 5-percent increase reported in 1988. This was the smallest rate of growth in the residential sector in the past 5 years. Sales of electricity in the commercial sector rose 4 percent in 1989, the largest increase for all end-use sectors for the second consecutive year. Industrial sales of electricity increased by only 3 percent in 1989 compared with the 4-percent increase in 1988. The lower level of growth for sales of electricity in the industrial sector also reflects the moderation in the growth of industrial production, which increased by only 3 percent in 1989 compared with an increase of 6 percent in 1988 .

The average revenue per kilowatthour of electricity sold during 1989 was 6.5 cents (Table 1). The highest average revenue per kilowatthour occurred in the residential sector at 7.6 cents (Table 26). The commercial and industrial sectors reported average revenue per kilowatthour of 7.2 and 4.7 cents, respectively, during 1989.

\section{Generation Growth is Modest}

The growth rate for total U.S. net generation reflected a similar slowdown, increasing by 3 percent in 1989 (Table 1) compared with a 5-percent increase in 1988 (Table 12). Nonetheless, a record level of generation was set when 2,784 billion kilowatthours of electricity were produced in 1989. The slower growth in the production of electricity was primarily due to milder temperatures and temperate economic conditions during 1989 in contrast to the relatively colder winter and hotter summer of the previous year.

Hydroelectric Generation Begins Recovery. Generation from hydroelectric plants during 1989 was 265 billion kilowatthours, 19 percent higher than in 1988 (Table 1). Although this marks a significant recovery of hydroelectric power, generation from this energy source in 1989 remained 6 percent below the average of the prior 10 years (1979 through 1988). Even though water conditions improved overall for the United States, some areas of drought persisted through 1989 (Figure 11). Conditions remained in the severeto-extreme drought category in central California and in the Central and North Central Regions of the country. Concurrently, the areas suffering from severeto-moderate drought conditions in 1989 were much smaller in comparison with 1988. Most of the East, as well as the South Central States, experienced normalto-moist conditions, representing an improvement from the scattered moderate-to-severe drought conditions during 1988. The combined-flow levels of the U.S. portions of the Mississippi, Columbia, and St. Lawrence Rivers were 33 percent higher during the water year, 1989, than the flow levels during the water year, 1988.

Nuclear Generation Sets Record. During 1989, generation of electricity from nuclear power achieved a record level of 529 billion kilowatthours; however, this was an increase of less than 1 percent over the prior year (Table 1). The average performance of nuclear units in 1989 was well above that for the last 10 years. Nonetheless, the annual increase in generation was the smallest since 1980 because of the exceptionally high performance of these units in 1988.

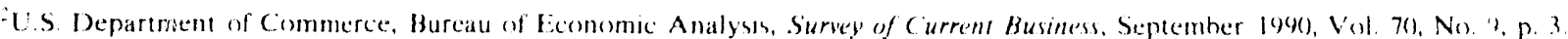

'Uniled States Department of the Interior, US Geological Survey, National W'aler Conditions, various issues (1989). The water year extends

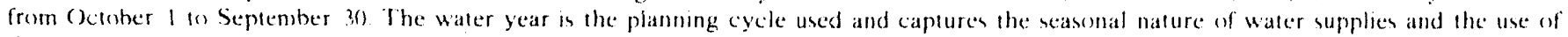
the waler.
} 


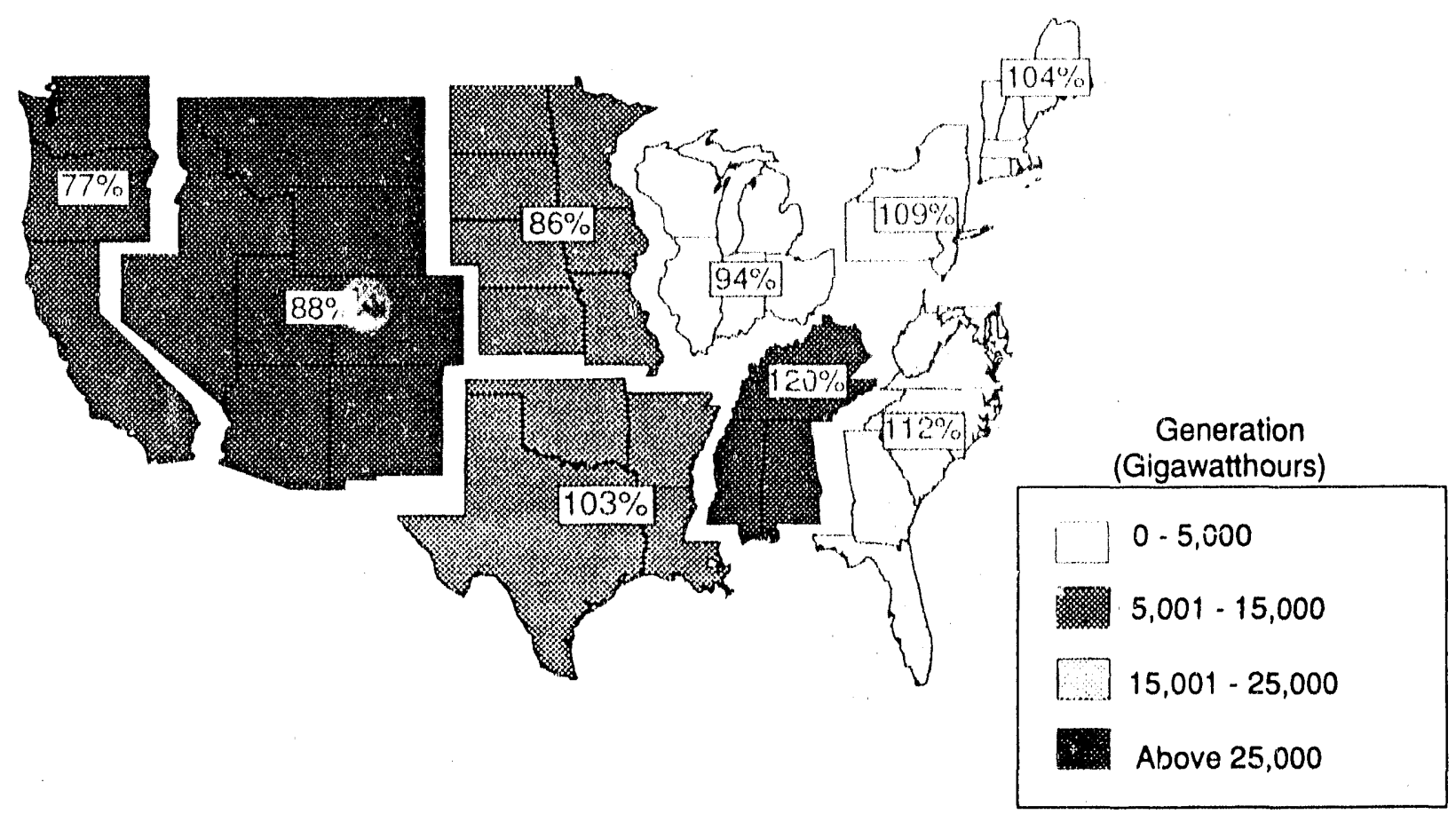

Note: Alaska and Hawaii are not included.

Sources: "Generation: Energy Information Administration, Form ElA-759, "Monthly Power Plant Report." -Precipitation: National Oceanic and Atmospheric Administration, 1989: Monthly State, Regional and National Heating Degree Days Weighted by Population (March 1989); Normal: State, Regional and National Monthly and Annual Precipitation Weighted by Area (August 1988).

In 1989, the average capacity factor for nuclear generating units was 62 percent; in 1988, it was 64 percent. ${ }^{4}$ Because of scheduled and unscheduled maintenance and/or refueling at several units, nuclear power declined in the first half of 1989 , but made a strong recovery in the second half. Contributing to the higher levels of nuclear-powered generation was the addition of three nuclear units during the year: Vogtle, Unit 2, operated by the Georgia Power Company; South Texas, Unit 2, operated by the Houston Lighting and Power Company; and Limerick, Unit 2, operated by the Philadelphia Electric Company. These units had a combined net summer capability rating of 3,391 megawatts.' During 1989, the 217-megawatt Fort St. Vrain nuclear Unit 1, owned by the Public Service Company of Colorado, was retired from service.
Coal Largest Contributor. Coal-fired generation continued to be the largest contributor to electric utility supply, providing 56 percent of total utility generation in 1989 , compared to 57 percent of total utility generation in 1988. Although a record was set when 1,554 billion kilowatthours of electricity were produced from coal-fired units in 1989, this level was only 1 percent higher than the level during the previous year (Table 12). Coal-fired generation during 1988 rose by 5 percent from the level of production in 1987. The slower rate of growth in electricity demand and a substantial recovery in hydroelectric generation contributed to the slower growth in coal-fired generation. Regionally, the highest level of generation from coal occurred in the East North Central Census Division, where 356 billion kilowatthours were produced in 1989 (Table 14). The South Atlantic Census Division had the second highest level, producing 332 billion

\footnotetext{
${ }^{4}$ Energy Information Administration, Monthly Energy Review. DOE/EIA-(X)35(9)/(07) (Washington, DC, October 19\%()), p. 85.

"Finergy Information Administration, Inventory of Power Plants in the United States, DOE/EIA-(X)95(89) (Washington, DC, September 1990), p. 3.
} 
kilowatthours. Together, these two Census divisions produced 44 percent of the total U.S. generation from coal in 1989.

Record Coal Cunsumption. In parallel with the increase in coal-fired generation, consumption of coal increased by 1 percent in 1989 (Table 1). The actual amount of coal consumed by electric utilities during 1989 was 767 million short tons, an all-time high, exceeding the record set in 1988. Electric utilities also increased their receipts of coal in 1989 to 753 million short tons of coal." This represented an increase of 4 percent $(25$ million short tons) from 1988 and established a record.

Coal Stocks Decline. The increased demand for coal can be attributed to changes in coal stocks at electric utilities over the last 2 years. Many electric utilities had prepared for a possible United Mine Workers (UMW) strike in late 1987 by increasing their stocks of coal; however, the strike was avoided. As a result, many electric utilities had excessively high coal stocks at the start of 1988. Throughout 1988, electric utilities reduced their stocks by limiting purchases of replacement coal. During 1989, electric utilities were more likely to purchase additional coal to replace coal consumed since the stock reduction was nearly complete. Consequently, receipts of coal increased at many electric utilities in 1989 compared with 1988. A very cold December also contributed to a significant drawdown in coal stockpiles. As of December 31, 1989, utilities reported coal stocks of 136 million short tons, a decrease of 7 percent from 1988 (Table 1), and the lowest endof-year level since 1978.

Cost of Coal Down. Another factor that may have contributed to increased use of coal in 1989 was its lower cost. The average delivered cost of coal received by electric utilities in 1989 was $\$ 30.15$ per short ton (Table 18), a decrease of $\$(0.49$ (2 percent) per short ton from that reported in 1988. The average delivered cost of coal purchased under contract was $\$ 30.38$ per short ton, a decrease of $\$ 0.48$ per short ton from the average reported in 1988 (Table 18). Coal purchased in the spot market averaged $\$ 29.07$ per short ton, tecreasing $\$ 0.23$ from 1988 levels. Many of the same factors responsible for a decrease in the cost of coal in 1988 contributed to the decline in 1989 costs. Among these factors were buy-outs and renegotiations of highpriced coal contracts, renegotiation of rail contracts for hauling coal, increased purchases of low-cost, spotmarket coal, and excess production capacity within the coal industry.

Petroleum- and (ias-Fired Generation Increase. During the first half of the year, petroleum-fired generation was 35 percent higher than it was during the corresponding period of 1988: however, petroleum-fired generation was 14 percent lower during the second half of the year than it was during the corresponding period of 1988 . Whereas, gas-fired generation during the first half of 1989 was slightly above the level during the first half of 1988 and 10 percent higher during the second half compared with the corresponding period in 1988. Nevertheless, generation from both gas- and petroleum-fired units, on an annual basis, increased in 1989. Gas-fired generation increased by 6 percent in 1989 to 267 billion kilowathours, while petroleumfired generation increased by 6 percent to 158 billion kilowatthours (Table 1).

Petroleum Costs Up, Receipts Down. Petroleum consumption in 1989 was at its highest level since 1981. During 1989, electric utilities consumed 267 million barrels of petroleum, 8 percent above the amount reported during the previous year (Table 1). Petroleum stocks as of December 31, 1989, totaled 61 million barrels, a decrease of 12 percent from 1988. This amount was also at its lowest end-of-year level since 1972. The shift from the use of petroleum to the use of gas during 1989 is indicative of changes in fuel costs. Petroleum costs during the year increased by 19 percent while the cost of gas increased by 4 percent. Gas and petroleum are substitute fuels in dual-fired units. The average cost of petroleum delivered to electric utilities was $\$ 18.17$ per barrel in 1989 , an increase from $\$ 15.33$ per barrel in 1988 ('Table 18). World-wide petroleum prices were generally higher in 1989 than in 1988. Record cold weather in December 1989 led to soaring demand for fuel oil and contributed to the increased cost of petroleum delivered to electric utilities during the year. Electric utilities reported receiving 24 million barrels of petroleum in December 1989, at an average cost of $\$ 22.43$ per barrel. Petroleum received by electric utilities in 1989 totaled 246 million barrels, an increase of 9 million barrels from 1988. The average cost of gas in 1989 was $\$ 2.42$ per thousand cubic feet delivered (Table 18). During the year, the average cost of gas delivered to electric utilities varied from a low of $\$ 2.30$ per thousand cubic feet in April to a high of $\$ 2.84$ per thousand cubic feet in December.

The Middle Atlantic, South Atlantic, and New England Census Divisions accounted for 86 percent (212 million barrels) of all petroleum receipts (Table 23). At the State level, New York ranked highest with 66 million barrels, followed by Florida and Massachusetts (40) and 29 million barrels, respectively). Receipts of petroleum to the South Allantic Census Division increased 9 million barrels from 1988 concurrent with a 13-percent increase in petroleum-fired generation. Receipts of petroleum to the Middle Atlantic Census Division increased by 3 million barrels in conjunction with an increase in consumption. Receipts to the New England Census Division, however, decreased by 1 million bar-

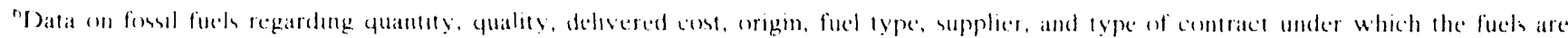

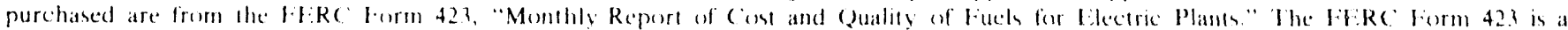

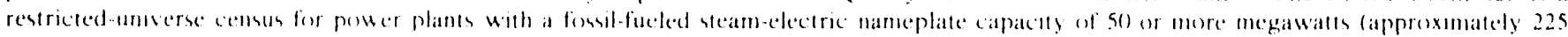
electric unliness.
} 
rels concurrent with lower consumption and generation levels for 1989. Receipts of petroleum to the Pacific Contiguous Census Division decreased by 4 million barrels, reflecting a drawdown in petroleum stocks.

Use of Gas Increases. Consumption of gas by electric utilities in 1989 increased o percent from that of 1988 (Table 1). The actual amount of gas consumed was 2,787 hillion cubic feet, compared 10 2,636 billion cubic feet consumed during 1988 . Receipts of gas at electric utilities in 1989 totaled 2,47.3 billion cubic feet, an increase of 110 billion cubic feet from 1988. Several clectric utilities in the New England, Middle Atlantic and South Atlantic Census Divisions reported increases in gas receipts due in part to the competitive price of gats versus petroleum. Combined, the West South Central and the Pacilic Contiguous Consus Divisions accounted for 1,860 billion cubic feet, 75 percent of total U.S. gas receipts (Table 23). Significant increases in gas receipts oceurred in the South Atlantic, Middle Allantic. Mountain and New England Census Divisions.

\section{Nuclear Dominates New Capability}

Nuclear capability dominaled new capability additions in 1989, accounting for 49 percent of the 7 gigawatts of capability in new units (Table 4). Coal-fired capability accounted for 28 percent of new unit additions. The remaining 2.3 percent of new additions was in gas turbine, internal combustion, hydroclectric, solar, and refuse-fucled steam units. In 1989, utilities retired less than 1 gigawatt of generating capability. As of the end of 1989, electric generating capability in the United States totaled 685 gigatwatts.

Electric utilities have also proposed adding 4 ! gigawatts of gensating capability in new units over the next 10) years (Table 10). (jas turbine, combined cycle, and coal-fired gemerating units account for 77 percent of these proposed capability additions.

\section{Rate of Fossil-Fuel Emissions Slows}

When fossil fuels are burned during the production of electricity, a variety of gases and particulates (including sulfur dioxide, nitrogen oxides, and carbon dioxide) are formed. If these substanc is are not captured by pollution control equipment, they are released into the atmosphere. (of the three major fossil fuels (coal, petroleum, and gas) used to generate electricity, coal has the highest average sulfur content. In addition, coal has historically provided the largest share (relative to other energy sources) of total U.S. generation. As a result of these two factors, generation from coal-fired power plants usually accounts for the largest share of sulfur dioxide emissions. Total fossil-fueled generation increased by 2 percent in 1989 from 1988; coal-fired generation rose by 1 percent in 1989 from 1988.

In 1989, the emissions of sulfur dioxide as a result of burning fossil fuels for the gencration of electricity were esimated to be 17 million short tons, less than 1 percent higher than the level in 1988 (Table 1). ${ }^{*} \mathrm{Ni}$ trogen oxide emissions in 1989 (7 million short tons) rose by slightly more than 1 percent from 1988. The level of carbon dioxide emissions in 1989 were 1,997 million short tons, an increase of 28 million short tons ( 1 percent) from 1988 . The smaller increase in emissions of sulfur dioxide, relative to the increase in generation, indicates the possibility that a larger perentage of the generation was produced using scrubbers. The U.S. average cost (including installation) for flue gas desulfurization units was $\$ 119$ per kilowatt in 1989 , about the same as the average installation cost in 1988 (Table 47). Average operation and maintenance costs for scrubbers declined each year from 1.61 mills per kilowatthour in 1986 to 1.34 mills per kilowatthour in 1989."

The average quality of fossil fuels in 1989 was relatively constant with that in 1488 (Table 46). The heat content in 1989 for coal was 10,428 Btu per pound; for petroleum, 149,260 Btu per gallon; and for gas, 1,030 Btu per cubic foot. The 1989 average sulfur content by weight) was 1.33 and 0.93 percent, respectively, for coal and petroleum. The average ash content for coal was 10 percent.

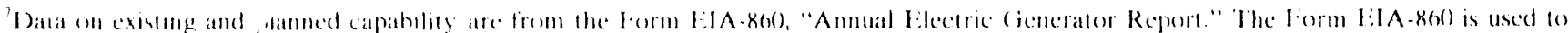

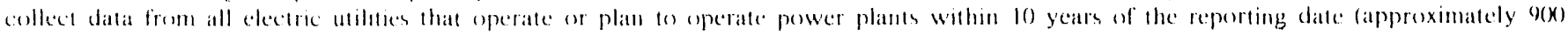
electric utilitices).

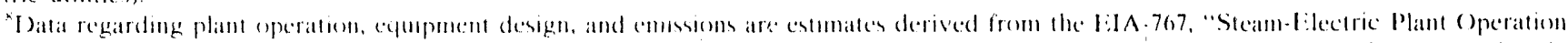

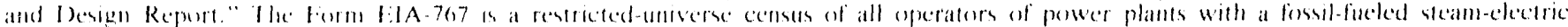

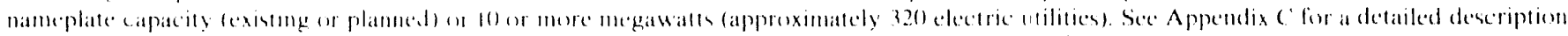

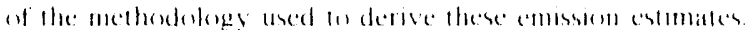

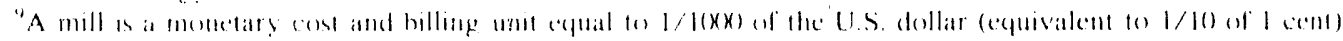




\section{Financial Growth Steady}

The U.S. electric utility industry in 1989 continued to experience steady growth in electric operating revenue. Financial performance was affected by aftertax write offs, accounting adjustments, and regulatory rate decisions. "For example, the Public Service Company of New Hampshire reported an aftertax write off of nearly $\$ 1$ billion in miscellaneous income deductions and extraordinary deductions. The majority of electric utilities in 1989 improved their financial position, but aggregate industry results were reduced by a relatively small number of financially troubled electric utilities.

In 1989, total electric operating revente at investorowned electric utilities (which provide 77 percent of sales of electricity to ultimate consumers) increased by nearly 5 percent reaching $\$ 151$ billion and net income increased by 8 percent to a total of $\$ 17$ billion (Table 31). Electric operating expenses increased from $\$ 115$ billion to $\$ 122$ billion ( 5 percent). These increases in nonfuel operation and maintenance expenses, despite a nominal and real reduction in nuclear operation and maintenance expenses in 1989, impacted negatively on the earnings of the electric utility industry. Cost disallowances and extraordinary losses of nearly $\$ 4$ billion impacted earnings significantly, although less than the impact experienced in 1988. Because of the continuing trend toward cost disallowances and extraordinary losses, the electric utility industry has deferred large capital investments in new large central station power plants, causing further deterioration and a slowing in future earnings. Construction work in progress declined over 16 percent in 1989, marking the sixth consecutive year of declining capital investments. However, total assets increased by 3 percent and accumulated depreciation grew by 10 percent effecting completion of construction programs. This increase in toial assets was accompanied by an associated increase in assets relating to electric utility plant, which rose by 5 percent in 1989 versus an increase in 1988 of 7 percent.

Due to the different fiscal years reported by publicly owned electric utilities, data for only those utilities whose fiscal year ended June 30, 1989, and December 31, 1989, are provided in this report. For publicly owned electric utilities with a fiscal year ending June 30,1989 , electric utility operating revenue was $\$ 10$ billion; electric operating expenses were $\$ 8$ billion (Table $36)$; and assets relating to electric utility plant were $\$ 25$ billion (Table 37). For publicly owned electric utilities with a fiscal year ending December 31, 1989 , electric utility operating revenue was $\$ 10$ billion; electric operating expenses were $\$ 9$ billion; and assets relating to electric utility plant were $\$ 35$ billion. The net income of publicly owned electric utilities with a fiscal year ending December 31, 1989 (\$-317 million), was impacted by the Sacramento Municipal Utility District, which reported extraordinary deductions of nearly $\$ 60()$ million This deduction reflects the abandonment of the Rancho Seco Nuclear Plant and the subsequent write off at the year end.

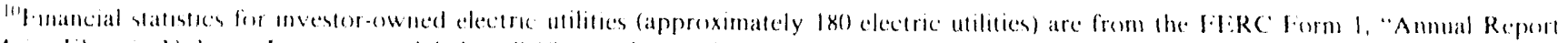

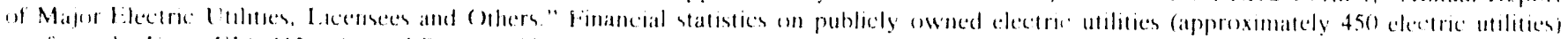

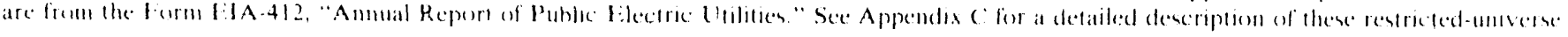
censtise:s
} 
Figure 12. Sales to Uitimate Consumers by Sector, 1989

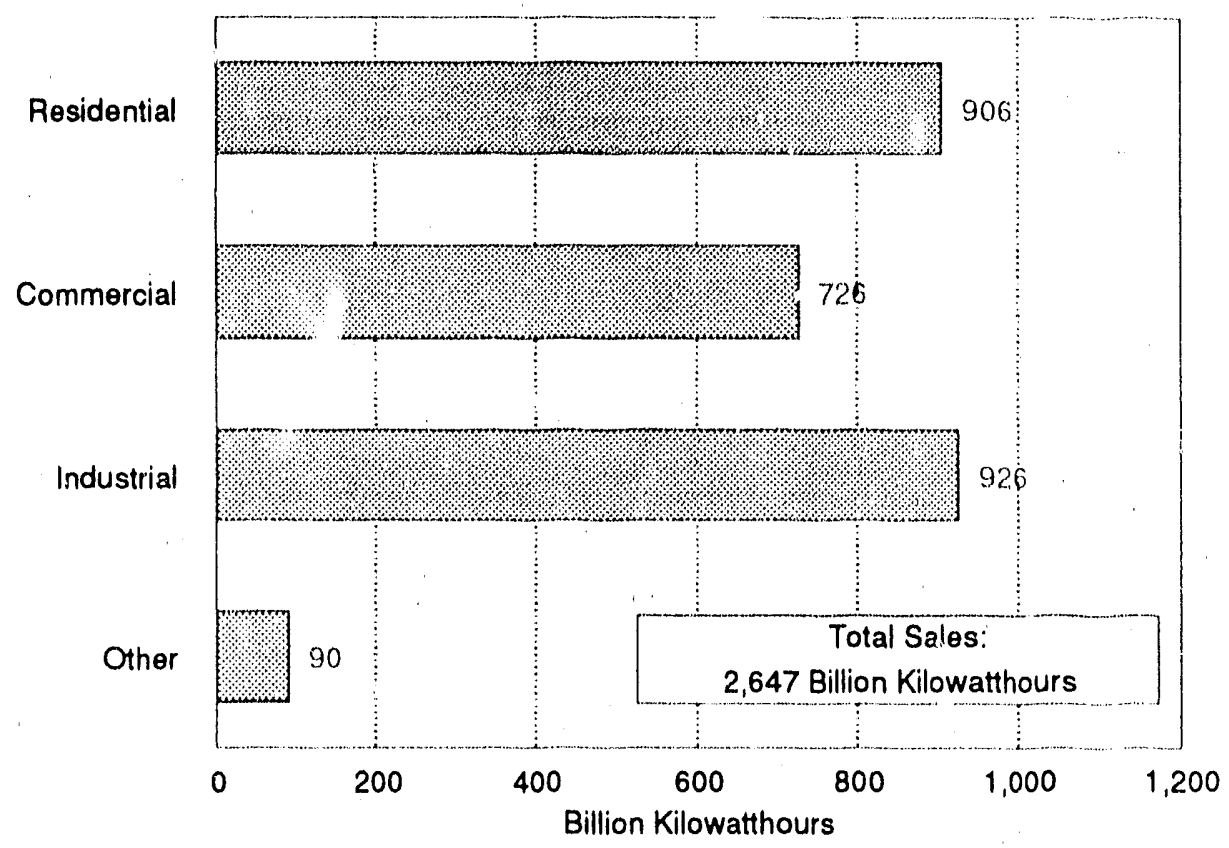

Notes: Other includes public street and highway lighting, other sales to public authorities, sales to railroads and raiiways, and interdepartmental sales. Totals may not equal sum of components because of independent rounding.

Source: Energy Information Administration, Form ElA-861, "Annual Electric Utility Report."

Figure 13. Share of Sales to Ultimate Consumers by Sector, 1989

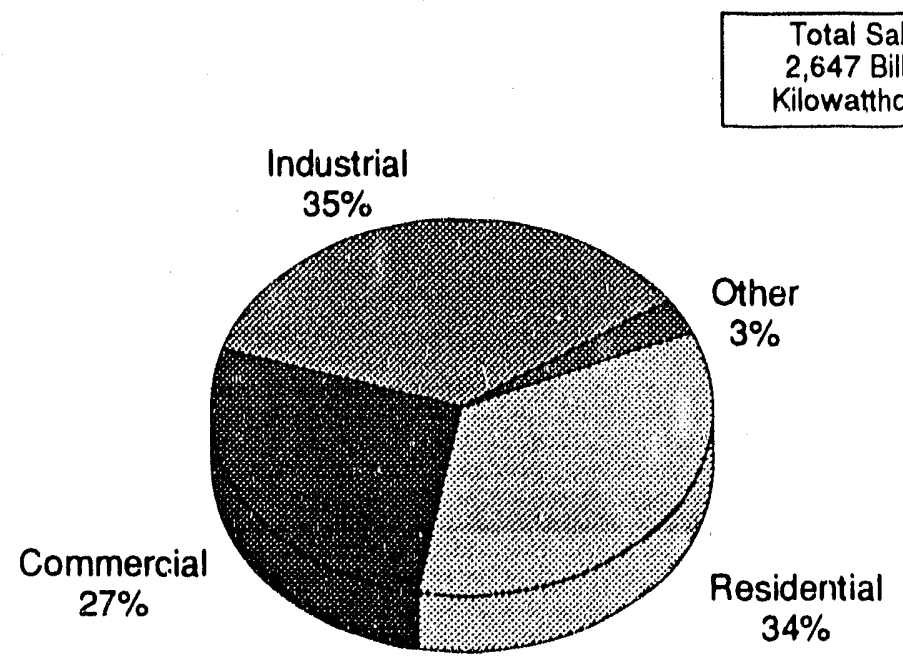

Notes: Other includes public street and highway lighting, other sales to public authorities, sales to railroads and railways, and interdepartmental sales. Totals may not equal sum of components because of independent rounding.

Source: Energy Information Administration, Form ElA-861, "Annual Electric Utility Report." 
Figure 14. Average Revenue per Kilowatthour by Sector, 1989

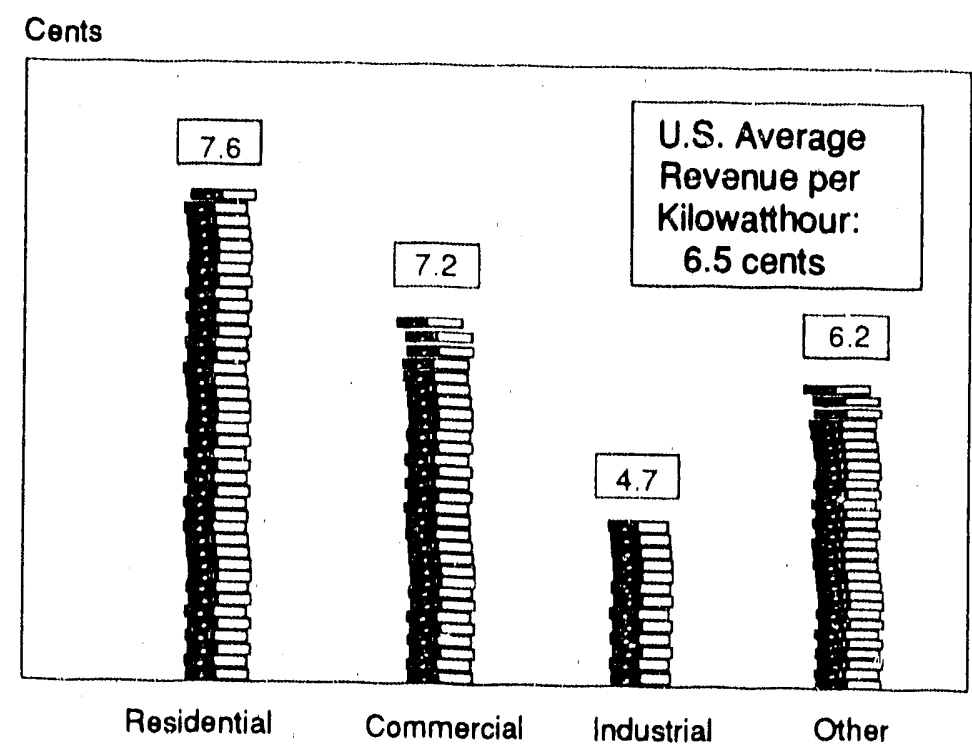

Note: Other includes sales to public street and highway lighting, other sales to public authorities, sales to railroads and railways, and interdepartmental sales.

Source: Energy Information Administration, Form ElA-861, "Annual Electric Utility Report."

Figure 15. Net Generation by Energy Source, 1989

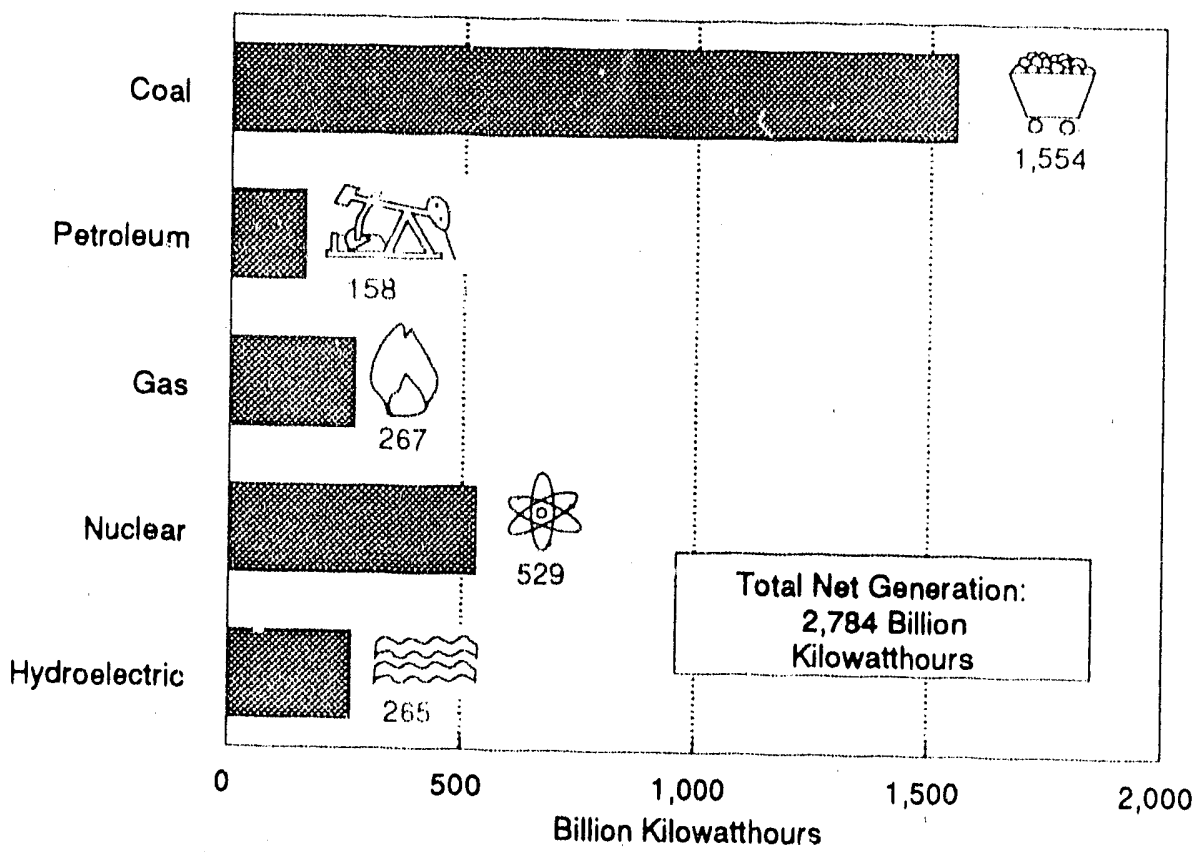

Notes: Total net generation value includes 11 billion kilowatthours of generation by other energy sc'rrces including geothermal, wood, wind, waste, and solar. Totals may not equal sum of components because of
independent rounding.

Source: Energy Information Administration, Form ElA-759, "Monthly Power Plant Report." 
Figure 16. Share of Net Generation by Energy Source, 1989

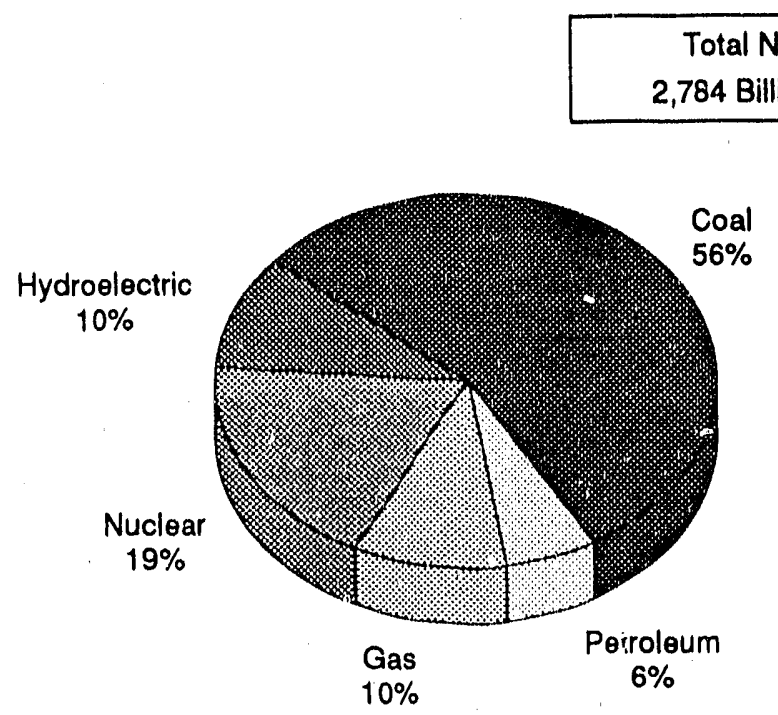

Notes: Total value includes generation from other energy sources (geothermal, wood, wind, waste, and solar), which represents less than 1 percent of total generation. Totals may not equal sum of components because of independent rounding.

Source: Energy Informiation Administration, Form ElA-759, "Monthly Power Plant Report."

Figure 17. Monthly Net Generation, 1989 and 1988

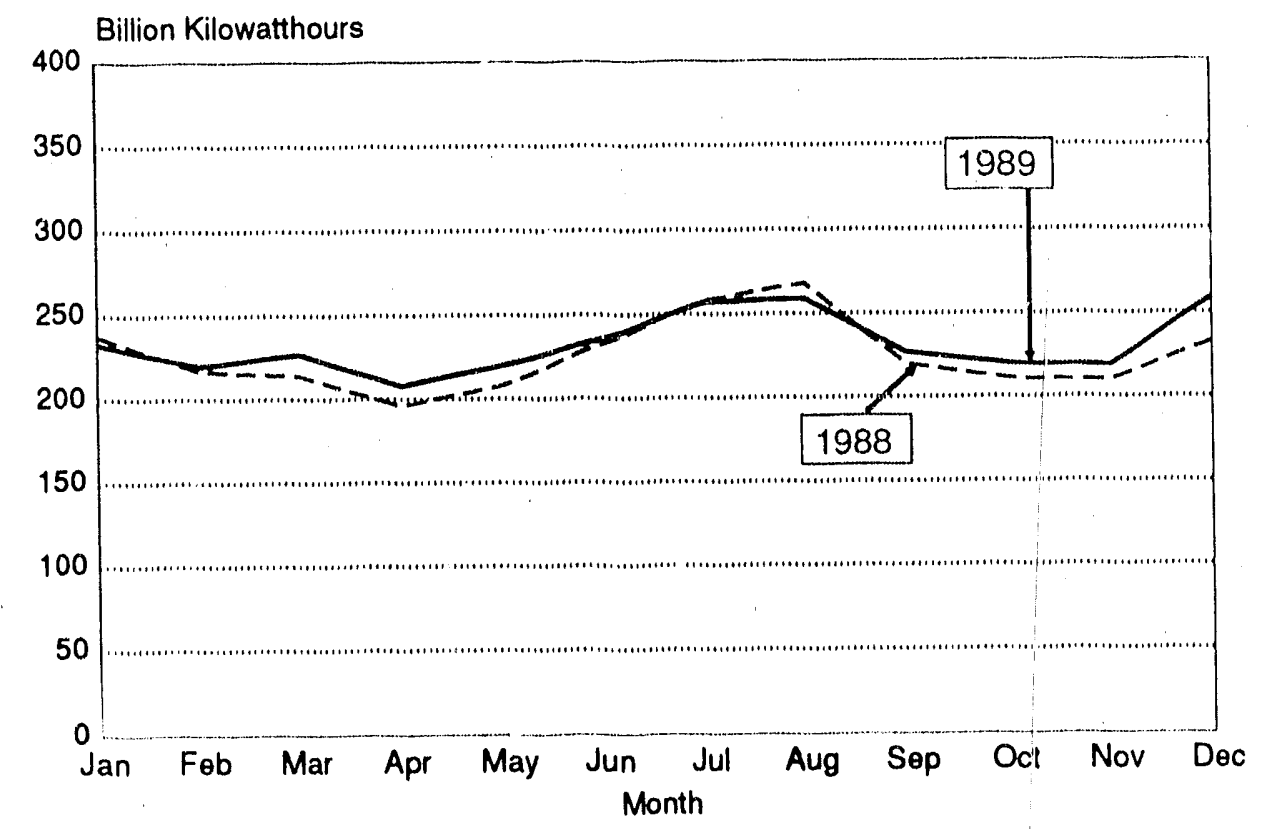

Source: Energy Information Administration, Form ElA-759, "Monthly Power Plant Report." 
Figure 18. Generating Capability by Energy Source, 1989

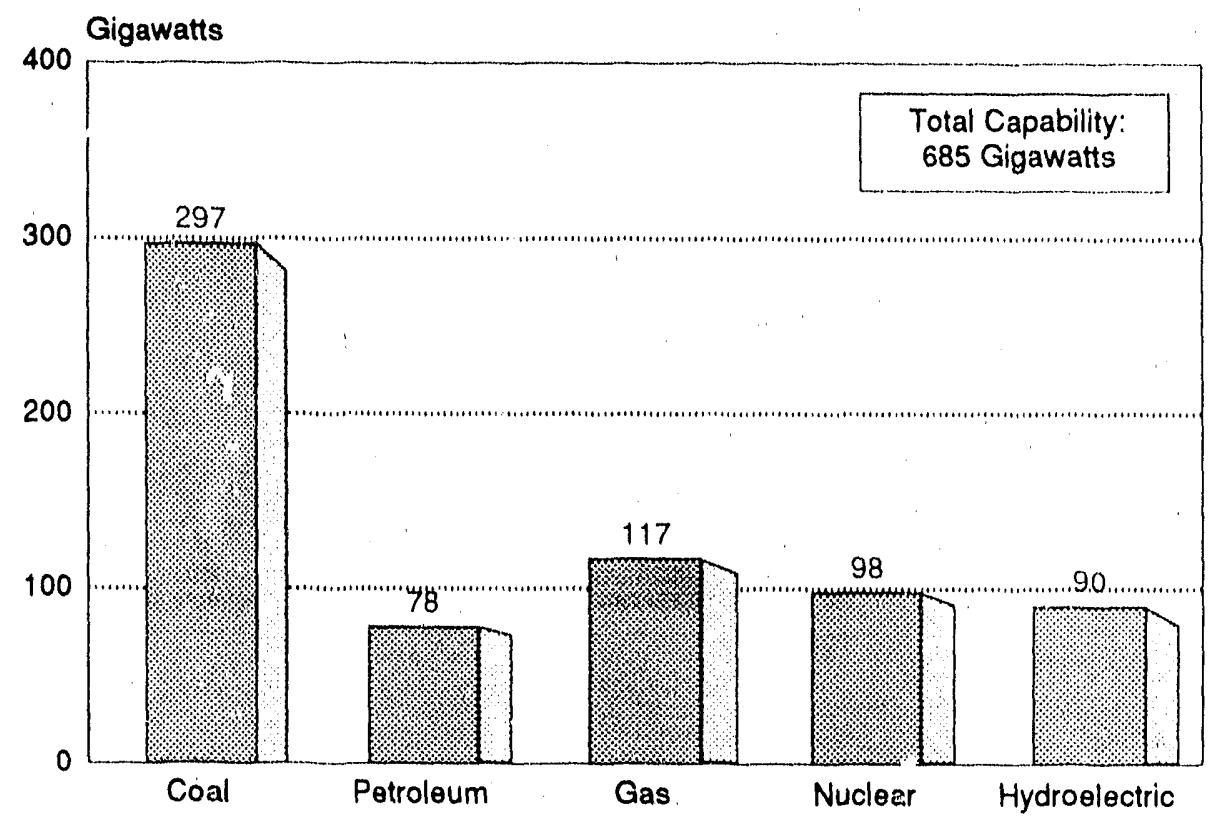

Notes: Total capability value includes 4 gigawatis of other generating capability (geothermal, refuse, waste heat, waste steam, solar, wind, and wood). Totals may not equal sum of components because of independent rounding.

Source: Energy Information Administration, Form ElA-860, "Annual Electric Generator Report."

Figure 19. Share of Generating Capability by Energy Source, 1989

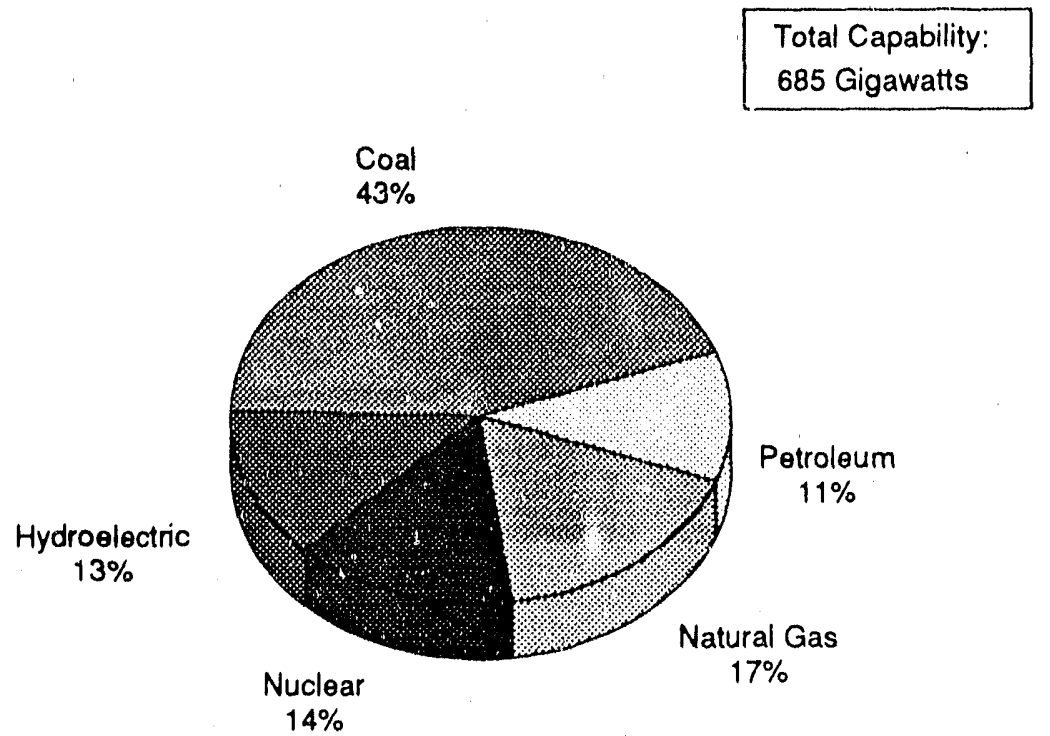

Notes: Total capability value includes other generating capability (geothermal, refuse, waste heat, waste steam, solar, wind, and wood), which represents 1 percent of total capability. Totals may not equal sum of components because of independent rounding.

Source: Energy Information Administration, Form ElA-860, "Annual Electric Generator Report." 
Table 1. Electric Power Summary Statistics for the United States, 1989 and 1988

\begin{tabular}{|c|c|c|c|}
\hline Item & 1989 & 1988 & $\begin{array}{c}\text { Percent Change from } \\
\text { Prevlous Year }\end{array}$ \\
\hline Generating Capability (glgawatts)' $\ldots . .$. & 685 & 678 & 1.0 \\
\hline 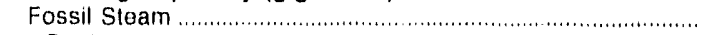 & 442 & 440 & 5 \\
\hline Coal & 297 & 295 & .7 \\
\hline 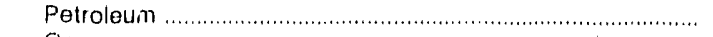 & 51 & 51 & 4 \\
\hline 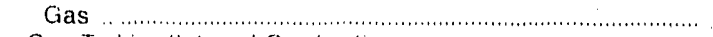 & 94 & 94 & -.1 \\
\hline 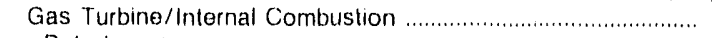 & 50 & 49 & 3.0 \\
\hline Petroleum : & 27 & 26 & 2.5 \\
\hline 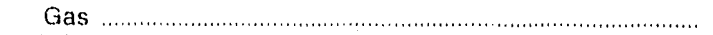 & 23 & 22 & 3.7 \\
\hline 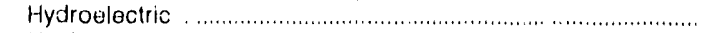 & 90 & 90 & 2 \\
\hline 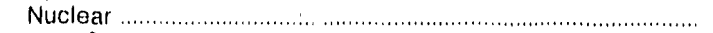 & 98 & 95 & 3.7 \\
\hline Other $^{2}$ & 4 & 4 & -5.0 \\
\hline Generation (billion kilowatthours) & 2,784 & $2,7 n 4$ & 3.0 \\
\hline Coal . & 1,554 & 1,541 & 8 \\
\hline 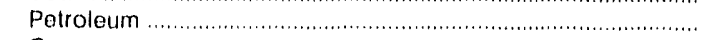 & 158 & 149 & 6.3 \\
\hline 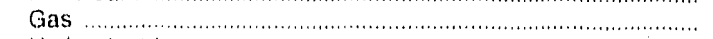 & 267 & 253 & 5.5 \\
\hline Hydroelectric & 265 & 223 & 18.9 \\
\hline 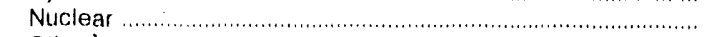 & 529 & 527 & .5 \\
\hline Other' ${ }^{3}$ & 11 & 12 & -5.6 \\
\hline \multicolumn{4}{|l|}{ Consumptlon } \\
\hline Coal (million short tons) & 767 & 758 & 1.1 \\
\hline 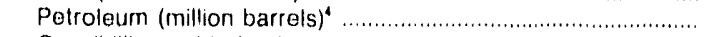 & 267 & 248 & 7.8 \\
\hline Gas (billion cubic feet) & 2,787 & 2,636 & 5.7 \\
\hline \multicolumn{4}{|l|}{ Stocks } \\
\hline 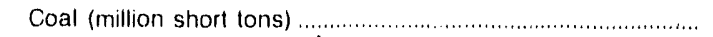 & 136 & 147 & -7.3 \\
\hline 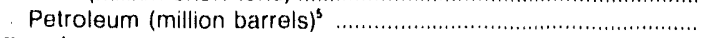 & 61 & 69 & -11.6 \\
\hline Recelpts & & & \\
\hline . & 753 & 728 & 3.5 \\
\hline 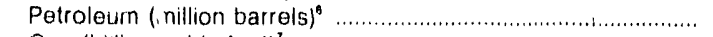 & 246 & 237 & 4.0 \\
\hline 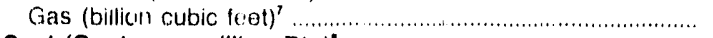 & 2,473 & 2,363 & 4.6 \\
\hline \multicolumn{4}{|l|}{ Cost (Cents per million Btu) } \\
\hline Coal & 144.6 & 146.6 & -1.4 \\
\hline 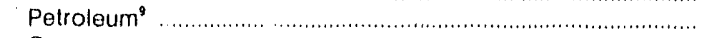 & 289.3 & 243.9 & 18.6 \\
\hline 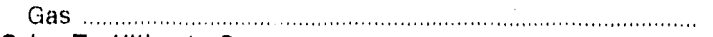 & 235.5 & 226.3 & 4.0 \\
\hline \multicolumn{4}{|l|}{ Sales To Ultimate Consumers } \\
\hline (billion kllowatthours) & 2,647 & 2,578 & 2.7 \\
\hline 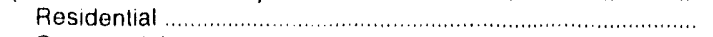 & 906 & 893 & 1.4 \\
\hline 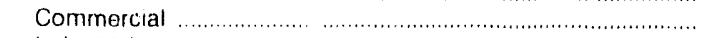 & 726 & 699 & 3.8 \\
\hline 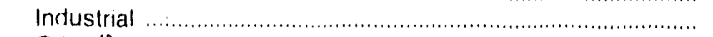 & 926 & 896 & 3.3 \\
\hline Other ${ }^{10}$ & 90 & 90 & .2 \\
\hline \multicolumn{4}{|l|}{ Revenue From Sales to Ultimate Consumers } \\
\hline (billion dollars) & 171 & 164 & 4.3 \\
\hline 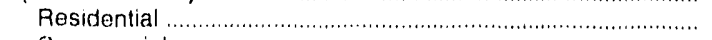 & 69 & 67 & 3.7 \\
\hline 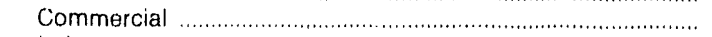 & 52 & 49 & 6.1 \\
\hline 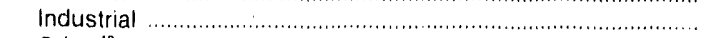 & 44 & 42 & 3.7 \\
\hline Other ${ }^{10}$ & 6 & 6 & 1.1 \\
\hline Average Revenue per Kllowatthour (cents) & 6.5 & 6.4 & 1.6 \\
\hline 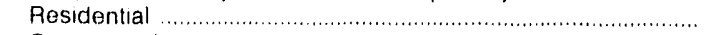 & 7.6 & 7.5 & 2.2 \\
\hline 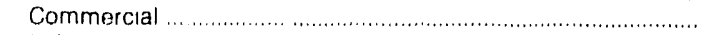 & 7.2 & 7.0 & 2.2 \\
\hline 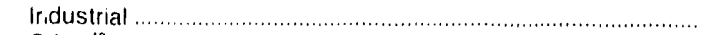 & 4.7 & 4.7 & .5 \\
\hline 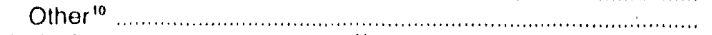 & 6.2 & 6.2 & .9 \\
\hline \multicolumn{4}{|l|}{ Emissions (million short tons)" } \\
\hline 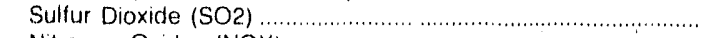 & 17 & 17 & .3 \\
\hline Nitrogen Oxides $(\mathrm{NOX})$ & 7 & 7 & 1.3 \\
\hline Carbon Dioxide $(\mathrm{CO} 2)$ & 1,997 & 1,969 & 1.4 \\
\hline
\end{tabular}

Net surnmer capability based on primary enerqy source.

2 Includes geothermal, refuse, waste heat, wa: to steam, solar, wind, and wood

Includes geothermal, wood, wind, waste, and solar.

- Does not include petroleum coke. Petroleum coke consumption in 1989 was 517 thousand short tons and in 1988 was 409 thousand short tons.

5 Does nol include petroleum coke. Petroleum coke stocks at the end of 1989 were 105 thousand short tons and in 1988 were 86 thousand short tons.

Does not include petroleum coke. Petroleum coke receipts in 1989 were 495 thousand short tons and in 1988 were 356 thousand short tons.

Includes small amounts of coke-oven, refinery, and blast-furnace gas.

- Average cost of fuel delivered to plants with a fossil-fueled steam-electric nameplate capacity of 50 or more megawalts; avarage cost values are weighted.

- Does not include petroleum coke. Petroleum coke cost in 1989 was 85.7 cents per million Btu and in 1988 was 97.2 cents per million Btu.

10 Includes public street and highway lighting, other sales to public authorities, sales to railroads and railways, and interdepartmental sales.

1 Includes only those power plants with a fossil-fueled steam-electric nameplate capacity (existing or planned) of 10 or more megawatts.

Notes: -Data on capability, generation, consumption, stocks, receipts and costs of fossil fuels for 1989 are final; other 1989 data are preliminary

- Totals may not equal sum of components because of independent rounding. Percent change is calculated before rounding.

Suurces: -Energy Information Administration, Form ElA-759, "Monthly Power Plant Feport"; Form ElA-860, "Annual Electric Generator Report"; Form

ElA-861, "Annual Electric Utility Report"; Form ElA-767, "Steam-Electric Plant Operation and Design Report." •Federal Energy Regulatory Commission

FERC Form 423, "Monthly Report of Cost and Quality of Fuels for Electric Plants." 


\section{Outlook Through 2010}

\section{Clean Air Act}

On November 15, 1990, President Bush signed the Clean Air Act Amendments of 1990 into law. These projections are based, however, on legislation in effect is of June 1990, and do not include subsequent legislation.

Many decisions concerning the supply of electricity will confront the electric utility industry during the 1990's and beyond. At present, the industry is under considerable pressure to modify its traditional approach to satisfying consumer requirements. For example, regulatory commissions are encouraging utilityinitiated programs to lower energy consumption and thereby limit growth in demand. This section of the Electric Power Annual provides projections for the demand for electricity, the price of electricity, the supply if electricity, and fucl use by electric utilities through 2010.11

\section{Major Assumptions}

Macroeconomic growth is a major determinant of growth in the demand for electricity, that in turn affects capacity planning, fuel use, and price. These projections are based on the assumption that from 1989 through 2010, the real gross national product (GNP) grows at an average annual rate of 2.4 percerit. In addition, weather conditions are assumed to be normal. The projections presented in this section are made, assuming that existing laws and regulations remain in effect through 2010.

\section{Electricity's Share of Demand Increases}

The assumption of moderate economic growth coupled with expected normal weather results in moderate growth in the demand for electricity. Sales of electricity are expected to grow at an average rate of 2.3 percent per year through 2010 (Table 2). Electricity demand is expected to grow faster than the demand for other energy sources during the forceast period because real electiocicy prices remain steady while those of primary fuels increase. Of the increase in primary energy consumption from 1989 through 2010, 66 percent is accounted for by electricity. During the period, electricity will account for 44 percent of the increase in end-use energy consumption. Because of the construction of new, more electricity-intensive buildings, the commercial sector (exclusive of street lighting and transportation) is projected to show the strongest rate of growth in electricity consumption (3.2 percent per year). An expected increase in the production of equipment and machinery leads to a 2.5 -percent annual growth per year in the consumption of electricity in the industrial sector. The residential sector will experience the slowest rate of growth in electricity consumption (just under 1.9 percent per year) because of a slowdown in new home construction, as well as, thermal efficiency improvements in existing structures.

\section{Real Price Remains Stable}

Real electricity prices (in 1989 cents per kilowatthour) are expected to remain relatively stable from 1989 through 20!0. Prices experience a small decline before 2000 then rise slightly by 2010 , reaching 7.1 cents per kilowatthour. ${ }^{12}$

Although real electricity prices increase slightly during the forecast period, the relative proportions of two of the price components (fuel and capital) reflect significant shifts. Capital cost reduction. offset increased fuel costs $\mathrm{d}$ ing the next 10 years. Following the turn of the century, however, capital costs (per kilowathour) level off. Therefore, increases in fuel costs result in increases in the price of electlicity. The third component of electricity prices (operation and maintenance costs) remains fairly stable through 2010.

\section{Resources Are Diverse}

To meet the expected growth in demand, a variety of resources will be used. In the near term, electric utilities will utilize existing capacity more. For example, the average capacity factor for a coal-fired plant will increase from 60 percent in 1989 to 68 percent in 2010. In addition, some utilities will refurbish many of their older existing plants; 346 gigawatts of fossil-steam capacity are assumed to be life extended by 2010 with 51 gigawatts to be retired. Furthermore, electric utilities are expected to purchase 33.3 billion kilowathours of electricity from nonutility producers.

\footnotetext{
"The discussion in this section is a summary of the base case projections provided in the Fanergy Information Adminustration, annual Oullosk for U.S. Electric Power 1990), DOE:/EIA-1)474(90)(Washington, DC, January 1990).

"The projected prices, in 1989 cents per kilowathour, are from model simulations and represent average revenue per kilowatthour of demand over all consumer classes.
} 
At the time of these forecasts, electric utilities had reported plans for building 39 gigawatts of capacity by 2010. However, since utilities are only required to submit plans for capacity expansion for the next 10 years, an additional 232 gigawatts of unplanned, unannounced, or unreported additions will be needed by 2010 to meet demand.

Net imports of electricity are expected to achieve 68 billion kilowathours by 2010 . While this represents a small share of total electricity demand, imports of electricity have important impacts regionally (particularly in the New England area, where imported power will supply up to 17 percent of dremand during the forecast period.)

\section{Gas-Fired Generation Share Increases}

Gas-fired generation more than doubles by 2000 , accounting for 17 percent of U.S. generation, but declines to 14 percent by 2010 . Gas will surpass nuclear power to become the second-largest contributor to electric utility generation by 2000 . Use of petroleum will also increase through 2000 , then decline by 2010 as use of other energy sources increases. Coal will retain the majority share of U.S. generation --declining somewhat from its 56-percent share in 1989 to 52 percent in 200)( - but then attaining 60 percent of total U.S. generation by 2010 .

\section{Uncertainty of Projections}

These projections do not provide unqualified predictions for the future since a model cannot address the complexities of the real world. These projections represent expectations under a given set of assumptions; should these conditions change in any way, the projections would be affected accordingly. The uncertainty inherent in these projections should be recognized in order that the projections can be used in the proper context.

\section{Sources}

The base case projections provided in this section are summarized from the Energy Information Administration, Annual Outlook for U.S. Electric Power 1990 (AOEP). The AOEP contains national level projections of sources and uses of electricity through $2010 \mathrm{in}$ the body of the report, as well as detailed assumptions and regional level projections in the appendices. The projections in the AOEP were made using the Intermediate Future Forecasting System and energy demands were obtained from the PC-AEO forecasting model (see AOEP, Appendix A, for a detailed discussion of these models and their assumptions). The results shown in both this report and the AOEP are consistent with those provided in the Annual Energy Outlook $1990^{13}$

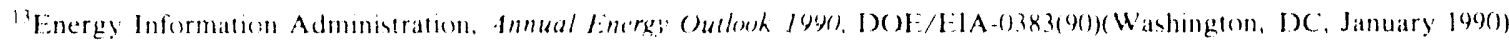


Table 2. Electris Power Projections for the United States Through 2010

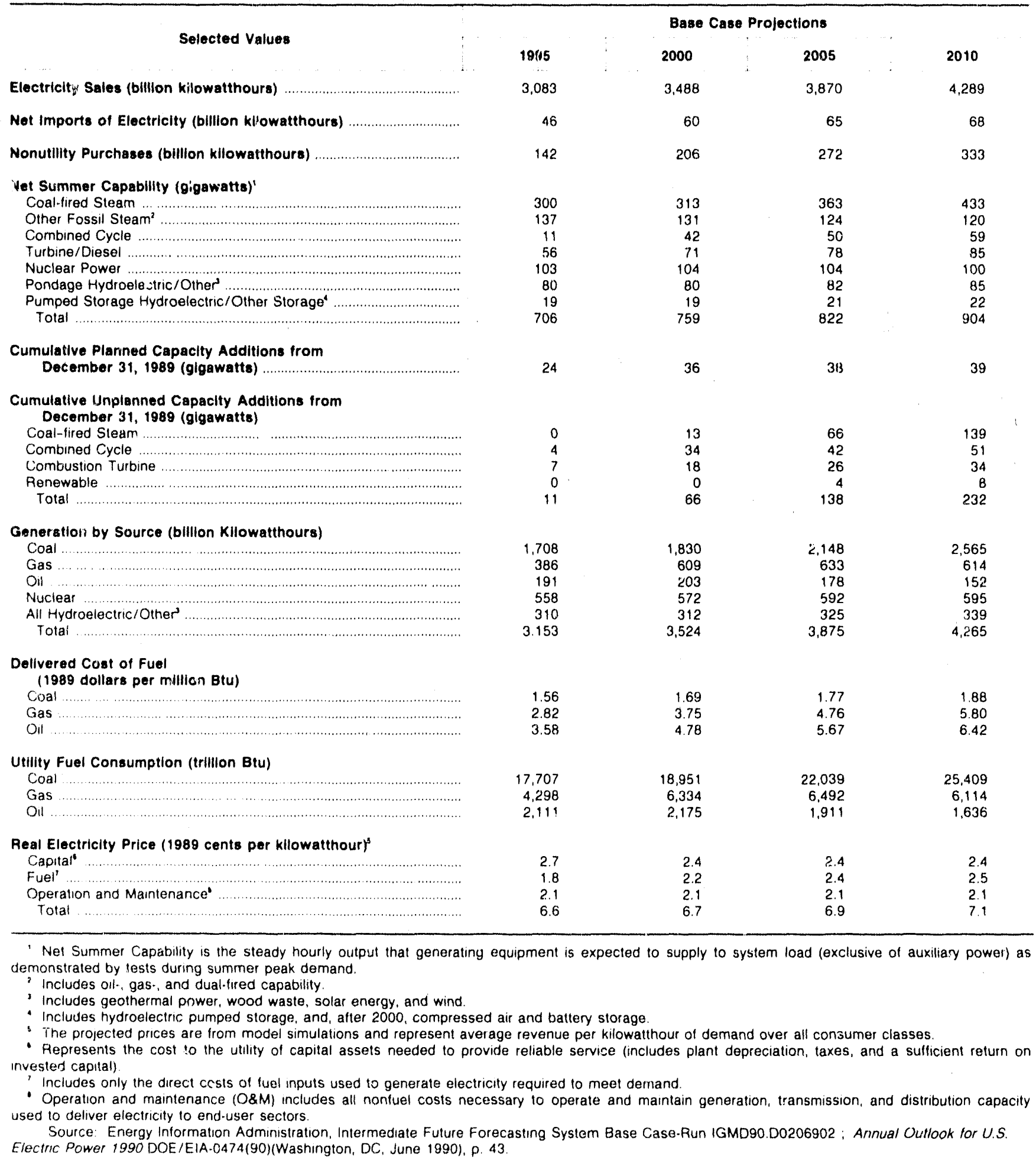

On November 15, 1990, President Bush signed the Clean Air Act Ammendments of 1990 into law. These projections are based. however, on legislation in effect as of June 1990, and do non include subsequent legislation. 


\section{Generating Capability}

Mo, re than one-third of the consumption of primary energy in the Nation is used to generate electricity. ${ }^{14}$ Consumers expect electricity to be instantly available, that is, at the flick of a switch. In fact, electricity is so important to the functioning of our society that its unavailability is newsworthy. Since the Northeast blackout of 1965, the electric power industry has organized to ensure that an adequate supply of electricity is available to meet all demand requirements at any given instant, both now and in the future. In order to meet these requirements, the electric power industry has built and maintained capacity to produce electricity for various types of demand and load requirements. This chapter provides an inventory of the capability of various methods for converting energy into electricity, as well as, providing information regarding the plans the industry has for building additional capacity.

\section{Background}

Although energy cannot be created or destroyed, it can be converted from one form into another form. Electricity is the result of a conversion process that is induced by rotating a magnet within a system of coiled wires, thus converting mechanical energy into electrical energy. The machine that performs this type of energy conversion is an electric generator. The device that causes the magnet to spir is a prime mover. A generating unit includes the electrical generator and all associated or physically connected equipment (such as a prime mover) that are operated together to produce electricicy.

The generating units operated by an electric utility vary by intended usage, that is, by the type of load requirements the utility must meet. The load requirements placed on an electric utility are generally categorized as base load, intermediate load, and peak load. A baselo. I generating unit is normally used to satisfy all or part of the minimum load of the system and, as a consequence, produces electricity at an essentially constant rate and runs continuously. These units are generally the newest, largest, and most efficient of the three types. (The operating efficiency of a generating unit is a function of the amount of net heat that it can extract from the energy source for use in the production of electricity.) A peakload generating unit, which is normally the least efficient of the three types of units, is used to meet requirements during the periods of greatest load on the system. Intermediate-load generating units meet system requirements that are greater than baseload but less than peak load. These units are used during the transition between baseload and peakload requirements. Utilities also have reserve or standby generating units, which are available to the system in the event of an unexpected increase in load or an unexpected outage within the system. Consequently, an inventory of net capability must account for reserve or standby capability, as well as generating units that are not available to the system for various reasons (such as routine maintenance).

Net capability in this report, unless otherwise stated, refers to that which is operable. Operable capability includes both active and inactive capability. Once a new generator has been declared available to generate power to the electrical grid, it is considered a part of the operable capability of the utility nill it is retired from service. Generating units that are used for standby service, generators that are placed in cold reserve or cold standby, and generators that are out of service for an extended period (exceeding one year) comprise the inactive operable capability. As of year-end 1989, about 4 percent of the operable capability was inactive. ${ }^{19}$ The active operable capability includes the capability of generators that are generating or a'vailable to generate. However, this active operable capability does not preclude generators that may be down for scheduled maintenance, refueling, or forced outages.

An electric utility plant (station) contains generating units and auxiliary equipment that are used to convert various types of energy into electric energy. A fossilfueled generating unit may be designed to use (burn) one or more fossil fuels to produce electricity. in generating unit capable of burning more than one fossil fuel is referred to as a dual-fired unit. ${ }^{16}$ Some dual-fired units can only burn one fue.' at a time (that is, the fuels

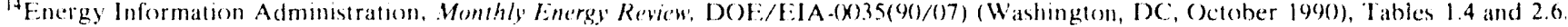
p. 31

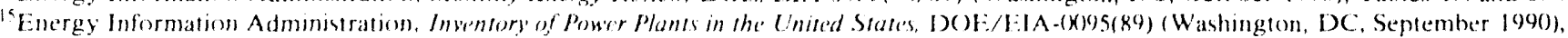

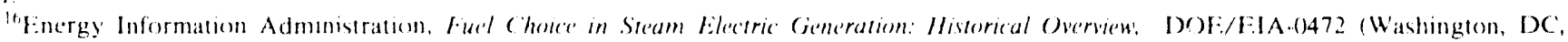
August 1985).
} 
are fired sequentially), while others can burn more than one fuel simultaneously (concurrent firing of different fuels). A sequentially fired unit generally uses one fossil fuel as its primary energy source but can switch to a second fossil fuel as an alternate energy source. Unless stated otherwise, information regarding generating capability in thi, report is based on the primary energy source.

\section{Prime Movers}

Electric utilities use a variety of prime movers based on the loads, availability of fuels, and energy requirements of the utility. The most common prime movers are the steam turbine, internal-combustion engine, gas combustion turbine, water turbine, and wind turbine. Most prime movers used to produce electricity today are turbines (a series of blades mounted on a shaft against which fluids are forced thus rotating the shaft connected to the generator). A turbine converts the kinetic energy of the moving fluid (liquid or gas) to mechanical energy. The fluids most commonly used in turbines are steam, hot air or combustion products, and water. The energy sources most often used with prime movers are the fossil fuels -- coal, petroleum, and natural gas.

Steam-Turbine Generating Units. Most of the electricity in the United States is produced in steam turbines. In a fossil-fueled steam turbine, the fuel is burned in a boiler to produce steam. The resulting steam then turns the turbine blades that turn the shaft of the generator to produce electricity. In a nuclear-powered steam turbine, the boiler is replaced by a reactor containing a core of nuclear fuel (primarily enriched uranium). Heat produced in the reactor by fission of the uranium is used to make steam. The steam is then passed through the turbine generator to produce electricity, as in the fossil-fueled steam turbine. Steam-turbine generating units are used primarily to serve the base load of electric utilities. Fossil-fueled steam-turbine generating units range in size (nameplate capacity) from 1 megawatt to over 1,000 negawatts. Nuclear-powered steamturbine generating units in operation today range in size from 75 megawatts to over 1,400 megawatts.

Gas Turbine Generating Units. In a gas turbine (combustion-turbine) unit, hot gases produced from the combustion of natural gas and distillate oil in a high-pressure combustion chamber are passed directly through the turbine, which spins the generator to produce electricity. Gas turbines are commonly used to serve the peak loads of the electric utility. Gas turbine units are generally less than 100 megawatts in size. Their wide choice of site locations and quick start-up time compared to steam-turbine generating units make them suitable for peaking, emergency, and reserve power.

The gas turbine, as is typical with peaking units, has a lower efficiency than the steam turbine used for baseload power. The efficiency of the gas turbine is increased when the gas turbine is coupled with a steam turbine in a combined cycle operation. In this system, the hot gases, which have already been used to spin one turbine generator are moved to a waste-heat recovery steam boiler where the water is heated to produce steam that in turn produces electricity by running a second steam-turbine generator. In this way, two generators produce electricity from one initial fuel input. All or part of the heat required to produce steam may come from the exhaust of the gas turbine. Thus the steam-turbine generator may be supplementarily fired in addition to the waste heat. Combined-cycle generating units generally serve intermediate loads.

Internal-Combustion Engines. These prime movers have one or more cylinders in which the combustion of fuel takes place. The engine, which is connected to the shaft of the generator, provides the mechanical energy to drive the generator to produce electricity. Internal-combustion (or diesel) generators can be easily transported, can be installed upon short notice, ind can begin producing electricity nearly at the moment they start. Thus, like gas turbines they are usually operated during periods of high demand for electricity. They are generally about 5 megawatts in size.

Hydroelectric Generating Units. Hydroelectric power is the result of a process in which flowing water is used to spin a turbine connected to a generator. The two basic types of hydroelectric systems are those based on falling water and those based on natural river current. In the first system, water accumulates in reservoirs created by the use of dams. This water then falls through conduits (penstocks) and applies pressure against the turbine blades to drive the generator to produce electricity. In the second system, called a runof-the-river system, the force of the river current (rather than falling water) applies pressure to the turbine blades to produce electricity. Since run-of-the-river systems do not usually have reservoirs and cannot store substantial quantities of water, power production from this type of system depends on seasonal changes and stream flow. These conventional hydroelectric generating units range in size from less than 1 megawatt to 700 megawatts. Because of their ability to start quickly and make rapid changes in power output, hydroelectric generating units are suitable for serving peak loads and providing spinning reserve power, as well as serving baseload requirements. 
Figure 20. Hydroelectric Power System with Reservoir

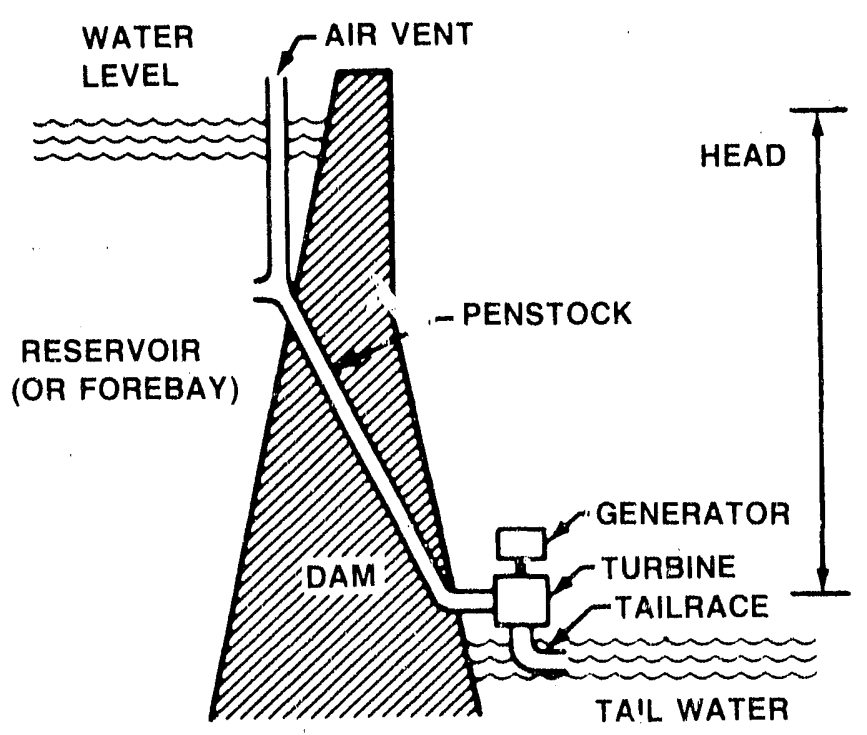

Source: U.S. Department of Energy, Energy Deskbook (June 1982), p. 188.

Another kind of hydroelectric power generation is the pumped storage hydroelectric system. Pumped storage hydroelectric plants use the same principle for generation of power as the conventional hydroelectric operations based on falling water and river current. However, all or part of the water is made available for repeated use by pumping it from a lower to an upper reservoir. In the pumped storage operation, low-cost off-peak energy is used to pump water to an upper reservoir where it is stored as potential energy. The water is then released to flow back down through the turbine generator to produce electricity during periods of high demand for electricity.

Other Generating Units. Other methods of electric power generation, which presently contribute only small amounts to total power production, have potential for expansion. These include geothermal, refuse, waste heat, waste steam, solar, wind, and wood. Geothermal power comes from heat energy buried deep beneath the surface of the earth. Although most of this heat is at depths beyond current drilling methods, in some areas of the country magma flows close enough to the surface of the earth to produce steam. That steam can then be harnessed for use in conventional steam-turbine plants. Solar power is derived from the energy (both light and heat) of the sun. Photovoltaic conversion generates electric power directly from the light of the sun; whereas, solar-thermal electric generators use the heat from the sun to produce steam to drive turbines. Wind power is derived from the conversion of the energy contained in wind into electricity. A wind turbine is similar to a typical wind mill. However, because of the intermittent nature of sunlight and wind, high capacity utilization factors cannot be achieved for these plants. Several electric utilities have incorporated wood and refuse (for example, municipal waste, corn cobs, and oats) as energy sources for producing electricity at their power plants. These sources replace fossil fuels in the boiler. The combustion of wood and refuse creates steam that is typically used in conventional steam-electric plants.

\section{Generator Rating}

The rating of a generator is a measure of its ability to produce electricily. Generators are rated by nameplate capacity. The nameplate capacity is the full-load continuous rating of the generator under specified conditions, as designated by the manufacturer, and is usually indicated on a metal plate attached physically to the generator. Net capability is the steady hourly output that the generating unit is expected to supply to the system load as demonstrated by test procedures. The capability of the generating unit in the summer is generally less than in the winter due to high ambient-air and cooling-water temperatures, which cause generating units to be less efficient. The measure used in this publication is net summer capability. The nameplate capacity of a generator is generally greater than its net capability. Nameplate capacity is a gross rating of the output of the generator whereas net capability is the output of the generating unit based on system load deducting the requirements of the plant.

\section{Planned Generating Unit Additions}

Electric utilities must provide a continuous and dependable supply of electricity to their consumers. They must maintain a balance between supply and demand and, at the same time, maintain an adequate reserve margin to ensure system reliability. To accomplish this, electric utilities continually assess their need for new electric generating capability. Summaries of the plans of electric utilities for additions of generating capability in new units at the national, regional, and State levels from 1990 through 1999 are given in Tables 5, 10, and 11.

The most important factor in determining the need for new electric generating capability is growth in the demand for electricity. (Growth in demand is influenced primarily by economic forces, for example, the Gross National Product and the cost of fuels and electricity). Electric utilities constantly monitor their peak demands and energy requirements and usually update their forecasts of these annually. Electric utilities use various methodologies to forecast annual peak demand and energy requirements, from which they determine the amount of generating capacity that is needed to meet 
expected demand. Two additional factors that contribute to the need for new generating capability are the retirements of older generators and the decreasing efficiency of aging generating units.

Before deciding to add new capability, electric utilities consider other options that may be more cost-effective in meeting future demand. Also, they may choose to combine the addition of new capability with other options that include repowering and/or life extension of their existing capability, load management programs and/or purchases of power from nonutilities.

Electric utilities are considering repowering as a costeffective means of meeting future energy requirements. Several repowering technologies have been developed. Repowering generally consists of modifying old coalfired electric generating units by replacing the boiler with a new combustion technology which results in hetler performance and an increase in capacily. Several repowering technologies were reported as part of the 10-year construction plans of utilities. In some cases, an existing stcam-electric plant is reconfigured as a combined eycle plant by adding a gas turbine. Several clectric utilities have already completed this kind of repowering and more of it is planned in their 10-year construction plans. (Other technologies repower with clean coal technology. Repowering witb clean coal technology includes replacing the furnace and boiler in an old plant with new, cleaner-burning, highefficient coal combustion or gasification technology. Although utilities are considering their reported plans for implementing these technologies as "demonstration" projects, test programs have shown that these technologies do have dependability for future use by electrir: utilities in the United States.

Although life extension (plant refurbishment) adds little, if any, new generating capacity, some utilities have chosen to refurbish their plants since refurbishment can add about 20 years of life to aged plants nearing retirement and is less expensive than repowering.

Nonutilities are expected to supply a significant portion of the generating capacity needed to meet energy requirements of electric utilities over the next 10 years. By $20(0)$, nonutility capacity is projected to total $66,0(0)$ megawatts. ${ }^{12}$ About one-third of this capacity is expected to be sold to the electric utility grid.

With concerns about future demand, in particular, future peak demands, utilities are studying and implementing ways to get customers to reduce or shift loads. These techniques include allowing customers to have more choice in setting the cost of their energy, controlling their energy use and at the same time reducing overall demand. Reducing demand delays the need for new generating capacity. The North American Electric Reliability Council (NERC) reported expected load management programs equivalent to approximately 11,000 megawatts in 1989. ${ }^{1 *}$ By 1998, NERC expects this number to be over more than $17,0(0)()$ megawatts.

Once the decision has been made to add capability either by building new power plants or adding new generating units at existing power plants, the type of capability to be added is determined by matching the load to be served with the appropriate type of generating unit. If the needs of a utility are limited to capability requiled to meet peak demand, then gas turbine, internal-combustion, or pumped-storage hydroclectric generating units are likely to be the choice for new additions. To meet other energy requirements (intermediate and base loads), the options available of the utility include the addition of steam-electric, combinedcycle, or conventional-hydroelectric generating imits.

Although utilities are responding to the various options to maintain an adequate electric power supply, the options are not without limitations. Even with the imple. mentation of the above options, the Energy Information Administration estimates that electric utilities will build $66,()(x)$ megawatts of currently unammonnced or umplanned capacity by $20(0)$. Combined cycle and gas turbines, which require relatively short construction time, are expected to account for $52,(x)$ megawatts of the unplanned or unannounced capacity."

\section{Data Sources}

The following tables contain a summary of the number of electric generatoris and electric generating capability at the national, regional, and State levels for the period 1086 through 1989 , as well as, capability additions planned by electric utilities for 1990 through 1969. The number of generators are reported since this is a count of the physical equipment rather than generating units, which are a combination of onc or more boilers, turbines, and generators operated together to produce electricity. Data in the tables were obtained from the Form EIA-860, "Annual Electric Generator Report." Data are reported annually on the Form EIA-860) by approximately 900 electric utilities in the United States that operate power plants or plan to operate power plants within 10 years of the reporting year. These 10-year plans include gentrating units that are under construction or in various stages of planning at the time data are submitted. More detailed statistics from the Form EIA-860) are published in the Inventory of Power Plants in the United States."

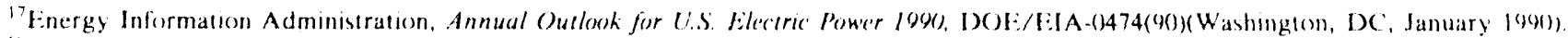

${ }^{1 \mathrm{k}} \mathrm{North}$ American Electric Reliability Council, 1989 E:lectricty Supply and Demand. (Princeton, New Jersey, ()ciober 1989).

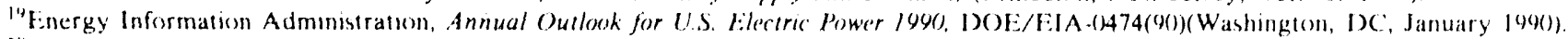

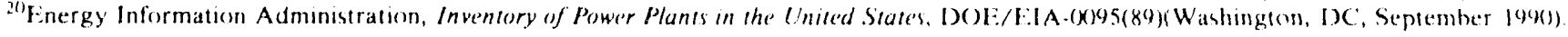


Table 3. Generating Capability by Prime Mover and Primary Energy Source, 1986-1989 (Megawatts)

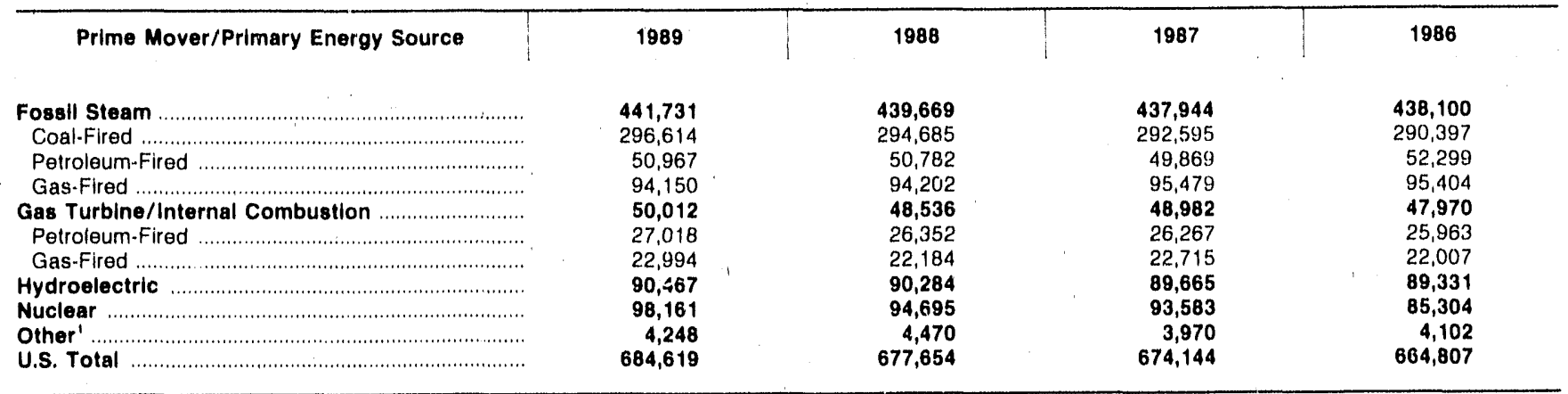

I Includes geothermal, refuse, waste heat, waste steam, solar, wind, and wood

Notes: - Totals may not equal sum of components because of independent rounding. -Generating capability is net summer capability. Source: Energy information Administration, Form ElA-860, "Annual Electric Generator Report."

Table 4. Summary of Capability Additions and Retirements, and Total Operable Capability by Energy Source, 1989

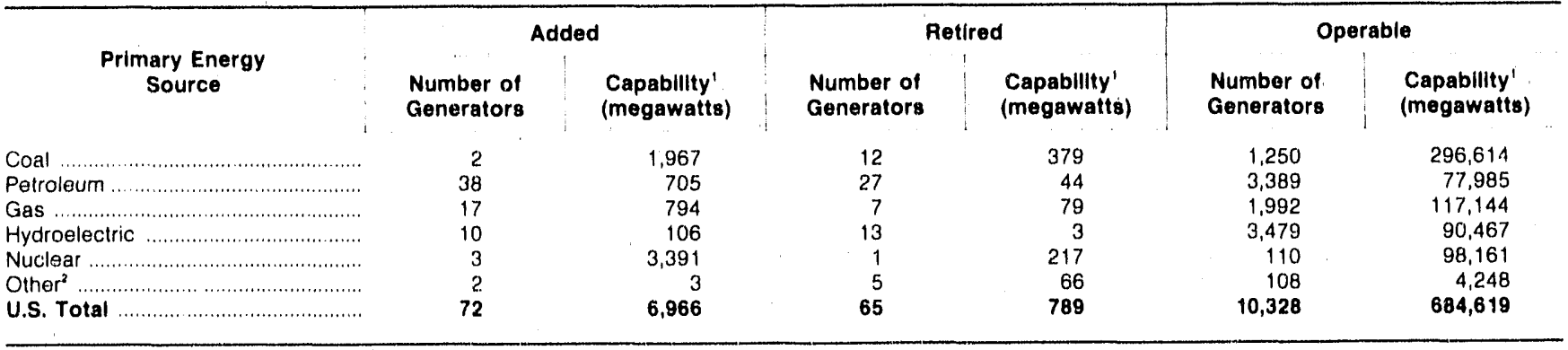

1 Net summer capability.

2 Includes geothermal, refuse, waste heat, waste steam, solar, wirid, and wood

Notes: - Totals may not equal stin of components because of independent rounding. - Total capability cannot be calculated from the prior year's capability by adjusting for retirements and newly added capability because capability ratings for independent generators change each year and generators are purchased from or sold to nonutilities.

Source: Energy Information Administration, Form ElA-860, "Annual Electric Generator Report." 
Table 5. Planned Capability Additions by Energy Source, 1990-1999

\begin{tabular}{|c|c|c|c|c|c|c|}
\hline \multirow[b]{2}{*}{ Year } & \multicolumn{2}{|c|}{ Total } & \multicolumn{2}{|c|}{ Petroleum \& Gas } & \multicolumn{2}{|c|}{ Coal } \\
\hline & $\begin{array}{l}\text { Number of } \\
\text { Generators }\end{array}$ & $\begin{array}{c}\text { Capability' } \\
\text { (megawatts) }\end{array}$ & $\begin{array}{l}\text { Number of } \\
\text { Generators }\end{array}$ & $\begin{array}{l}\text { Capabllity' } \\
\text { (megawatts) }\end{array}$ & $\begin{array}{l}\text { Number of } \\
\text { Generators }\end{array}$ & $\begin{array}{l}\text { Capability' } \\
\text { (megawatt8) }\end{array}$ \\
\hline $1990 \ldots \ldots \ldots \ldots$ & 43 & 4,141 & 23 & 722 & 4 & 1,006 \\
\hline 1991 & 38 & 6,238 & 16 & 668 & 4 & 2,735 \\
\hline 1992 & 35 & 2,460 & 14 & 897 & 2 & 518 \\
\hline $1993 \ldots \ldots \ldots \ldots \ldots \ldots \ldots \ldots$ & 34 & 2,443 & 22 & 1,484 & 1 & 393 \\
\hline $1994 \ldots \ldots \ldots \ldots \ldots \ldots \ldots \ldots \ldots \ldots$ & 44 & 3,218 & 19 & $i, 305$ & 2 & 1,143 \\
\hline $1995 \ldots \ldots \ldots \ldots \ldots \ldots \ldots \ldots$ & 39 & 7,294 & 21 & 1,867 & 5 & 2,780 \\
\hline $1996 \ldots \ldots \ldots \ldots \ldots \ldots \ldots \ldots$ & 37 & 4,692 & 30 & 2,367 & 4 & 2,256 \\
\hline 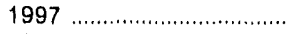 & 29 & 3,706 & 21 & 1,993 & 7 & $.1,710$ \\
\hline $1998 \ldots \ldots \ldots \ldots \ldots \ldots \ldots \ldots$ & 35 & 4,169 & 16 & 1,669 & 7 & 2,372 \\
\hline \multirow[t]{3}{*}{1999} & 34 & 2,871 & 26 & 1,925 & 3 & 900 \\
\hline & \multicolumn{2}{|c|}{ Nuclear } & \multicolumn{2}{|c|}{ Hydroelectric } & \multicolumn{2}{|c|}{ Other ${ }^{2}$} \\
\hline & $\begin{array}{l}\text { Number of } \\
\text { Generators }\end{array}$ & $\begin{array}{l}\text { Capability' } \\
\text { (megawatts) }\end{array}$ & $\begin{array}{l}\text { Number of } \\
\text { Generators }\end{array}$ & $\begin{array}{l}\text { Capabillty' } \\
\text { (megawatts) }\end{array}$ & $\begin{array}{l}\text { Number of } \\
\text { Generstors }\end{array}$ & $\begin{array}{l}\text { Capabillty' } \\
\text { (megawatts) }\end{array}$ \\
\hline $1990 \ldots \ldots \ldots \ldots \ldots \ldots \ldots \ldots \ldots \ldots$ & 2 & 2,300 & 13 & 52 & 1 & 60 \\
\hline $1991 \ldots \ldots \ldots \ldots \ldots \ldots \ldots \ldots$ & 2 & 2,320 & 16 & 515 & 0 & 0 \\
\hline $1992 \ldots, \ldots \ldots \ldots \ldots \ldots \ldots \ldots \ldots$ & 0 & 0 & 16 & 983 & 3 & 62 \\
\hline $1993 \ldots \ldots \ldots \ldots \ldots \ldots$ & 0 & 0 & 9 & 366 & 2 & 200 \\
\hline $1994 \ldots \ldots \ldots \ldots \ldots \ldots \ldots \ldots \ldots$ & 0 & 0 & 18 & 400 & 5 & 370 \\
\hline $1995 \ldots \ldots \ldots$ & 1 & 1,170 & 9 & 1,036 & 3 & 441 \\
\hline $1996 \ldots \ldots \ldots \ldots \ldots \ldots \ldots \ldots \ldots$ & 0 & 0 & 3 & 69 & 0 & 0 \\
\hline 1997 & 0 & 0 & 1 & 2 & 0 & 0 \\
\hline $1998 \ldots \ldots \ldots \ldots \ldots \ldots \ldots$ & 0 & 0 & 10 & 34 & 2 & 94 \\
\hline $1999 \ldots \ldots \ldots \ldots \ldots \ldots \ldots \ldots \ldots \ldots$ & 0 & 0 & 5 & 46 & 0 & 0 \\
\hline
\end{tabular}

' Net summer capability.

2 Includes waste heat and solar.

Note: oTotals may not equal sum of components because of independent rounding.

Source: Energy Information Administration, Form ElA-860, "Annual Electric Generator Reporl." 
Table 6. Generating Capability by Census Division and State, 1989 and 1988

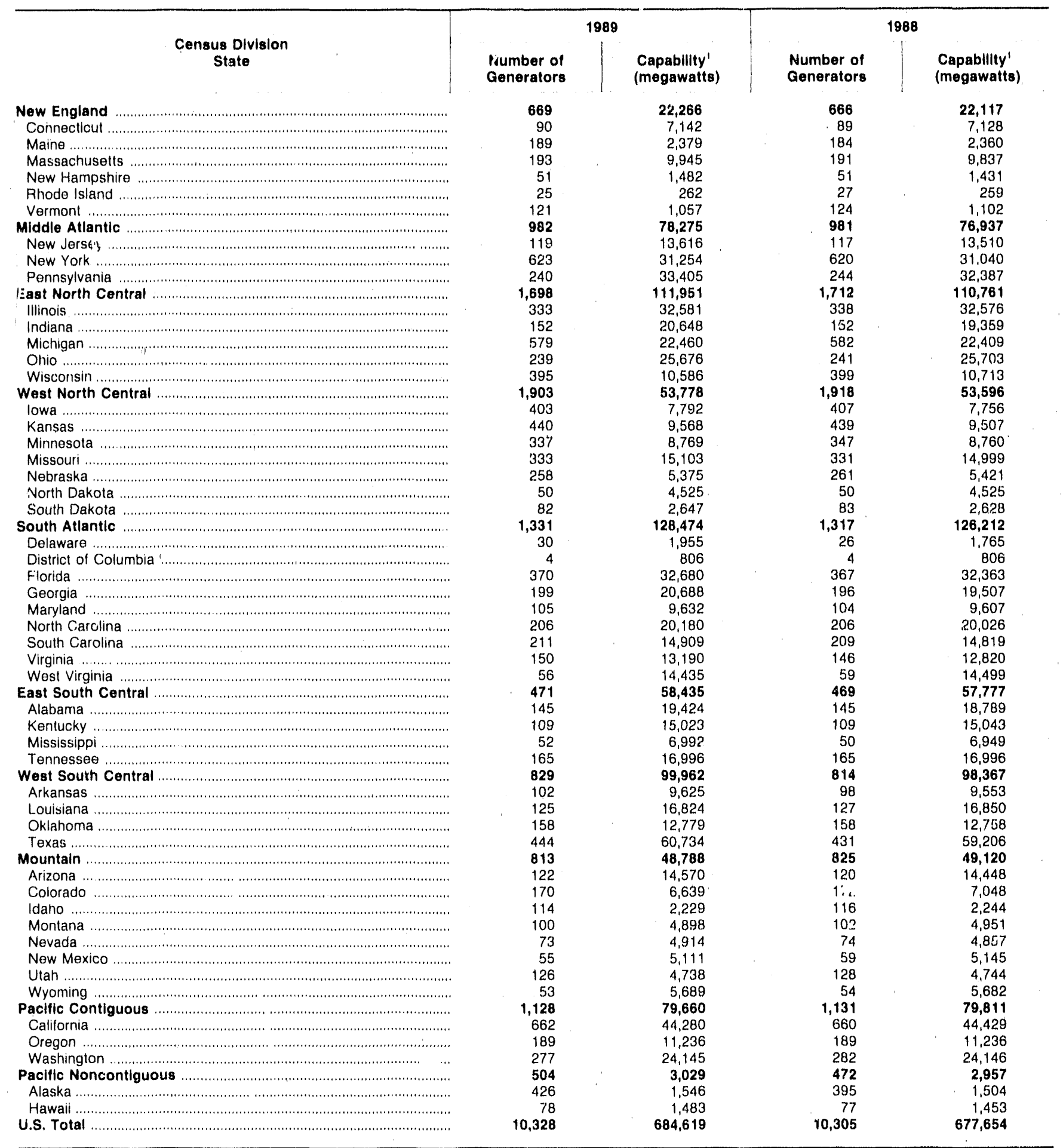

1 Net summer capability.

Note: Totals may not equal sum of components because of independent rounding.

Source: Energy information Administration, Form EIA-860, "Annual Electric Generator Report." 
Table 7. Coal-Fired, Nuclear, Hydroelectric, and Other Generating Capability by Census Division and State, 1989

\begin{tabular}{|c|c|c|c|c|c|c|c|c|}
\hline \multirow{3}{*}{$\begin{array}{c}\text { Census Dlvision } \\
\text { State }\end{array}$} & \multirow{2}{*}{\multicolumn{2}{|c|}{ Coal }} & \multicolumn{2}{|c|}{ Nuclear } & \multicolumn{2}{|c|}{ Hydroelectric } & \multicolumn{2}{|c|}{ Other' } \\
\hline & & & & 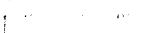 & & & & \\
\hline & $\begin{array}{l}\text { Number of } \\
\text { Generators }\end{array}$ & $\begin{array}{c}\text { Capability } \\
\text { (megawatts) }\end{array}$ & $\begin{array}{l}\text { Number of } \\
\text { Generators }\end{array}$ & $\begin{array}{l}\text { Capabllity } \\
\text { (megawatts) }\end{array}$ & $\begin{array}{l}\text { Number of } \\
\text { Generators }\end{array}$ & $\begin{array}{l}\text { Capabillty } \\
\text { (megawatts) }\end{array}$ & $\begin{array}{l}\text { Number of } \\
\text { Generators }\end{array}$ & $\begin{array}{c}\text { Capablilty" } \\
\text { (megawatts) }\end{array}$ \\
\hline New England ................. & 15 & 2,755 & 8 & 5,406 & 365 & 3,105 & 5 & 229 \\
\hline 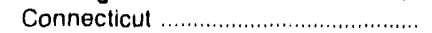 & 1 & 385 & 4 & 3,217 & 35 & 141 & 2 & 64 \\
\hline Maine & 0 & 0 & 1 & 860 & 131 & 390 & 0 & 0 \\
\hline 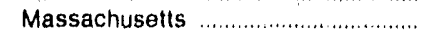 & 9 & 1,750 & 2 & 834 & 64 & 1,853 & 2 & 118 \\
\hline New Hampshire ................................ & 5 & 620 & 0 & 0 & 39 & 320 & 0 & 0 \\
\hline Rhode Island & 0 & 0 & 0 & 0 & 1 & 1 & 0 & 0 \\
\hline Vermont & 0 & 0 & 1 & 496 & 95 & 399 & 1 & 47 \\
\hline Middle Atlantlc & 106 & 23,042 & 19 & 17,376 & 377 & 7,277 & 2 & 189 \\
\hline 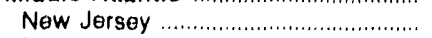 & 9 & 1,652 & 4 & 3,863 & 3 & 330 & 1 & 120 \\
\hline (2) & 32 & 3,882 & 6 & 4,766 & 329 & 5,070 & 0 & 0 \\
\hline 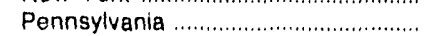 & 65 & 17,508 & 9 & 8,747 & 45 & 1,877 & 1 & 69 \\
\hline 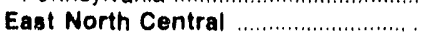 & 397 & 75,541 & 23 & 20,215 & 493 & 2,874 & 11 & 258 \\
\hline Illinois & 61 & 14,934 & 13 & 12,609 & 14 & 10 & 0 & 0 \\
\hline Indiana & 80 & 19,579 & 0 & 0 & 20 & 76 & 0 & 0 \\
\hline 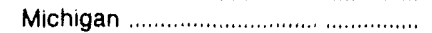 & 82 & 12,029 & 5 & 4,045 & 247 & 2,210 & 0 & 0 \\
\hline Ohio & 120 & 21,866 & 2 & 2,041 & 7 & 120 & 4 & 154 \\
\hline 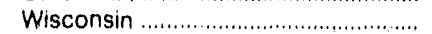 & 54 & 7,132 & 3 & 1,520 & 205 & 458 & 7 & 102 \\
\hline 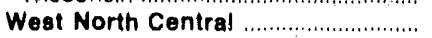 & 207 & 34,519 & 8 & 5,574 & 167 & 3,859 & 15 & 109 \\
\hline lowa & 53 & 5,855 & 1 & 520 & 24 & 124 & 3 & 21 \\
\hline Kansas & 20 & 5,064 & 1 & 1,135 & 7 & 2 & 2 & 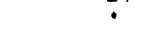 \\
\hline Minnesola & 55 & 5,716 & 3 & 1,540 & 54 & 137 & 10 & 88 \\
\hline 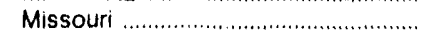 & 47 & 10,649 & 1 & 1,125 & 29 & 1,062 & 0 & 0 \\
\hline Nebraska & 12 & 2,869 & 2 & 1,254 & 20 & 168 & 0 & 0 \\
\hline North Dakota & 14 & 3,876 & 0 & 0 & 5 & 545 & 0 & 0 \\
\hline South Dakota & 6 & 489 & 0 & 0 & 28 & 1,821 & 0 & 0 \\
\hline South Atlantlc & 215 & 63,569 & 27 & 23,614 & 452 & 10,318 & 16 & 630 \\
\hline 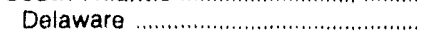 & 5 & 919 & 0 & 0 & 0 & 0 & 0 & 0 \\
\hline District of Columbia ........................ & 0 & 0 & 0 & 0 & 0 & 0 & 0 & 0 \\
\hline 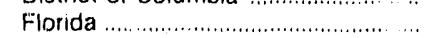 & 29 & 9,898 & 5 & 3,825 & 7 & 46 & 7 & 482 \\
\hline 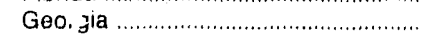 & 39 & 12,951 & 4 & 3,703 & 113 & 2,465 & 0 & 0 \\
\hline 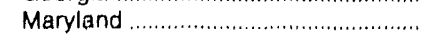 & 14 & 3,972 & 2 & 1,650 & 13 & 428 & 0 & 0 \\
\hline North Carolina & 47 & 12,470 & 5 & 4,698 & 105 & 1,979 & 3 & 80 \\
\hline South Carolina & 24 & 4,818 & 7 & 6,346 & 121 & 2,183 & 3 & 09 \\
\hline Virginia & 24 & 4,225 & 4 & 3,392 & 71 & 3,109 & 3 & 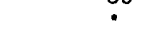 \\
\hline West Virginia & 33 & 14,315 & 0 & 0 & 22 & 108 & 0 & 0 \\
\hline East South Central & 144 & 36,528 & 8 & 8,291 & 205 & 7,472 & 0 & 0 \\
\hline Alabama & 41 & 11,171 & 5 & 4,853 & 89 & 2,934 & 0 & 0 \\
\hline 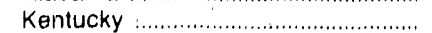 & 57 & 13,818 & 0 & 0 & 30 & 795 & 0 & 0 \\
\hline 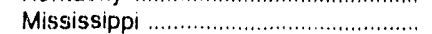 & 9 & 2,250 & 1 & 1,142 & 0 & 0 & 0 & 0 \\
\hline 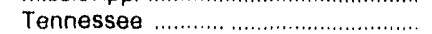 & 37 & 9,289 & 2 & 2,296 & 86 & 3,743 & 0 & 0 \\
\hline West South Central & 54 & 30,421 & 6 & 6,206 & 122 & 2,931 & 7 & 506 \\
\hline Arkansás & 5 & 3,817 & 2 & 1,694 & 40 & 1,287 & 0 & 0 \\
\hline 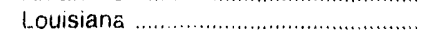 & 6 & 3,343 & 2 & 2,011 & 0 & 0 & 1 & 203 \\
\hline Oklahoma & 10 & 4,828 & 0 & 0 & 37 & 1,003 & 0 & 0 \\
\hline Texas & 33 & 18.433 & 2 & 2,501 & 45 & 640 & 6 & 303 \\
\hline Mountain & 104 & 28,377 & 3 & 3,663 & 406 & 9,954 & 9 & 39 \\
\hline Arizona & 13 & 4,756 & 3 & 3,663 & 38 & 2,739 & 0 & 0 \\
\hline Colorado & 31 & 4,942 & 0 & 0 & 48 & 1,076 & 0 & 0 \\
\hline ddaho & 0 & 0 & 0 & 0 & 110 & 2,174 & 0 & 0 \\
\hline Montana & 6 & 2,260 & 0 & 0 & 84 & 2,506 & 7 & 13 \\
\hline Nevada & 8 & 2,692 & 0 & 0 & 17 & 1,001 & 0 & 0 \\
\hline 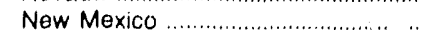 & 13 & 3,894 & 0 & 0 & 6 & 56 & 1 & 5 \\
\hline Utah & 14 & 4,424 & 0 & 0 & 78 & 141 & 1 & 21 \\
\hline Wyoming & 19 & 5,410 & 0 & 0 & 25 & 261 & 0 & $\begin{array}{r}2 \\
0\end{array}$ \\
\hline Pacilic Contlguous & 3 & 1,806 & 8 & 7,815 & 845 & 42,438 & 37 & 2,204 \\
\hline California & 0 & $\begin{array}{r}1,000 \\
0\end{array}$ & 6 & 5,611 & 414 & 12,490 & 34 & $\begin{array}{l}4,204 \\
1,998\end{array}$ \\
\hline Oregon & 1 & 530 & 1 & 1,104 & 175 & 8,988 & 2 & 160 \\
\hline Washington & 2 & 1,276 & 1 & 1,100 & 256 & 20,959 & 1 & 46 \\
\hline Paclfic Noncontlguous & 5 & 56 & 0 & 0 & 47 & $\begin{array}{r}239 \\
239\end{array}$ & 6 & 85 \\
\hline 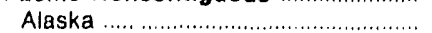 & 5 & 56 & 0 & 0 & 43 & 236 & 5 & $B 1$ \\
\hline 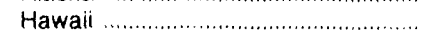 & 0 & 0 & 0 & 0 & 4 & 3 & 1 & 4 \\
\hline U.S. Total & 1,250 & 296,614 & 110 & 98,161 & 3,479 & 90,467 & 108 & 4,248 \\
\hline
\end{tabular}

i Includes geothermal, reluse, waste heat, waste steam, solar, wind, and wood

2 Net summer copability.

- Value less than 0.5

Note: - Totals may not equal sum of components because of independent rounding.

Source: Energy Information Adrninistration, Form ElA-860, "Annual Electric Generator Report." 
Table 8. Petroleum-, Gas-, and Dual-Fired Steam Generating Capability by Census Division and State, 1989

\begin{tabular}{|c|c|c|c|c|c|c|c|c|}
\hline \multirow{2}{*}{$\begin{array}{c}\text { Census Division } \\
\text { State }\end{array}$} & \multicolumn{2}{|c|}{ Petroleum } & \multicolumn{2}{|c|}{ Gas } & \multicolumn{2}{|c|}{$\begin{array}{c}\text { Dual-Fired } \\
\text { Petroleum/Gas }\end{array}$} & \multicolumn{2}{|c|}{$\begin{array}{c}\text { Total } \\
\text { Petroleum and Gas }\end{array}$} \\
\hline & $\begin{array}{l}\text { Number of } \\
\text { Generators }\end{array}$ & $\begin{array}{l}\text { Capabllity' } \\
\text { (megawatts) }\end{array}$ & $\begin{array}{l}\text { Number of } \\
\text { Generg'cors }\end{array}$ & $\begin{array}{c}\text { Capabillty' } \\
\text { (megawatts) }\end{array}$ & $\begin{array}{l}\text { Number of } \\
\text { Generators }\end{array}$ & $\begin{array}{c}\text { Capabillity' } \\
\text { (megawatts) }\end{array}$ & $\begin{array}{l}\text { Number of } \\
\text { Generators }\end{array}$ & $\begin{array}{l}\text { Capabillty' } \\
\text { (megawatts) }\end{array}$ \\
\hline 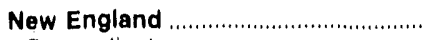 & 53 & 6,994 & 0 & 0 & 23 & 2,089 & 76 & 9,083 \\
\hline 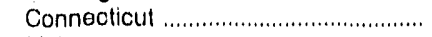 & 22 & 2,418 & 0 & 0 & 2 & 528 & 24 & 2,946 \\
\hline 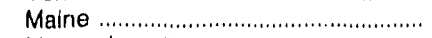 & 14 & 1,053 & 0 & 0 & 0 & 0 & 14 & 1,053 \\
\hline 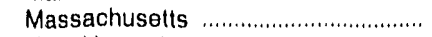 & 15 & 3,075 & 0 & 0 & 15 & 1,328 & 30 & 4,404 \\
\hline 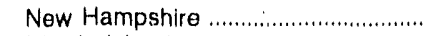 & 2 & 447 & 0 & 0 & 0 & 0 & 2 & 447 \\
\hline 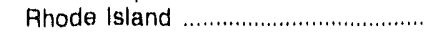 & 0 & 0 & 0 & 0 & 6 & 233 & 6 & 233 \\
\hline 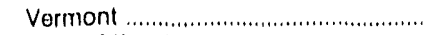 & 0 & 0 & 0 & 0 & 0 & 0 & 0 & 0 \\
\hline 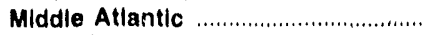 & 54 & 11,368 & 0 & 0 & 51 & 9,205 & 105 & 20,573 \\
\hline 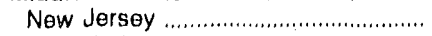 & 16 & 1,599 & 0 & 0 & 16 & 1,906 & 32 & 3,505 \\
\hline 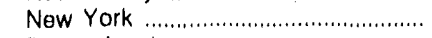 & 25 & 6,377 & 0 & 0 & 35 & 7,299 & 60 & 13,676 \\
\hline 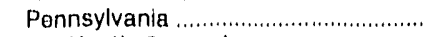 & 13 & 3,392 & 0 & 0 & 0 & 0 & 13 & $3,39 ?$ \\
\hline 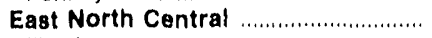 & 29 & 6,026 & 9 & 228 & 16 & 734 & 54 & 6,988 \\
\hline 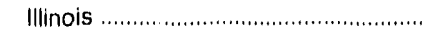 & 14 & 3,116 & 2 & 19 & 9 & 304 & 25 & 3,439 \\
\hline Indiana & 5 & 193 & 2 & 120 & 7 & 0 & 7 & 313 \\
\hline 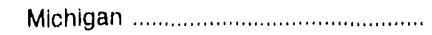 & 6 & 2,474 & 1 & $\theta$ & 4 & 401 & 11 & 2,884 \\
\hline 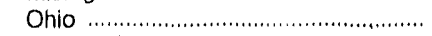 & 4 & 243 & 0 & 0 & 1 & 17 & 5 & 260 \\
\hline 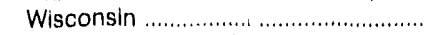 & 0 & 0 & 4 & 80 & 2 & 12 & 6 & 92 \\
\hline 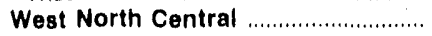 & 2 & 50 & 16 & $\$ 44$ & 76 & 2,303 & 94 & 2,797 \\
\hline 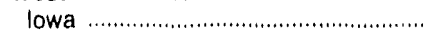 & 0 & 0 & 2 & 44 & 3 & 60 & 5 & 104 \\
\hline 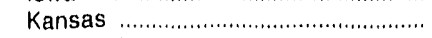 & 0 & 0 & 6 & 167 & 52 & 1,786 & 58 & 1,953 \\
\hline 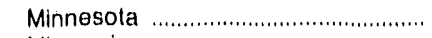 & 2 & 50 & 0 & 0 & 5 & 56 & 7 & 106 \\
\hline 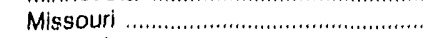 & $\overline{0}$ & 0 & 4 & 101 & 4 & 79 & 8 & 180 \\
\hline 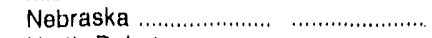 & 0 & 0 & 4 & 132 & 11 & 259 & 15 & 391 \\
\hline 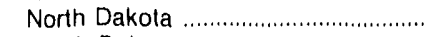 & 0 & 0 & 0 & 0 & 0 & 0 & 0 & 0 \\
\hline 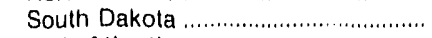 & 0 & 0 & 0 & 0 & 1 & 64 & 1 & 64 \\
\hline 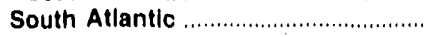 & 55 & 8,284 & 5 & 303 & 83 & 11,470 & 143 & 20,056 \\
\hline Delaware & 2 & 54 & 0 & 0 & 6 & 684 & 8 & 738 \\
\hline District of Columbia ............................ & 2 & 550 & 0 & 0 & 0 & 0 & 2 & 550 \\
\hline 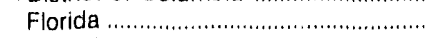 & 31 & 5,508 & 5 & 303 & 61 & 8,013 & 97 & 13,824 \\
\hline 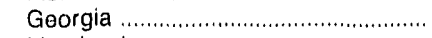 & 2 & 118 & 0 & 0 & 8 & 421 & 10 & 539 \\
\hline 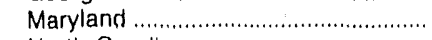 & 13 & 1,033 & 0 & 0 & 4 & 1,439 & 17 & 2,472 \\
\hline 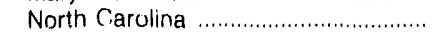 & 0 & 0 & 0 & 0 & 0 & 0 & 0 & 0 \\
\hline 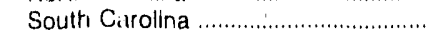 & 2 & 92 & 0 & 0 & 3 & 94 & 5 & 186 \\
\hline 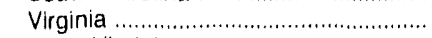 & 3 & 929 & 0 & 0 & 1 & 818 & 4 & 1,747 \\
\hline 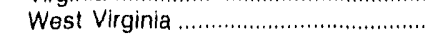 & 0 & 0 & 0 & 0 & 0 & 0 & 0 & 0 \\
\hline 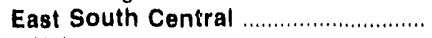 & 2 & 59 & 4 & 328 & 27 & 3,210 & 33 & 3,597 \\
\hline 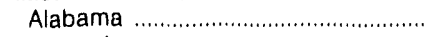 & 0 & 0 & 0 & 0 & 1 & 50 & 1 & 50 \\
\hline 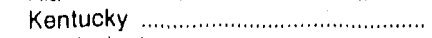 & 2 & 59 & 1 & 115 & 0 & 0 & 3 & 174 \\
\hline 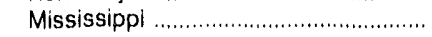 & 0 & 0 & 3 & 213 & 26 & 3,161 & 29 & 3,374 \\
\hline 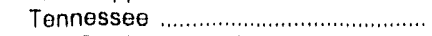 & 0 & 0 & 0 & 0 & 0 & 0 & 0 & 0 \\
\hline 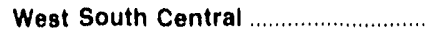 & 0 & 0 & 61 & 8,148 & 266 & 47,416 & 327 & 55,564 \\
\hline 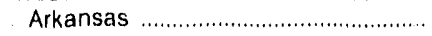 & 0 & 0 & 0 & 0 & 16 & 2,515 & 16 & 2,515 \\
\hline 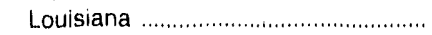 & 0 & 0 & 17 & 662 & 59 & 10,385 & 76 & 11,047 \\
\hline 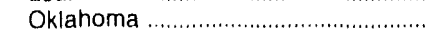 & 0 & 0 & 11 & 677 & 22 & 5,427 & 33 & 6,104 \\
\hline 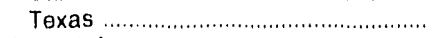 & 0 & 0 & 33 & 6,808 & 169 & 29,089 & 202 & 35,897 \\
\hline 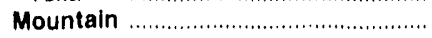 & 0 & 0 & 7 & 512 & 73 & 3,272 & 80 & 3,784 \\
\hline 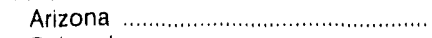 & 0 & 0 & 0 & 0 & 25 & 1,607 & 25 & 1,607 \\
\hline 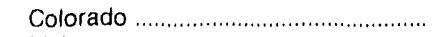 & 0 & 0 & 1 & 8 & 17 & 268 & 18 & 276 \\
\hline 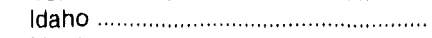 & 0 & 0 & 0 & 0 & 0 & 0 & 0 & 0 \\
\hline Montana & 0 & 0 & 0 & 0 & 1 & 70 & 1 & 70 \\
\hline 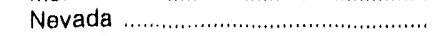 & 0 & 0 & 1 & 113 & 8 & 618 & 9 & 731 \\
\hline 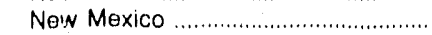 & 0 & 0 & 5 & 391 & 21 & 644 & 26 & 1,035 \\
\hline 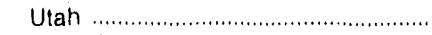 & 0 & 0 & 0 & 0 & 1 & 66 & 1 & 56 \\
\hline 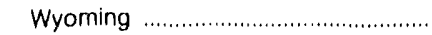 & 0 & 0 & 0 & 0 & 0 & 0 & 0 & 0 \\
\hline 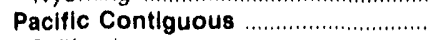 & 2 & 86 & 1 & 358 & 115 & 21,079 & 118 & 21,523 \\
\hline 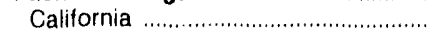 & $\overline{0}$ & 0 & 1 & 358 & 115 & 21,079 & 116 & 21,437 \\
\hline Oregon & 0 & 0 & 0 & 0 & 0 & 0 & 0 & 0 \\
\hline 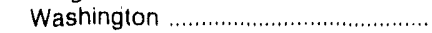 & 2 & 86 & 0 & 0 & 0 & 0 & 2 & 86 \\
\hline Pacific Noncontiguous ....................... & 25 & 1,151 & 0 & 0 & 0 & 0 & 25 & 1,151 \\
\hline 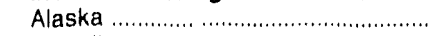 & 0 & 0 & 0 & 0 & 0 & 0 & 0 & 0 \\
\hline 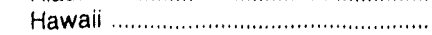 & 25 & 1,151 & 0 & 0 & 0 & 0 & 25 & 1,151 \\
\hline 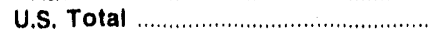 & 222 & 34,018 & 103 & 10,320 & 730 & 100,779 & 1,055 & 145,117 \\
\hline
\end{tabular}

1 Nel summer capability

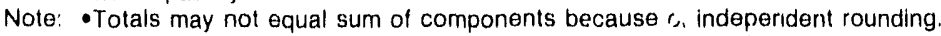

Source: Energy Intormation Administration, Form EIA.-860, "Annual Electric Generator Report." 
Table 9. Petroleum-, Gas-, and Dual-Fired Gas Turbine/Internal Combustion Generating Capability by Census Division and State, 1989

\begin{tabular}{|c|c|c|c|c|c|c|c|c|}
\hline \multirow{2}{*}{$\begin{array}{c}\text { Census Division } \\
\text { State }\end{array}$} & \multicolumn{2}{|c|}{ Petroleum } & \multicolumn{2}{|c|}{ Gas } & \multicolumn{2}{|c|}{$\begin{array}{l}\text { Dual-Fired } \\
\text { Petroleum/Gas }\end{array}$} & \multicolumn{2}{|c|}{$\begin{array}{l}\text { Total } \\
\text { Potroleum and Gas }\end{array}$} \\
\hline & $\begin{array}{l}\text { Number of } \\
\text { Generators }\end{array}$ & $\begin{array}{l}\text { Capability' } \\
\text { (megawatts) }\end{array}$ & $\begin{array}{l}\text { Number of } \\
\text { Generators }\end{array}$ & $\begin{array}{l}\text { Ca } \\
\text { (m gallity' }\end{array}$ & $\begin{array}{l}\text { Number of } \\
\text { Generators }\end{array}$ & $\begin{array}{c}\text { Capabillty' } \\
\text { (megawatts) }\end{array}$ & $\begin{array}{l}\text { Number of } \\
\text { Generators }\end{array}$ & $\begin{array}{l}\text { Capabillty' } \\
\text { (megawatts) }\end{array}$ \\
\hline New England & 183 & 1,307 & 0 & 0 & 17 & 381 & 200 & 1,688 \\
\hline 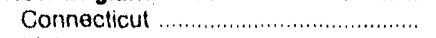 & 24 & 389 & 0 & 0 . & 0 & 0 & 24 & 389 \\
\hline 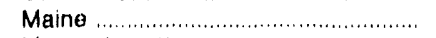 & 43 & 75 & 0 & 0 & 0 & 0 & 43 & 75 \\
\hline 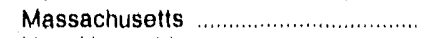 & 70 & 625 & 0 & 0 & 16 & 361 & 86 & 986 \\
\hline New Hampshire ................................... & 4 & 76 & 0 & 0 & 1 & 19 & 5 & 95 \\
\hline 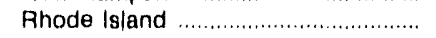 & 18 & 27 & 0 & 0 & 0 & 0 & 18 & 27 \\
\hline 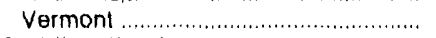 & 24 & 115 & 0 & 0 & 0 & 0 & 24 & 415 \\
\hline 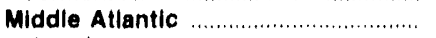 & 243 & 5,571 & 7 & 115 & 123 & 4,131 & 373 & 9,818 \\
\hline 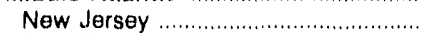 & 30 & 1,919 & 3 & 51 & 37 & 2,176 & 70 & 4,146 \\
\hline 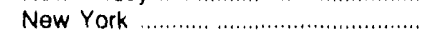 & 117 & 2,083 & 3 & 45 & 76 & 1,732 & 196 & 3,860 \\
\hline 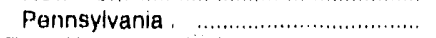 & 96 & 1,570 & 1 & 19 & 10 & 223 & 107 & 1,812 \\
\hline East North Central & 412 & 3,012 & 32 & 698 & 276 & 2,367 & 720 & 6,077 \\
\hline 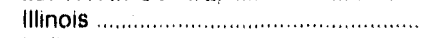 & 76 & 562 & 5 & 35 & 139 & 991 & 220 & 1,588 \\
\hline 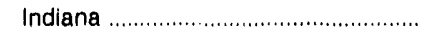 & 26 & 286 & 8 & 246 & 11 & 148 & 45 & 679 \\
\hline 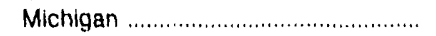 & 159 & 567 & 18 & 403 & 57 & 323 & 234 & 1,292 \\
\hline Ohio & 63 & 819 & 1 & 14 & 37 & 402 & 101 & 1,235 \\
\hline 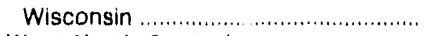 & 88 & 779 & 0 & 0 & 32 & 504 & 120 & 1,283 \\
\hline 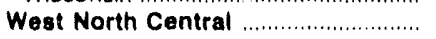 & 610 & 3,479 & 14 & 184 & 788 & 3,256 & 1,412 & 6,919 \\
\hline lowa & 158 & 332 & 0 & 0 & 159 & 8,36 & 317 & 1,168 \\
\hline 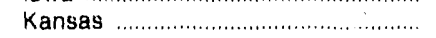 & 52 & 293 & 5 & 80 & 295 & 1,040 & 352 & 1,414 \\
\hline 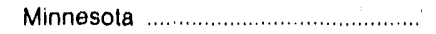 & 120 & 822 & 1 & 1 & 87 & 359 & 208 & 1,181 \\
\hline 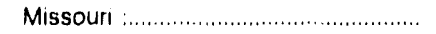 & 125 & 1,322 & 3 & 99 & 120 & 666 & 248 & 2,087 \\
\hline Nebraska & 91 & 375 & 5 & 5 & 113 & 313 & 209 & 693 \\
\hline 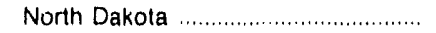 & 29 & 94 & 0 & 0 & 2 & 10 & 31 & 104 \\
\hline South Dakota & 35 & 241 & 0 & 0 & 12 & 32 & 47 & 273 \\
\hline South Atlantic & 269 & 5,545 & 13 & 448 & 196 & 4,295 & 478 & 10,287 \\
\hline Delaware & 15 & 110 & 2 & 187 & 0 & 0 & 17 & 297 \\
\hline District of Columbia ........................... & 2 & 256 & 0 & 0 & 0 & 0 & 2 & 256 \\
\hline 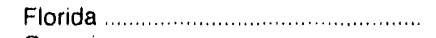 & 123 & 2,737 & 0 & 0 & 102 & 1,868 & 225 & 4,605 \\
\hline 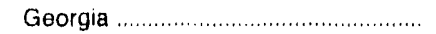 & 22 & 814 & 1 & 5 & 10 & 209 & 33 & 1,028 \\
\hline 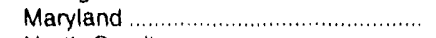 & 43 & 711 & 9 & 246 & 7 & 153 & 59 & 1,110 \\
\hline 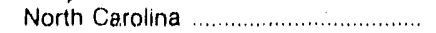 & 28 & 478 & 0 & 0 & 18 & 476 & 46 & 953 \\
\hline 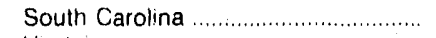 & 8 & 182 & 1 & 10 & 42 & 1,117 & 51 & 1,308 \\
\hline 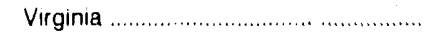 & 27 & 245 & 0 & 0 & 17 & 472 & 44 & 717 \\
\hline 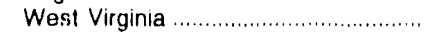 & 1 & 12 & 0 & 0 & 0 & 0 & 1 & 12 \\
\hline 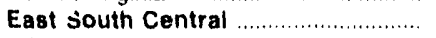 & 31 & 1.275 & 10 & 166 & 40 & 1,105 & 81 & 2,546 \\
\hline 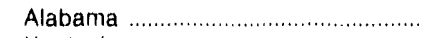 & 1 & 16 & 0 & 0 & 8 & 400 & 9 & 416 \\
\hline 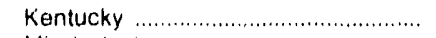 & 8 & 76 & 5 & 92 & 6 & 68 & 19 & 236 \\
\hline 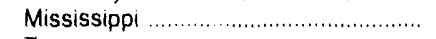 & 2 & 31 & 5 & 74 & 6 & 121 & 13 & 226 \\
\hline 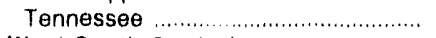 & 20 & 1,152 & 0 & 0 & 20 & 516 & 40 & 1,668 \\
\hline 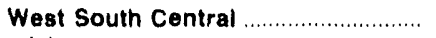 & 67 & 317 & 37 & 917 & 209 & 3,100 & 313 & 4,335 \\
\hline 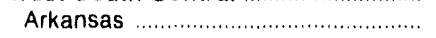 & 17 & 202 & 0 & 0 & 22 & 110 & 39 & 312 \\
\hline 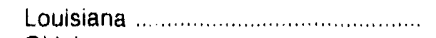 & 10 & 44 & 0 & 0 & 30 & 176 & 40 & 220 \\
\hline 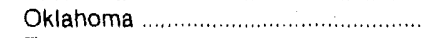 & 10 & 30 & 7 & 312 & 61 & 501 & 78 & 844 \\
\hline 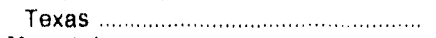 & 30 & 41 & 30 & 605 & 96 & 2,313 & 156 & 2,959 \\
\hline 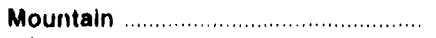 & 106 & 414 & 6 & 107 & 99 & 2,449 & 211 & 2,971 \\
\hline Arizona & 2 & 75 & 1 & 10 & 40 & 1,720 & 43 & 1,805 \\
\hline 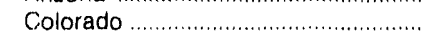 & 41 & 141 & 0 & 0 & 32 & 204 & 73 & 345 \\
\hline Idaho & 4 & 56 & 0 & 0 & 0 & 0 & 4 & 56 \\
\hline 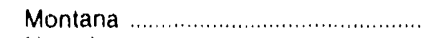 & 0 & 0 & 0 & 0 & 2 & 50 & 2 & 50 \\
\hline 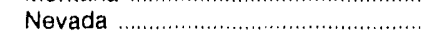 & 31 & 78 & 1 & 12 & 7 & 401 & 39 & 490 \\
\hline 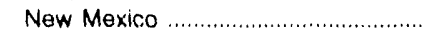 & 6 & 24 & 2 & 82 & 1 & 16 & 9 & 121 \\
\hline Utah & 13 & 24 & 2 & 3 & 17 & 59 & 32 & 86 \\
\hline 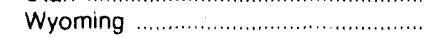 & 9 & 18 & 0 & 0 & 0 & 0 & 9 & 18 \\
\hline 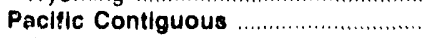 & 50 & 642 & 0 & 0 & 67 & 3,232 & 117 & 3,874 \\
\hline California & 40 & 548 & 0 & 0 & 52. & 2,195 & 92 & 2,743 \\
\hline Oregon & 2 & 6 & 0 & 0 & 8 & 448 & 10 & 454 \\
\hline Washington & 8 & 88 & 0 & 0 & 7 & 590 & 15 & 678 \\
\hline 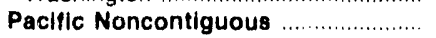 & 394 & 789 & 7 & 281 & 20 & 428 & 421 & 1,498 \\
\hline 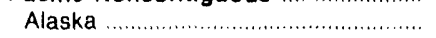 & 346 & 464 & 7 & 281 & 20 & 428 & 373 & 1,173 \\
\hline 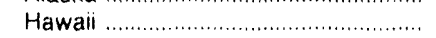 & 48 & 325 & 0 & 0 & 0 & 0 & 48 & 325 \\
\hline U.S. Total & 2,365 & 22,351 & 126 & 2,917 & 1,835 & 24,745 & 4,326 & 50,012 \\
\hline
\end{tabular}

1 Net summer capability.

Note: Totals may not equal sum of components because of independent rounding.

Source: Energy Information Administration, Form ElA-860, "Annual Electric Generator Report." 
Table 10. Planned Capability Aaditions by Energy Source and North American Electric Reliability Council Region, Alaska, and Hawail, 1990-1999

\begin{tabular}{|c|c|c|c|c|c|c|}
\hline \multirow{2}{*}{$\begin{array}{l}\text { North American Electric } \\
\text { Reliability Councll Reglon, } \\
\text { Alaska, and Hawail }\end{array}$} & \multicolumn{2}{|c|}{ Total } & \multicolumn{2}{|c|}{ Petroleum \& Gas } & \multicolumn{2}{|c|}{ Coal } \\
\hline & $\begin{array}{l}\text { Number of } \\
\text { Generators }\end{array}$ & $\begin{array}{l}\text { Capabllity' } \\
\text { (megawatts) }\end{array}$ & $\begin{array}{l}\text { Number of } \\
\text { Generators }\end{array}$ & $\begin{array}{l}\text { Capabillty' } \\
\text { (megawatts) }\end{array}$ & $\begin{array}{l}\text { Number of } \\
\text { Generators }\end{array}$ & $\begin{array}{l}\text { Capabillty' } \\
\text { (megawatts) }\end{array}$ \\
\hline 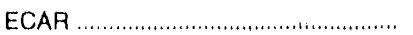 & 36 & 4,248 & 29 & 2,363 & 4 & 1,810 \\
\hline 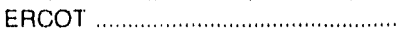 & 28 & 9,221 & 12 & 1,413 & 12 & 5,504 \\
\hline MAAC & 33 & 3,678 & 29 & 2,752 & 2 & 790 \\
\hline 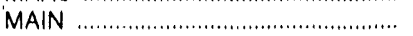 & 26 & 1,359 & 22 & 1,291 & 1 & 60 \\
\hline MAPP(U.S.) & 18 & 1.438 & 15 & 614 & 2 & 823 \\
\hline NPCC(U.S.) & 28 & 1,415 & 1 & 30 & 0 & 0 \\
\hline SERC & 87 & 12,654 & 56 & 4,387 & 8 & 2,906 \\
\hline SPP & 34 & 1,916 & 29 & 1,332 & 2 & 560 \\
\hline WSCC(US.) & 74 & 5,302 & 14 & 715 & 8 & 3,370 \\
\hline Contiguous U.S. & 364 & 41,230 & 207 & 14,898 & 39 & 15,813 \\
\hline Alaska & 4 & 2 & 1 & 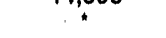 & 0 & 0 \\
\hline 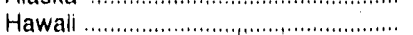 & 0 & 0 & 0 & 0 & 0 & 0 \\
\hline \multirow[t]{3}{*}{ 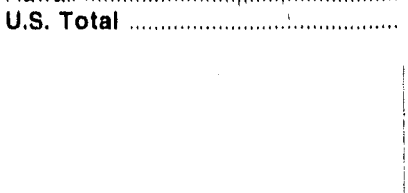 } & 368 & 41,232 & 208 & 14,899 & 39 & 15,813 \\
\hline & \multicolumn{2}{|c|}{ Nuclear } & \multicolumn{2}{|c|}{ Hydroelectric } & \multicolumn{2}{|c|}{ Other ${ }^{2}$} \\
\hline & $\begin{array}{l}\text { Number of } \\
\text { Generators }\end{array}$ & $\begin{array}{l}\text { Capability' } \\
\text { (megawatts) }\end{array}$ & $\begin{array}{l}\text { Number of } \\
\text { Generators }\end{array}$ & $\begin{array}{l}\text { Capability' } \\
\text { (megawatts) }\end{array}$ & $\begin{array}{l}\text { Number of } \\
\text { Generators }\end{array}$ & $\begin{array}{l}\text { Capabillty' } \\
\text { (megawatts) }\end{array}$ \\
\hline 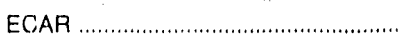 & 0 & 0 & 3 & 75 & 0 & 0 \\
\hline ERCOT & 2 & 2,300 & 2 & 4 & 0 & 0 \\
\hline MAAC & 0 & 0 & 0 & 0 & 2 & 136 \\
\hline MAIN & 0 & 0 & 3 & 8 & 0 & 0 \\
\hline MAPP(U.S.) & 0 & 0 & 1 & $\cdot$ & 0 & 0 \\
\hline NPCC(U.S.) & 1 & 1,150 & 26 & 234 & 0 & 0 \\
\hline SERC & 2 & 2,340 & 12 & 2,271 & 9 & 749 \\
\hline SPP & 0 & 0 & 3 & 34 & 0 & 0 \\
\hline WSCC(U.S.) & 0 & 0 & 47 & 875 & 5 & 342 \\
\hline Contiguous U.S. & 5 & 5,790 & 97 & 3,501 & 16 & 1,227 \\
\hline Alaska & 0 & $\begin{array}{r}0 \\
0\end{array}$ & 3 & 1 & 0 & $\quad 0$ \\
\hline 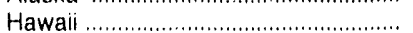 & 0 & 0 & 0 & 0 & 0 & 0 \\
\hline U.S. Total & 5 & 5,790 & 100 & 3,502 & 16 & 1,227 \\
\hline
\end{tabular}

Net summer capability.

2 Includes waste heat and solar.

- Value less than 0.5 .

Note: - Totals may not equal sum of components because of independent rounding.

Source: Energy Information Administration, Form ElA-860, "Annual Electric Generator Report." 
Table 11. Planned Capability Additions by Energy Source, Census Division, and State, 1990-1999

\begin{tabular}{|c|c|c|c|c|c|c|}
\hline \multirow{2}{*}{$\begin{array}{l}\text { Census Division } \\
\text { and State }\end{array}$} & \multicolumn{2}{|c|}{ Total } & \multicolumn{2}{|c|}{ Petroleum \& Gas } & \multicolumn{2}{|c|}{ Coal } \\
\hline & $\begin{array}{l}\text { Number of } \\
\text { Generators }\end{array}$ & $\begin{array}{l}\text { Capability' } \\
\text { (megawatts) }\end{array}$ & $\begin{array}{l}\text { Number of } \\
\text { Generators }\end{array}$ & $\begin{array}{l}\text { Capabillty' } \\
\text { (megawatts) }\end{array}$ & $\begin{array}{l}\text { Number of } \\
\text { Generators }\end{array}$ & $\begin{array}{l}\text { Capabllity' } \\
\text { (megawatts) }\end{array}$ \\
\hline 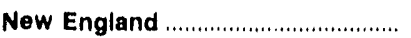 & 12 & 1,260 & 1 & 30 & 0 & 0 \\
\hline 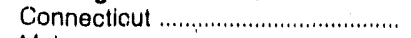 & 0 & 0 & 0 & 0 & 0 & 0 \\
\hline 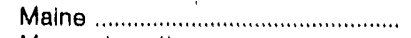 & 7 & 73 & 0 & 0 & 0 & 0 \\
\hline 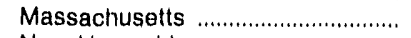 & 1 & 30 & 1 & 30 & 0 & 0 \\
\hline New Hampshire .................................... & 1 & 1,150 & 0 & 0 & 0 & 0 \\
\hline 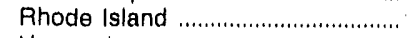 & 0 & 0 & 0 & 0 & 0 & 0 \\
\hline 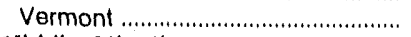 & 3 & 7 & 0 & 0 & 0 & 0 \\
\hline Middle Atlantic .................................. & 28 & 1,452 & 11 & 1,284 & 0 & 0 \\
\hline 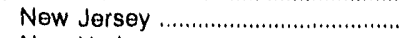 & 12 & 1,208 & 11 & 1,284 & 0 & 0 \\
\hline 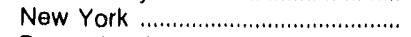 & 16 & 154 & 0 & 0 & 0 & 0 \\
\hline 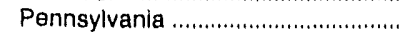 & 0 & 0 & 0 & 0 & 0 & 0 \\
\hline East North Central ............................ & 49 & 4,174 & 42 & 2,776 & 4 & 1,390 \\
\hline 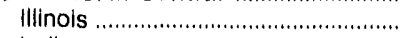 & 2 & 71 & 2 & 71 & 0 & 0 \\
\hline 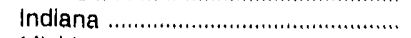 & 10 & 914 & 10 & 914 & 0 & 0 \\
\hline 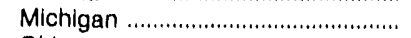 & 0 & 0 & 0 & 0 & ) & 0 \\
\hline 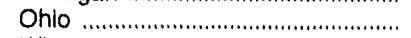 & 16 & 2,085 & 13 & 755 & 3 & 1,330 \\
\hline 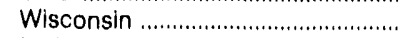 & 21 & 1,103 & 17 & 1,035 & 1 & 60 \\
\hline 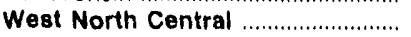 & 47 & 3,176 & 42 & 1,803 & 4 & 1,373 \\
\hline lowa & 10 & 241 & 9 & 241 & 0 & 0 \\
\hline 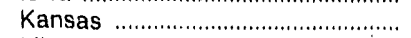 & 7 & 18 & 7 & 18 & 0 & 0 \\
\hline 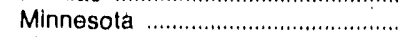 & 3 & 563 & 2 & 163 & 1 & 400 \\
\hline 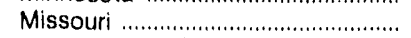 & 21 & 1,687 & 19 & 1,137 & 2 & 550 \\
\hline 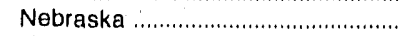 & 2 & 173 & 2 & 173 & 0 & 0 \\
\hline 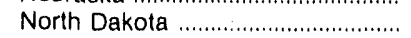 & 1 & 423 & 0 & 0 & 1 & 423 \\
\hline 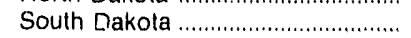 & 3 & 70 & 3 & 70 & 0 & 0 \\
\hline 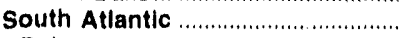 & 94 & 11,253 & 66 & 5,275 & 8 & 2,904 \\
\hline 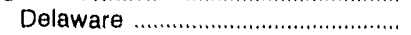 & 2 & 204 & 1 & 83 & 0 & 0 \\
\hline District of Columbia & 0 & 0 & 0 & 0 & 0 & 0 \\
\hline Florida & 43 & 3,955 & 36 & 2,937 & 2 & 458 \\
\hline 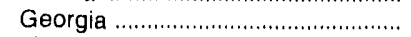 & 7 & 1,176 & 0 & 0 & 0 & 0 \\
\hline 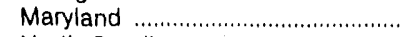 & 19 & 2.176 & 17 & 1,386 & 2 & 790 \\
\hline North Carolina .................................... & 0 & 0 & 0 & 0 & 0 & 0 \\
\hline 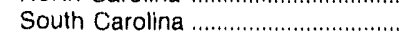 & 11 & 2,300 & 4 & 335 & 2 & 870 \\
\hline Virginia & 12 & 1,442 & 8 & 536 & 2 & 786 \\
\hline 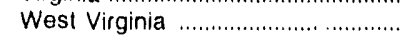 & 0 & 0 & 0 & 0 & 0 & 0 \\
\hline East South Central & 24 & 5,029 & 14 & 1,273 & 3 & 1,272 \\
\hline Alabama & 8 & 1,239 & 6 & 447 & 2 & 792 \\
\hline 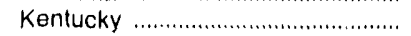 & 10 & 1,248 & 6 & 693 & 1 & 480 \\
\hline 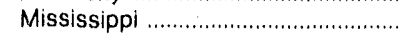 & 4 & 202 & 2 & 133 & 0 & 0 \\
\hline Tennessee & 2 & 2,340 & $\overline{0}$ & 0 & 0 & 0 \\
\hline 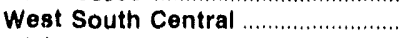 & 39 & 9,732 & 20 & 1,891 & 12 & 5,504 \\
\hline 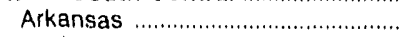 & 4 & 116 & 1 & 83 & 0 & 0 \\
\hline 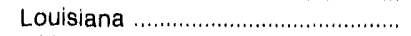 & 0 & 0 & 0 & 0 & 0 & 0 \\
\hline 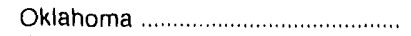 & 4 & 263 & 4 & 263 & 0 & 0 \\
\hline 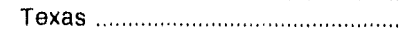 & 31 & 9,353 & 15 & 1,545 & 12 & 5,504 \\
\hline 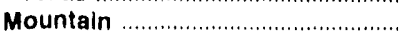 & 42 & 4,506 & 11 & 566 & 8 & 3,370 \\
\hline 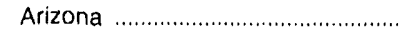 & 10 & 1,139 & 4 & 247 & 2 & 720 \\
\hline 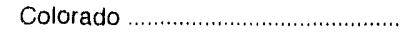 & 2 & 153 & 1 & 62 & 0 & 0 \\
\hline 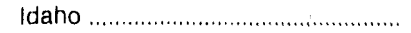 & 7 & 143 & 0 & 0 & 0 & 0 \\
\hline 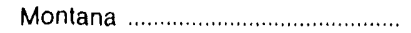 & 0 & 0 & 0 & 0 & 0 & 0 \\
\hline 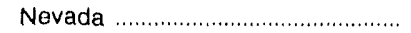 & 11 & 2,655 & 4 & 256 & 5 & 2,250 \\
\hline 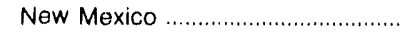 & 0 & 0 & 0 & 0 & 0 & C \\
\hline Utah & 12 & 415 & 2 & 2 & ; & 400 \\
\hline 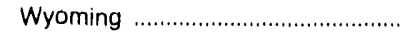 & 0 & 0 & 0 & 0 & 0 & 0 \\
\hline Pacific Contiguous & 39 & 647 & 0 & 0 & 0 & 0 \\
\hline 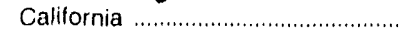 & 23 & 597 & 0 & 0 & 0 & 0 \\
\hline 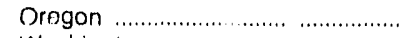 & 0 & 0 & 0 & 0 & 0 & 0 \\
\hline 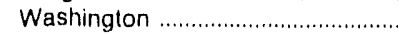 & 6 & 50 & 0 & 0 & 0 & 0 \\
\hline Pacific Noncontiguous ................... & 4 & 2 & 1 & $*$ & 0 & 0 \\
\hline 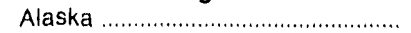 & 4 & 2 & 1 & • & 0 & 0 \\
\hline 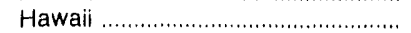 & 0 & 0 & 0 & 0 & 0 & 0 \\
\hline 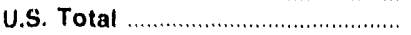 & 368 & 41,232 & 208 & 14,899 & 39 & 15,813 \\
\hline
\end{tabular}

- Net summer capability

- Value less than 0.5 .

Note: - Totals may not equal sum of comporients because of independent rounding.

Source. Energy Information Administration, Form ElA-860, "Annual Electric Generator Report." 
Table 11. Planned Capability Additions by Energy Source, Census Division, and State, 1990-1999 (Continued)

\begin{tabular}{|c|c|c|c|c|c|c|}
\hline \multirow[b]{2}{*}{$\begin{array}{l}\text { Census Division } \\
\text { and State }\end{array}$} & \multicolumn{2}{|c|}{ Nuclear } & \multicolumn{2}{|c|}{ Hydroelectric } & \multicolumn{2}{|c|}{ Other' } \\
\hline & $\begin{array}{l}\text { Number of } \\
\text { Generators }\end{array}$ & $\begin{array}{l}\text { Capability }{ }^{2} \\
\text { (megawatts) }\end{array}$ & $\begin{array}{l}\text { Number of } \\
\text { Generators }\end{array}$ & $\begin{array}{l}\text { Capabillty } \\
\text { (megawatts) }\end{array}$ & $\begin{array}{l}\text { Number of } \\
\text { Generators }\end{array}$ & $\begin{array}{l}\text { Capability } \\
\text { (megawatts) }\end{array}$ \\
\hline 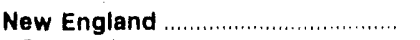 & $\uparrow$ & 1,150 & 10 & 80 & 0 & 0 \\
\hline 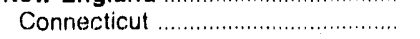 & 0 & 0 & 0 & 0 & 0 & 0 \\
\hline Maine ............................................... & 0 & 0 & 7 & 73 & 0 & 0 \\
\hline 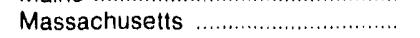 & 0 & 0 & 0 & 0 & 0 & 0 \\
\hline New Hamyshire ............................. & 1 & 1,150 & 0 & 0 & 0 & 0 \\
\hline 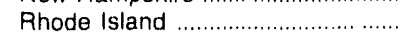 & 0 & 0 & 0 & 0 & 0 & 0 \\
\hline Vermont & 0 & 0 & 3 & 7 & 0 & 0 \\
\hline 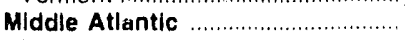 & 0 & 0 & 16 & 154 & 1 & 14 \\
\hline 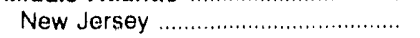 & 0 & 0 & 0 & 0 & 1 & 14 \\
\hline 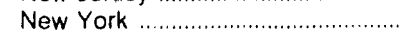 & 0 & 0 & 16 & 154 & 0 & 0 \\
\hline 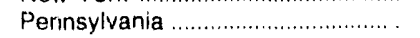 & 0 & 0 & 0 & 0 & 0 & 0 \\
\hline 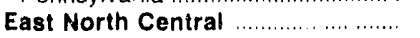 & 0 & 0 & 3 & 8 & 0 & 0 \\
\hline 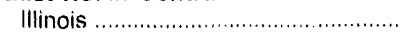 & 0 & 0 & 0 & 0 & 0 & 0 \\
\hline 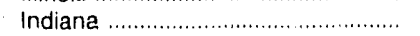 & 0 & 0 & 0 & 0 & 0 & 0 \\
\hline 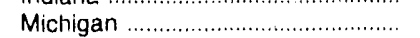 & 0 & 0 & 0 & 0 & c & 0 \\
\hline Ohio & 0 & 0 & 0 & 0 & 0 & 0 \\
\hline 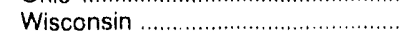 & 0 & 0 & 3 & 8 & 0 & 0 \\
\hline 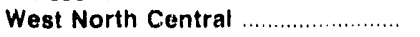 & 0 & 0 & 1 & $*$ & 0 & 0 \\
\hline lowa & 0 & 0 & 1 & $\star$ & 0 & 0 \\
\hline 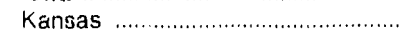 & 0 & 0 & 0 & 0 & 0 & 0 \\
\hline 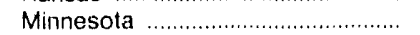 & 0 & 0 & 0 & 0 & 0 & 0 \\
\hline 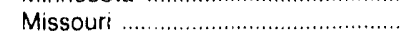 & 0 & 0 & 0 & 0 & 0 & 0 \\
\hline Nebraska & 0 & 0 & 0 & 0 & 0 & 0 \\
\hline North Dakota & 0 & 0 & 0 & 0 & 0 & 0 \\
\hline 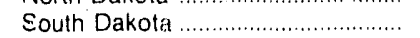 & 0 & 0 & 0 & 0 & 0 & 0 \\
\hline 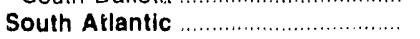 & 0 & 0 & 12 & 2,271 & 8 & 802 \\
\hline Delaware & 0 & 0 & 0 & 0 & 1 & 122 \\
\hline District of Columbia ......................... & 0 & 0 & 0 & 0 & 0 & 0 \\
\hline Florida & 0 & 0 & 0 & 0 & 5 & 560 \\
\hline Georgia & 0 & 0 & 7 & 1,176 & 0 & 0 \\
\hline Maryland & 0 & 0 & 0 & 0 & 0 & 0 \\
\hline North Carolina & 0 & 0 & 0 & 0 & 0 & 0 \\
\hline 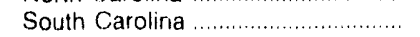 & 0 & 0 & 5 & 1,095 & 0 & 0 \\
\hline Virginia & 0 & 0 & 0 & 0 & 2 & 120 \\
\hline West Virginia & 0 & 0 & 0 & 0 & 0 & 0 \\
\hline 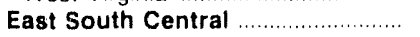 & 2 & 2,340 & 3 & 75 & 2 & 69 \\
\hline Alabama & 0 & 0 & 0 & 0 & 0 & 0 \\
\hline 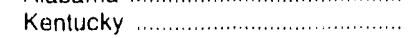 & 0 & 0 & 3 & 75 & 0 & 0 \\
\hline 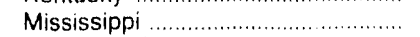 & 0 & 0 & 0 & 0 & 2 & 69 \\
\hline 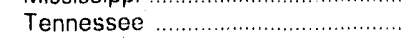 & 2 & 2,340 & 0 & 0 & 0 & 0 \\
\hline 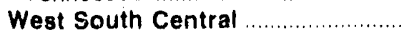 & 2 & 2,300 & 5 & 37 & 0 & 0 \\
\hline Arkansas & 0 & 0 & 3 & 34 & 0 & 0 \\
\hline 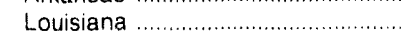 & 0 & 0 & 0 & 0 & 0 & 0 \\
\hline Oklahoma & 0 & 0 & 0 & 0 & 0 & 0 \\
\hline 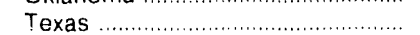 & 2 & 2,300 & 2 & 4 & 0 & 0 \\
\hline Mountain & 0 & 0 & 21 & 421 & 2 & 149 \\
\hline 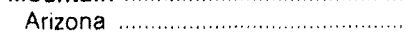 & 0 & 0 & 4 & 172 & 0 & 0 \\
\hline 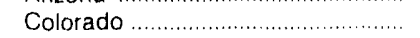 & 0 & 0 & 1 & 92 & 0 & 0 \\
\hline Idaho & 0 & 0 & 7 & 143 & 0 & 0 \\
\hline 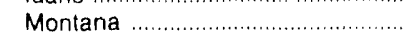 & 0 & 0 & 0 & 0 & 0 & 0 \\
\hline Nevada & 0 & 0 & 0 & 0 & 2 & 149 \\
\hline New Mexico & 0 & 0 & 0 & 0 & 0 & 0 \\
\hline Utah & 0 & 0 & 9 & 13 & 0 & 0 \\
\hline 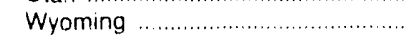 & 0 & 0 & 0 & 0 & 0 & 0 \\
\hline Pacific Contiguous & 0 & 0 & 26 & 454 & 3 & 193 \\
\hline California & 0 & 0 & 20 & 404 & 3 & 193 \\
\hline Oregon & 0 & 0 & 0 & 0 & 0 & 0 \\
\hline Washington & 0 & 0 & 6 & 50 & 0 & 0 \\
\hline Pacific Noncontiguous & 0 & 0 & 3 & 1 & 0 & 0 \\
\hline Alaska & 0 & 0 & 3 & 1 & 0 & 0 \\
\hline 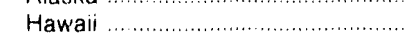 & 0 & 0 & j & 0 & 0 & 0 \\
\hline U.S. Total & 5 & 5,790 & 100 & 3,502 & 16 & 1,227 \\
\hline
\end{tabular}

Includes waste heal and solar

- Net summer capability.

- Value less than 0.5

Note: Totals may not equal sum of components because of independent rounding.

Source: Energy Information Administration, Form EIA-860 "Annual Electric Generator Report." 


\section{Net Generation}

This chapter provides summary statistics on the amount of electricity produced in the United States. The different energy sources used to produce electric power are also discussed in this chapter.

\section{Background}

The production of electricity is generally referred to as generation, and is measured in kilowatthours. Gross generation is the amount of power produced by an electric power plant (station), measured at the terminals of the plant (that is, prior to the point at which the power leaves the station and is available to the system). Some of the electric power generated at a power plant is used to operate equipment at the plant. Power used at the plant generally ranges between 1 percent (for hydroelectric units) and 7 percent (for steam-electric units). Net generation is the power available to the system (gross generation less use at the plant); however, it is greater than that available to consumers due to losses during transmission and distribution. Net generation is the measure of electric power production used in this report.

The energy sources used for producing electricity fall into two broad categories: nonrenewable (those that cannot be replaced once used) and renewable (those that are constantly replenished). The most common nonrenewable energy sources used for generation are the fossil fuels (coal, petroleum, and gas) and uranium. These energy sources account for about 90 percent of U.S. net generation. The renewable energy sources include the water in rivers, streams, or lakes (hydroelectric); the light or heat of the sun (solar); wind; heat from beneath the surface of the earth (geothermal); and organic wastes, which are produced by either natural or technological processes.

The choice of generating technology and fuel used to produce electricity is of major importance to electric utilities because the goal is to provide the least-cost and most reliable electricity possible to consumers. A variety of factors influence the selection of technoiogy and fuel including the capital costs of the technology, operations and maintenance costs, environmental restrictions on the technology and use of the fuel, regu. lations affecting fuel use, the cost and availability of the fuel, and the availability of capacity associated with the type of fuel. The previous chapter covers the type and amount of U.S. generating capability cur- rently existing, as well as, proposed capability additions planned for the period 1990 through 1999. The succeeding chapter covers the costs of fossil fuels.

\section{Generation from Fossil Fuels}

Coal. Historically, most of the generation in the United States has been from coal. After the Arab oil embargo of 1973, concerns over the availability of petroleum imports, increasing petroleum costs, and curtailments of natural gas made coal-fired generation even more important. In 1978, the passage of the Powerplant and Industrial Fuel Use and Natural Gas Policy Acts encouraged further use of coal by electric utilities. Although both Federal and State environmental laws and regulations exist, during the 1970 's, renewed interest in environmental issues raised concerns about electric power plant emissions, particularly from those burning coal. The Clean Air Act Amendments of 1990, a bill whose goal is a 10-million-ton reduction in sulfur dioxide emissions and a 2 -million-ton reduction in nitrogen oxide emissions from 1980 levels, was signed into law by President Bush on November 15, 1990 (see the chapter on Environmental Statistics). Coal-fired generation continues to provide more than one-half the total net generation of electricity in the Nation. Most of the electricity production from coal occurs in the East North Central and South Atlantic Census Divisions where substantial amounts of coal are mined.

Petroleum. During the early 1970 's, electric utilities used petroleum extensively to generate electricity because it was a relatively inexpensive fuel. However, after the 1973 embargo by the Organization of Petroleum Exporting Countries (OPEC) on petroleum exports 10 the United States, petroleum prices rose sharply. Further price increases occurred in 1979 and 1980 following the Iranian revolution and subsequent reductions in Iranian petroleum exports. Consequently during the past decade, utilities have not built large, petroleum-fired steam units. In addition, many utilities have either converted steam units to coal or switched fuels where dual-fired capability exists. Most of the utilities that still rely heavily on petroleum to generate electricity are located along the eastern seaboard and in California.

Gas. The demand for gas to heat homes and serve business and industry has historically taken priority 
over the demand of electric utilities under both Federal and State regulations. In the 1970's, many utilities were on occasion denied gas when available pipelines reached capacity in serving heating demand during the months from November to March (the peak heating seatson). By the middle 1970's, curtailments to electric utilities also occasionally occurred during the nonheating season as producers conserved supply in preparation for heating season demand. In the face of an attractive interstate price structure but deprived of supplies during many months of the year, utilities in the 1970's used relatively less-expensive gas when it was available, then switched to other more expensive fuels when gas supplies were curtailed. Gas became more available to utilities with the passage of the NatLisal Gas Policy Act of 1978 and more frequent exemptions from the gas-use restrictions of the Powerplant and Industrial Fuel Use Act (Fuel Use Act) of 1978. Amendments to the Fuel Use Act in 1987 have created potential for additional use of gas. These amendments eased restrictions on the use of gas by removing a legal requirement to obtain an exemption for the construction of new gas-fired generating capability. The West South Central Censtis Division, where most of the gas production in the Nation occurs, supplies more than half of the gas-fired generation in the country.

\section{Generation from Uranium}

Generation from nuclear power has generally increased since the 1950's, and this trend centinues. Since 1984. nuclear plants have provided the second largest share of fotal U.S. generation of electricity, after coalfired plants. Although ao new nuclear units have been ordered since 1978, and the units ordered after 1974 were not huilt, many that were under construction have either been completed and entered service or will enter service in the near future. Licensing delays, questions about radioactive waste disposal, and concern about maclear plant salety have slowed these units from entering service and are still major obstacles to additional growith in the use of this energy source for generating electricity. Most of the nuclear-powered generation comes from the Middle Atlantic, East North Central. and South Atlantic Census Divisions (where over 60 percent of the nuclear units in the country are located.)

\section{Generation from Renewable Fuels}

Hydroelectric. Water is currently the leading renew able encegy source used ro generate electric power. Hydroelectric plants operate where suitable waterWays are avalable: many of the hest of these sites have atready been develeped. Generating electricily using waler has severai advantages. The major advantage is that water is a resource, which is renewable. A pumped-storage hydroelectric plant offers a second advantage in that these systems use electricity produced by other generators (generally those that serve baseload requirements) to pump water from one storage area (reservoir) to another. Later this water is allowed to flow from the higher reservoir to the lowr one through the generator. In this manner, the same energy source (water) is used twice. Net hydroelectric generation statistics reflected in this report are derived by deducting the generation used for pumping (in house use) from the total (gross) hydroelectric generation. Since water is renewable, it is a source of cheap power. In addition, because there is no fuel combustion, there is little air pollution in comparison with fossil fuel plants and limited thermal pollution in comparison with nuclear plants. Like other energy sources, the use of water for generation has limitations, including environmental impacts caused by damming rivers and streams, which affects the habitats of the local plant, fish, and animal life. Over 60 percent of the hydroelectric power in the United States is generated in the Pacific and Rocky Mountain States.

Other Sources. Other renewable resources geothermal (heat energy deep beneath the surface of the earth), wood, waste, wind, and the sun (solar) -are energy sources that are constantly replenished. These energy sources have received increased attention in recent years, but a limited number of such generating facilities are in use today. The major obstacles to their use are technology and cost. Currently, renewable resources (other than water) supply less than 1 percent of the electricity for the Nation. Most of the electricity produced from this category is from geothermal power. Electric utilities currently operate geothermal plants in three States (California, Hawaii, and Utah). The Gej'sers, operated by the Pacific Gas and Electric Company, is the largest geothermal plant in the Nation. Only a few utilities operate units that produce electricity from wind and solar energy. The largest share of the electricity from these energy sources is produced from facilities in California. Wood and waste resources can be used to replace fossil fuels in utility boilers. To date, just a few electric generating units have been built that use wood or waste products as a primary fuel.

\section{Data Sources}

The data in the following tables are aggregated at the mational, regional, and State levels for the period 1985 through 1989. Data in the lables were obtained from the Form FIA -759, "Monthly Power Plant Report." The Form I:IA-759 is used to collect monthly data from all operators of electric utilities (approximately $x(x)$ ) in the United States. Moredetailed statistics from the Form EAA-759 are published in the Electric Power nomlhly 
Table 12. Net Generation by Energy Source, 1985-1989 (Million Kilowatthours)

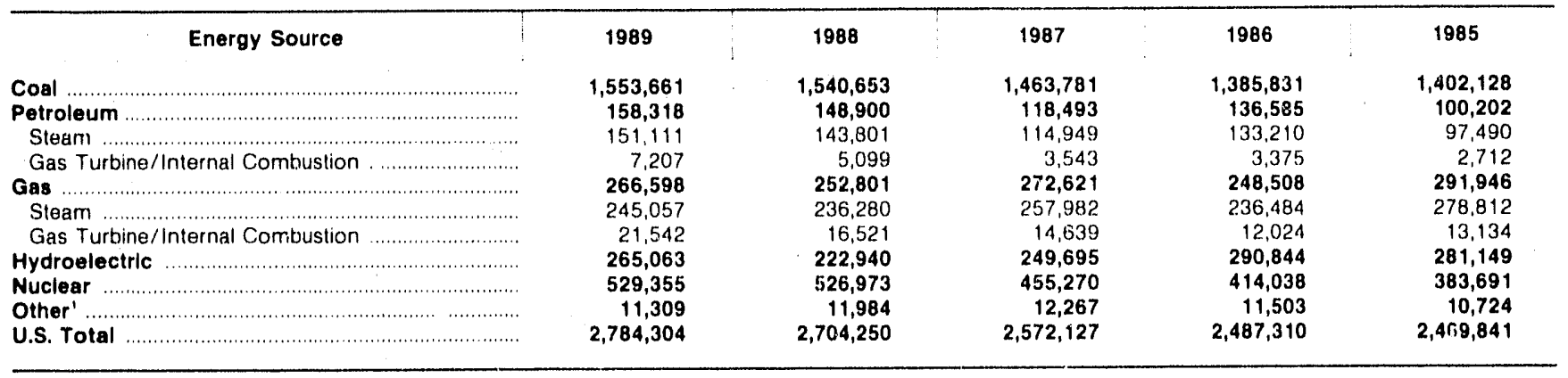

1 Includes geothermal, wood, wind, waste, and solar.

Note: Totals riay not equlal sum of components because of independent rounding.

Source: Energy Information Administration, Form ElA.759. "Monthly Power Plant Report."

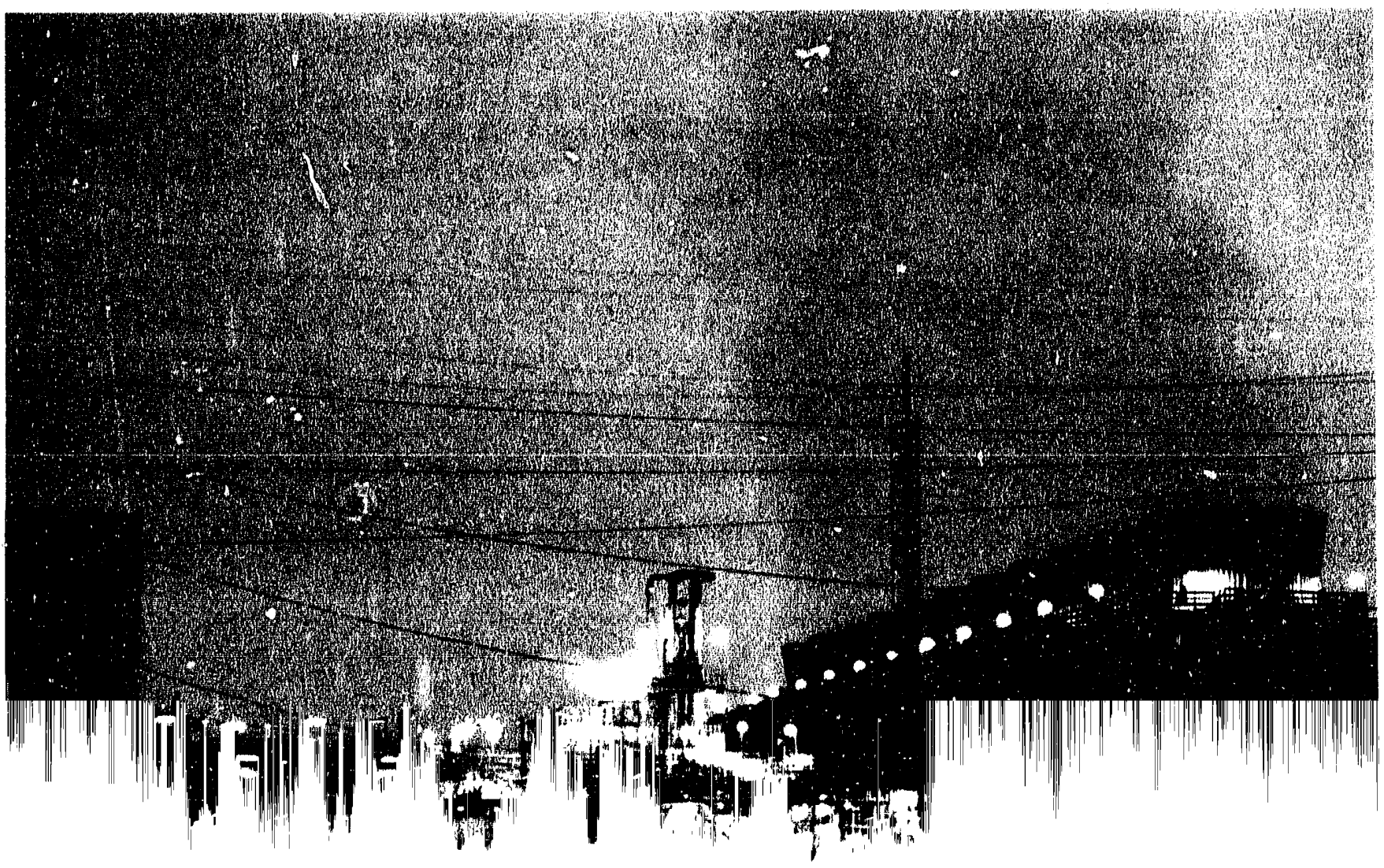


Table 13. Net Generation by Selected Prime Mover, Census Division, and State, 1989 and 1988 (Million Kilowatthours)

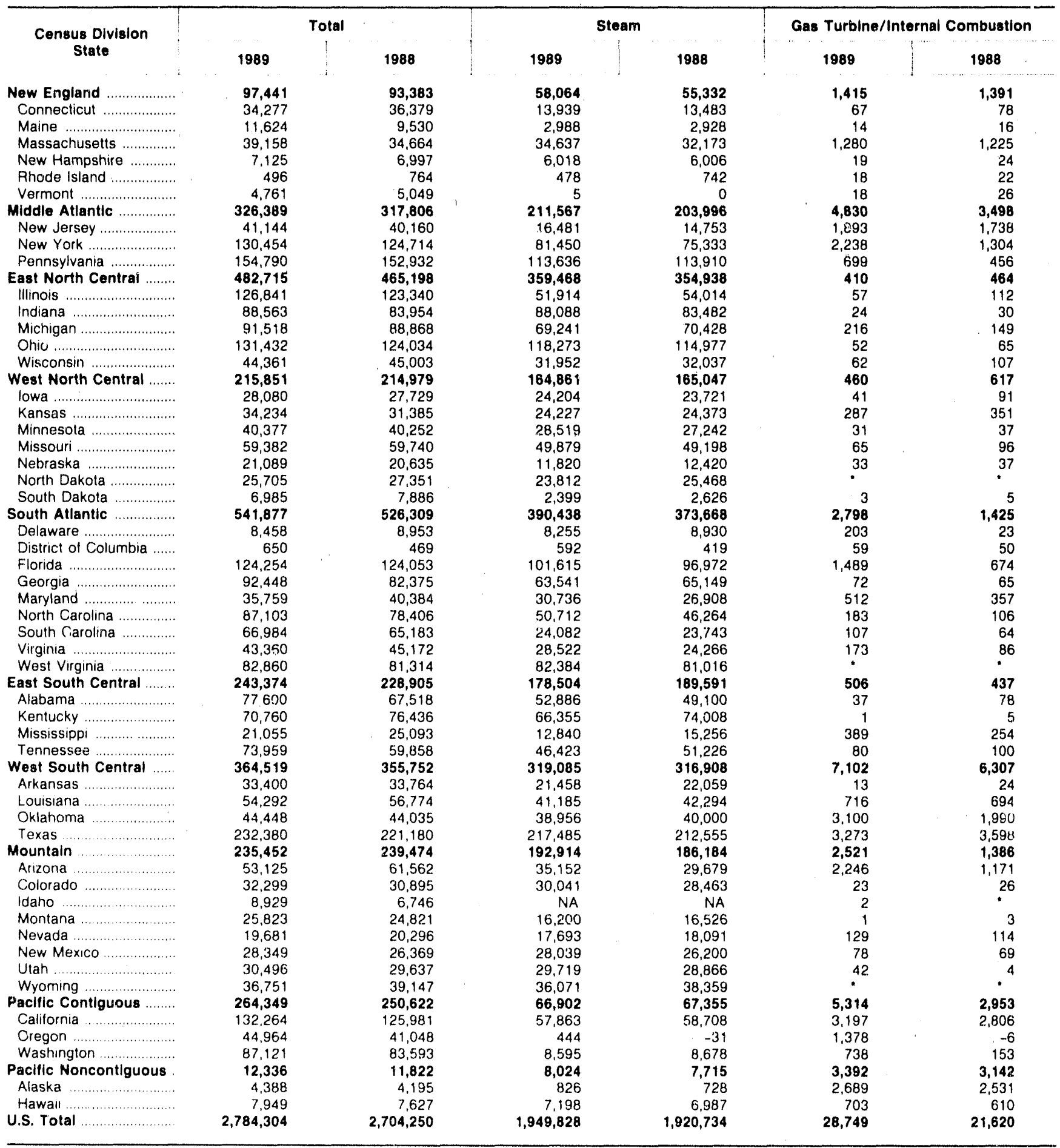

- Value less than 0.5 million kilowatthours

NA : Not applicable

Notes: - Negative generation denotes that electric power consumed for plant use exceeds gross generaticn. $\bullet$ Totals may not equal sum of components because of independent rounding.

Source: Energy Information Administration, Form ElA.759, "Monthly Power Plant Repcrt." 
Table 14. Net Generation by Energy Source, Census Division, and State, 1989 and 1988 (Million Kilowatthours)

\begin{tabular}{|c|c|c|c|c|c|c|}
\hline \multirow{2}{*}{$\begin{array}{c}\text { Census Division } \\
\text { State }\end{array}$} & \multicolumn{2}{|c|}{ Coal } & \multicolumn{2}{|c|}{ Petroleum } & \multicolumn{2}{|c|}{ Gas } \\
\hline & 1989 & 1988 & 1989 & 19R9 & 1989 & 1988 \\
\hline New England ..................... & 17,248 & 16,979 & 37,238 & 37,811 & 5,193 & 1,933 \\
\hline Connecticut ...................... & 2,095 & 2,094 & 11,575 & 11,356 & 336 & 111 \\
\hline 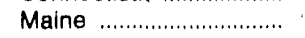 & NA & NA & 3,002 & 2,944 & NA. & NA \\
\hline 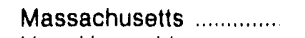 & 12,088 & 11,687 & 19,139 & 19,908 & 4,690 & 1,802 \\
\hline New Hampshire ............ & 3,065 & 3,197 & 2,970 & 2,828 & 1 & 5 \\
\hline 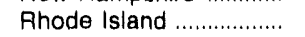 & 0 & 0 & 333 & 749 & 163 & . $\quad 15$ \\
\hline Vermont & NA & NA & 18 & 26 & 4 & NA \\
\hline Middle Atlantlc ................... & 139,530 & 136,163 & 54,782 & 52,832 & 22,085 & 18,499 \\
\hline 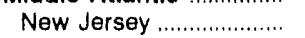 & 8,392 & 7,163 & 5,351 & 5,052 & 4,631 & 4,276 \\
\hline New York ......................... & 25,224 & 22,761 & 41,323 & 39,865 & 17,141 & 14,012 \\
\hline Pennsylvania .................... & 105,913 & 106,239 & 8,109 & 7,915 & 312 & 211 \\
\hline East North Central ........ & 355,688 & 350,816 & 2,666 & 3,006 & 1,524 & 1,580 \\
\hline Illinois ............................. & 51,124 & 52,994 & 339 & 697 & 508 & 435 \\
\hline Indiana ......................... & 87,330 & 82,813 & 409 & 383 & 374 & 317 \\
\hline Michigan .......................... & 67,618 & 68,578 & 1,407 & 1,414 & 432 & 585 \\
\hline 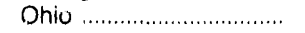 & 117,819 & 114,564 & 442 & 415 & 64 & 63 \\
\hline 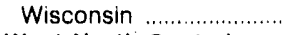 & 31,796 & 31,867 & 70 & 97 & 147 & 180 \\
\hline West North Central ....... & $162,2.78$ & 162,491 & 774 & 571 & 2,269 & 2,602 \\
\hline lowa & 24,041 & 23,345 & 43 & 49 & 161 & 417 \\
\hline 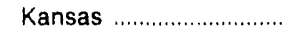 & 22,907 & 23,089 & 109 & 147 & 1,498 & 1,489 \\
\hline 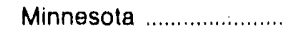 & 27,839 & 26,726 & 405 & 142 & 306 & 410 \\
\hline Missouri ............................ & 49,748 & 49,051 & 114 & 131 & 82 & 112 \\
\hline Nebraska ......................... & 11,582 & 12,225 & 57 & 69 & 215 & 163 \\
\hline North Dakota ................... & 23,774 & 25,450 & 38 & 18 & $\star$ & $\star$ \\
\hline 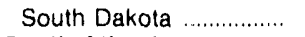 & 2.387 & 2,605 & 9 & 15 & 6 & 11 \\
\hline South Atlantic & 332,004 & 323,188 & 40,588 & 35,930 & 20,645 & 15,975 \\
\hline 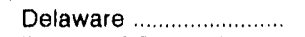 & 5,066 & 5,788 & 2,566 & 2,848 & 826 & 317 \\
\hline District of Columbia ...... & NA & NA & 650 & 469 & NA & NA \\
\hline 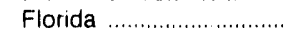 & 59,418 & 57,516 & 26,280 & 25,387 & 17,407 & 14,744 \\
\hline 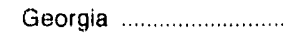 & 63,405 & 64,834 & 158 & 260 & 50 & 119 \\
\hline 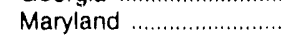 & 23,627 & 23,316 & 6,044 & 3,547 & 1,577 & 402 \\
\hline North Carolina ................ & 50,524 & 46,090 & $\quad 256$ & 225 & 115 & 55 \\
\hline South Carolina .............. & 23,800 & 23,485 & 134 & 96 & 255 & 226 \\
\hline Virginia & 24.059 & 21,413 & 4.237 & 2,838 & 399 & 102 \\
\hline 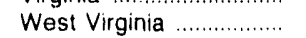 & 82,105 & 80,747 & 264 & 260 & 16 & 10 \\
\hline East South Central ........ & 173,875 & 185,856 & 1,156 & 1,079 & 3,880 & 3,092 \\
\hline 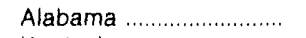 & 52,612 & 48,835 & 128 & 107 & 183 & 236 \\
\hline 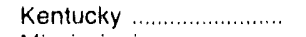 & 66,214 & 73,847 & 112 & 126 & 29 & 40 \\
\hline Mississippi …................ & 8,724 & 12,051 & 738 & 659 & 3,767 & 2.800 \\
\hline 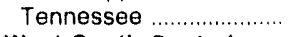 & 46,324 & 51,122 & 178 & 187 & 1 & 16 \\
\hline West South Central ...... & 179,374 & 175,455 & 2,803 & 1,200 & $144,0 i$ & 146,560 \\
\hline 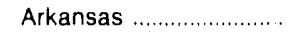 & 18,604 & 19,876 & 144 & 143 & 2,723 & 2,065 \\
\hline Louisiana ......................... & 18,081 & 18,431 & 298 & 272 & 23,522 & 24,286 \\
\hline Oklahoma & 24,122 & 24,273 & 29 & 29 & 17,905 & 17,688 \\
\hline 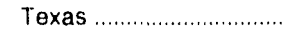 & 118.566 & 112,876 & 2,332 & 756 & 99,861 & 102,521 \\
\hline 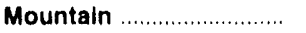 & 184,405 & 180,748 & 781 & 896 & 10,249 & 5,927 \\
\hline Arizona ............................. & 32,364 & 28,391 & 194 & 119 & 4,840 & 2,341 \\
\hline Colorado .......................... & 29,406 & 27,801 & 30 & 39 & 628 & 649 \\
\hline 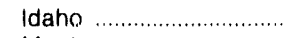 & NA & NA & 2 & $"$ & 0 & 0 \\
\hline Montana ........................... & 16,129 & 16,462 & 30 & 30 & 43 & 37 \\
\hline Nevada ........................... & 15,382 & 16,764 & 377 & 542 & 2,063 & 899 \\
\hline New Mexico .................... & 25,446 & 24,245 & 41 & 45 & 2,630 & 1,979 \\
\hline 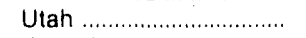 & 29,676 & 28,806 & 48 & 59 & 37 & 5 \\
\hline Wyoming ........................... & 36,003 & 38,279 & 59 & 62 & 9 & 18 \\
\hline Paclfic Contiguous ........ & 8,959 & 8,641 & 9,447 & 7,622 & 53,810 & 54,045 \\
\hline 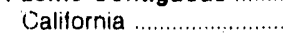 & NA & NA & 9,327 & 7,621 & 51,734 & 53,893 \\
\hline Oregon & 440 & -30 & 35 & -7 & 1,347 & 0 \\
\hline 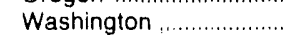 & 8,519 & 8,670 & 85 & 8 & 729 & 153 \\
\hline Pacific Noncontiguous. & 302 & 316 & 8,283 & 7,953 & 2,832 & 2,588 \\
\hline 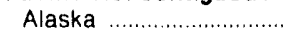 & 302 & 316 & 381 & 356 & 2,832 & 2,588 \\
\hline Hawaii ............................... & NA & NA & 7,902 & 7,597 & 0 & 0 \\
\hline 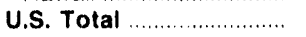 & $1,553,661$ & $1,540,653$ & 158,318 & 148,900 & 266,598 & 252,801 \\
\hline
\end{tabular}

- Value less than 0.5 million kilowatthours.

NA Not applicable.

Notes: -Negative generation denotes that electric power consumed for plant use exceeds gross generation. $\bullet$ Totals may not equal sum of components because of independent rounding.

Source: Energy Information Administration, Form ElA-759, "Monthly Power Plant Report." 
Table 14. Net Generation by Energy Source, Census Dlvision, and State, 1989 and 1988 (Continued)

(Million Kilowatthours)

\begin{tabular}{|c|c|c|c|c|c|c|}
\hline \multirow{2}{*}{$\begin{array}{l}\text { Census Division } \\
\text { State }\end{array}$} & \multicolumn{2}{|c|}{ Nuclear } & \multicolumn{2}{|c|}{ Hydroelectric' } & \multicolumn{2}{|c|}{ Othur $r^{2}$} \\
\hline & 1989 & 1988 & 1989 & 1988 & 1989 & 1988 \\
\hline New Englund ...................... & 33,126 & 32,499 & 4,334 & 3,819 & 502 & 342 \\
\hline Connecticut ...................... & 19,563 & 22,251 & 390 & 324 & 317 & 242 \\
\hline 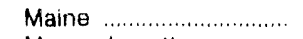 & 6.942 & 5,017 & 1.681 & 1,569 & NA & NA \\
\hline Massachusetts ................. & 3.015 & 1,117 & 226 & 150 & NA & NA \\
\hline New Hampshire ............ & NA & NA & 1,089 & 968 & NA & NA \\
\hline Rhode Island .................... & NA & NA & 0 & 0 & NA & NA \\
\hline Vermont , .......................... & 3,607 & 4.114 & 948 & 809 & 184 & 100 \\
\hline Middle Allantic & 85,045 & 85,828 & 24,947 & 24,384 & NA & 0 \\
\hline New Jersey ...................... & 23,032 & $2: 3,890$ & -261 & -222 & NA & NA \\
\hline Now York ....................... & 22,847 & 24,175 & 23,918 & 23,901 & NA & 0 \\
\hline Pennsylvania ..................... & 39,166 & 37,862 & 1,290 & 705 & NA. & NA \\
\hline East North Central ........ & 119,641 & 106,893 & 2,698 & 2,389 & 498 & 514 \\
\hline Illinois .............................. & 74,820 & 69,166 & 50 & 48 & NA & NA \\
\hline Indiana ............................... & NA & NA & 450 & 441 & NA & NA \\
\hline Michigan .............................. & 21,312 & $17 ; 808$ & 749 & 483 & 0 & 0 \\
\hline 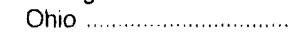 & 12,661 & 8,455 & 130 & 187 & 316 & 351 \\
\hline 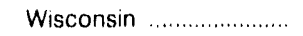 & 10,848 & 11,464 & 1,319 & 1,230 & 181 & 164 \\
\hline West North Central ....... & 40,196 & 37,865 & 10,064 & 11,240 & 271 & 210 \\
\hline 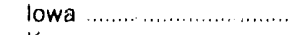 & 3,139 & 3,163 & 672. & 698 & 24 & 57 \\
\hline Kansas ............................. & 9,709 & 6,650 & 10 & 12 & $\cdot$ & • \\
\hline Minnesola ....................... & 10,926 & 12,288 & 654 & 532 & 247 & 153 \\
\hline 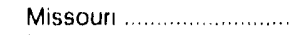 & 8,344 & 8,935 & 1,094 & 1,511 & NA & NA \\
\hline Nebraska .............................. & 8,077 & 6,828 & 1,158 & 1,350 & NA & NA \\
\hline North Dakola ....................... & NA & NA & 1,893 & 1,884 & NA & 0 \\
\hline South Dakota & NA & NA & 4,583 & 5,254 & NA & NA \\
\hline South Atlantic & 132,852 & 144,010 & 15,775 & 7,149 & 14 & 57 \\
\hline Delaware ............................ & NA & NA & NA & NA & NA & NA \\
\hline District of Columbia ...... & NA & NA & NA & NA & NA & NA \\
\hline Florida ...................... & 20,916 & 26,198 & 234 & 209 & NA & 0 \\
\hline Georgia & 24,961 & 15,149 & 3,874 & 2,011 & NA & NA \\
\hline Maryland .......................... & 2.719 & 11,734 & 1.778 & 1,328 & 14 & 57 \\
\hline North Carolina .................... & 29,212 & 29,146 & 6,996 & 2,890 & NA & NA \\
\hline South Carolina & 40,780 & 40,746 & 2,016 & $63 i$ & NA & NA \\
\hline Virginia ............................. & 14,264 & 21,037 & 401 & -218 & $\cdot$ & $\cdot$ \\
\hline West Virginia & NA & NA & 476 & 297 & NA & NA \\
\hline East South Central ........ & 34,954 & 26,504 & 29,410 & 12,374 & NA & NA \\
\hline Alabama & 11,524 & 12,981 & 13,153 & 5,359 & NA & NA \\
\hline Kentucky .......................... & NA & NA & 4,404 & 2,423 & NA & NA \\
\hline Mississippi ........................ & 7,826 & 9,582 & NA & NA & NA & NA \\
\hline Tennessee ......................... & 15,603 & 3,940 & 11,853 & 4,591 & NA & NA \\
\hline West South Central & 31,225 & 26,473 & 6,917 & 6,065 & 190 & 0 \\
\hline Arkansas .......................... & 8,844 & 8,885 & 3,084 & 2.785 & NA & NA \\
\hline I. ouisiana ....................... & 12,391 & 13,785 & NA & NA & NA & NA. \\
\hline Oklahoma & NA & NA & 2,392 & 2,045 & NA & NA \\
\hline Texas & 9,990 & 3,792 & 1,441 & 1,235 & 190 & 0 \\
\hline Mountain & 8,380 & 23,600 & 31,392 & 28,072 & 246 & 232 \\
\hline ………........ & 7,850 & 22,940 & 7,877 & 7,771 & NA & NA \\
\hline Colorado & 529 & 660 & 1,705 & 1.744 & 1 & 2 \\
\hline Idaho & NA & NA & 8,927 & 6,745 & NA & NA \\
\hline 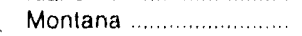 & NA & NA & 9,550 & 8,237 & 72 & 55 \\
\hline Nevada & NA & NA & 1,859 & 2,091 & NA & NA \\
\hline New Mexico ....................... & NA & NA & 232 & 100 & NA & NA \\
\hline 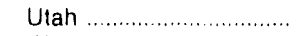 & NA & NA & 562 & 593 & 173 & 174 \\
\hline Wyoming & NA & NA & 680 & 789 & - & * \\
\hline Pacitic Contiguous ......... & 43,936 & 43,202 & 138,633 & 126,499 & 9,565 & 10,613 \\
\hline California & 32,519 & 30,863 & 29,524 & 23,474 & 9,161 & 10,131 \\
\hline Oregon ….......................... & 5,299 & 6,339 & 37,815 & 34,646 & 28 & 99 \\
\hline Washinglon ......................... & 6,118 & 6,000 & 71,293 & 68,379 & 376 & 383 \\
\hline Pacific Noncuritiguous & NA & NA & 895 & 950 & 25 & 16 \\
\hline Alaska .............................. & NA & NA & 873 & 935 & 0 & 0 \\
\hline 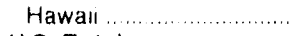 & NA & NA & 22 & 14 & 25 & 16 \\
\hline U.S. Totai & 529,355 & 526,973 & 265,063 & 222,940 & 11,309 & 11,984 \\
\hline
\end{tabular}

- Station losses include energy used for pumped storage. Energy used in 1989 for pumping was 24,964 millon kilowatthours and in 1988 was 23,935 million kilowatthours.

2 Includes geothermal, wood, wind, waste, and solar.

- Value less than 0.5 million kilowatthours.

NA. Not applicable.

Notes: - Negative generation denotes that electric power consumed for plant use exceeds gross generation. -Totals may not equal sum of components because of independent rounding.

Source: Energy Information Administration, Form EIA-759, "Monthly Power Plant Report." 
Table 15. Petroleum-Fired Net Generation by Selected Prime Mover, Census Division, and State, 1989 and 1988

(Million Kilowatthours)

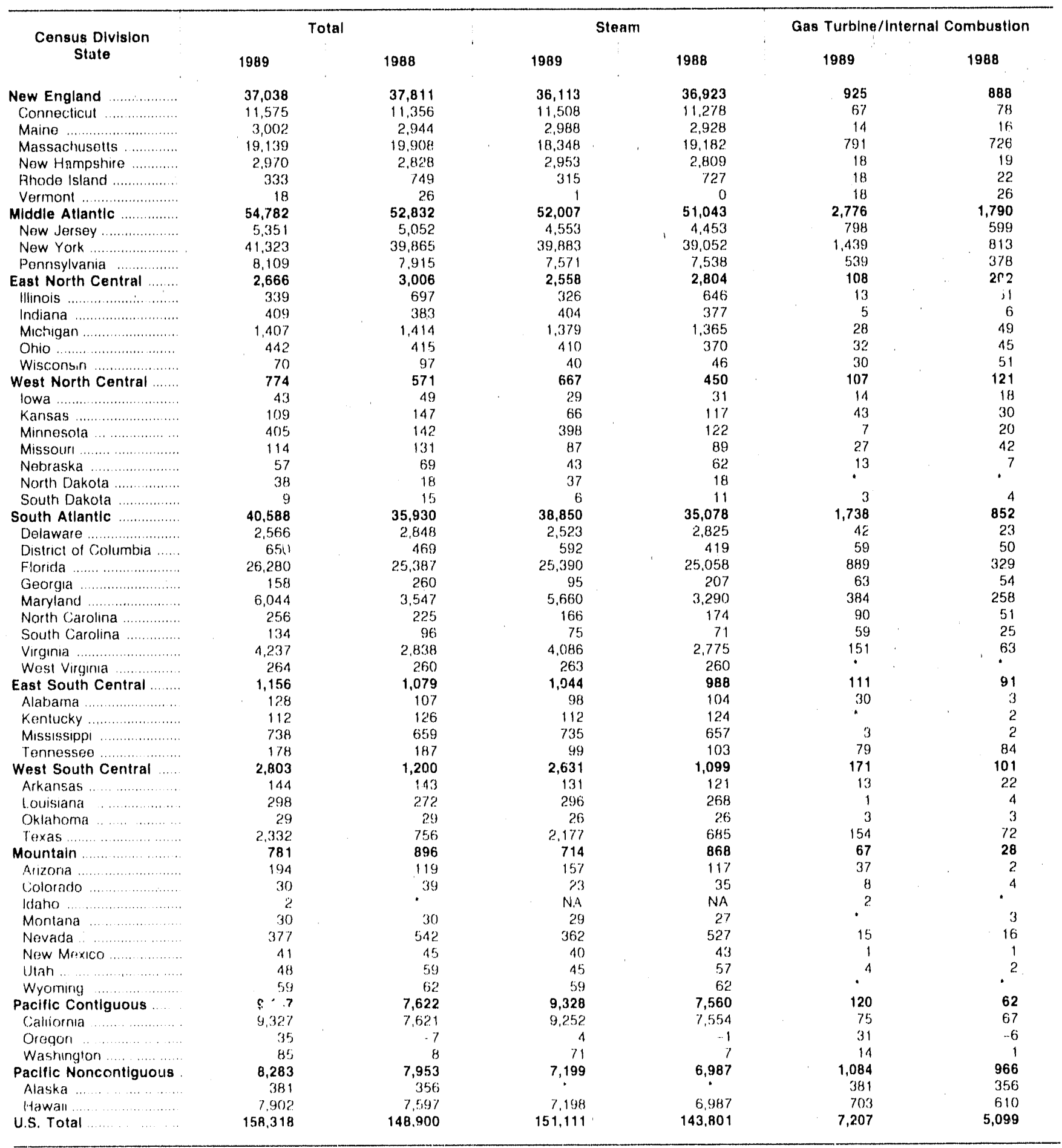

- Value less than 0.5 million kilowatthours

NA Nol applicable

Notes: - Nequative generation denotes that electric power consumed for plant use exceeds gross generation. - Tolals may liat equal sum of comporienls tecause of inderendent rounding.

Source Enorgy Information Adrninistration, Form EIA.759. "Monthly Power Planil Roport." 
Table 16. Gas-rirred Net Generation by Selected Prime Mover, Census Division, and State, 1989 and 1988

(Million Kilowatthours)

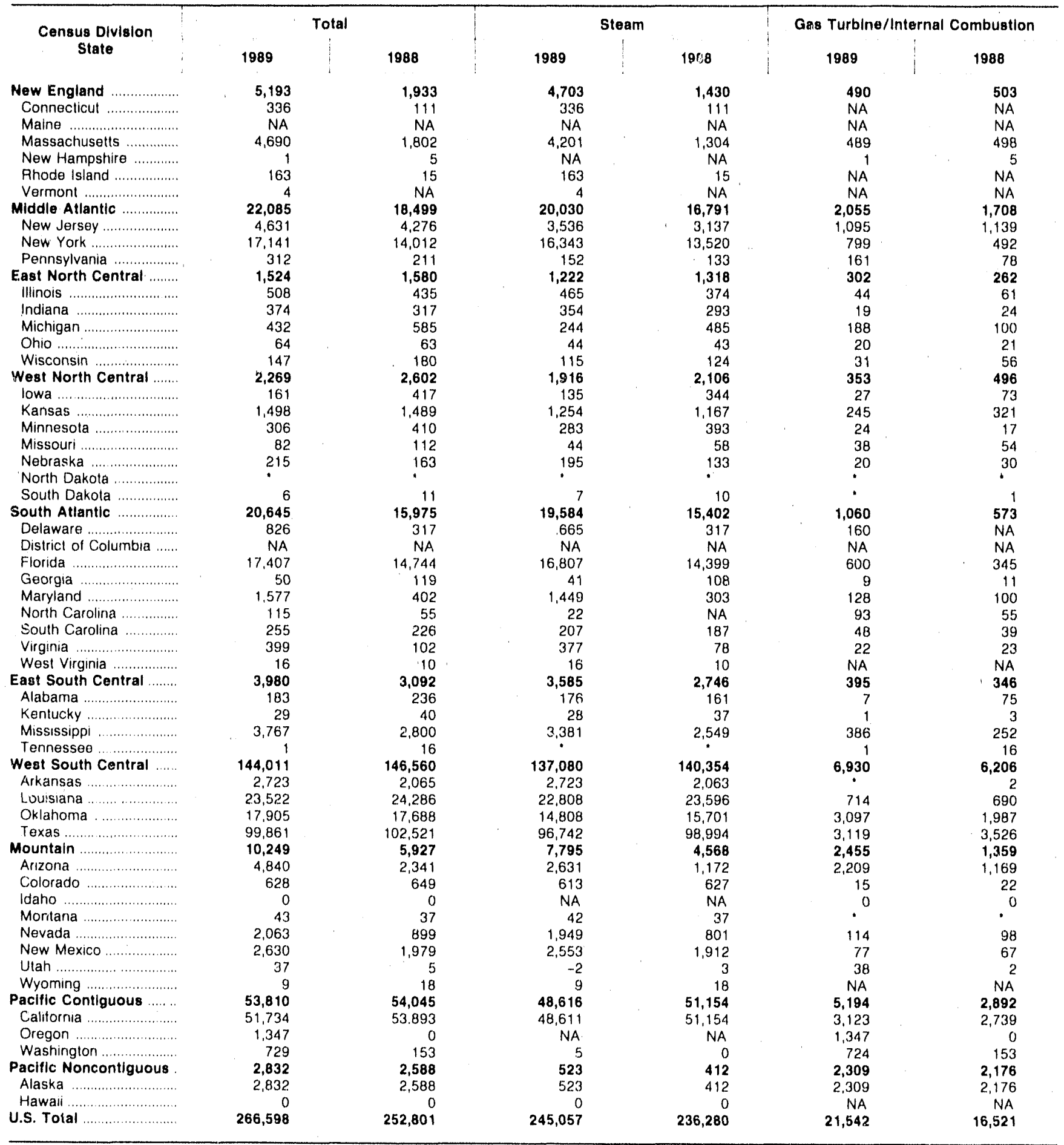

- Value less than 0.5 million kilowatthours

NA : Not applicable.

Notes: - Negative gengration denotes that electric power consumed for plant use exceeds gross generation. - $r o t a l s$ may not equal surn of components because of independent rounding.

Source: Energy Information Administration, Form ElA-759, "Monthly Power Plant Report." 


\section{Fossil-Fuel Statistics}

This chapter contains statistics on the consumption of fossil fuels used to generate alectricity, end-of-ycar fossil-fuel stocks for all electric utility plants, and the receipts and costs of fossil fuels at stcam-electric power plants with a nameplate capacity of 50 or more megatwatts. These data are aggregated to the national, Census division, and State levels.

\section{Background}

Various sources of energy are used by the electric power industry to produce electricity; however, fossil fuels supply about 70 percent of the energy sources for the generation requirements of the Nation. Fossil fuels are so named because they are derived from the remains of the plants and animals, which lived on earth millions of years ago. Coal, petroleum, and gats are currently the dominant fossil fucls used by the industry Other fossil fuels include petroleum coke, refincry gas, coke oven gas, blast furnace gas, and liquified petroleum gas. Statistics on consumption, purchases (receipts), and stocks of fossil fuels at electric utilities are interdependent. That is, the stocks on site at the utility at the end of the current year result from the stocks that were availat,le at the end of the prior year, the amount of fuel purchased during the current year, and the amount of fuel consumed during the year.

\section{Fossil-Fuel Consumption and Stocks}

Coal, the energy source used to gencrate more than one-half of the electricity needed in the Nation, is consumed extensively throughout the United States (particularly in the East North Central, West North Central, West South Central, and the South Atlantic Census Divisions). The use of petroleum for generation is not as common on a national level as during the early 1970's; however, some areas of the country (such as in California, as well as in the New England and Pacific Noncontiguous Census Divisions) continue to use it extensively. Consumption of gas occurs mosily in areas of the country where it is readily accessible, particularly in the West South Central Census Division, and in dual-fired generator units, which use gas and petroleum as substitute fucls.

The purposes of on-site storage are 10 provide an uninterrupted supply, allow bulk shipments, and take advantage of favorable market conditions. Since gas is generally not stored, there are no stoctis of gas. Electric utilities maintain stockpiles of coal and petroleum to minimize the effect of an interruption or curtailment in fuel availability for example, railroad strikes, coalmine strikes, or oil embargoes).

\section{Fossil-Fuel Receipts and Costs}

Statistics on receipts provide information regarding the delivery of fossil fuels o steam-electric plants. The costs include all costs incurred by an electric utility in the purchase and delivery of fuel to the plant. The type of contract under which a fuel is purchased has a significint effect on the cost of the fuel delivered and can be used as a good indicator of market conditions. Transactions where petroleum and eoal are obtained by the utility under purchase orders or contracts of more than 1-year duration are referred to as contract purchases. Shipments of petroleum and coal under purchase orders or contracts of less than 1-year duration are considered spot purchases. Transactions to secure gas that are conducted under a contract with uninterrupted do. livery are identified as firm purchases. Interruptible "run. chases are those in which the gas is received under is contract that permits curtailment of service under certain circumstances. For example, under both Federal and State regulations, requirements for gas to heat homes and serve industry have priority over requirements of the electric power industry. Consequently, a contract under which gas is purchased is most generally one that allows for an interuption in its accessibility. A contract under which gas is purchased and delivered only at a specified time of the year is an off-peak purchase.

Coal. Coal is obtained from three major coalproducing areas in the United States. Appalachion coal is mined in both surface and underground mines located in Pemsylvania, Maryland, Virginia, West Virginia, eastern Kentucky, Temnessee, Alabama, and Ohio. This coal is bituminous in rank and of low-to-medium sulfur content. Its heat content in British Thermal Units $(B(1)$ averages over $12,0(0)$ Btu per pound. The coal is transported primarily by train, barge, and truck to electric plants throughout the castern United States. Interior coal is mined in both surface and underground mines liseated in Illinois, Indiana, western Kentucky, and Missouri. It is bituminous coal with a high percentage of 'sulfur and ash and contains approximalely $11,()(0)$ Btu per pound. Most of this conal is delivered to electric 
plants in the central United States. Western coal is mined in Montana, Wyoming, Colorado, Utah, North Dakota, Arizona, and New Mexico. It is delivered to electric plants throughout the western and central United States. Over one-half of the coal in this region is subbituminous coul that is low in sulfur content (less than 0.5 percent per pound) and contains approximately 8,000 Btu per pound. This coal originates in the Powder River basin of northeast Wyoming and southeast Montana. Coal from this region is delivered by unit train to electric plants as far east as Ohio and Georgia.

The cost of coal delivered to electric utilities can vary significantly from State to State. Coal delivered to the New England Census Division from the Appalachian coal fields may cost as much as $\$ 60.00$ per short ton due to transportation costs and the higher cost of producing eastern coal, which is generally in underground mines. Environmental restrictions within a State may require electric utilities to burn only the more expensive, low-sulfur coal resulting in a higher delivered cost. In the West, especially in the Mountain Census Division, coal-burning plants are often built in close proximity to the mine thus reducing transportation costs. In addition, the cost of mining coal from the large surface mines located in the western United States is significantly less than that of underground eastern mines, resulting in a delivered cost of under $\$ 15.00$ per short ton for States such as Montana and Wyoming. In States such as North Dakota, South Dakola, and Texas, the cost of coal delivered to electric utilities is well below the national average because of the lower cost of low-grade lignite.

Petroleum. Although nationwide receipts at electric utilities are less than one-half the volume of the 1970's, several electric utilities in the New England area, and in New York, Florida, California, and Hawaii still depend on petroleum for a significant portion of their fossil-fuel requirements. Receipts can vary widely from year to year at electric utilities due to changes in the cost of petroleum. Fuel oil Numbers 2, 4, 5, and 6 are purchased by electric utilities for use in generating electricity. Fuel oil Numbers 4, 5, and 6 (heavy oil) constitute the majority of all petroleum receipts at electric utilities. Smaller amounts of fuel oil Number 2 (light oil) are also used by electric utilities primarily for start-up and flame stabilization of the boilers.

The cost of petroleum delivered to electric utilities varies considerably from State to State. The most important factor in determining cost is the type of fuel oil that is being delivered. States receiving only lowgrade heavy oil will show a delivered cost much lower than a State receiving only light oil. Most of the petroleum delivered to the New England, Middle Atlantic, and South Atlantic C'ensus Divisions, California, and Hawaii for use by electric utilities is the Number 6 fuel oil. The cost of fued oil can vary hecause of its sulfur content. Belectric utilities that are required to meet stringent envirommental standareds hust purchase lowsulfur fucl oil al premium prices.

Gas. Gas is used extensively as a primary luel throughout areas of the country where it is readily aceessible. for example, the West South Central Census Division and in California. Large volumes of gats are also transported by pipeline to the Middle Allantic, and South Allantic Census Divisions. Gas receipts to these Census divisions and to Califormia can vary considerably from year to year because some electric utilities switch between use of petroleum and gas in clual-fired generating units. The highest volume of gas receipts at electric utilities occurs during the summer months when demand for electricity peaks and when there is a greater amount available to electric utilities because of lower denands from residential and commercial consumers. In some northern parts of the United States, receipts of gas at electric utilities are limited during the winter months due to the priority for heating and industry needs. Many electric utilities have the capability of burning either petroleum or gas. The cost of the fuel is usually the determining factor. One major advantage of gas over all other fossil fuels is that it is a clean burning fuel. Therefore, some electric utilities use gas in order to comply with envirommental regulations.

Petroleum Coke. Petroleum coke is the final product or residue produced from the process of breaking down complex petroleum hydrocatbons into lighter petroleum products such as gasoline (cracking).

\section{Data Sources}

Data in the following tables were obtained from two sources. The first is the Form EIA-759, "Monthly Power Plant Report," which is used to collect monthly data from all operators of electric utilities (approximately $8(0))$ in the United States. Moredetailed statistics on stocks and consumption are published in the Electric Power Monthly." The second source is the Federal Energy Regulatory Commission (FERC) Form 423, "Monthly Report of Cost and Quality of Fuels for Electric Plants," which is a restricted-universe census used to collect data from approximately 225 electric utilities. More detailed statistics on receipts and costs of fossil fuels are published in the Cost and Quality of Fusels for Electric Utility Plants." Caution should be used in comparing stocks, receipts, and consumption data since all operators of power plants are surveyed by the Form EIA-759, while the FERC Form 423 is limited to operators of power plants with a fossil-fueled steam-electric nameplate capacity of $5($ ) or more megawatts.

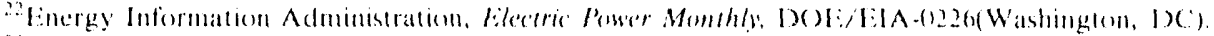

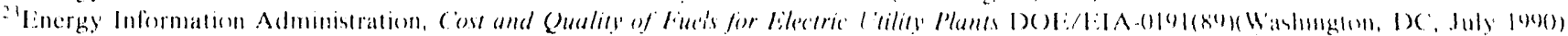


Table 17. Consumption of Fossil Fuels, and Stocks of Coal and Petroleum, 1985-1989

\begin{tabular}{|c|c|c|c|c|c|}
\hline Item & 1989 & 1988 & 1987 & 1986 & 1986 \\
\hline \multicolumn{6}{|l|}{ Consumptlon } \\
\hline Coal (thousand short tons) ...... & 766,888 & 758,372 & 717,894 & 685,056 & 693,841 \\
\hline 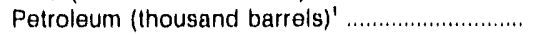 & 267,451 & 248,096 & 199,378 & 230,482 & 173,414 \\
\hline 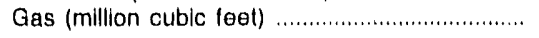 & $2,787,012$ & $2,635,613$ & $2,844,051$ & $2,602,370$ & $3,044,083$ \\
\hline \multicolumn{6}{|l|}{ Stocks ${ }^{2}$} \\
\hline Coal (thousand short tons) ........ & 135,860 & 146,507 & 170,797 & 161,806 & 156,376 \\
\hline 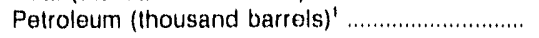 & 61,270 & 69,285 & 70,827 & 73,111 & 73,689 \\
\hline
\end{tabular}

1 Does not include petroleum coke.

2 Stocks are shown as of December 31 of each year.

Note: Totals may not equal sum of components because of independent rounding.

Source: Energy Information Administration, Form EIA-759, "Monthly Power Plant Report."

Table 18. Receipts and Average Delivered Cost of Fossil Fuels, 1985-1989

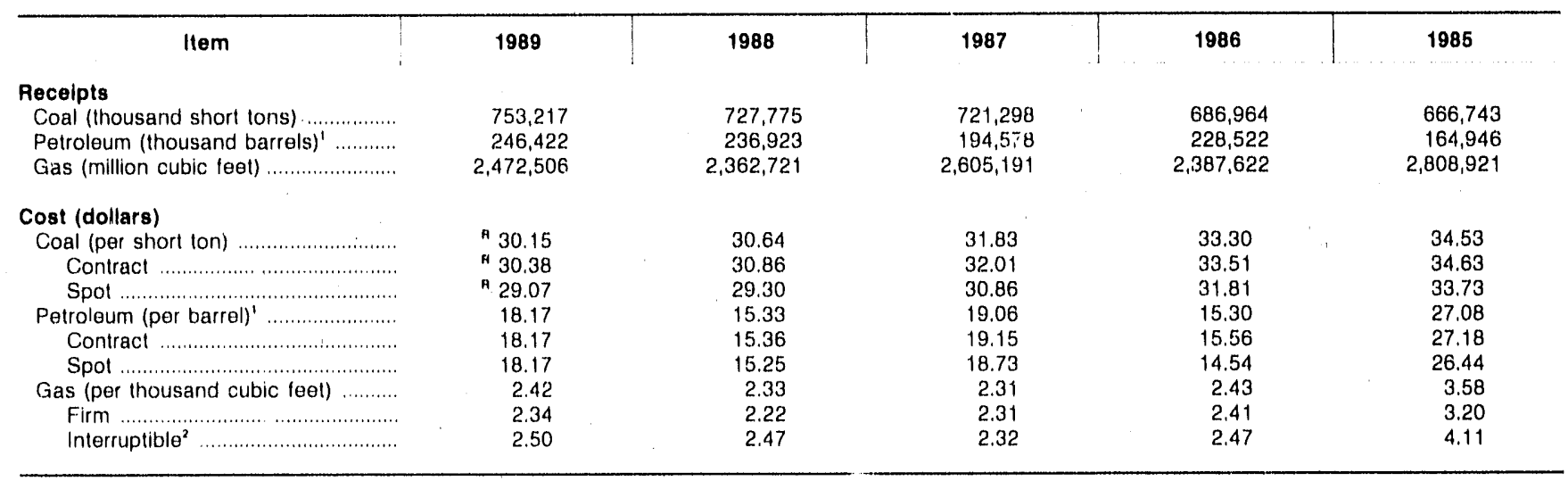

Does not include petroleum coke

2 Includes off-peak gas.

A ... Revised data.

Notes: Data are for plants with a fossil-fueled steam-electric nameplate capacity of 50 or more megawatts. -Totals may not equal sum of components because of independent rounding.

Source: Federal Energy Regulatory Commission, FERC Form 423, "Monthly Report of Cost and Quallty of Fuels for Electric Plants."

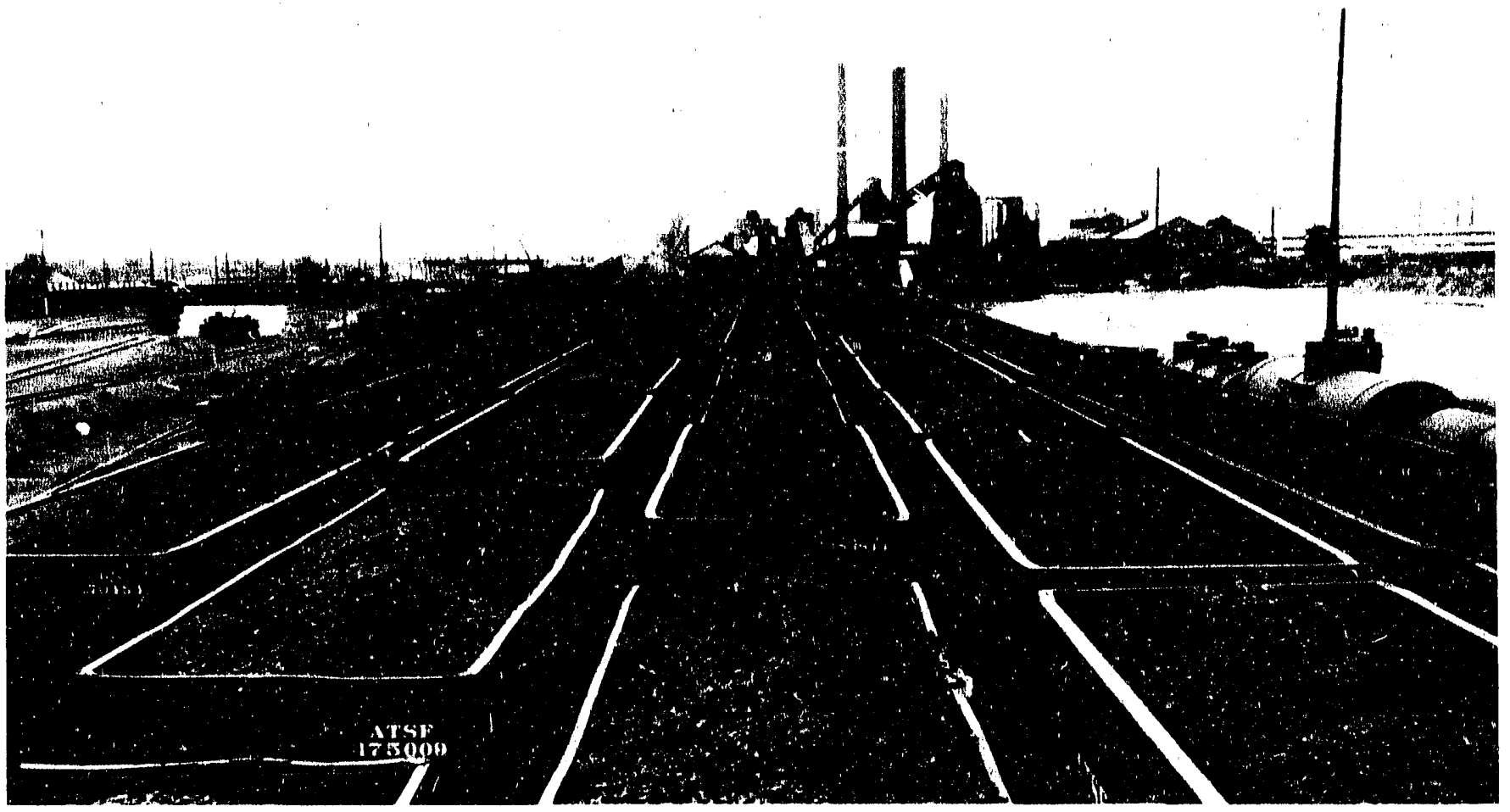

The average cost of coal includes the cost of transporting the coal from the mine to the utility. 
Table 19. Consumption of Fossil Fuels by Census Divișion and State, 1989 and 1988

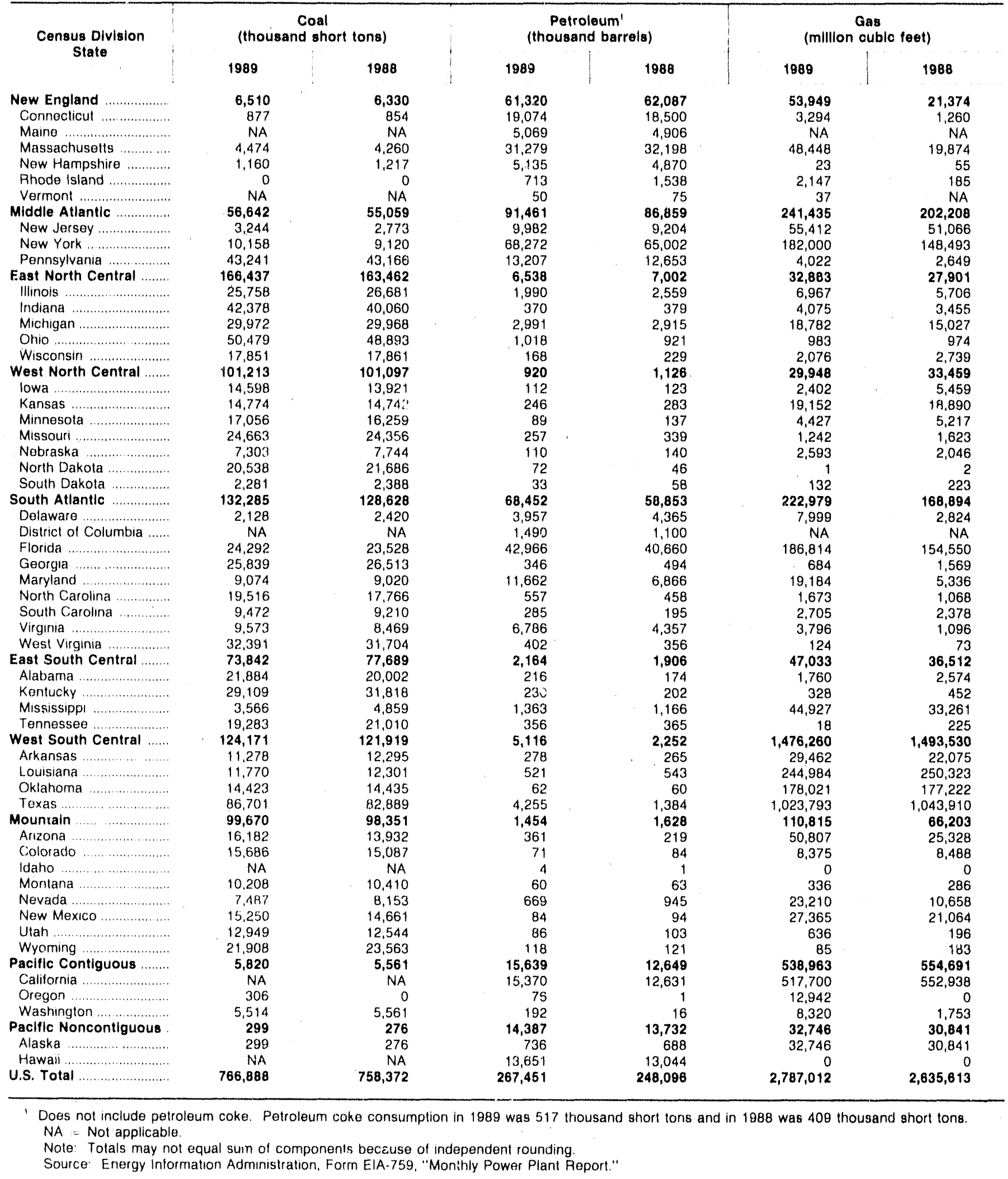


Table 20. Petroleum Consumption by Selected Prime Mover, Census Division, and State, 1989 and 1988

(Thousand Barrels)

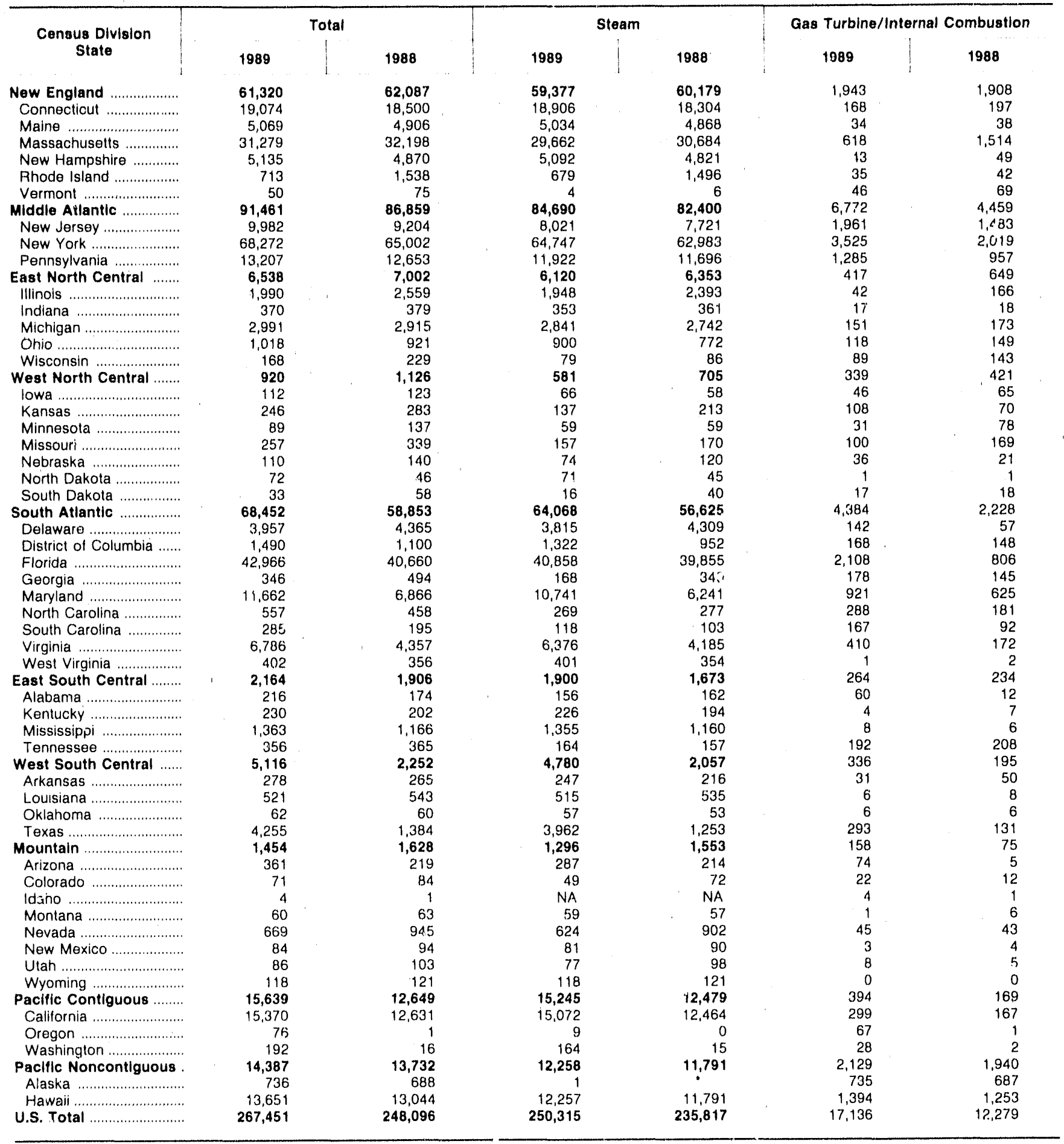

- Value less than 0.5 .

NA : Not applicable.

Notes: - Totals may not equal sum of components because of independent rounding. - Does not include petroleum coke. Petroleum coke consumption in 1989 was 517 thousand short ions and in 1988 was 409 thousand short tons.

Source: Energy Information Administration, Form EIA.759, "Monthly Power Plant Report." 
Table 21. Gas Consumption by Selected Prime Mover, Census Division, and State, 1989 and 1988

(Million Cubic Feet)

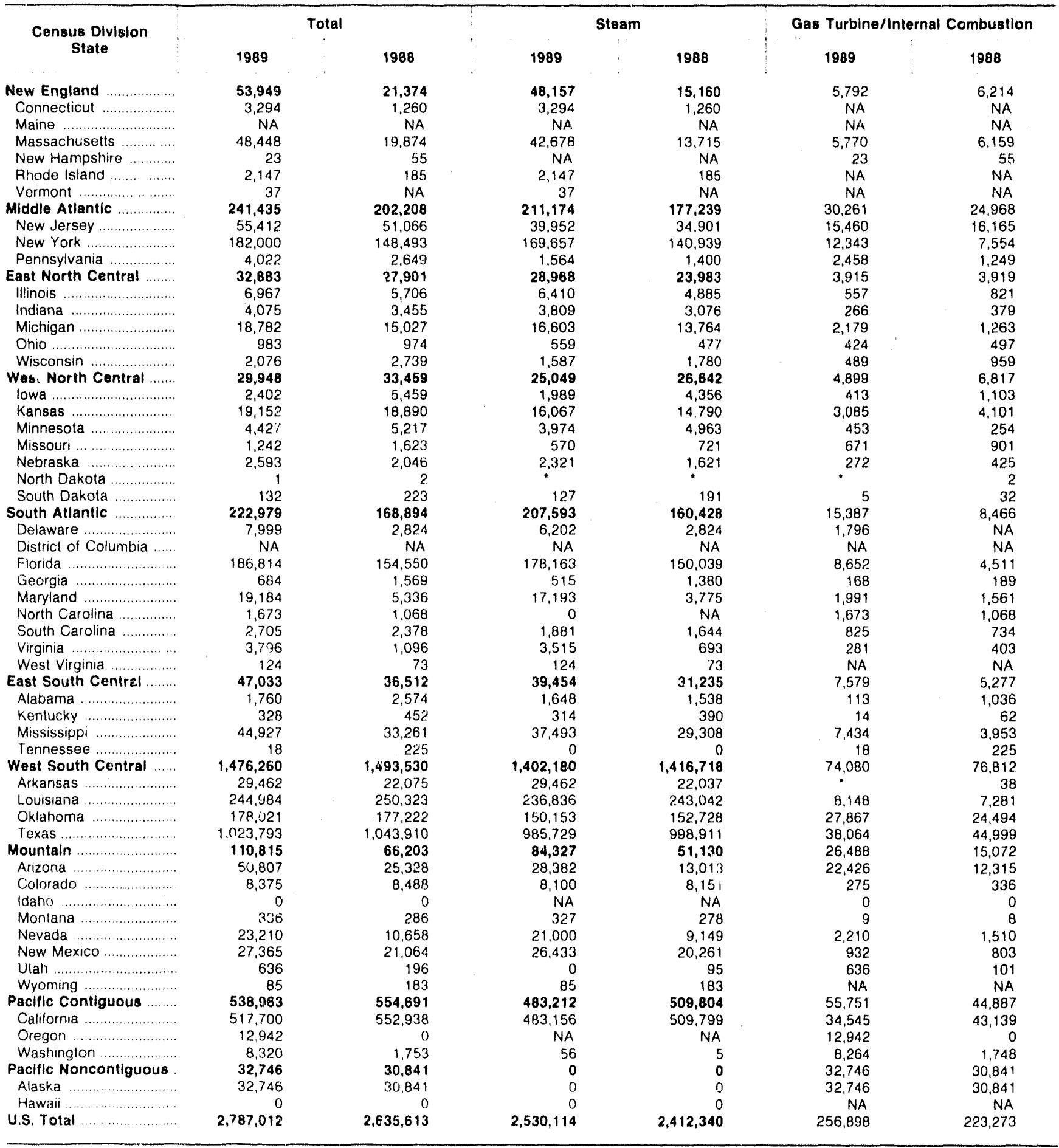

- Value less than 0.5

NA Not applicable.

Note: Totals may not equal sum of components because of independent rounding.

Source: Energy information Administration, Form ElA-759, "Monthly Power Plant Report." 
Table 22. Coal and Petroleum Stocks by Census Division and State, as of December 31, 1989 and 1988

\begin{tabular}{|c|c|c|c|c|}
\hline \multirow{2}{*}{$\begin{array}{l}\text { Census Division } \\
\text { State }\end{array}$} & \multicolumn{2}{|c|}{$\begin{array}{c}\text { Coal } \\
\text { (thousand short tons) }\end{array}$} & \multicolumn{2}{|c|}{$\begin{array}{c}\text { Petroleum' } \\
\text { (thousand barrels) }\end{array}$} \\
\hline & 1989 & 1988 & 1989 & 1988 \\
\hline New England & 1,058 & 1,110 & 5,521 & 5,424 \\
\hline $\begin{array}{l}\text { Connecticut } \\
\text { Maine }\end{array}$ & 145 & 0 & 2,241 & 2,122 \\
\hline $\begin{array}{l}\text { Maine } \\
\text { Massachusetts }\end{array}$ & NA & NA & 618 & 647 \\
\hline $\begin{array}{l}\text { Massachusetts } \\
\text { New Hampshire }\end{array}$ & 642 & 737 & 2,228 & 2,167 \\
\hline $\begin{array}{l}\text { New Hampshire } \\
\text { Rhode Island }\end{array}$ & 243 & 344 & 323 & 313 \\
\hline $\begin{array}{l}\text { Phode Island } \\
\text { Vermont }\end{array}$ & 28 & 28 & 74 & 135 \\
\hline $\begin{array}{l}\text { Vermont } \\
\text { Middle Atlantic }\end{array}$ & NA & NA & 38 & . 41 \\
\hline $\begin{array}{l}\text { Middle Atlantic } \\
\text { New Jersey }\end{array}$ & 12,934 & 13,757 & 13,297 & 14,980 \\
\hline $\begin{array}{l}\text { New Jersey } \\
\text { New York }\end{array}$ & 632 & 528 & 2,510 & 2,848 \\
\hline $\begin{array}{l}\text { New York } \\
\text { Pennsylvania }\end{array}$ & 1,233 & 1,432 & 9,143 & 10,336 \\
\hline 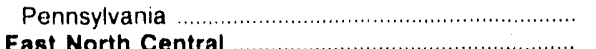 & 11,069 & 11,798 & 1,644 & 1,796 \\
\hline 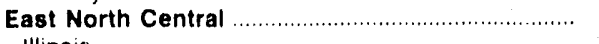 & 34,948 & 39,549 & 3,218 & 3,424 \\
\hline $\begin{array}{l}\text { Illinois . } \\
\text { Indiana }\end{array}$ & 8,204 & 9,179 & 1,951 & 1,790 \\
\hline 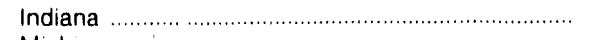 & 8,043 & 9,997 & 178 & 185 \\
\hline $\begin{array}{l}\text { Michigan } \\
\text { Ohio }\end{array}$ & 8,185 & 8,991 & 617 & 876 \\
\hline $\begin{array}{l}\text { Onio } \\
\text { Wisconsin }\end{array}$ & 6,607 & 7,218 & 283 & 377 \\
\hline $\begin{array}{l}\text { Wisconsin } \\
\text { West North Central }\end{array}$ & 3,909 & 4.165 & 190 & 196 \\
\hline 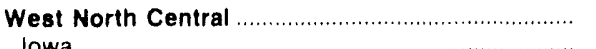 & 19,356 & 19,950 & 1,647 & 1,865 \\
\hline lowa & 4,044 & 4,456 & 198 & 227 \\
\hline 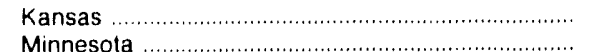 & 3,266 & 3,300 & 601 & 668 \\
\hline $\begin{array}{l}\text { Minnesota } \\
\text { Missouri }\end{array}$ & 2,052 & 2,579 & 130 & 135 \\
\hline $\begin{array}{l}\text { Missouri } \\
\text { Nebraska }\end{array}$ & 4,275 & 4,541 & 362 & 413 \\
\hline $\begin{array}{l}\text { Nebraska } \\
\text { North Dakota }\end{array}$ & 1,685 & 1,593 & 215 & 293 \\
\hline $\begin{array}{l}\text { North Dakota } \\
\text { South Dakota }\end{array}$ & 3,731 & 3,166 & 60 & 57 \\
\hline South Dakota & 303 & 314 & 80 & 73 \\
\hline South Atlantic & 20,493 & 23,741 & 11,547 & 11,788 \\
\hline 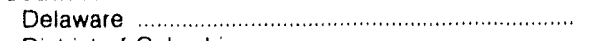 & 259 & 498 & 537 & 505 \\
\hline District of Columbia & NA & NA & 93 & 121 \\
\hline Florida & 4,383 & 4,804 & 6.457 & 6,562 \\
\hline Georgia & 5,040 & 5,288 & 483 & 604 \\
\hline Maryland & 1,046 & 1,476 & 1,290 & 1,374 \\
\hline North Carolina & 2,795 & 3,727 & 206 & 227 \\
\hline 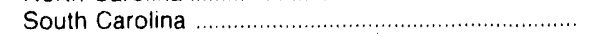 & 1,873 & 1,661 & 470 & 430 \\
\hline Virginia & 1,368 & $1,2 i 1$ & 1,814 & 1,808 \\
\hline West Virginia & 3,729 & 5,077 & 198 & 156 \\
\hline East South Central & 11,651 & 13,351 & 1,174 & 1,580 \\
\hline Alabama & 3,721 & 4,462 & 88 & 156 \\
\hline Kentucky . . & 4,299 & 4,124 & 122 & 126 \\
\hline 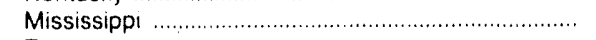 & 754 & 870 & 708 & 861 \\
\hline Tennessee & 2,875 & 3,895 & 256 & 437 \\
\hline West South Central & 16,917 & 16,272 & 6,933 & 8,388 \\
\hline 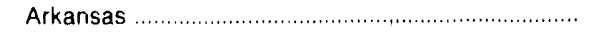 & 2,134 & 1,899 & 251 & 247 \\
\hline 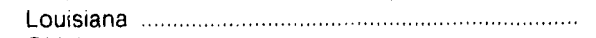 & 2,627 & 2,772 & 1,209 & 1,351 \\
\hline Oklahoma & 2,826 & 2,678 & 440 & 455 \\
\hline Texas & 9,329 & 8,922 & 5,032 & 6,336 \\
\hline Mountain & 17,035 & 17,079 & 1,774 & 1,899 \\
\hline 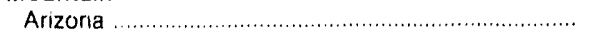 & 3,367 & 4,187 & 789 & 848 \\
\hline Colorado & 3,921 & 3,763 & 255 & 28.3 \\
\hline Idaho & NA & NA & 14 & 8 \\
\hline 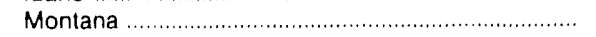 & 813 & 913 & 13 & 20 \\
\hline Nevada & 993 & 1,434 & 482 & 460 \\
\hline New Mexico ……………………………………… & 1,403 & 1,392 & 155 & 210 \\
\hline Utah & 3.202 & 2,772 & 35 & 34 \\
\hline 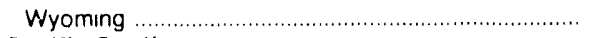 & 3,337 & 2,619 & 31 & 36 \\
\hline Pacific Contiguous . & 1,466 & 1,696 & 14,814 & 18,795 \\
\hline California & NA & NA & 14,470 & 18,401 \\
\hline Oregon & 480 & 786 & 228 & 149 \\
\hline 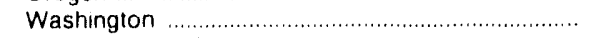 & 986 & 910 & 116 & 246 \\
\hline Pacific Noncontiguous & 3 & 3 & 1,344 & 1,141 \\
\hline Alaska & 3 & 3 & 236 & 243 \\
\hline 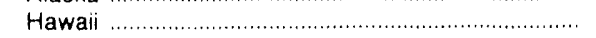 & NA & NA & 1,109 & 899 \\
\hline U.S. Total & 135,860 & 146,507 & 61,270 & 69,285 \\
\hline
\end{tabular}

1 Does not include petroleum coke. Petroleum coke stocks at the end of 1989 were 105 thousand short tons and in 1988 were 86 thousand short tons.

NA Not applicable

Note: Totals may not equal sum of components because of independent roundirig.

Source: Energy Intormation Administration, Form ElA-759, "Monthly Power Plant Report." 
Table 23. Fossil-Fuel Receipts by Census Division and State, 1989 and 1988

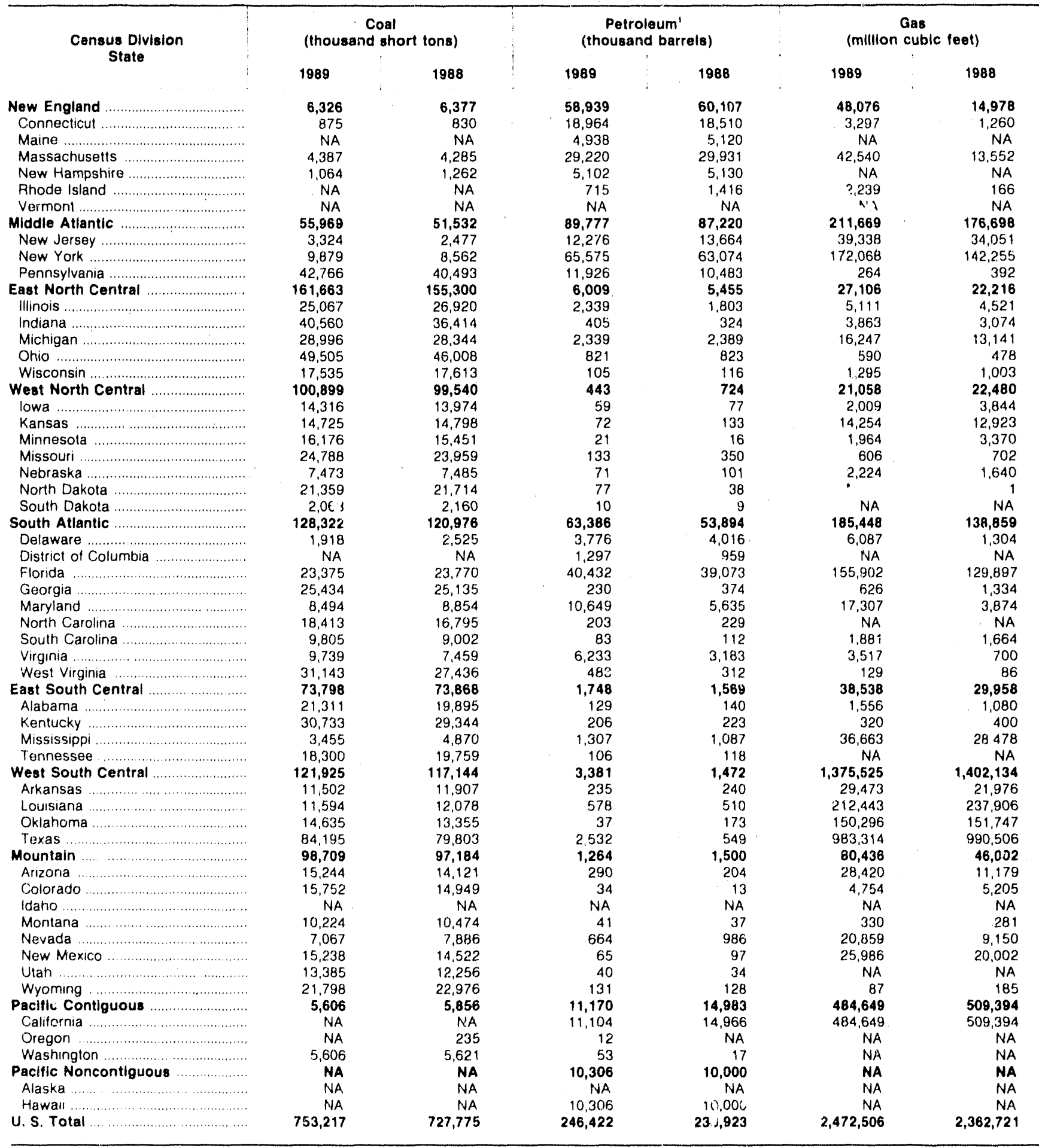

- Does not include petroleum coke. Petroleum coke receipts in 1989 were 495 thousand short tons and in 1988 were 356 thousand short tons

- Value less than 0.5 .

NA ... Not applicable.

Notes: Data are for plants with a fossil-fueled sleam-electric nameplate capacity of 50 or more megawalts. -Totals may not equal sum of components because of independent rounding.

Source: Federal Energy Regulatory Commission, FERC Form 42's. "Monthly Report of Cost and Quality of Fuels for Electric Plants." 
Table 24. Average Delivered Cost of Fossil-Fuel Receipts by Census Division and State, 1989 and 1988

\begin{tabular}{|c|c|c|c|c|c|c|c|c|c|}
\hline \multirow{3}{*}{$\begin{array}{c}\text { Census Division } \\
\text { State }\end{array}$} & \multicolumn{3}{|c|}{ Coal } & \multicolumn{3}{|c|}{ Petroleum' } & \multicolumn{3}{|c|}{ Gas } \\
\hline & \multicolumn{2}{|c|}{1989} & \multirow{2}{*}{$\begin{array}{c}1988 \\
\text { (cents } \\
\text { per } \\
10^{\circ} \text { Btu) }\end{array}$} & \multicolumn{2}{|c|}{1989} & \multirow{2}{*}{$\begin{array}{c}1988 \\
\text { (cents } \\
\text { per } \\
10^{6} \text { Btu) }\end{array}$} & \multicolumn{2}{|c|}{1989} & \multirow{2}{*}{$\begin{array}{c}1988 \\
\text { (cents } \\
\text { per } \\
10^{6} \text { Btu) }\end{array}$} \\
\hline & $\begin{array}{l}\text { (s per } \\
\text { short } \\
\text { ton) }\end{array}$ & $\begin{array}{c}\text { (cents } \\
\text { per } \\
10^{\circ} \text { Btu) }\end{array}$ & & $\begin{array}{l}\text { (\$ per } \\
\text { barrel) }\end{array}$ & $\begin{array}{c}\text { (cents } \\
\text { per } \\
10^{\circ} \text { Btu) }\end{array}$ & & $\begin{array}{l}\text { (\$ per } \\
\text { Mcf) }\end{array}$ & $\begin{array}{c}\text { (cents } \\
\text { per } \\
10^{6} \text { Btu) }\end{array}$ & \\
\hline New England & 46.54 & 169.9 & 173.2 & 17.04 & 269.2 & 227.5 & 2.50 & 238.5 & 221.6 \\
\hline 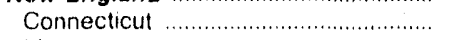 & 56.88 & 213.7 & 228.6 & 18.01 & 285.6 & 239.7 & 2.59 & 251.1 & 217.4 \\
\hline 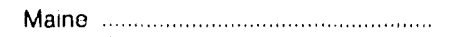 & NA & NA & NA & 17.48 & 278.2 & 235.3 & NA & NA & NA \\
\hline 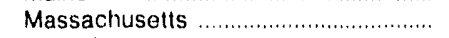 & 41.68 & 160.2 & 161.2 & 16.72 & 264.8 & 225.9 & 2.49 & 237.4 & 222.2 \\
\hline 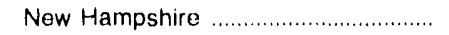 & 46.18 & 172.8 & 177.7 & 14.85 & 226.9 & 186.9 & NA & NA & NA \\
\hline 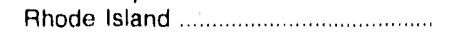 & NA & NA & NA & 17.00 & 270.3 & 224.6 & 2.49 & 241.2 & 208.6 \\
\hline 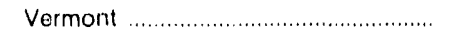 & NA & NA & NA & NA & NA & NA & NA & NA & NA \\
\hline Mirdde Atlantic & 37.03 & 148.9 & 148.8 & 18.61 & 296.1 & 248.9 & 2.44 & 237.4 & 224.0 \\
\hline 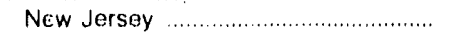 & 46.84 & 175.8 & 171.7 & 18.93 & 303.5 & 251.2 & 2.54 & 245.5 & 224.5 \\
\hline 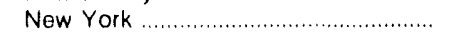 & 40.32 & 157.2 & 158.1 & 18.58 & 295.3 & 248.9 & 2.42 & 235.3 & 223.6 \\
\hline 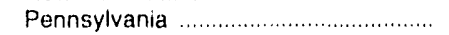 & 35.50 & 144.6 & 145.3 & 18.49 & 293.1 & 245.8 & 3.58 & 348.2 & 333.0 \\
\hline East North Central .................................. & 34.10 & 154.4 & 160.4 & 20.99 & 342.6 & 301.8 & 1.34 & 285.8 & 295.7 \\
\hline 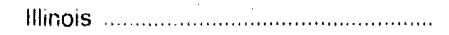 & 38.78 & 181.1 & 190.6 & 22.03 & 353.0 & 312.0 & 3.32 & 326.2 & 326.9 \\
\hline Indiana ...... & 29.08 & 135.9 & 143.2 & 24.27 & 421.8 & 363.4 & 2.70 & 269.5 & 259.1 \\
\hline 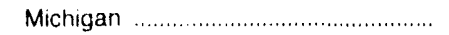 & 38.83 & 172.1 & 175.7 & 19.23 & 315.0 & 2.79 .9 & .19 & 174.8 & 255.5 \\
\hline 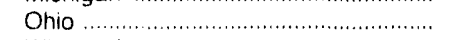 & 35.21 & 148.7 & 152.2 & 21.06 & 345.8 & 311.7 & 3.15 & 313.2 & 346.5 \\
\hline 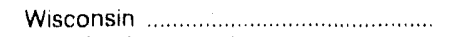 & 28.10 & 144.8 & 146.4 & 23.84 & 410.0 & 364.5 & 3.11 & 309.5 & 325.2 \\
\hline 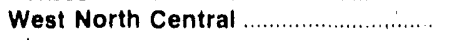 & 19.85 & 114.4 & 115.4 & 22.04 & 369.0 & 276.9 & 2.07 & 211.5 & 210.9 \\
\hline lowa & 21.77 & 121.8 & 123.5 & 25.87 & 445.1 & 372.1 & 2.69 & 267.4 & 202.7 \\
\hline 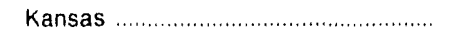 & 22.00 & 123.9 & 123.9 & 25.24 & 432.8 & 318.6 & 1.92 & 197.6 & 206.0 \\
\hline 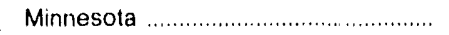 & 21.01 & 119.8 & 119.6 & 25.50 & 441.6 & 369.2 & 2.16 & 214.6 & 198.4 \\
\hline 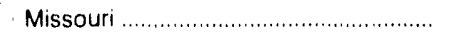 & 28.00 & 135.0 & 138.2 & 19.82 & 323.5 & 237.0 & 2.50 & 245.8 & 277.0 \\
\hline 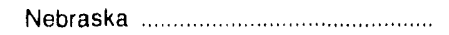 & 14.32 & 82.7 & 84.1 & 15.47 & 248.9 & 241.7 & 2.26 & 236.1 & 268.6 \\
\hline North Dekota & 9.13 & 69.4 & 70.3 & 24.53 & 421.9 & 362.1 & 4.61 & 439.1 & 428.6 \\
\hline South Dakota & 15.24 & 124.1 & 121.4 & 26.47 & 450.2 & 401.7 & NA & NA & NA \\
\hline South Atlantic & ${ }^{\text {A }} 41.19$ & R 165.8 & 166.5 & 17.47 & 277.4 & 227.7 & 2.52 & 248.0 & 212.2 \\
\hline 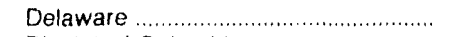 & 46.41 & 179.3 & 180.6 & 17.54 & 277.4 & 232.1 & 2.73 & 254.4 & 242.1 \\
\hline District of Columbia .................................. & NA & NA & NA & 20.78 & 345.6 & 278.9 & NA & NA & NA \\
\hline Florida & 44.20 & 178.7 & 177.7 & 17.19 & 271.1 & 2.21 .3 & 2.49 & 246.2 & 210.1 \\
\hline 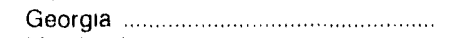 & ค 42.96 & R 177.5 & 174.7 & 24.95 & 426.7 & 283.8 & 3.23 & 315.6 & 273.4 \\
\hline 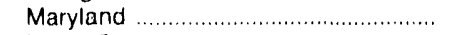 & 40.92 & 161.1 & 157.6 & 17.39 & 276.2 & 236.1 & 2.72 & 260.1 & 259.7 \\
\hline 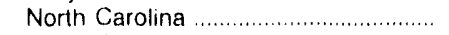 & 44.35 & 177.0 & 177.5 & 23.17 & 398.9 & 348.5 & NA & NA & NA \\
\hline South Carolina & 43.08 & 170.7 & 176.0 & 24.71 & 426.3 & 373.6 & 2.27 & 222.1 & 180.6 \\
\hline 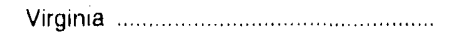 & 39.29 & 154.8 & 155.1 & 17.48 & 284.4 & 236.3 & 2.58 & 247.0 & 204.9 \\
\hline 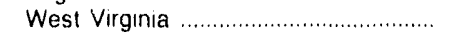 & 35.33 & 142.5 & 144.1 & 26.33 & 450.5 & 395.8 & 4.59 & 458.8 & 389.2 \\
\hline East South Central ................................ & 33.73 & 142.9 & 148.4 & 19.06 & 308.0 & 340.2 & 1.90 & 185.6 & 186.3 \\
\hline 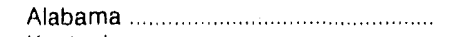 & 44.84 & 186.5 & 194.7 & 23.87 & 414.3 & 366.8 & 2.27 & 22.5 & 207.1 \\
\hline$\ldots \ldots \ldots \ldots \ldots \ldots \ldots \ldots \ldots \ldots \ldots \ldots \ldots \ldots \ldots \ldots \ldots \ldots$ & 26.13 & 113.7 & 119.8 & 27.71 & 475.2 & 384.5 & 2.73 & 267.3 & 246.0 \\
\hline 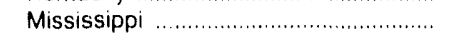 & 42.29 & 167.1 & 181.2 & 16.91 & 267.6 & 327.7 & 1.88 & 183.4 & 184.7 \\
\hline 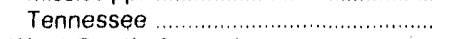 & 31.94 & 134.3 & 133.5 & 22.92 & 394.7 & 350.9 & NA & NA & NA \\
\hline West South Central & 22.69 & 147.5 & 149.5 & 22.54 & 375.0 & 345.5 & 2.24 & 215.7 & 207.8 \\
\hline 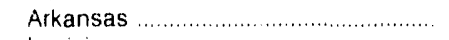 & 28.45 & 163.1 & 158.5 & 22.44 & 370.6 & 435.4 & 1.69 & 165.4 & 135.0 \\
\hline 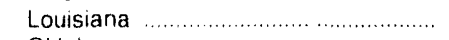 & 26.64 & 162.7 & 155.5 & 23.86 & 379.4 & 347.6 & 1.78 & 170.2 & 163.1 \\
\hline 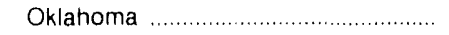 & 24.03 & 136.1 & 148.2 & 23.92 & 415.0 & 268.2 & 3.05 & 292.5 & 281.3 \\
\hline 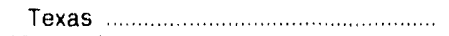 & 21.12 & 144.9 & 147.2 & 22.22 & 373.8 & 329.1 & 2.23 & 215.3 & 209.0 \\
\hline Mountain & 21.80 & 111.6 & 109.5 & 20.79 & 342.6 & 289.4 & 2.24 & 217.2 & 229.4 \\
\hline Arizona & 28.81 & 136.0 & 140.5 & 23.92 & 403.1 & 407.0 & 2.32 & 224.4 & 224.1 \\
\hline 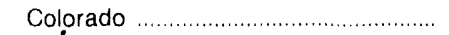 & 20.95 & 106.4 & 106.6 & 23.60 & 409.9 & 384.8 & 2.23 & 228.3 & 228.7 \\
\hline Idaho & NA & NA & NA & NA & NA & NA & NA & NA & NA \\
\hline 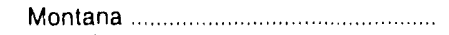 & 9.81 & 57.7 & 54.5 & 25.52 & 430.9 & 403.3 & 1.38 & $: 13.8$ & 126.3 \\
\hline 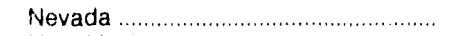 & 33.80 & 152.0 & 136.3 & 17.85 & 286.5 & 244.8 & 2.17 & 211.3 & 271.8 \\
\hline 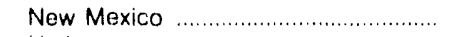 & 22.61 & 123.8 & 117.5 & 22.17 & 378.3 & 308.0 & 2.20 & 213.3 & 213.6 \\
\hline 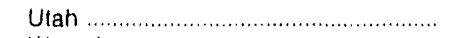 & 28.05 & 123.9 & 126.5 & 26.18 & 445.2 & 398.4 & NA & NA & NA \\
\hline 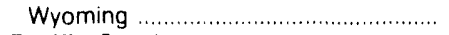 & 14.86 & 84.5 & 84.0 & 24.27 & 415.4 & 379.7 & 3.61 & 348.1 & 367.9 \\
\hline Pacific Contiguous $\ldots \ldots \ldots \ldots \ldots \ldots \ldots \ldots \ldots \ldots$ & 25.40 & 155.6 & 154.5 & 18.93 & 307.9 & 266.0 & 3.04 & 292.4 & 283.1 \\
\hline California & NA & NA & NA & 18.91 & 307.6 & 265.8 & 3.04 & 292.4 & 283.1 \\
\hline 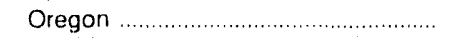 & NA & NA & 139.0 & 26.59 & 452.8 & NA & NA & NA & NA \\
\hline Washington & 25.40 & 155.6 & 155.2 & 20.66 & 336.7 & 403.2 & NA & NA & NA \\
\hline Pacific Noncontiguous ........................... & NA & NA & NA & 20.55 & 329.1 & 286.7 & NA & NA & NA \\
\hline Alaska & NA & NA & NA & NA & NA & NA & NA & NA & NA \\
\hline 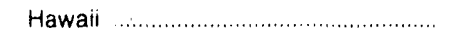 & NA & NA & NA & 20.55 & 329.1 & 286.7 & NA & NA & NA \\
\hline (2) & ค 30.15 & ค 144.6 & 146.6 & 18.17 & 289.3 & 243.9 & 2.42 & 235.5 & 226.3 \\
\hline
\end{tabular}

' Does not include petroleum coke. Petroleum coke cost in 1989 was 85.7 cents per million Btu and in 1988 was 97.2 cents per million Btu. Mcf ... thousand cubic feet.

NA .. Not applicable

A. Revised data.

Notes:-Data are for plants with a fossil-fueled steam-electric nameplate capacity of 50 or more megawatts. $\bullet$ Totals may not equal sum of components because of independent rounding

Source: Federal Energy Regulatory Commission, FERC Form 423, "Monthly Report of Cost and Quality of Fuels for Electric Plants." 


\section{Retail Sales and Revenue}

Electric power is an integral part of our way of life. It is indispensable to the factory, the commercial establishment, the home, and even most recreational facilities. The unavailability of electric power causes inconvenience to customers, loss of industrial production, and could even cause danger to individuals and social disorder. Consequently, the volume of electricity sales and the associated revenue from those sales play an important role in the U.S. economy. This chapter provides summary statistics on the sale of electricity to ultimate consumers, its associated revenue, and the average revenue per kilowatthour sold at the national, Census, and State levels.

\section{Background}

Because electricity itself cannot be stored, it must be generated, transmitted to the consumer, and consumed instantaneously. Electric companies were formed to provide these services. An electric system consists of energy conversion plants (stations) that convert different forms of energy to electric power; transformers to raise the voltage in order to reduce losses in transmitting the power; transmission lines to transmit the power to the general vicinity of consumption; transformers to lower the voltage; and distribution lines to distribute the power to the ultimate consumers. The entire system of generating stations, transformers, transmission lines, distribution lines, etc., is a power system. Power companies plan, design, build, and operate this power system. Some companies may only operate part of a power system. For example, many small power companies only distribute power to ultimate consumers, buying their power from other companies that generate power. Power systems are interconnected for mutual operating and economic advantages.

Electric utilities are high-investment businesses and historically have been treated as monopolies because duplicate facilities, particularly transmission and distribution lines, would be inefficient. Thus, franchises are granted to electric utilities for given geographical areas by regulatory officials. To obrain a franchise, electric utilities must provide service to all consumers in their territories at a reasonable cost.

The service territory of an electric utility is usually composed of many different combinations of consumers. Electric utilities classify their consumers by different factors such as demand, rate schedule, Standard
Industrial Classification (SIC) code, distribution voltage, accounting methods, end-use applications, and other social and economic characteristics. Electric utilities use consumer classifications for planning purposes (load growth, peak demands) and for deriving their rate schedules, often with the approval of a government regulatory agency.

\section{End-Use Sectors}

Consumers within the service territory of an electric utility are generally grouped into end-use sectors -. residential, commercial, and industrial. The categorization of consumer is determined by the electric utility based on its service territory, size, location, ownership, and regulatory structure.

The residential sector includes electricity supplied to private households and apartment buildings, where energy is consumed primarily for space heating, water heating, air conditioning, lighting, refrigeration, cooking, and clothes drying.

The commercial sector may include electricity supplied to nonmanufacturing business establishments, including hotels, motels, restaurants, wholesale businesses, and retail stores; and, health, social, and educational institutions. However, electric utilities may classify commercial service as that to all consumers whose demand or annual usage fall within a specified range. The range may be set by the electric utility based on its rate schedule.

The industrial sector includes electricity supplied to manufacturing, construction, mining, agriculture, fishing, and forestry establishments -- Standard Industrial Classification (SIC) codes 1-39. However, electric utilities may classify industrial service based on demand or annual usage exceeding a specified range $A s$ in the commercial sector, the range may be set by the electric utility based on its rate schedule.

Other sales include public street and highway lighting, railroads and railways, municipalities, divisions or agencies of State and Federal governments under special contracts or agreements, and other utility departments as defined by the pertinent regulatory agency and/or electric utility. 


\section{Revenue Requirements}

The revenue requirements of an electric utility are set to reimburse the utility for providing electric service. The revenue requirement is the anticipated cost of providing services for some period of time in the future, usually a year. Revenue requirements are based on operating expenses, depreciation expense, taxes, and return on the ratebase (profit of the electric utility). The process of determining electricity prices generally follows three stages: 1 ) identification of revenue requirements: 2) allocation of the requirements for different classes of service (sectors); and, 3) establishment of rate schedules for each sector.

The rate schedules developed to generate revenue requirements for electric utilities are unique to each utility. The authority for the approval of these rates is based on the ownership class applicable to the utility. For example, investor-owned electric utilities are regulated by State public service commissions and the Federal Energy Regulatory Commission. Public electric utilities, in most States, are controlled through locally elected or appointed officials. A more detailed discussion on utility classes of ownership is contained in the "Industry Profile" section in the first chapter of this publication.

A rate schedule is a statement that the utility will provide service to a particalar class of consumer at a certain price. Prices between sectors vary based on various objectives of the utility. These objectives include the need to allocate the various costs incurred in providing service, to maintain the existing consumer hase of the utility, and to promote new business.

\section{Average Revenue per Kilowatthour}

A verage revenue per unit (kilowathour) of electricity sold is calculated by dividing total annual revenue by total annual retail sales for each sector and State. Average revenue per kilowatthour from residential consumers are generally higher than for any other sector due in part to the higher costs associated with serving many consumers who use relatively small amounts of electricity. These costs include direct-load costs (such as those for distribution lines, transformers, and meters) in addition to consumer or administrative costs. The industrial sector, which generally covers consumers with the highest use of electricity, has the lowest average revenue per kilowatthour because of the econo. mies of serving a few consumers who use relatively large amounts of electricity.

Because of the type and avalability of capacity, and the cost of fuel, average revenue of electricity sold differ across U. S. Census divisions. The New England and Middle Allantic Census Divisions tend to have average revenue per kilowathour that is higher than the average in the United States becatuse of their higher cost of fuel (petroleum), and their higher labor costs whereas the East North Central and South Atlantic Census Divisions had lower average revenue per kilowatthour because of their reliance on less expensive coal-fired generation. Petroleum is generally a more expensive energy source than coal. Because the Mountain and Pacific Contiguous Census Divisions rely on less expensive hydroelectric generation, average revenue per kilowatthour in these regions is usually below the national average for all divisions. The Census divisions where Federal hydroelectric facilities provide electricity also tend to have lower average revenue per kilowatthour.

\section{Data Sources}

Summary statistics on retail sales of electricity and average revenue are provided in the following tables. These data were obtained from the Form ElA-861, "Annual Electric Utility Report." The Form EIA-861 is used to collect annual data from all electric utilities (approximately 3,250), that own and/or operate facilities within the United States, its territories, and Puerto Rico, for the generation, transmission, distribution, or sate of electric energy primarily for use by the public. More detailed statistics are published in the Electric Sales. Revenue and Bills. ${ }^{24}$

Form EIA-861 figures shown in this publication differ some what from the sum of the monthly Form EIA-826 figures for sales published in the Electric Power Monthly. The statistics from the Form EIA-826 are monthly estimates collected from a sample of electric utilities. Both forms use sectoral classifications (residential, commercial, and industrial) based on the usual classification of consumers by the utilities. The sectoral classification scheme for electricity sales can vary from utility to utility, as influenced by the local public utility commission. At the national level, the Form EIA-861 and Form EIA-826 figures correspond closely (within 3 peicent) for all end-use sectors. Some larger differences occur at the State level particularly for Tennessee due to a misclassification of commercial and industrial data in the Form EIA-826 survey.

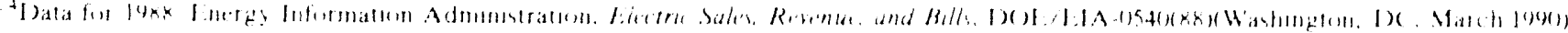

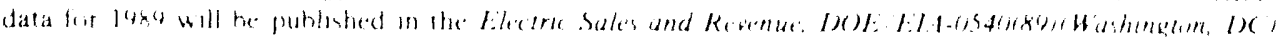


Table 25. Sales to Ultimate Consumers and Assoclated Revenue by Sector, 1985-1989

\begin{tabular}{|c|c|c|c|c|c|}
\hline Item & $1989^{\prime}$ & 1988 & 1987 & 1986 & 1985 \\
\hline \multicolumn{6}{|l|}{ Sales (million kllowatthours) ${ }^{2}$} \\
\hline Residential ............................... & 905,525 & 892,866 & 850,410 & 819,088 & 793,934 \\
\hline 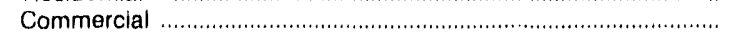 & 725,861 & 699,100 & 660,433 & 630,520 & 605,989 \\
\hline Industrial . & 925,659 & 896,498 & 858,233 & 830,531 & 836,772 \\
\hline 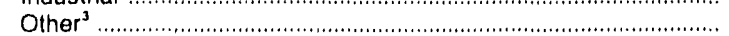 & 89,765 & 89,598 & 88,196 & 88,615 & 87,279 \\
\hline U.S. Total & $2,646,809$ & $2,578,062$ & $2,457,272$ & $2,368,753$ & $2,323,974$ \\
\hline \multicolumn{6}{|l|}{ Revenue (million dollars) } \\
\hline Hesidential ............................ & 69,240 & 66,790 & 63,318 & 60,773 & 58,710 \\
\hline 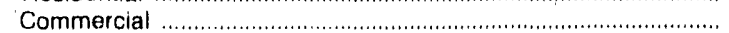 & 52,228 & 49,224 & 46,787 & 45,386 & 44,082 \\
\hline Industrial & 43,719 & 42,145 & 40,949 & 40,982 & 41,580 \\
\hline Other $^{3} \ldots \ldots \ldots \ldots$ & 5,609 & 5,551 & 5,479 & 5,412 & 5,312 \\
\hline 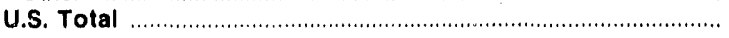 & 170,797 & 163,710 & 156,532 & 152,553 & 149,684 \\
\hline
\end{tabular}

Data for 1989 are preliminary.
The Form ElA-861 data shown in this publication differ somewhat from Form ElA-826, "Monthly Electric Sales and Revenue Report with State Distributions," data published in the Electric Power Monthly and issues of this publication prior to 1988. See Appendix C for a detailed description of these differences.

3 Includes public street and highway lighting, other sales to public authorities, sales to railroads and railways, and interdepartmental sales. Note: Totals may not equal sum of components because of independent rounding.

Source: Energy Information Administration, Form ElA-861, "Annual Electric Utility Report."

Table 26. Average Revenue per Kilowatthour by Sector, 1985-1989

(Cents)

\begin{tabular}{|c|c|c|c|c|c|}
\hline Sector & $1989^{\prime}$ & 1988 & 1987 & 1986 & 1985 \\
\hline (1) & 7.6 & 7.5 & 7.4 & 7.4 & 7.4 \\
\hline 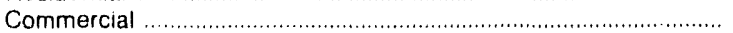 & 7.2 & 7.0 & 7.1 & 7.2 & 7.3 \\
\hline Industrial & 4.7 & 4.7 & 4.8 & 4.9 & 5.0 \\
\hline Other ${ }^{2}$ & 6.2 & 6.2 & 6.2 & 6.1 & 6.1 \\
\hline U.S. Average & 6.5 & 6.4 & 6.4 & 6.4 & 6.4 \\
\hline
\end{tabular}

- Data for 1989 are preliminary.

- Includes public street and highway lighting, other sales to public authorities, sales to railroads and railways, and interdepartmental sales.

Notes: -The average revenue per kllowatthour of electricity sold is calculated by dividing revenue by sales. -Totais may not equal sum of components because of independent rounding. -The Form ElA.861 data shown in this publication differ somewhat from Form ElA-826, "Monthly Electric Sales and Revenue Report with State Distributions," data published in the Electric Power Monthly and issues of this publication prior to 1988. Appendix $C$ for a detailed description of these differences.

Source: Energy Information Administration, Form ElA-861, "Annual Electric Utility Report." 
Table 27. Sales to Ultimate Consumers by Sector, Census Division, and State, 1989 and 1988 (Million Kilowatthours)

\begin{tabular}{|c|c|c|c|c|c|c|c|c|c|c|}
\hline \multirow{2}{*}{$\begin{array}{c}\text { Census Division } \\
\text { State }\end{array}$} & \multicolumn{2}{|c|}{ Total } & \multicolumn{2}{|c|}{ Pesidentlal } & \multicolumn{2}{|c|}{ Commercial } & \multicolumn{2}{|c|}{ Industriai } & \multicolumn{2}{|c|}{ Other' } \\
\hline & $1989^{2}$ & 1988 & $1989^{2}$ & 1988 & $1989^{2}$ & 1988 & $1989^{2}$ & 1988 & $1989^{2}$ & 1988 \\
\hline New England & 104,500 & 102,398 & 37,843 & 37,069 & 37,287 & 36,169 & 27,367 & 27,209 & 2,003 & 1,949 \\
\hline 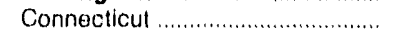 & 27,365 & 26,923 & 10,485 & 10,300 & 10,277 & 9,961 & 6,235 & 6,305 & 367 & 356 \\
\hline 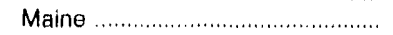 & 11,434 & 11,264 & 4,009 & 3,904 & 2,647 & 2,569 & 4,599 & 4,616 & 179 & 175 \\
\hline Massachusetts …......................... & 45,683 & 44.727 & 15,772 & 15,511 & 18,417 & 17,886 & 10,381 & 10,243 & 1,113 & 1,087 \\
\hline New Hampshire ............................ & 9,086 & 8,848 & 3,542 & 3,464 & 2,020 & 1,947 & 3,420 & 3,339 & 103 & 99 \\
\hline 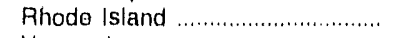 & 6,359 & 6,220 & 2,370 & 2,319 & 2,439 & 2,354 & 1,360 & 1,361 & 190 & 186 \\
\hline 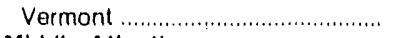 & 4,573 & 4,416 & 1,664 & 1,572 & 1,486 & 1,453 & 1,373 & 1,345 & 50 & 46 \\
\hline 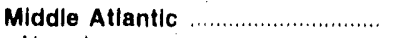 & 305,462 & 300,646 & 96,714 & 95,945 & 101,966 & 99,075 & 93,078 & 92,290 & 13,704 & 13,337 \\
\hline 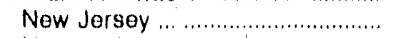 & 63,359 & 62,112 & 20,695 & 20,656 & 26,472 & 25,143 & 15,713 & 15,844 & 478 & 469 \\
\hline 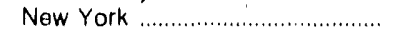 & 128,203 & 125,643 & 37,878 & 37,460 & 47,117 & 46,598 & 31,448 & 30,155 & 11,760 & 11,430 \\
\hline 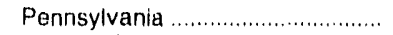 & 113,900 & 112,891 & 38,141 & 37,828 & 28,377 & 27,334 & 45,916 & 46,291 & 1,466 & 1.438 \\
\hline East North Central ......................... & 455,122 & 446,415 & 135,030 & 136,879 & 108,764 & 106,280 & 196,667 & 188,530 & 14,661 & 14,726 \\
\hline 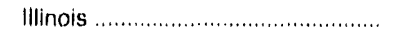 & 109,301 & 110,041 & 32,378 & 33,980 & 30,943 & 30,551 & 38,481 & 37,942 & 7,498 & 7,568 \\
\hline 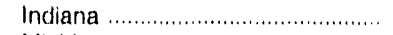 & 72,891 & 71.675 & 22,281 & 22,486 & 15,216 & 15,058 & 34.747 & 33,474 & 647 & 657 \\
\hline 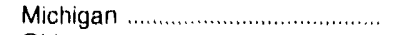 & 82,929 & 82,517 & 25,319 & 25,316 & 20,200 & 19,530 & 36,131 & 36,324 & 1,279 & 1,346 \\
\hline Ohio & 141,624 & 134,316 & 38,792 & 38,713 & 30,019 & 29,004 & 68,314 & 62,238 & 4,499 & 4,360 \\
\hline 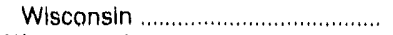 & 48,376 & 47,866 & 16,259 & 16,383 & 12,385 & 12,137 & 18,995 & 18,552 & 738 & 794 \\
\hline West North Central ....................... & 184,036 & P 183,241 & 67,834 & ${ }^{n} 68,918$ & 48,742 & 48,113 & 62,302 & ${ }^{A} 61,155$ & 5,158 & 5,055 \\
\hline 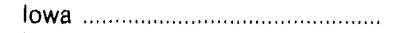 & 28,712 & 28,838 & 10,394 & 10,677 & 6,474 & 6,320 & 11,017 & 11,025 & 827 & 816 \\
\hline 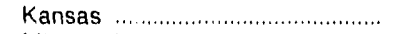 & 25,822 & 25,829 & 8,898 & 9,121 & 8.747 & 8,583 & 7,797 & 7,708 & 380 & 417 \\
\hline Minnesota $\ldots \ldots \ldots \ldots \ldots \ldots$ & 45,932 & 45,728 & 14,778 & 14,996 & 7,707 & 7,792 & 22,700 & 22,131 & 748 & 808 \\
\hline 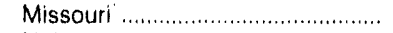 & 52,603 & ${ }^{R} 52,247$ & 21,057 & A 21,348 & 17,916 & ค 17,541 & 12,792 & A 12,556 & 838 & 802 \\
\hline 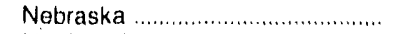 & 17,567 & 17,259 & 6,723 & 6,813 & 5,030 & 4,996 & 4,370 & 4,104 & 1,443 & 1.346 \\
\hline 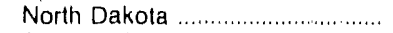 & 7,062 & 7,107 & 3,060 & 3,050 & 1,504 & 1,521 & 2,013 & 2,070 & 484 & 466 \\
\hline 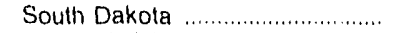 & 6,338 & 6,235 & 2,923 & 2,913 & 1,364 & 1,359 & 1,612 & 1,562 & 438 & 400 \\
\hline 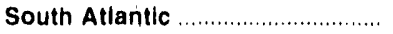 & 520,886 & 499,926 & 206,796 & 199,187 & 146,327 & 139,545 & 151,018 & 145,387 & 16,744 & 15,806 \\
\hline 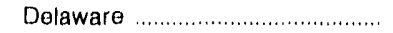 & 8,065 & 7,543 & 2,623 & 2,533 & 2,233 & 2,109 & 3,160 & 2,854 & 49 & 47 \\
\hline District of Columbia ..................... & 9,653 & 9,380 & 1,466 & 1,465 & 4,945 & 4,793 & 2,930 & 2,809 & 312 & 312 \\
\hline 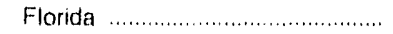 & 138,474 & 130,241 & 68,184 & 63,972 & 48,943 & 45,892 & 17,040 & 16,356 & 4,307 & 4,021 \\
\hline 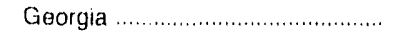 & 77,060 & 74,346 & $2.8,349$ & 27,609 & 21,431 & 19,850 & 26,388 & 25,984 & 891 & 903 \\
\hline 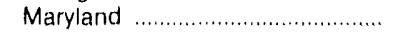 & 49,259 & 47,564 & 19,069 & 18,483 & 10,081 & 11,006 & 19,456 & 17,446 & 652 & 628 \\
\hline North Carolina ................................. & 88,209 & 85,540 & 32,784 & 32,212 & 22,506 & 21,513 & 31,152 & 30,211 & 1,768 & 1,604 \\
\hline 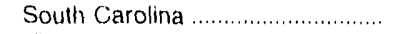 & 53,857 & 52,809 & 17,464 & 17,172 & 11,343 & 10,790 & 24,301 & 24,113 & 749 & 734 \\
\hline 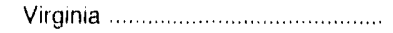 & 73,461 & 70,115 & 29,223 & 28,192 & 19,920 & 18,768 & 16,395 & 15,690 & 7,922 & 7,465 \\
\hline Wesi Virginia ........... & 22,847 & 22,386 & 7,634 & 7,549 & 4,926 & 4,823 & 10,195 & 9,925 & 93 & 91 \\
\hline East South Central & 221,362 & 211,667 & 76,636 & 75,826 & 37,262 & 33,457 & 102,882 & 97,750 & 4,582 & 4,634 \\
\hline 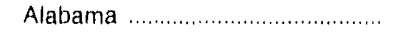 & 58,187 & 56,638 & 19,842 & 19,641 & 10,517 & 9,588 & 27,232 & 26,758 & 596 & 651 \\
\hline 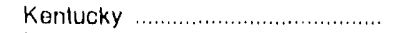 & 58,487 & 54,078 & 16,922 & 16,811 & 8,952 & 8,439 & 30,173 & 26,446 & 2,440 & 2,382 \\
\hline 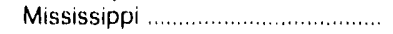 & 29,574 & 28,080 & 11,516 & 11,415 & 6,495 & 5,928 & 10,958 & 10,115 & 605 & 622 \\
\hline 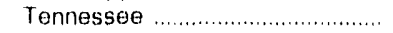 & 75,113 & 72,872 & 28,355 & 27,960 & 11,297 & 9,502 & 34,520 & 34,431 & 940 & 980 \\
\hline West South Central .......................... & 354,594 & 346,955 & 124,174 & 121,809 & 90,343 & 08,168 & 127,978 & 124,788 & 12,099 & 12,189 \\
\hline 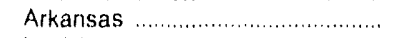 & 26,085 & 25,273 & 9,957 & 9,946 & 5,982 & 5,783 & 9,562 & 8,931 & 584 & 613 \\
\hline 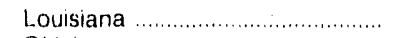 & 61,842 & 60,011 & 20,515 & 20,134 & 13,437 & 13,088 & 24,762 & 23,559 & 3,128 & 3,230 \\
\hline 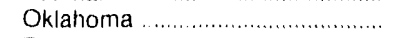 & 37,007 & 37,326 & 14,083 & 14,475 & 9,944 & 10,100 & 11,039 & 10,719 & 1,941 & 2,032 \\
\hline 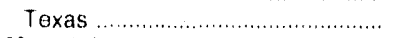 & 229,660 & 224,346 & 79,620 & 77,255 & 60,979 & 59,197 & 82,615 & 81,579 & 6,446 & 6,315 \\
\hline Mountain ............ & 156,305 & 150,269 & 48,527 & 47,309 & 47,999 & 46,269 & 52,461 & 49,820 & 7,318 & 6,870 \\
\hline Arizona & 40,748 & 38,916 & 15,247 & 14,731 & 13,037 & 12,346 & 9,722 & 9,261 & 2,741 & 2,578 \\
\hline 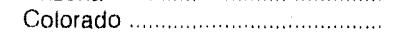 & 30,139 & 29,335 & 9,595 & 9,551 & 13,157 & 12,683 & 6,427 & 6,295 & 959 & 806 \\
\hline 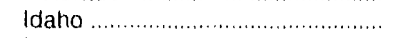 & 17,821 & 17,165 & 5,713 & 5,449 & 4,667 & 4,562 & 7.143 & 6,807 & 297 & 346 \\
\hline 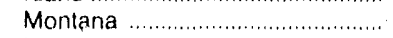 & 13,061 & 12,942 & 3,456 & 3,301 & 2,670 & 2,620 & 6,535 & 6,438 & 400 & 582 \\
\hline 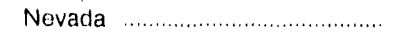 & 14,968 & 13,684 & 5,169 & 4,968 & 3,672 & 3,489 & 5,504 & 4,685 & 624 & 543 \\
\hline 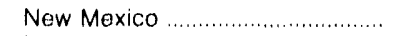 & 13,370 & 12.755 & 3,463 & 3,394 & 4,385 & 4,264 & 4,208 & 4,032 & 1,314 & 1,065 \\
\hline 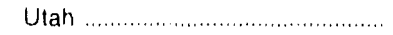 & 14,965 & 14,507 & 4,163 & 4,151 & 4,343 & 4,244 & 5,629 & 5,321 & 830 & 791 \\
\hline 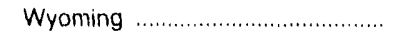 & 11,234 & 10,964 & 1,721 & 1,764 & 2,068 & 2,062 & 7,293 & 6,980 & 151 & 158 \\
\hline 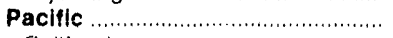 & 332,432 & 324,782 & 108,085 & 106,181 & 103,178 & 98,233 & 107,878 & 105,531 & 13,291 & 14,838 \\
\hline 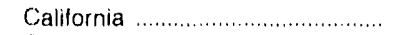 & 204,139 & 200,637 & 64,347 & 64,639 & 75,282 & 70,706 & 55,596 & 54,988 & 8,913 & 10,304 \\
\hline Oregon & 41,619 & 39,312 & 15,085 & 14,338 & 10.942 & 10,605 & 14,913 & 13,633 & 679 & 736 \\
\hline 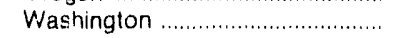 & 86,674 & 84,832 & 28,653 & 27,203 & 16,953 & 16,922 & 37,369 & 36,909 & 3,699 & 3,798 \\
\hline Pacific Noncontiguous .................. & 12,112 & 11,764 & 3,886 & 3,742 & 3,995 & A 3,792 & 4,027 & A 4,037 & 205 & 194 \\
\hline Alaska & 4,142 & ค 4,045 & 1,643 & A 1,590 & 1,897 & ค 1,778 & 450 & 542 & 151 & 136 \\
\hline 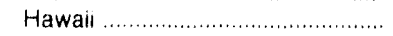 & 7,970 & 7,719 & 2,242 & 2,151 & 2,098 & 2,014 & 3,576 & 3,495 & 54 & 58 \\
\hline U. S. Total & $2,646,809$ & $2,578,062$ & 905,525 & 892,866 & 725,861 & 699,100 & 925,659 & 096,498 & 89,765 & 89,598 \\
\hline
\end{tabular}

' Includes public street and highway lighting, other sales to public authorities, sales to railroads and railways, and interdepartmental sales.

Data for 1989 are preliminary R Ravised data.

Notes: - Totals may not equal sum of components because of independent rounding. -The Form ElA.R61 data shown in this publication differ somewhat from Form EIA-826. "Monthly Electric Sales and Revenue Report with State Distributions," date publish эd in the Electric Power Monthly and issues of this publication prior to 1988. See Appendix $C$ for a detailed description of these differences.

Source: Energy Information Administration, Form EIA-861, "Annual Electric Utility Report." 
Table 28. Number of Ultimate Consumers by Sector, Census Division, and State, 1989 and 1988 (Thousands)

\begin{tabular}{|c|c|c|c|c|c|c|c|c|c|c|}
\hline \multirow{2}{*}{$\begin{array}{c}\text { Census Division } \\
\text { State }\end{array}$} & \multicolumn{2}{|c|}{ Total } & \multicolumn{2}{|c|}{ Residentlal } & \multicolumn{2}{|c|}{ Commercial } & \multicolumn{2}{|c|}{ Industria! } & \multicolumn{2}{|c|}{ Other' } \\
\hline & $1989^{2}$ & 1988 & $1989^{2}$ & 1988 & $1989^{2}$ & 1988 & $1988^{\prime}$ & 1988 & $1909^{2}$ & 1988 \\
\hline 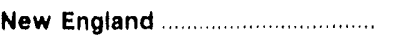 & 5,966 & 5,843 & 5,272 & 5,172 & 622 & 600 & 27 & 26 & 45 & 46 \\
\hline Connecticut ................................. & 1,427 & 1,406 & 1,295 & 1,277 & 123 & 119 & 5 & 5 & 5 & 4 \\
\hline 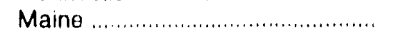 & 647 & 631 & 560 & 547 & 66 & 63 & 3 & 3 & 18 & 18 \\
\hline 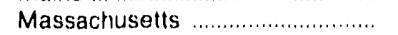 & 2,615 & 2,558 & 2,303 & 2.256 & 285 & 2.75 & 14 & 13 & 13 & 14 \\
\hline New Hampshire .............................. & 558 & $\quad 543$ & 481 & 470 & 70 & 67 & 2 & 2 & 5 & 5 \\
\hline 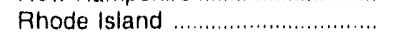 & 429 & 421 & 382 & 375 & 44 & 42 & 3 & 3 & 1 & 2 \\
\hline 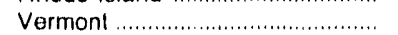 & 289 & 283 & 251 & 246 & 35 & 33 & 1 & 1 & 3 & 3 \\
\hline Middle Atlantic & 15,473 & 15,269 & 13,668 & 13,507 & 1,706 & 1,665 & 52 & 51 & 16 & 45 \\
\hline 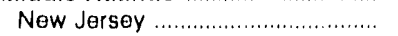 & 3,272 & 3,223 & 2,872 & 2,834 & 379 & 369 & 12 & 12 & 9 & 8 \\
\hline 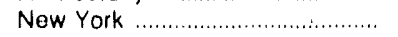 & 7,058 & 6,974 & 6,209 & 6,145 & 809 & 790 & 11 & 10 & 29 & 29 \\
\hline 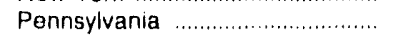 & 5.143 & 5,072 & 4,587 & 4,529 & 518 & 506 & 30 & 29 & 8 & 8 \\
\hline East North Central .......................... & 18,129 & 17,900 & 16,258 & 16,065 & 1,723 & 1,690 & 71 & 69 & 78 & 76 \\
\hline 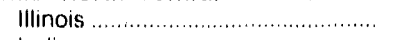 & 4,776 & 4,725 & 4,317 & 4.273 & 435 & 428 & 4 & 4 & 19 & 19 \\
\hline 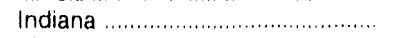 & 2,428 & 2,392 & 2,165 & 2,134 & 237 & 234 & 15 & 14 & 11 & 11 \\
\hline 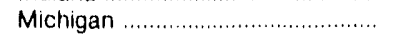 & 4,037 & 3,980 & 3,626 & 3,582 & 378 & 368 & 14 & 15 & 19 & 16 \\
\hline Ohio & 4,660 & 4,603 & 4,164 & 4,117 & 447 & 437 & 31 & 31 & 18 & 18 \\
\hline Wisconsin & 2,229 & 2,199 & 1,987 & 1,959 & 226 & 223 & 6 & 6 & 10 & 12 \\
\hline West North Central ....................... & 8,278 & ${ }^{A} 8,191$ & 7,182 & ค 7,107 & 939 & 926 & 46 & 50 & 112 & 108 \\
\hline lowa & 1,301 & 1,293 & $1,130^{\circ}$ & 1,122 & 156 & 154 & 4 & 4 & 12 & 12 \\
\hline 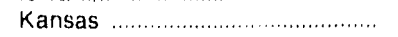 & 1,184 & 1,171 & 1,007 & 994 & 153 & 148 & 13 & 18 & 12 & 11 \\
\hline 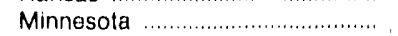 & 1,956 & 1,936 & 1,732 & 1,712 & 194 & 193 & 9 & 8 & 23 & 23 \\
\hline 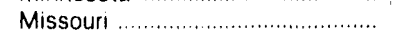 & 2,395 & A 2,360 & 2,120 & ค 2,090 & 250 & 245 & 10 & 10 & 15 & 14 \\
\hline 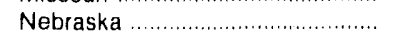 & 795 & 786 & 651 & 646 & 102 & 101 & 5 & 4 & 37 & 34 \\
\hline North Dakota ................................... & 314 & 317 & 263 & 265 & 44 & 44 & 3 & 3 & 4 & 5 \\
\hline South Dakota & 331 & 328 & 279 & 277 & 40 & 40 & 2 & 2 & 9 & $\theta$ \\
\hline South Atlantic & 19,972 & 19,476 & 17,588 & 17,132 & 2,116 & 2,078 & 90 & 86 & 179 & 179 \\
\hline 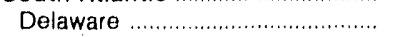 & 307 & 297 & 276 & 267 & 30 & 28 & 1 & 1 & 1 & 1 \\
\hline Districl of Columbia & 216 & 214 & 189 & 187 & 26 & 26 & $\cdot$ & $\cdot$ & - & $\cdot$ \\
\hline 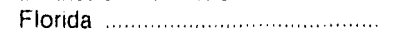 & 6,467 & 6,282 & 5,680 & 5,483 & 688 & 699 & 25 & 26 & 74 & 74 \\
\hline 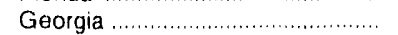 & 2,910 & 2,838 & 2,584 & 2,528 & 288 & 274 & 19 & 20 & 19 & 17 \\
\hline 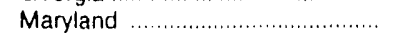 & 1,885 & 1,837 & 1,696 & 1,656 & 177 & 175 & 11 & 6 & 1 & 1 \\
\hline North Carolina .............................. & 3,165 & 3,103 & 2,748 & 2,694 & 375 & 362 & 15 & 15 & 28 & 33 \\
\hline 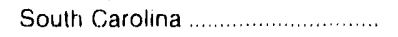 & 1,609 & 1,572 & 1,394 & 1,364 & 194 & 187 & 4 & 4 & 17 & 17 \\
\hline 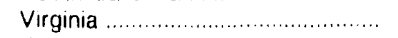 & 2,562 & 2,489 & 2,276 & 2,215 & 244 & 235 & 5 & 5 & 36 & 35 \\
\hline 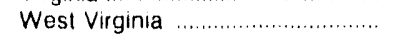 & 851 & 844 & 744 & 739 & 94 & 92 & 10 & 10 & 3 & 3 \\
\hline 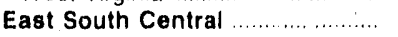 & 6,950 & 6,811 & 6,056 & 5,979 & 774 & 739 & 40 & 43 & 80 & 50 \\
\hline Alabama & 1,876 & 1,840 & 1,639 & 1,616 & 217 & 207 & 9 & 10 & 11 & 7 \\
\hline 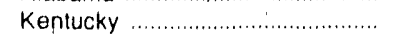 & 1,667 & 1,638 & 1,468 & 1,445 & 174 & 169 & 8 & 9 & 17 & 16 \\
\hline Mississippi & 1,135 & 1,115 & 980 & 971 & 135 & 130 & 6 & 6 & 14 & 8 \\
\hline 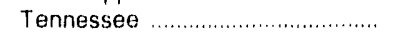 & 2.272 & 2,218 & 1,969 & 1,947 & 248 & 234 & 17 & 18 & 38 & 19 \\
\hline West South Central ........................ & 11,936 & 11,879 & 10,348 & 10,238 & 1,348 & 1,345 & 111 & 147 & 129 & 149 \\
\hline 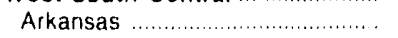 & 1,127 & 1,109 & 978 & 963 & 110 & 108 & 22 & 21 & 18 & 18 \\
\hline 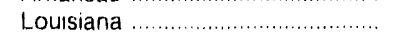 & 1,836 & 1,832 & 1,616 & 1,613 & 188 & 188 & 14 & 15 & 17 & 17 \\
\hline 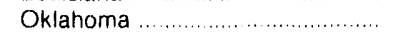 & 1,555 & 1,559 & 1.339 & 1,345 & 186 & 187 & 15 & 15 & 14 & 13 \\
\hline 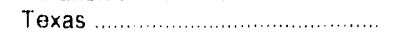 & 7,418 & 7,379 & 6,414 & 6,318 & 863 & 862 & 61 & 96 & 80 & 102 \\
\hline Mountain & 6,093 & 5,977 & 5,267 & 5,162 & 726 & 718 & 29 & 28 & 71 & 69 \\
\hline 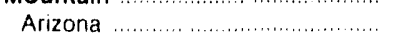 & 1,574 & 1,534 & 1,390 & 1,354 & 158 & 154 & 4 & 4 & 22 & 21 \\
\hline Colorado & 1,595 & 1,572 & 1,378 & 1,363 & 196 & 192 & 2 & 3 & 19 & 14 \\
\hline Idaho & 469 & 464 & 394 & 390 & 68 & 67 & 4 & 4 & 3 & 3 \\
\hline Montana & 408 & 412 & 340 & 339 & 56 & 57 & 3 & 3 & 10 & 13 \\
\hline Nevada & 524 & 489 & 454 & 423 & 68 & 63 & 1 & 1 & 2 & 2 \\
\hline 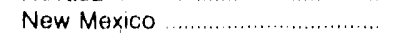 & 661 & 647 & 564 & 551 & 84 & 84 & 5 & 4 & 8 & 8 \\
\hline Utah & 623 & 614 & 554 & 547 & 58 & 57 & 7 & 7 & 4 & 4 \\
\hline 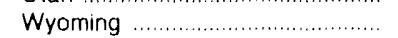 & 239 & 244 & 193 & 194 & 38 & 43 & 3 & 3 & 4 & 4 \\
\hline Pacific Contiguous & 15,252 & 14,906 & 13,336 & 13,008 & 1,787 & 1,769 & 54 & 47 & 74 & 83 \\
\hline California & 11,736 & 11,435 & 10,250 & 9,986 & 1,405 & 1,364 & 38 & 38 & 43 & 47 \\
\hline Oregon & 1,323 & 1,323 & 1,146 & 1,129 & 161 & 181 & 6 & 3 & 10 & 11 \\
\hline Washıngton & 2,193 & 2,148 & 1,941 & 1,892 & 221 & 224 & 10 & 6 & 22 & 25 \\
\hline Pacific Noncontiguous & 587 & ${ }^{1} 576$ & 503 & 493 & 78 & 76 & 1 & 1 & 6 & 5 \\
\hline Alaska & 229 & म 226 & 193 & ค 191 & 31 & 30 & 1 & $i$ & 4 & 4 \\
\hline Hawali ................. & 359 & 351 & 309 & 303 & 47 & 46 & 1 & 1 & 2 & 1 \\
\hline U. S. Average & 108,635 & 106,827 & 95,476 & 93,863 & 11,818 & 11,606 & 522 & 548 & 819 & 811 \\
\hline
\end{tabular}

- Includes public street and higlıway lightıng, other sales to public authoritıes, sales to railroads and railways, and interdepartmental sales

2 Data for 1989 are prelimınary.

- Value less than 0.5 thousand.

A Revisid data

Notes: - Totals may not equal sum of components because of independent rounding. - The number of ultimate consurners is an average of the number of consumers at the close of each rnonth.

Source: Energy Information Administration, Form ElA.861, "Annual Electric Utility Heport." 
Table 29. Revenue from Sales to Ultimate Consumers by Sector, Census Division, and State, 1989 and 1988

(Million Dollars)

\begin{tabular}{|c|c|c|c|c|c|c|c|c|c|c|}
\hline \multirow{2}{*}{$\begin{array}{c}\text { Census Division } \\
\text { State }\end{array}$} & \multicolumn{2}{|c|}{ Total } & \multicolumn{2}{|c|}{ Residential } & \multicolumn{2}{|c|}{ Commerclal } & \multicolumn{2}{|c|}{ Industrial } & \multicolumn{2}{|c|}{ Other' } \\
\hline & $1989^{2}$ & 1988 & $1989^{2}$ & 1988 & $1989^{2}$ & 1988 & $1989^{2}$ & 1988 & $1989^{2}$ & 1988 \\
\hline 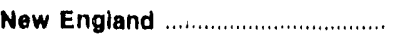 & 8,649 & 8,082 & 3,484 & 3,260 & 3,076 & 2,849 & 1,877 & 1,777 & 213 & 197 \\
\hline 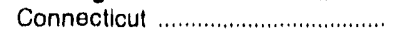 & 2,398 & 2,255 & 1,005 & 950 & 898 & 828 & 449 & 434 & 46 & 43 \\
\hline Maine & 801 & 755 & 341 & 322 & 195 & 182 & 247 & 234 & 18 & 16 \\
\hline 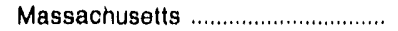 & 3,801 & 3,489 & 1,437 & 1,317 & 1,490 & 1,360 & 758 & 697 & 116 & 106 \\
\hline New Hampshire ............................. & 759 & 732 & 338 & 328 & 177 & 168 & 231 & 224 & 13 & 12 \\
\hline 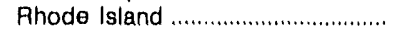 & 526 & 494 & 213 & 200 & 196 & 181 & 102 & 98 & 15 & 15 \\
\hline 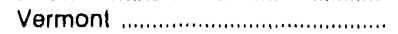 & 365 & 358 & 149 & 143 & 121 & 120 & 90 & 89 & 6 & 5 \\
\hline 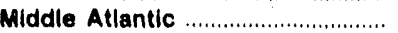 & 25,402 & 24,064 & 8,620 & 9,225 & 9,215 & 8,669 & 5,432 & 5,117 & 1,135 & 1,052 \\
\hline 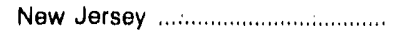 & 5,604 & 5,286 & 2,085 & 2,023 & 2,317 & 2,116 & 1,126 & 1,072 & 77 & 75 \\
\hline 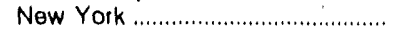 & 11,385 & 10,736 & 4,140 & 3,920 & 4,671 & 4,492 & 1,665 & 1,489 & 909 & 834 \\
\hline 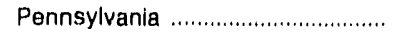 & 8,413 & 8,042 & 3,395 & 3,283 & 2,227 & 2,061 & 2,641 & 2,556 & 149 & 143 \\
\hline East North Central .......................... & 28,511 & 27,872 & 10,788 & 10,817 & 7,819 & 7,466 & 8,917 & 8,620 & 988 & 949 \\
\hline Illinois ........................ & 8,210 & 8,048 & 3,227 & 3,309 & 2,407 & 2,295 & 2,068 & 1,960 & 507 & 483 \\
\hline 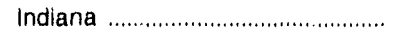 & 3,949 & 4,031 & 1,544 & 1,607 & 932 & 951 & 1,420 & 1,420 & 52 & 53 \\
\hline 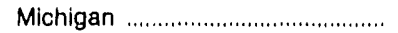 & 5,621 & 5,450 & 1,915 & 1,873 & 1,588 & 1.491 & 2,007 & 1,975 & 112 & 110 \\
\hline Ohio & 8,099 & 7,715 & 3,017 & 2,938 & 2,163 & 2,020 & 2,649 & 2,504 & 270 & 253 \\
\hline 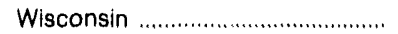 & 2,632 & 2,629 & 1,085 & 1,091 & 729 & 728 & 772 & 760 & 47 & 50 \\
\hline West North Central ............................. & 10,938 & ค 10,952 & 4,809 & ${ }^{A} 4,891$ & 3,078 & H 3,031 & 2,745 & 2,723 & 306 & 305 \\
\hline lowa & 1,695 & 1,731 & 787 & 810 & 414 & 415 & 444 & 457 & 50 & 49 \\
\hline 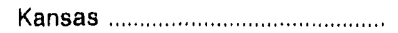 & 1,657 & 1,691 & 682 & 714 & 569 & 560 & 377 & 386 & 28 & 31 \\
\hline 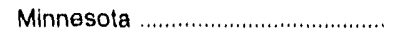 & 2,433 & 2,457 & 994 & 1,014 & 460 & 463 & 931 & 930 & 48 & 51 \\
\hline 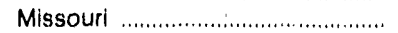 & 3,396 & ค 3,376 & 1,545 & ค 1,565 & 1,160 & 1.136 & 633 & 619 & 58 & 57 \\
\hline 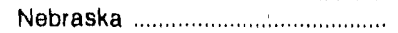 & 972 & 915 & 414 & 405 & 287 & 271 & 184 & 158 & 87 & 80 \\
\hline 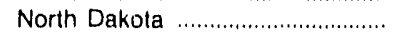 & 403 & 409 & 188 & 190 & 97 & 99 & 100 & 102 & 18 & 10 \\
\hline South Dakota & 383 & 373 & 199 & 195 & 91 & 88 & 76 & 72 & 17 & 18 \\
\hline 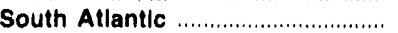 & 32,551 & 30,950 & 15,177 & 14,420 & 9,502 & 9,031 & 6,867 & 6,528 & 1,005 & 971 \\
\hline Delaware & 507 & 502 & 214 & 213 & 149 & 147 & 139 & .138 & 5 & 4 \\
\hline 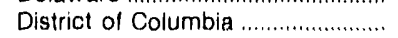 & 567 & 576 & $8 B$ & 92 & 311 & 317 & 150 & 149 & 18 & 18 \\
\hline Florida & 9,660 & 9,197 & 5,273 & 5,001 & 3,236 & 3,091 & 863 & 833 & 289 & 272 \\
\hline Georgia & 4,909 & 4,632 & 2,049 & 1,939 & 1,556 & 1,414 & 1,235 & 1,213 & 69 & 66 \\
\hline 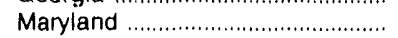 & 2,946 & 2,780 & 1,308 & 1,241 & 668 & 716 & 918 & 772 & 52 & 50 \\
\hline 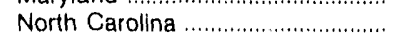 & 5,508 & 5,255 & 2,511 & 2,406 & 1,413 & 1,344 & 1,470 & 1,402 & 114 & 103 \\
\hline South Carolina & 3,039 & 2,955 & 1,256 & 1,224 & 704 & 669 & 1,035 & 1,022 & 43 & 40 \\
\hline 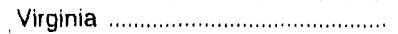 & 4,322 & 3,978 & 2,027 & 1,856 & 1,196 & 1,071 & 690 & 640 & 408 & 410 \\
\hline 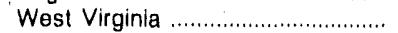 & 1,091 & 1,075 & 451 & 448 & 267 & 262 & 366 & 358 & 8 & 7 \\
\hline East South Central & 11,959 & 11,670 & 4,656 & 4,572 & 2,342 & 2,088 & 4,704 & 4,738 & 256 & 272 \\
\hline Alabama & 3,233 & 3,134 & 1,307 & 1,277 & 712 & 637 & 1,182 & 1,183 & 33 & 37 \\
\hline 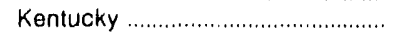 & 2.824 & 2,779 & 953 & 952 & 476 & 453 & 1,283 & 1,263 & 112 & 112 \\
\hline 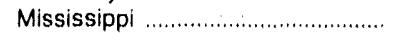 & 1,829 & 1,735 & 786 & 746 & 466 & 422 & 530 & 512 & 47 & 55 \\
\hline 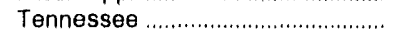 & 4,072 & 4.023 & 1,611 & 1,597 & 689 & 577 & 1,709 & 1,780 & 64 & 68 \\
\hline 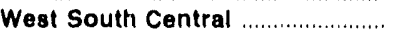 & 20,469 & 19,762 & 8,796 & 8,481 & 5,626 & 5,415 & 5,296 & 5,114 & 751 & 752 \\
\hline 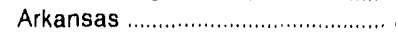 & 1,681 & 1,606 & 774 & 757 & 401 & 376 & 468 & 431 & 39 & 41 \\
\hline 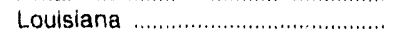 & 3,689 & 3,509 & 1,505 & 1,435 & 940 & 888 & 1,053 & 985 & 190 & 201 \\
\hline Oklahoma & 2,049 & 2,058 & 962 & 975 & 582 & 586 & 404 & 393 & 102 & 104 \\
\hline 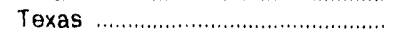 & 13,050 & 12,588 & 5,556 & 5,314 & 3,703 & 3,564 & 3,372 & 3,305 & 420 & 406 \\
\hline 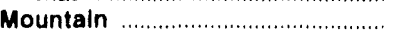 & 9,169 & 8,847 & 3,501 & 3,420 & 3,123 & 3,001 & 2,162 & 2,068 & 383 & 358 \\
\hline 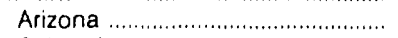 & 3,059 & 2,887 & 1,349 & 1,289 & 1,057 & 995 & 524 & 487 & 130 & 115 \\
\hline 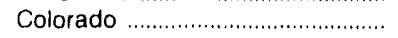 & 1,791 & 1.731 & 673 & 662 & 760 & 722 & 292 & 284 & 67 & 62 \\
\hline Idaho & 674 & 635 & 275 & 258 & 199 & 188 & 187 & 175 & 14 & 14 \\
\hline Montana & 531 & 531 & 186 & 179 & 123 & 124 & 204 & 205 & 18 & 23 \\
\hline Nevada & 788 & 740 & 296 & 298 & 221 & 210 & 244 & 208 & 26 & 23 \\
\hline 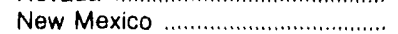 & 976 & 940 & 312 & 306 & 361 & 351 & 223 & 214 & 80 & 69 \\
\hline Utah & 867 & 907 & 308 & 324 & 294 & 298 & 226 & 241 & 39 & 44 \\
\hline 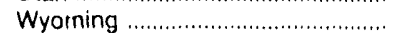 & 483 & 477 & 104 & 103 & 108 & 111 & 261 & 254 & 9 & 9 \\
\hline Pacific Contiguous. & 22,123 & 20,560 & 8,040 & 7,361 & 8,088 & 7,325 & 5,447 & 5,204 & 548 & 671 \\
\hline 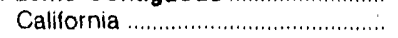 & 17,309 & 15,967 & 6,080 & 5,518 & 6,867 & 6,142 & 3,963 & 3,780 & 400 & 527 \\
\hline 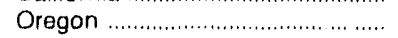 & 1,774 & 1,693 & 719 & 686 & 529 & 515 & 493 & 457 & 33 & 34 \\
\hline Washington & 3,040 & 2,901 & 1,241 & 1,156 & 692 & 668 & 991 & 967 & 116 & 110 \\
\hline Paciflc Noncontlguous ................ & 1,024 & 951 & $\begin{array}{r}1,241 \\
369\end{array}$ & 344 & 359 & 327 & 272 & ค 257 & 24 & 23 \\
\hline Alaska & 382 & 364 & 161 & n 154 & 166 & ค 151 & 35 & ค 40 & 20 & 18 \\
\hline 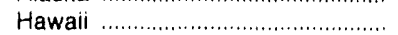 & 642 & 588 & 208 & 190 & 193 & 176 & 237 & 217 & 5 & 5 \\
\hline U. S. Total & 170,797 & 163,710 & 69,240 & 66,790 & 52,228 & 49,224 & 43,719 & 42,145 & 5,609 & 5,551 \\
\hline
\end{tabular}

includes public street and highway lighting, other sales to public authorities, sales to railroads and railways, and interdepartmental sales.

- Data for 1989 are preliminary.

A Revised data.

Note: Totals may not equal sum of components because of independent rounding.

Source: Energy intormation Administration, Form ElA-861, "Annual Electric Utility Report." 
Table 30. Average Revenue per Kilowatthour by Sector, Census Division, and State, 1989 and 1988

(Cents)

\begin{tabular}{|c|c|c|c|c|c|c|c|c|c|c|}
\hline \multirow{2}{*}{$\begin{array}{c}\text { Census Division } \\
\text { State }\end{array}$} & \multicolumn{2}{|c|}{ Total } & \multicolumn{2}{|c|}{ Residential } & \multicolumn{2}{|c|}{ Commerclal } & \multicolumn{2}{|c|}{ liıdustrial } & \multicolumn{2}{|c|}{ Other' } \\
\hline & $1989^{2}$ & 1988 & $1989^{2}$ & 1988 & $1989^{2}$ & 1988 & $1989^{2}$ & 1988 & $1989^{2}$ & 1988 \\
\hline New England & 8.3 & 7.9 & 9.2 & 8.8 & 8.2 & 7.9 & 6.9 & 6.5 & 10.6 & 10.1 \\
\hline 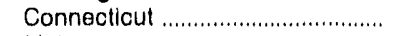 & 8.8 & 8.4 & 9.6 & 9.2 & 8.7 & 8.3 & 7.2 & 6.9 & 12.4 & 12.0 \\
\hline 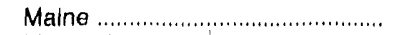 & 7.0 & 6.7 & 8.5 & 8.3 & 7.4 & 7.1 & 5.4 & 5.1 & 10.0 & 9.4 \\
\hline Massachusetts .............................. & 8.3 & 7.8 & 9.1 & 8.5 & 8.1 & 7.7 & 7.3 & 6.8 & 10.4 & 9.7 \\
\hline New Hampshire ............................. & 8.4 & 8,3 & 9.5 & 9.5 & 8.8 & 8.6 & 6.7 & 6.7 & 12.9 & 11.8 \\
\hline 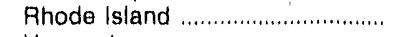 & 8.3 & 7.9 & 9.0 & 8.6 & 8.0 & 7.7 & 7.5 & 7.2 & 7.8 & 8.2 \\
\hline 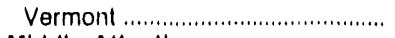 & 8.0 & 8.1 & 8.9 & 9.1 & 8.1 & 8.3 & 6.5 & 6.6 & 11.3 & 10.7 \\
\hline 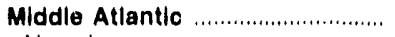 & 0.3 & 8.0 & 9.9 & 0.6 & 9.0 & 8.8 & 5.8 & 5.5 & 8.3 & 7.8 \\
\hline 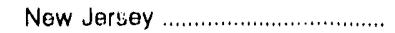 & 8.8 & 8.5 & 10.1 & 9.8 & 8.8 & 8.4 & 7.2 & 6.8 & 16.2 & 16.1 \\
\hline 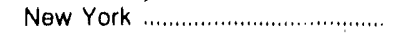 & 8.9 & 8.4 & 10.9 & 10.5 & 9.9 & 9.6 & 5.3 & 4.9 & 7.7 & 7.3 \\
\hline 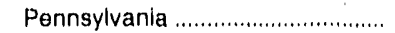 & 7.4 & 7.1 & 8.9 & 8.7 & 7.8 & 7.5 & 5.8 & 5.5 & 10.2 & 9.9 \\
\hline 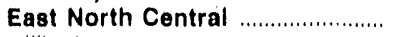 & 6.3 & 6.2 & 8.0 & 7.9 & 7.2 & 7.0 & 4.5 & 4.6 & 6.7 & 6.4 \\
\hline 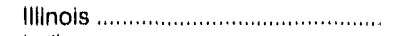 & 7.5 & 7.3 & 10.0 & 9.7 & 7.8 & 7.5 & 5.4 & 5.2 & 6.8 & 6.4 \\
\hline Indlana & 5.4 & 5.6 & 6.9 & 7.1 & 6.1 & 6.3 & 4.1 & 4.2 & 8.0 & 8.1 \\
\hline 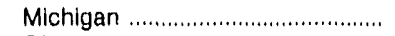 & 6.8 & 6.6 & 7.6 & 7.4 & 7.9 & 7.6 & 5.6 & 5.4 & 8.7 & 8.2 \\
\hline 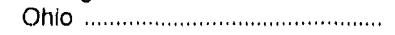 & 5.7 & 5.7 & 7.8 & 7.6 & 7.2 & 7.0 & 3.0 & 4.0 & 6.0 & 5.8 \\
\hline 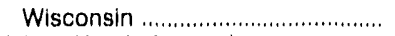 & 5.4 & 5.5 & 6.7 & 6.7 & 5.9 & 6.0 & 4.1 & 4.1 & 6.3 & 6.2 \\
\hline 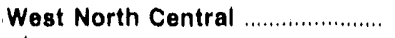 & 5.9 & 6.0 & 7.1 & 7.1 & 6.3 & 6.3 & 4.4 & 4.5 & 5.9 & 6.0 \\
\hline 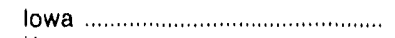 & 5.9 & 6.0 & 7.6 & 7.6 & 6.4 & 6.5 & 4.0 & 4.1 & 6.1 & 6.1 \\
\hline 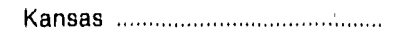 & 6.4 & 6.5 & 7.7 & 7.8 & 6.5 & 6.5 & 4.8 & 5.0 & 7.5 & 7.4 \\
\hline 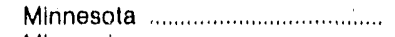 & 5.3 & 5.4 & 6.7 & 6.8 & 6.0 & 5.9 & 4.1 & 4.2 & 6.4 & 6.3 \\
\hline 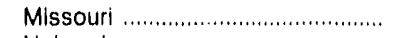 & 6.5 & 6.5 & 7.3 & 7.3 & 6.5 & 6.5 & 4.9 & 4.9 & 6.9 & 7.1 \\
\hline 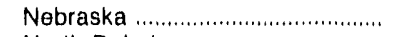 & 5.5 & 5.3 & 6.2 & 5.9 & 5.7 & 5.4 & 4.2 & 3.9 & 6.0 & 6.0 \\
\hline 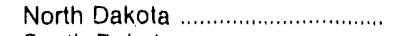 & 5.7 & 5.8 & 6.1 & 6.2 & 6.5 & 6.5 & 4.9 & 4.9 & 3.8 & 4.0 \\
\hline 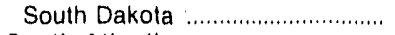 & 6.0 & 6.0 & 6.8 & 6.7 & 6.7 & 6.5 & 4.7 & 4.6 & 3.8 & 4.5 \\
\hline 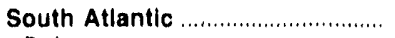 & 6.2 & 6.2 & 7.3 & 7.2 & 6.5 & 6.5 & 4.5 & 4.5 & 6.0 & 6.1 \\
\hline 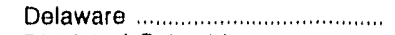 & 6.3 & 6.7 & 8.2 & 8.4 & 6.7 & 7.0 & 4.4 & 4.8 & 9.3 & 9.2 \\
\hline 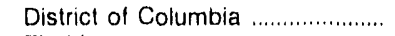 & 5.9 & 6.1 & 6.0 & 6.3 & 6.3 & 6.6 & 5.1 & 5.3 & 5.7 & 5.6 \\
\hline 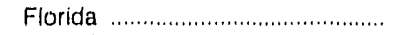 & 7.0 & 7.1 & 7.7 & 7.8 & 6.6 & 6.7 & 5.1 & 5.1 & 6.7 & 6.8 \\
\hline 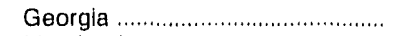 & 6.4 & 6.2 & 7.2 & 7.0 & 7.3 & 7.1 & 4.7 & 4.7 & 7.8 & 7.3 \\
\hline 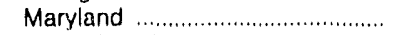 & 6.0 & 5.8 & 6.9 & 6.7 & 6.6 & 6.5 & 4.7 & 4.4 & 7.9 & 7.9 \\
\hline North Carolina ................................ & 6.2 & 6.1 & 7.7 & 7.5 & 6.3 & 6.2 & 4.7 & 4.6 & 6.4 & 6.4 \\
\hline 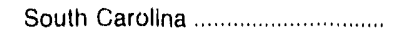 & 5.6 & 5.6 & 7.2 & 7.1 & 6.2 & 6.2 & 4.3 & 4.2 & 5.8 & 5.5 \\
\hline 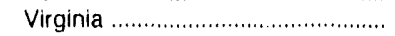 & 5.9 & 5.7 & 6.9 & 6.6 & 6.0 & 5.7 & 4.2 & 4.1 & 5.2 & 5.5 \\
\hline West Virginia & 4.8 & 4.8 & 5.9 & 5.9 & 5.4 & 5.4 & 3.6 & 3.6 & 8.3 & 8.2 \\
\hline 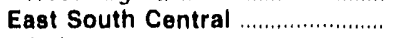 & 5.4 & 5.5 & 6.1 & 6.0 & 6.3 & 6.2 & 4.6 & 4.8 & 5.6 & 5.9 \\
\hline 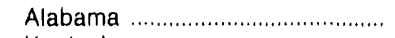 & 5.6 & 5.5 & 6.6 & 6.5 & 6.8 & 6.6 & 4.3 & 4.4 & 5.6 & 5.6 \\
\hline 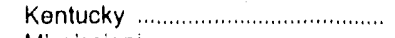 & 4.8 & 5.1 & .5 .6 & 5.7 & 5.3 & 5.4 & 4.3 & 4.8 & 4.6 & 4.7 \\
\hline 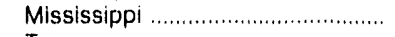 & 6.2 & 6.2 & 6.8 & 6.5 & 7.2 & 7.1 & 4.8 & 5.1 & 7.7 & 8.8 \\
\hline 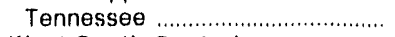 & 5.4 & 5.5 & 5.7 & 5.7 & 6.1 & 6.1 & 5.0 & 5.2 & 6.8 & 7.0 \\
\hline 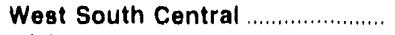 & 5.8 & 5.7 & 7.1 & 7.0 & 6.2 & 6.1 & 4.1 & 4.1 & 6.2 & 6.2 \\
\hline 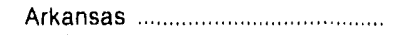 & 6.4 & 6.4 & 7.8 & 7.6 & 6.7 & 6.5 & 4.9 & 4.8 & 6.7 & 6.7 \\
\hline 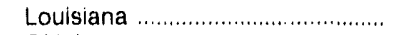 & 6.0 & 5.8 & 7.3 & 7.1 & 7.0 & 6.8 & 4.3 & 4.2 & 6.1 & 6.2 \\
\hline 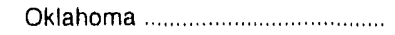 & 5.5 & 5.5 & 6.8 & 6.7 & 5.9 & 5.8 & 3.7 & 3.7 & 5.2 & 5.1 \\
\hline 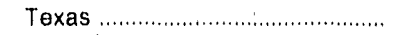 & 5.7 & 5.6 & 7.0 & 6.9 & 6.1 & 6.0 & 4.1 & 4.1 & 6.5 & 6.4 \\
\hline 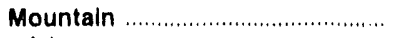 & 5.9 & 5.9 & 7.2 & 7.2 & 6.5 & 6.5 & 4.1 & 4.2 & 6.2 & 5.2 \\
\hline 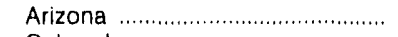 & 7.5 & 7.4 & 8.8 & 8.8 & 8.1 & 8.1 & 5.4 & 5.3 & 4.7 & 4.5 \\
\hline 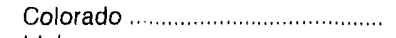 & 5.9 & 5.9 & 7.0 & 6.9 & 5.8 & 5.7 & 4.5 & 4.5 & 7.0 & 7.7 \\
\hline Idaho & 3.8 & 3.7 & 4.8 & 4.7 & 4.3 & 4.1 & 2.6 & 2.6 & 4.6 & 3.9 \\
\hline 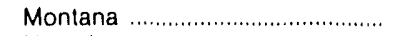 & 4.1 & 4.1 & 5.4 & 5.4 & 4.6 & 4.8 & 3.1 & 3.2 & 4.5 & 3.9 \\
\hline 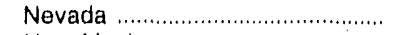 & 5.3 & 5.4 & 5.7 & 6.0 & 6.0 & 6.0 & 4.4 & 4.4 & 4.2 & 4.2 \\
\hline 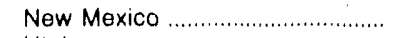 & 7.3 & 7.4 & 9.0 & 9.0 & 8.2 & 8.2 & 5.3 & 5.3 & 6.1 & 6.4 \\
\hline 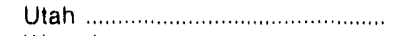 & 5.8 & 6.2 & 7.4 & 7.8 & 6.8 & 7.0 & 4.0 & 4.5 & 4.6 & 5.5 \\
\hline 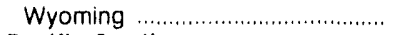 & 4.3 & 4.4 & 6.0 & 5.8 & 5.2 & 5.4 & 3.6 & 3.6 & 6.0 & 5.6 \\
\hline 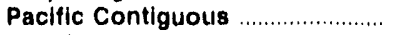 & 6.7 & 6.3 & 7.4 & 6.9 & 7.8 & 7.5 & 5.0 & 4.9 & 4.1 & 4.5 \\
\hline 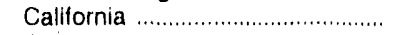 & 8.5 & 8.0 & 9.4 & 8.5 & 9.1 & 8.7 & 7.1 & 6.9 & 4.5 & 5.1 \\
\hline 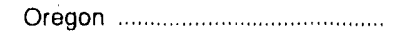 & 4.3 & 4.3 & 4.8 & 4.8 & 4.8 & 4.9 & 3.3 & 3.4 & 4.8 & 4.6 \\
\hline 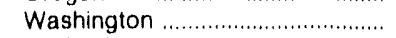 & 3.5 & 3.4 & 4.3 & 4.2 & 4.1 & 3.9 & 2.7 & 2.6 & 3.1 & 2.9 \\
\hline Paciflc Noncontiguous ................ & 8.5 & 8.1 & 9.5 & ${ }^{n} 9.2$ & 9.0 & 8.6 & 6.7 & 6.4 & 11.9 & 11.9 \\
\hline Alaska & 9.2 & ค 9.0 & 9.8 & ค 9.7 & 8.8 & 8.5 & 7.7 & 7.5 & 13.1 & 13.6 \\
\hline 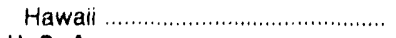 & 8.1 & 7.6 & 9.3 & 8.8 & 9.2 & 8.8 & 6.6 & 6.2 & 8.5 & 8.0 \\
\hline 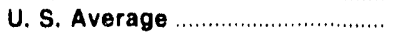 & 6.5 & 6.4 & 7.6 & 7.5 & 7.2 & 7.0 & 4.7 & 4.7 & 6.2 & 6.2 \\
\hline
\end{tabular}

1 Includes public street and highway lighting, other sales to public authorities, sales to railroads and railways, and interdepartmental sales. The average revenue for other sales may include ownership, operation, maintenance, and rental fees for equipinent and/or demand and service charges.

2 Data for 1989 are preliminary.

A Revised data.

Notes: -The average sevenue per kilowathour of electricity sold is calculated by dividing reveriue by sales. $\bullet$ Totals may not equal sum of components because of independent rounding. -The Form EIA-861 data shown in this publication differ somewhat from Form EIA-826, "Monthly Electric Sales and Revenue Report with State Distributions," data published in the Electric Power Monthly and issues of this publication prior to 1988. Sae Appendix $C$ for a detailed descriplion of these differences.

Source: Energy Information Administration, Form ElA-861, "Annual Electric Utility Report." 


\section{Financial Statistics}

This chapter presents data on the financial results of operations for selected investor-owned and publicly owned electric utilities. Composite financial data on other segments of the eleciric utility industry, for example, Federal electric utilities and rural electric cooperatives, are not included in this discussion. ${ }^{25}$ The statistical data exhibited consist of the Composite Statement of Income, the Compositc Balance Sheet, Financial Indicators, and Revenue and Expense Statistics. Historical data are provided for a 3-year period on the investor-owned electric utilities and for 1 year on the publicly owned electric utilities.

\section{Background}

Today, virtually all investor-owned electric utilities are subject to State and Federal regulatory jurisdiction. State commissions have the authority to regulate electric rates of companies engaged in providing service to ultimate consumers (retail sales of electricity) and to oversee the issuance of mortgage bonds, debentures, notes, preferred stocks, and common stock. The Federal Energy Regulatory Commission (FERC) regulates, among other things, electric rates for interstate wholesale transactions. The ratemaking process is one that sets rates at levels that cover all operating expenses and taxes with a remaining balance that will enable a utility to pay a fair return on funds invested by the stockholders.

A component of any economic regulatory activity is the determination of financing and accounting rules. As a consequence of regulatory jurisdiction, regulations for financing and accounting are more critical to the electric power industry than to most other nonregulated industries. Both FERC and State commissions normally use quasi-judicial proceedings for financial and accounting regulation.

Many of the publicly owned electric utilities are selfregulated (for example, the City of Dover, Delaware), while some fall under the jurisdiction of the public utility commission within the State(s) where they provide electricity to ultimate consumers (such as, in the State of (Ohio). Because of the absence of any requirement for reporting to a specific regulatory body, the accounting practices and policies of publicly owned electric utilities vary greatly. Many publicly owned electric utilities use the FERC Uniform System of Accounts or variations of this (and other) accounting systems. As a result, the composite statistics provided must be viewed with an appropriate degree of caution.

Because of the respondent burden in preparing this information, publicly owned electric utilities are permitted to use the year-end period on which their fiscal practices are based. Electric utilities must submit data for a 12-month period (does not necessarily end on December 31) and show consistency in their methods and reporting dates. Data are provided for a subset of the selected publicly owned electric utilities with the fiscal years ending June 30, and December 31, 1989.

\section{Composite Statement of Income}

This statement provides a summary of the revenue collected from consumers in return for services rendered within the reporting period; summarizes (in broad categories) the costs incurred by the electric utility in the production and delivery of electricity; and reports the net income or profit that remains for the owners of the business. Because of the somewhat unique nature of regulated electric utilities, the income statement that is standard to other nonregulated industries has been recast to reflect the reporting conventions in the electric power industry. For example, accounting for capital used in construction requires additional reporting on the income statement because of the perpetual nature of construction work in progress. Also, on occasion, electric utilities are required to defer the recovery of certain costs and earnings from consumers until a future period. This introduces additional accounting requirements, which must be reflected on all the financial statements.

\section{Composite Balance Sheet}

A summary of plant, property, and cash held by the electric utilities, as well as the receivables of the electric utilities, are represented as assets on the composite balance sheet. Future funds obligated by the electric util- 
ities to acquire assets are shown as liabilities and any increased investment by stockholders is shown as capital on the balance sheet. The standard balance sheet used in the electric power industry emphasizes capital intensity while the balance sheet used by nonregulated industries emphasizes liquidity. The balance sheet represents an accounting at a particular time. For this section, the composite balance sheets are presented for investor-owned electric utilities at the end of a calendar year and for publicly owned electric utilities at the fiscal years ending June 30, and December 31, 1989.

\section{Composite Financial Indicators}

The financial statement accounts presented in this chapter represent compiled statistics resulting from the activity of the selected electric utilities. The measurement of how well the electric utility industry performs in different areas can be approximated by comparing some of the asset and income accounts to other relevant accounts. Using the financial statement information, some basic indicators that can be used to analyze or assess the financial condition of the industry are provided. The method used to derive these selected financial indicators is ratio analysis.

\section{Investor-Owned Electric Utilities}

Activity ratios evaluate how assets of the investorowned electric utilities are managed. The electric utility industry is one of the most capital intensive industries in the United States and activity ratios are paramount indicators of the magnitude of their capital intensity. These ratios demonstrate the financial relationship, which exists between the assets and revenue, sales, and, income that these fixed and total assets generate. The ratios on electric-fixed-asset (not plant) turnover and total-asset turnover assess the effective and efficient use of assets in the generation of income.

Leverage ratios summarize the overall debt burden and debt structure of the investor-owned electric utilities. In addition, these ratios indicate the financial ability to meet debt service requirements and how well management uses leverage to increase the value of the investment of the stockbolders. The financial soundness of an industry is directly related to the ability of the industry to raise capital and to be able 10 provide a reasonuble return of and on the capital invested. In order to measure the ability to do this, a number of indicators are used. Current assets to current liabilities is a measure of liquidity. For example, do the investor-owned electric utilities have sufficient cash and other assets (current), which can be quickly converted to cash to cover maturing obligation (current liabilities)? Long-term debt to capitalization, preferred stock to capitalization, and common-stuck equity to capitalization portray the financial structure and highlight the extent to which debt and other fixed obligations are used to finance operations. Total debt to total assets shows the amount of debt that has been incurred in relationship to the total assets possessed. As the value of this ratio in. creases, the financial risks also become grealer and more apparent. Con:mon-stock equity to total assets evaluates financial strength. As net worth increases in relationship to tocal assess, the debt portion is decreased and financial risks are lowered. Interest coverage before taxes without AFUDC (Allowance for Funds Used During Construction), a noncash source of incorne, is an indicator of the ability of the investor-owned electric utility to ensure its payment of annual interest costs and maintain its credit ratings.

Profitability ratios indicate operatung effectiveness of the investor-owned electric utilities and are used to further evaluate the management of income. The profit margin is equal to net income divided by revenue. This ratio is used extensively and represents the overall measure of income performance. Return on averagecommon-stock equity measures the rate of return on equity capital invested. Since one of the main objectives of management is to earn the highest return permissible, this ratio is the best single measure of the effectiveness of management from the perspective of the stockholders. Return on investment measures the overall rate of return that has been earned on assets. This ratio, which is determined by dividing total assets into net income, provides an indicator of the overall financial performance.

\section{Publicly Owned Electric Utilities}

Ratios are provided on the publicly owned electric utilites to assist in understanding the financial performance of the publicly owned segment of the industry. Six ratios calculated from the statement of income and four ratios calculated from the balance sheet are pro. vided. Electric utility plant per dollar of revenue highlights the capital intensity of the utility. Current assets to current liabilities provides a measure of the ease by which the utility can meet its current obligations. Electric utility plant as a percent of total assets represents the total gross investment in electric plant divided by the total assets of the utility. A significant variation in this ratio should signal a relatively fundamental change in the activities of the utility. Net electric utility plant as a percent of total assets represents the remaining book value and a significant variation should signal a change for the utility. Accumulated provision for depreciation as a percent of total electric plant measures the cost of recovery of the use of the assets over a period of time for a utility; an increase indicates the asset life is being used up. The ratios of electric operating and maintenances expenses, electric depreciation and amortization, taxes and lax equivalents, contributions and services, and interest on long-term debt to electric operating revenue are indicators of how resources were used to produce income. 


\section{Revenue and Expense Statistics}

These summary statistics are basic in any analysis of the operating soundness of an electric utility. In order to conduct this analysis, it is necessary to separate the electric utility revenue and expense information from other utility revenue and expense data. Emphasis is placed on total electric operating expenses. Data are presented so that operating costs are separate from maintenance, depreciation, and taxes. For comparative purposes, the ratio of income from utility operations is included, although ratios of electric utility operating expenses are emphasized.

\section{Data Sources}

The financial statistics reported in this chapter on the investor-owned electric utilities are compiled from data extracted from the FERC Form 1, "Annual Report of Electric Utilities, Licensees and Others." This form is a restricted-universe census used annually to collect detailed accounting, financial, and operating data from selected investor-owned electric utilities having, in each of the last 3 consecutive years, sales or transmission service that exceeds any one or more of the following:

- 1 militon megawatthours of total sales

- 100 megawathours of sales for resale

- 50) megawatthours of gross interchange out or

- 500 megawatthours of wheeling for others (deliveries plus losses).
Approximately 180 investor-owned electric utilities are required to submit the FERC Form 1. These major investor-owned electric utilities represent about twom thirds of all investor-owned electric utilities. The electric utilities are required to follow the Uniform System of Accounts prescribed by the FERC (in cooperation with the National Association of Regulatory Utilit; Commissioners). More detailed financial statistics on investor-owned electric utilities are published in the Financial Statistics of Selected Investor-Owned Electric Utilities: ${ }^{26}$

The financial statistics on the publicly owned electric utilities are compiled from data extracted from the Form ElA-412, "Annual Report of Public Electric Utilities." This form is a restricted-universe census used annually to collect detailed accounting, financial, and operating data from selected publicly owned electric utilities having, in each of the last 2 consecutive years, sales that exceed any one of the following:

- 120,000 megawatthours of sales to ultimate consumers or

- 120,000 megawatthours of sales for resale.

Approximately 450 publicly owned electric utilities are required to submit the Form EIA-412. These selected publicly owned electric utilities represent about one-fourth of all publicly owned electric utilities and over 80 percent of total sales by publicly owned electric utilities to ultimate consumers. These electric utilities are requested, but not required, to follow the FERC Uniform System of Accounts. More detailed financial statistics on public electric utilities, Federal electric utilities, and rural electric cooperatives are published in the Financial Statistics of Selected Publicly Owned Electric Utilities. ${ }^{27}$

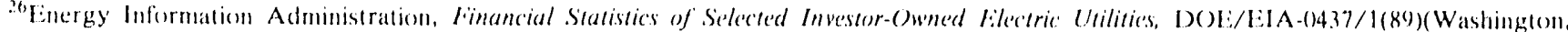

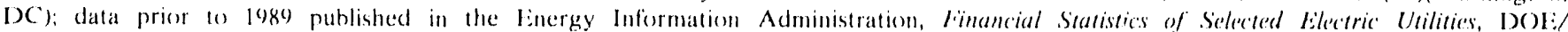
EIA -() $437(8,8)($ Washinglom, I)(., February 1900$)$

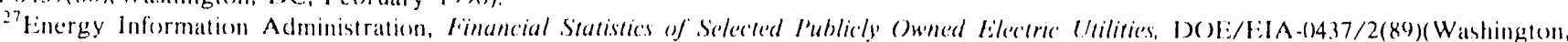

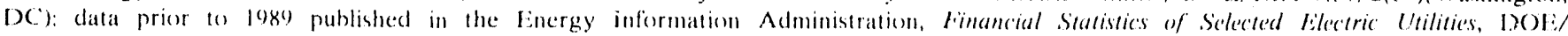
EIA.(1)4.37(88)(Washington, DC: Fehruary 190(0)
} 
Table 31. Composite Statement of Income for Selected Investor-Owned Electric Utilities, 1987-1989

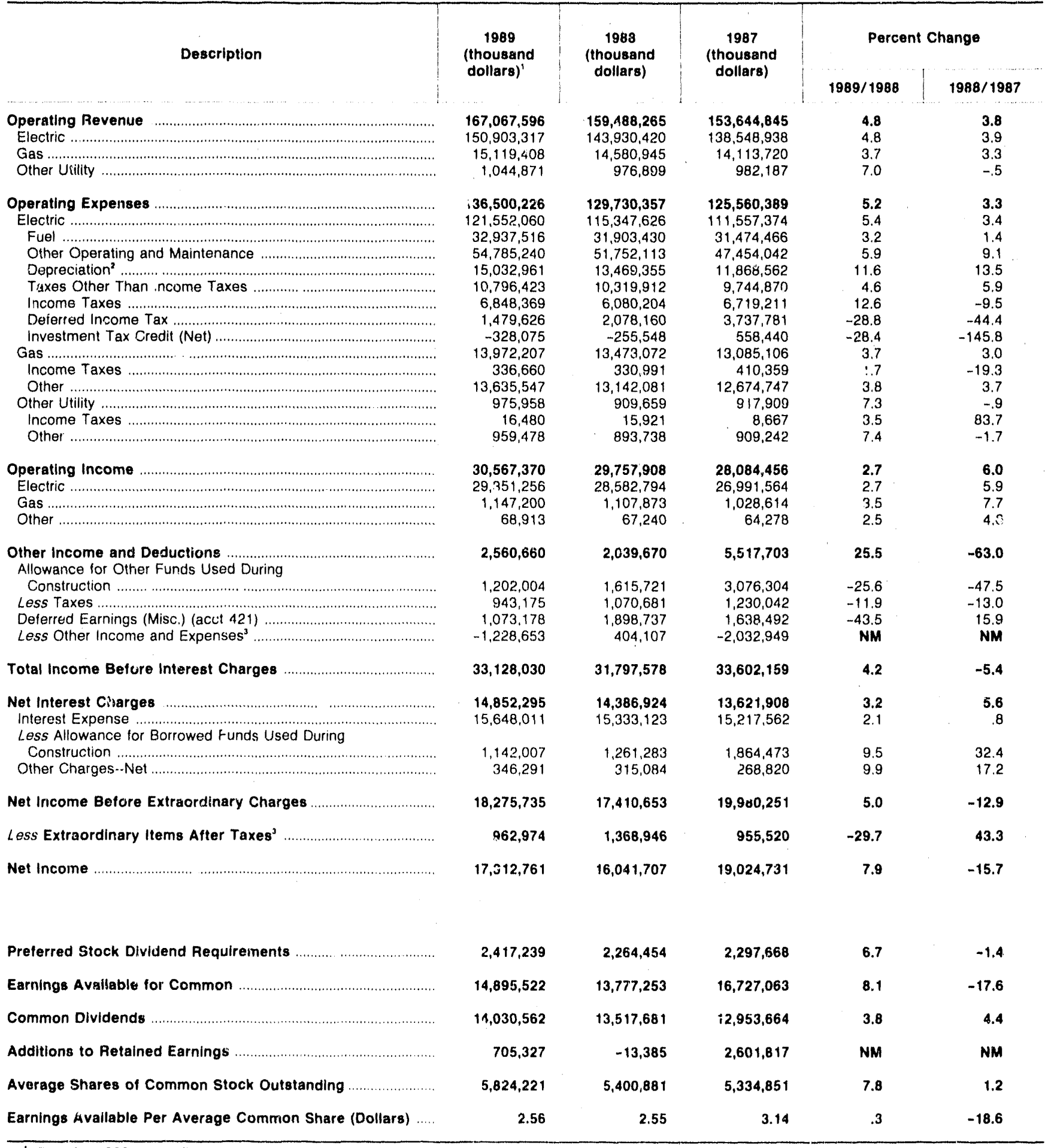

\footnotetext{
Data for 1989 are preliminary

Includes amortization and depletion

3 Other income and txpenses and Extraordinary tiems After Taxes were affected negatively by aftertax write offs, accounting adjustments, and regulatory rate decisions. The majority of the charges were directly related to the the treatment of nuclear plants

NM $:$ Not meaningful.

Notes: - Totals may not equal sum of components because of independent rounding. Percent change is calculated before rounding.

Source: Federal Energy Regulatory Commission. FFAC Form 1. "Arnual Report of Major Electic Ulitities, Licensees and Others." See Appendix C for a detailed description of this restricted-universe census.
} 
Table 32. Composite Balance Sheet for Selected Investor-Owned Electric Utillties, 1987-1989

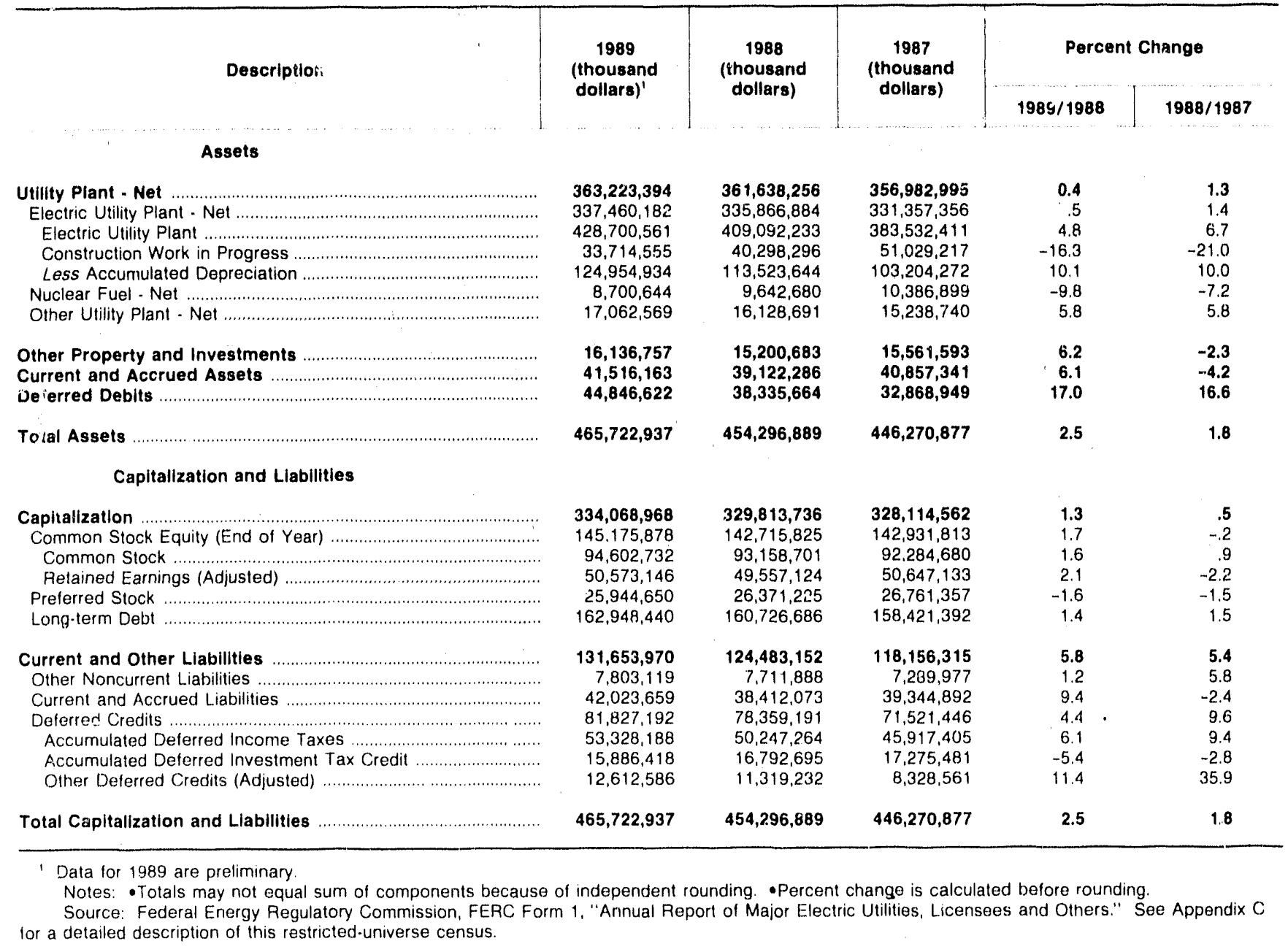

Figure 21. The Revenue Dollar from Operations for Selected Investor-Owned Electric Utilities, 1989

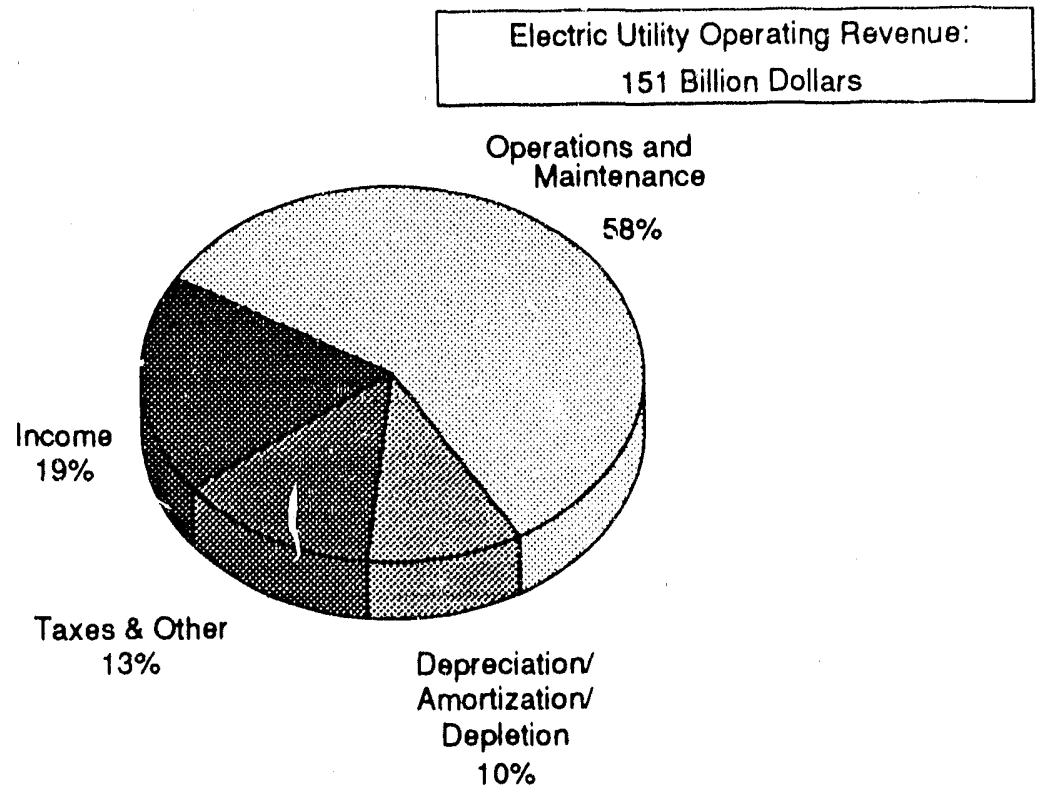

Note: Totals may not equal sum of components because of independent rounding.

Source: Federa! Energy Regulatory Commission. FERC Form 1. "Annual Report of Major Electric Utilities, Licensees and Others." See Appendix $\mathrm{C}$ for a detailed description of this restricted-universe census. 
Table 33. Composite Financial Indicators for Selected Investor-Owned Electric Utilities, 1987-1989

\begin{tabular}{|c|c|c|c|}
\hline Description' & $1989^{2}$ & 1988 & 1987 \\
\hline \multicolumn{4}{|l|}{ Activity } \\
\hline 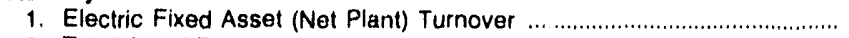 & 0.45 & 0.43 & 0.42 \\
\hline 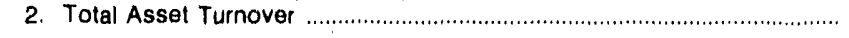 & .36 & .35 & .34 \\
\hline \multicolumn{4}{|l|}{ Leverage } \\
\hline 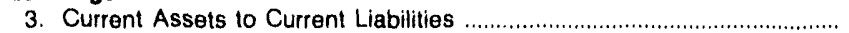 & .99 & 1.02 & 1.04 \\
\hline 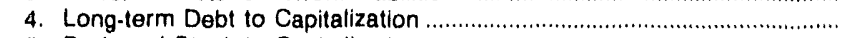 & 48.78 & 48.73 & 48.28 \\
\hline 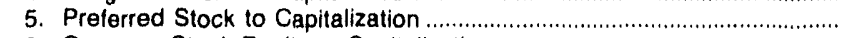 & 7.77 & B.00 & 8.16 \\
\hline 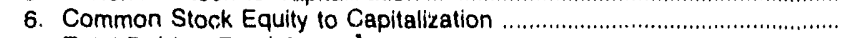 & 43.46 & 43.27 & 43.56 \\
\hline 7. Total Debt to Total Assets ${ }^{3}$ & 36.26 & 36.67 & 37.18 \\
\hline 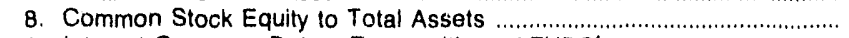 & 31.17 & 31.42 & 32.03 \\
\hline 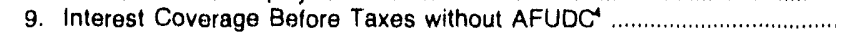 & 2.46 & 2.35 & 2.42 \\
\hline \multicolumn{4}{|l|}{ Profitability } \\
\hline 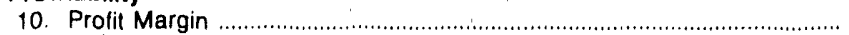 & 10.36 & 10.06 & 12.38 \\
\hline 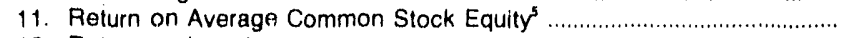 & 12.03 & 11.23 & 13.49 \\
\hline 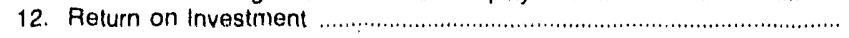 & 3.72 & 3.53 & 4.26 \\
\hline
\end{tabular}

- Indicalors $1,2,3$, and 9 are ratios. Indicators 4 through 8 and 10 through 12 are percentages.

2 Data for 1989 are preliminary.

3 Total debt is the sum of Long-term Debt and Short-term Debt. The values for Shor-term Debt included in Current and Accrued Liabilities (Notes Payable) were $\$ 5,928,421$ for $1989 ; \$ 5,843,202$ for 1988 ; and $\$ 7,500,551$ for 1987

- Taxes Other Than Income Taxes (included in Other Income and Expenses. Net) should be adced to Taxes from Other Income and Deductions. The values were $\$ 251,243$ for $1989 ; \$ 552,805$ for 1988 ; and $\$ 399,495$ for 1987 .

5 The Average Common Stock Equity is the average of the beginning and ending year balances. The values for the beginning of each year were $\$ 142,715,825$ for $1989 ; \$ 142,931,813$ for 1988 ; and $\$ 139,050,743$ for 1987

AFUDC = Allowance for Funds Used Cisring Construction.

Notes: - Formulas for computing the financial indicators are in Appendix C. - Indicators 4, 5 , and 6 may not sum lo 100 percent because of independent rounding.

Source: Federal Energy Regulatory Commission, FERC Form 1, "Annual Report of Major Electric Utilities, Licensees and Others." See Appendix $C$ for a detailed description of this restricted-universe census.

Figure 22. Share of Electric Operation Expenses for Selected Investor-Owned Electric Utilities, 1989

Electric Operation Expenses:

76 Billion Dollars

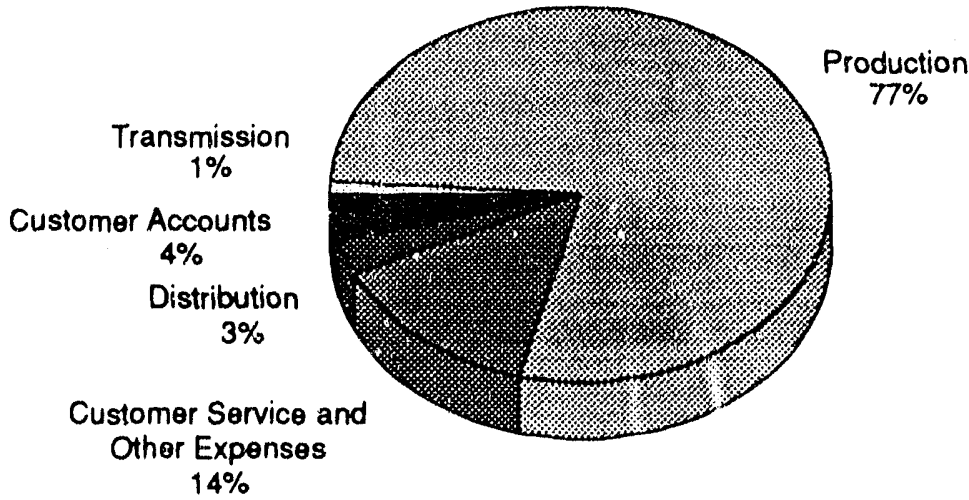

Note: Totals may not equal sum of components because of independent rounding.

Source: Federal Energy Regulatory Commission, FERC Form 1. "Annual Report of Major Electric Utilities, Licensees and Others." See Appendix C for a detailed description of this restricted-universe census. 
Table 34. Revenue and Expense Statistics for Selected Investor-Owned Electric Utilities, 1987-1989

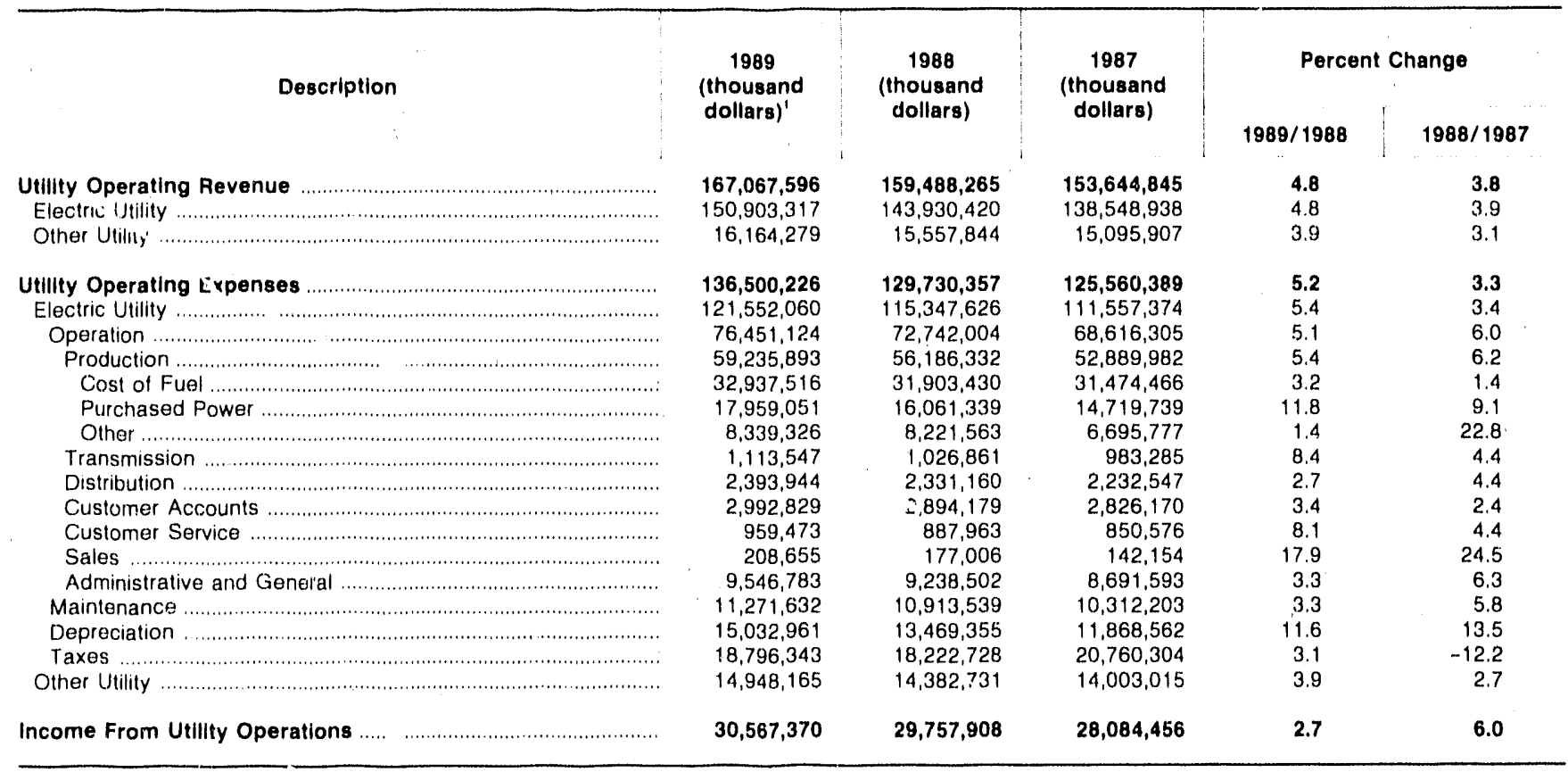

1 Data for 1989 are preliminary.

Notes: - Totals may not equal sum of components because of independent rounding. •Percent change is calculated before rounding.

Source: Federal Energy Regulatory Commission, FERC Form 1, "Annual Report of Major Electric Utilities, Licensees and Others." See Appendix C for a detailed description of this restricted-universe census.

Table 35. Revenue and Expense Percentages for Selected Investor-Owned Electric Utilities, 1987-1989

\begin{tabular}{|c|c|c|c|}
\hline Description & $1989^{\prime}$ & 1988 & 1987 \\
\hline Utillty Operating Revenue & 100.0 & 100.0 & 100.0 \\
\hline Electric Utility & 90.3 & 90.2 & 90.2 \\
\hline 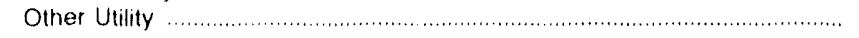 & 9.7 & 9.8 & 9.8 \\
\hline Utility Operating Expenses & 81.7 & 81.3 & 81.7 \\
\hline Electric Utılity ....................... & 72.8 & 72.3 & 72.6 \\
\hline 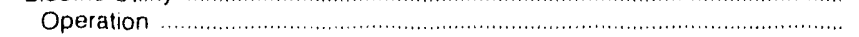 & 45.8 & 45.6 & 44.7 \\
\hline 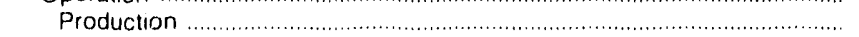 & 35.5 & 35.2 & 34.4 \\
\hline Cost of Fuel & 19.7 & 20.0 & 20.5 \\
\hline 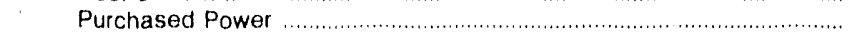 & 10.7 & 10.1 & 9.6 \\
\hline Other & 5.0 & 5.2 & 4.4 \\
\hline 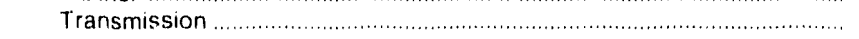 & .7 & .6 & 6 \\
\hline 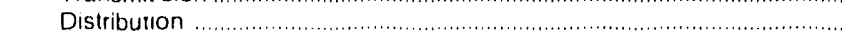 & 1.4 & 1.5 & 1.5 \\
\hline Customer Accounts & 1.8 & 1.8 & 1.8 \\
\hline Customer Service & 6 & .6 & .6 \\
\hline 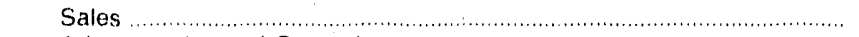 & .1 & .1 & .1 \\
\hline 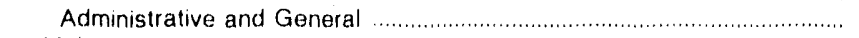 & 5.7 & 5.8 & 5.7 \\
\hline 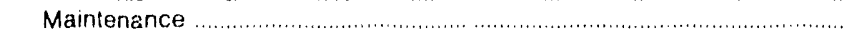 & 6.7 & 6.8 & 6.7 \\
\hline 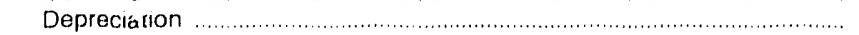 & 9.0 & 8.4 & 7.7 \\
\hline Taxes & 11.3 & 11.4 & 13.5 \\
\hline Other Utility & 8.9 & 9.0 & 9.1 \\
\hline Income From Utility Operations & 18.3 & 18.7 & 18.3 \\
\hline
\end{tabular}

1 Data for 1989 are preliminary.

Note: Totals may not equal sum of components because of independent rounding.

Source: Federal Energy Regulatory Commission, FERC Form 1, "Annual Report of Major Electric Utilities, Licensees and Others." See Appendix $\mathrm{C}$ lor a detailed description of this restricted-universe census. 
Table 36. Composite Statement of Income for Selected Publicly Owned Electric Utilities, 1989 (Thousand Dollars)

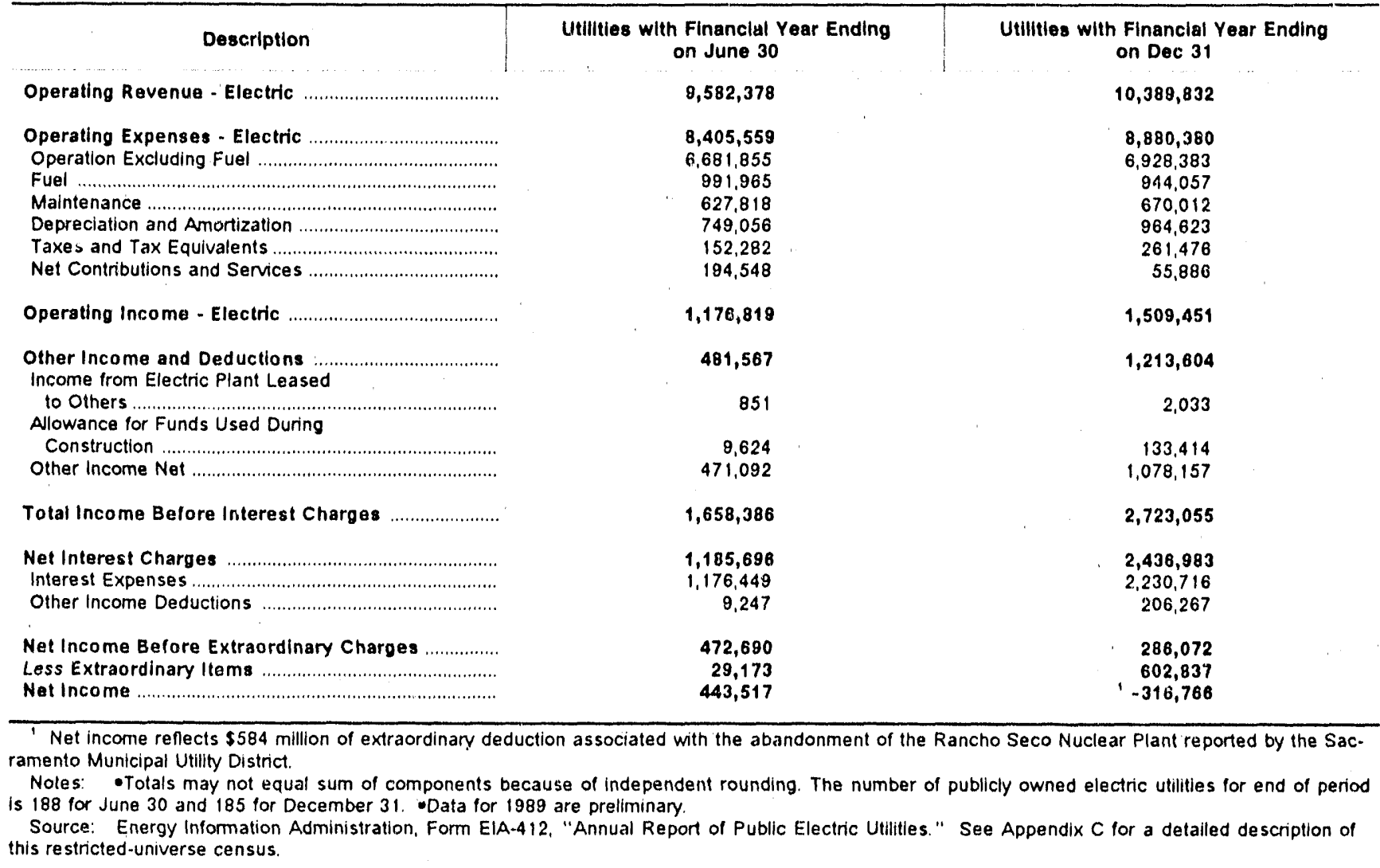

Table 37. Composite Balance Sheet for Selected Publicly Owned Electric Utilities, 1989 (Thousand Dollars)

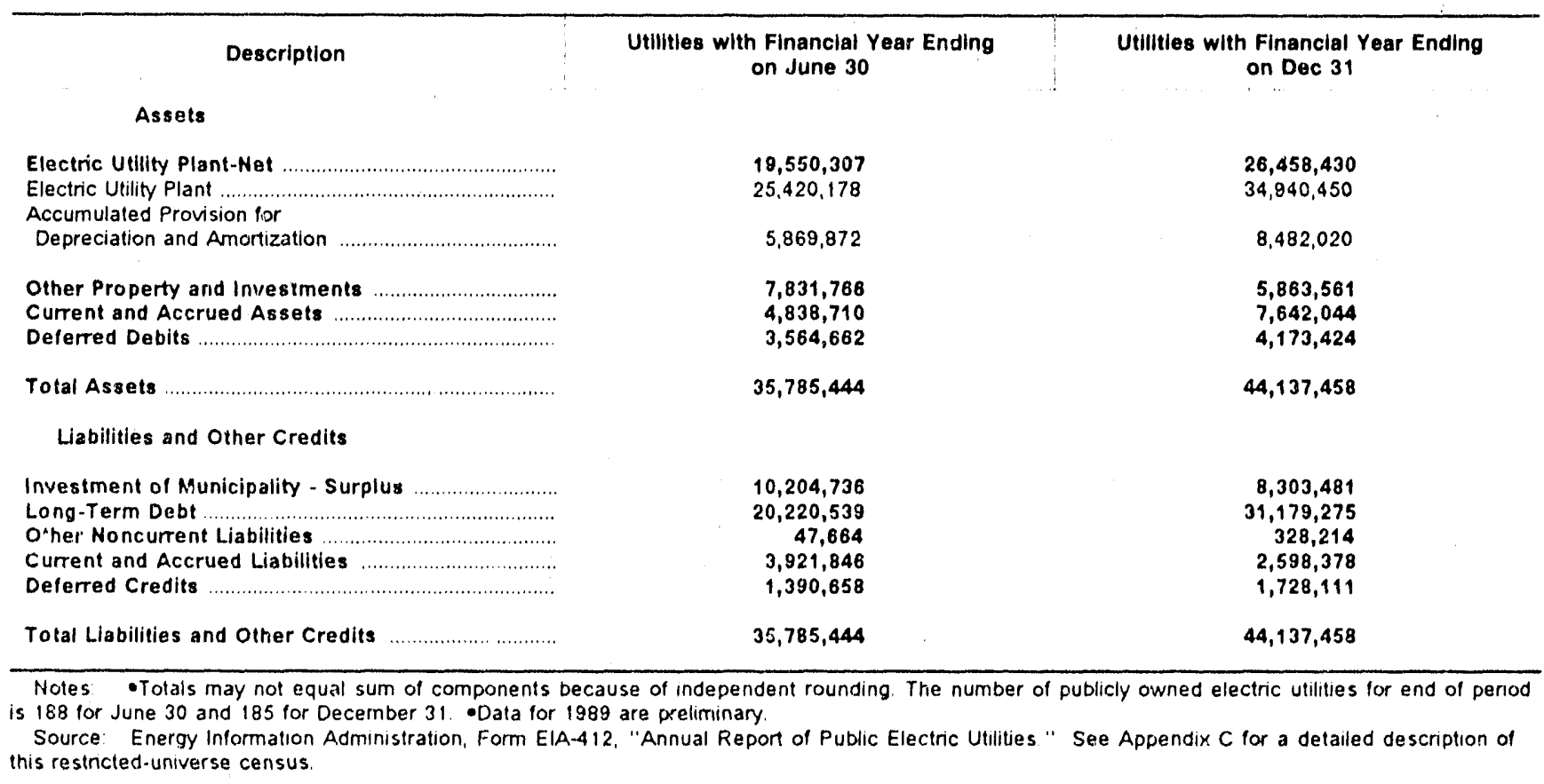


Table 38. Composite Financial Indicators for Selected Publicly Owned Electric Utilities, 1989 (Thousand Dollars)

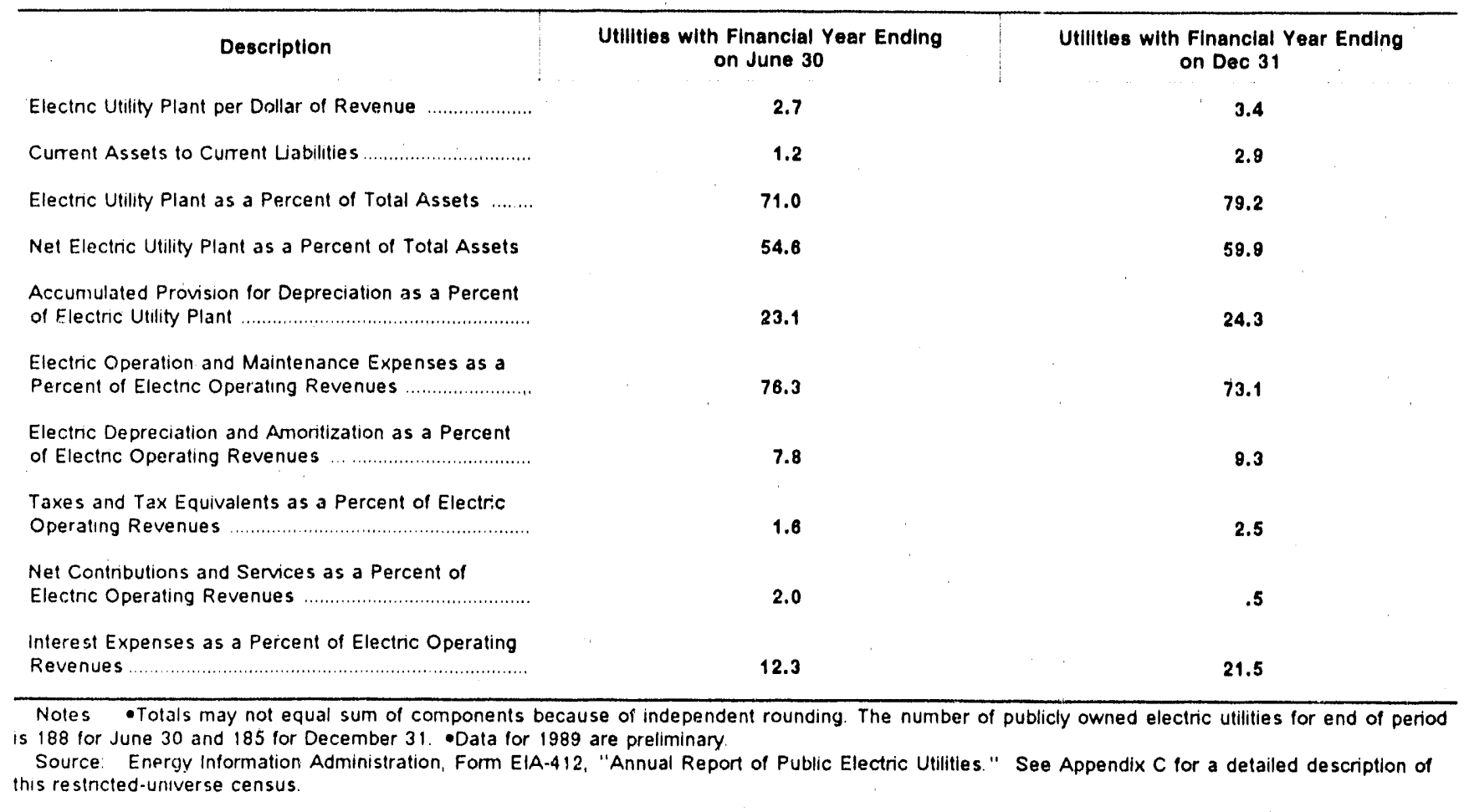

Table 39. Revenue and Expense Statistics for Selected Publicly Owned Electric Utilities, 1989 (Thousand Dollars)

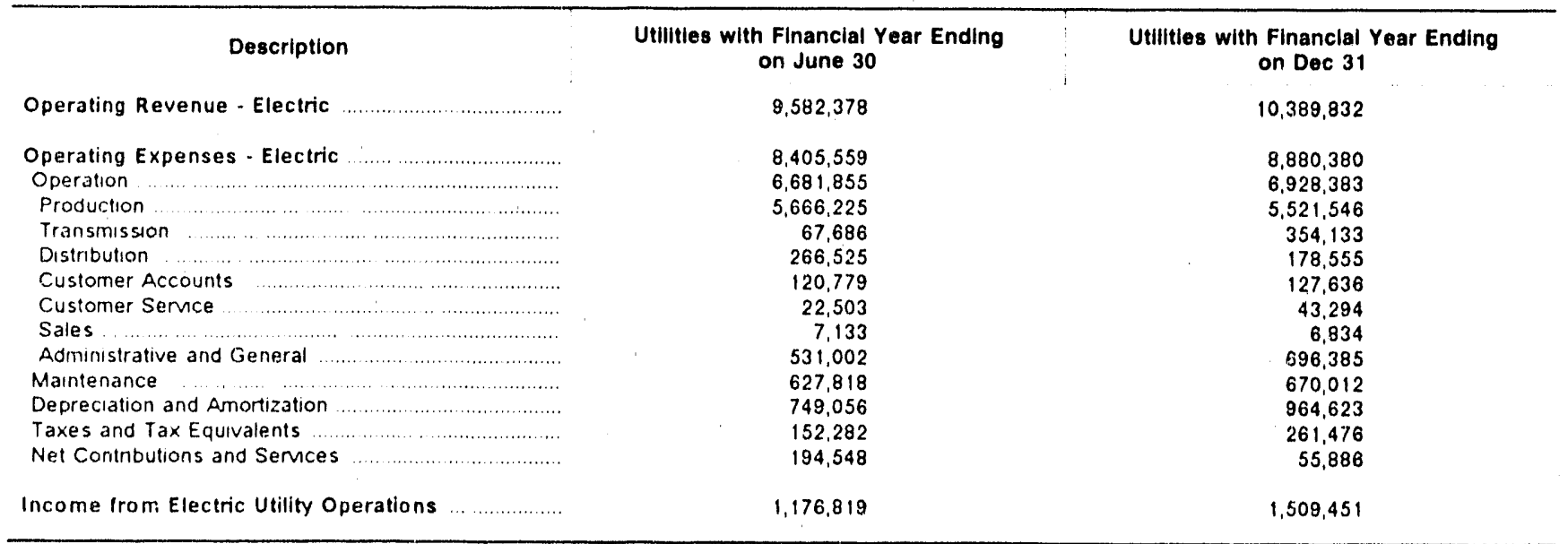

Notes Totals may not equal sum of components because of independent rounding. The number of publicly owned electric utilities for end of period is 188 for June 30 and 185 for December 31 . Data for 1989 are preliminary.

Source: Energy Information Administration, Form ElA.412. "Annual Report of Public Electric Utlities." See Appendix C for a detailed description of this resincted-universe census. 


\section{Environmental Statistics}

When fossil fuels are burned in the production of electricity, a variety of gases and particulates are formed. If these gases and particulates are not captured by some pollution control equipment, they are released into the atmosphere. This chapter provides a brief summary of these gaseous emissions and the methods employed to reduce or eliminate their release into the atmosphere.

\section{Background}

Among the gases emitted during the burning of fossil fuels are sulfur dioxide $\left(\mathrm{SO}_{2}\right)$, nitrogen oxides $\left(\mathrm{NO}_{x}\right)$, and carbon dioxide $\left(\mathrm{CO}_{2}\right)$. Coal-fired generating units produce more sulfur dioxide and nitrogen oxides than other fossil-fuel units for two reasons. First, because coal generally contains more sulfur than other fossil fuels, it creates more sulfur dioxide when burned. Second, more nitrogen oxide enissions are associated with coal-fired plants than with other fossil-fueled plants because mone coal-fired generating capacity is in use.

Sulfur is an element that is present in almost all coal, although some kinds of coal contain more sulfur than others depending on the geographic location of the coal mine and the type of coal being mined. Western coal has less sulfur than eastern coal. Over one-half of the coal mined in the West is subbituminous coal that is low in sulfur content (less than 0.5 percent) and contains approximately 8,000 Btu per pound. Eastern coal, or bituminous coal can exceed 8 percent sulfur and a heat content of over 12,000 Btu per pound. The average percent of sulfur contained in coal ranges from 0.3 percent in the West to approximately 2.5 percent in the East. During combustion, the sulfur combines with the oxygen in the air to form sulfur dioxide. As the sulfur dioxide mixes further with oxygen and trace substances in the air, a variety of sulfate compounds emerges. How these transformations take place, and in what proportions, is a subject of vigorous research. The behavior of sulfur emissions depends partly on the type of coal used and how it is burned. In addition, the presence of light, moisture, and other pollutants in the atmosphere may also be important in triggering the complex changes that sulfur emissions undergo. To a lesser degree, sulfur is also contained in petroleum and varies according to the type of petroleum (for example, light oil, heavy oil, etc.). Petroleum can range from almost no sulfur to about 5 percent sulfur. The average percent of sulfur contained in petroleum consumed by fossil-steam plants ranges from about 0.05 percent in western plants to about 2.75 percent for plants in the East. The amount of sulfur contained in gas is insignificant.

Nitrogen is a colorless, odorless gas that makes up about 78 percent of the atmosphere. Nitrogen combines with oxygen and water to form several nitrogen oxides. The most important is nitrogen dioxide, one of the compounds that gives photochemical smog its characteristic yellowish-brown color. Only about 10 percent of the nitrogen compounds in the air are the result of human activity. The rest are formed by natural processes, such as the decay of organic matter. However, since the human-made 10 percent is emitted mostly in industrial urban areas, concentration there can become high enough to cause concern.

Sulfur dioxide and nitrogen oxides are referred to as precursors to acid deposition, because, under the right set of conditions, they react with other chemicals in the atmosphere to form sulfuric acid and nitric acid, respectively. These two acids do not accumulate in the atmosphere, but are absorbed by rain droplets, thus cleansing the atmosphere and discharging acid onto the earth in the form of "acid rain." In addition, sulfuric acid may form microscopic droplets that can be deposited directly onto the ground. This form of deposition, as well as the direct capture of sulfur dioxide by vegetation, is referred to as dry deposition.

Carbon dioxide is a colorless, odorless, nontoxic gas formed by the combustion of carbon and carbon compounds found in coal, petroleum, and gas. Currently, the only way to limit the emission of carbon dioxide when burning fossil fuels is expensive. Efforts, however, are underway to determine what methods can be employed to reduce or eliminate the release of carbon dioxide from power plants. Tail gas cleanup (carbon dioxide scrubbing) is currently the only technological option. This option would require the adaptation by the electric utility industry of acid gas removal technologies used by the petroleum and petrochemical industries. Because of the potential expense involved, no emission standards or required reductions exist.

However, the Department of Energy is developing clean coal technologies (such as pressurized fluidized bed combustion) for new plants and repowering applications. Due to the increased conversion efficiencies of these technologies, carbon dioxide emissions are re- 
duced. For each 5-percentage-point improvement in conversion efficiency, carbon dioxide emissions are reduced by 10 to 15 percent. Carbon dioxide is normally removed from the atmosphere by green plants. The increased use of fossil fuels in recent years, as well as extensive deforestation, has caused a buildup of carbon dioxide in the atmosphere. This increase of carbon dioxide causes the atmosphere to absorb infrared radiation reflected from the earth that would otherwise have been dissipated into space. This phenomenon increases temperature and is called the "greenhouse" effect because it is similar to the trapping of the energy of the sun in a greenhouse. These increasing temperatures are of potential concern because they could cause dramatic climatic changes, shifts in agricultural zones, and partial melting of the polar ice caps resulting in flooding of coastal areas. However, significant uncertainties exist regarding global warming, and no conclusions can be drawn regarding future warming based on past temperature records.

\section{Emission Standards}

To respond to concerns about emissions of sulfur dioxide and nitrogen oxides as well as several other air pollutants, Congress passed the Clean Air Act (CAA) in 1963. It was not until 1970, however, that the Environmental Protection Agency was empowered to set enforceable air quality standards. In 1971, this Agency established New Source Performance Standards (NSPS) that required coal-fired utility boilers built after August 17, 1971, to emit no more than 1.2 pounds of sulfur dioxide per million Btu of heat input. Requirements for nitrogen oxides were more complex, with allowable limits ranging from 0.2 pounds per million Btu to 0.8 pounds per million Btu, depending on the type of fuel burned and the combustion device used.

In 1977, Congress amended the CA A to require States to set limits on existing sources in regions not attaining goals established in the Act. In 1979, the Environmental Protection Agency established the Revised New Source Performance Standards (RNSPS). The new standards retain the 1971 NSPS of 1.2 pounds of sulfur dioxide per million Btu of heat input, but require sulfur dioxide emissions from all new or modified (post 1978) boilers to be reduced by at least 90 percent unless 90-percent removal reduces emissions to less than 0.6 pounds per million Btu. If emissions fall below that level, reductions between 70 and 90 percent are permitted, depending on the sulfur content of the coal. RNSPS for nitrogen oxides are complex and, as with NSPS, set limits varying from 0.2 to 0.8 pounds per million Btu, depending on the type of fuel burned and combustion device used. RNSPS for nitrogen oxides differ from NSPS in the number of categories of combustion into which they are divided.

The Clean Air Act Amendments of 1990 was signed into law by President Bush on November 15, 1990. The goal of the bill is a 10-million-ton reduction in sulfur dioxide emissions and a 2-million-ton reduction in nitrogen oxide emissions from 1980 levels. The sulfur dioxide reduction is to be achieved in two phases. The first phase is a 5 -million-tion reduction to be achieved by 1995. The second phase is an additional 5-million-ton reduction to occur after 1999.

\section{Emission Reductions}

Sulfur Dioxide. One method available to reduce the sulfur dioxide emitted when burning coal, is to switch to a coal that has a lower sulfur content. Emissions of sulfur dioxide may also be reduced by using lesspolluting fuels, particularly gas. Another approach is to install equipment designed to remove sulfur dioxide from the gas (flue gas) released through the flues of the plant. Additional methods for reducing emissions of sulfur dioxide, which include converting boilers to the fluidized-bed combustion process and employing the technology of integrated-gasification combined cycle, are currently under study and not in extensive use.

Nitrogen Oxides. Formation of nitrogen oxides is less dependent on the type of fuel burned than on how the fuel is burned. Apart from the nitrogen content of the fuel, the extent of nitric-oxide formation depends primarily on the combustion temperature. Nitrogen-oxide emissions can be reduced by low-excess-air firing; lowcombustion temperatures; use of low-nitrogen fuels (such as natural gas and light distillate oil); staged combustion in which localized fuel-rich conditions are created where both thermal and fuel nitrogen oxides are minimized; and, use of low-nitrogen-oxide burners and fluidized-bed combustion.

\section{Environmental Equipment}

While not the only kind of environmental equipment installed at power plants, flue gas desulfurization units, particulate collectors, and cooling towers are the most significant. In a flue gas desulfurization unit (scrubber), the gases resulting from combustion are passed through tanks containing a material that captures and neutralizes the sulfur dioxide. Particulate matter is most frequently removed from the combustion gases by either filtering (a series of filter bags that trap the ash and dust much like a household vacuum cleaner) in a baghouse or with an electrostatic precipitator. In the latter, the particulates are given an electric charge and collected. Particulate collection is mainly centered on coal combustion because of the large percentage of ash that coal contains. Petroleum has very little ash and natural gas has practically none. A cooling tower is a structure for transferring heat in the water to the atmosphere. The most common type of cooling tower is the wet tower, also called the evaporative tower. In a wet tower, cooling is caused mainly by evaporation of the water and partly by direct-heat transer. 
For a fossil-fueled steam-electric generating unit, about two-thirds of the heat produced by burning the fuel is released to the environment, and only about one-third is used to produce electricity. Most waste heat (contained in the cooling water) is dissipated into a body of water, such as a river, lake, or bay. Cooling towers are installed where there is insufficient cooling water and where the waste heat discharged into the cooling water affects plants or marine life. ${ }^{2 k}$.

Environmental equipment can be a significant part of the cost of a power plant. This additional cost includes the initial capital cost of installation and the recurring operation and maintenance (O\&M) cost. Capital costs are given as a cost per kilowatt of installed nameplate capacity in dollars per megawatt.

\section{Data Sources}

Estimates are provided in the following tables on sulfur dioxide, nitrogen oxide, and carbon dioxide emissions from fossil-fueled steam-electric generating units. The methodology for computing emission estimates is described in Appendix C. Additional detailed information on emissions can be obtained in Chapter 4 and Appendix C of the Annual Outlook for U. S. Eiectric Power 1989." Also presented in the following tables are the number and capacity of fossil-fueled steamelectric generators with environmental equipment (scrubbers, particulate collectors, and cooling towers). Because power plants can have more than one type of environmental equipment, the generators at these plants can be included in more than one category. Also, not all plants have environmental equipment. Data regarding the quality of fossil fuels used to produce electricity, including heat, sulfur, and ash content, are also provided in the following tables. Lastly, average flue gas desulfurization costs (that is, operation and maintenance costs per kilowatthour of generation and installation costs per kilowatt of nameplate capacity) are presented.

These estimates were either derived or obtained directly from the Form EIA-767, "Steam-Electric Plant Operation and Design Report." This form is a restricted-universe census used to collect boilerspecific data from almost 900 power plants with a fossilfueled steam-electric nameplate capacity of 10 or more megawatts operated by over 300 electric utilities. The entire form, including data on environmental equipment, is filed by about 700 power plants with a nameplate capacity of 100 or more megawatts. Information on power plants with a nameplate capacity between 10 and 100 megawatts is submitted only for fuel consumption and flue gas desulfurization equipment.

\footnotetext{
${ }^{28}$ For a more detailed discussion of environmental equipment, see Energy Information Administration, Anmual Outlook for U.S. Electric Power DOE/EIA-(0474(85) (Washington, DC, August 1985).

${ }^{20}$ Energy lnformation Administration, Annual Oullook for U. S. Electric Power 1989 DOE/EIA-(0474(89) (Washingt(nn, DC, Junc 1989).
} 
Table 40. Emissions from Fossil-Fueled Steam-Electric Generating Units, 1985-1989 (Thousand Short Tons)

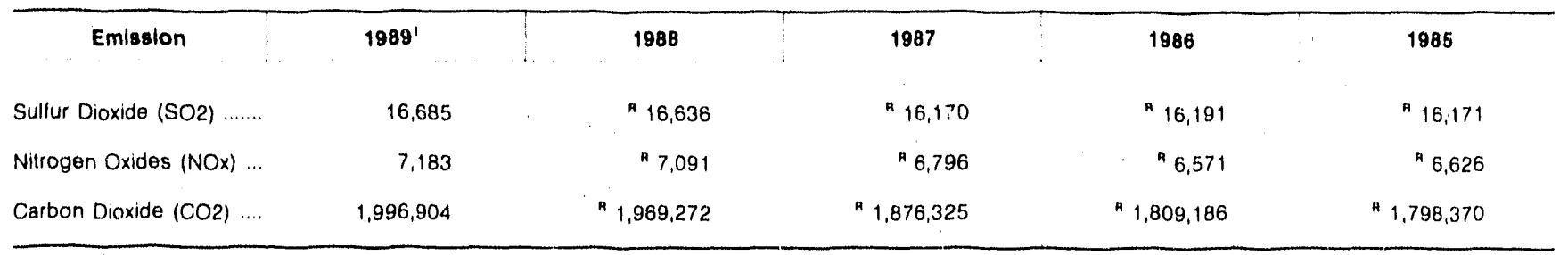

- Data for 1989 are preliminary.

$R=$ Revised data; data for 1985 through 1988 are revised. (See Appendix $C$ for an explanation).

Notes: -Data include petroleum coke. -These data are estimates derived from Form ElA.767, "Steam-Electric Plant Operation and Design Report." (See Appendix C for methodology).

Source: Energy Information Administration, Form EIA-767, "Steam.Electric Plant Operation and Design Report."

Table 41. Number and Capacity of Fossil-Fueled Steam-Electric Generators for Plants with Environmental Equipment, 1985-1989

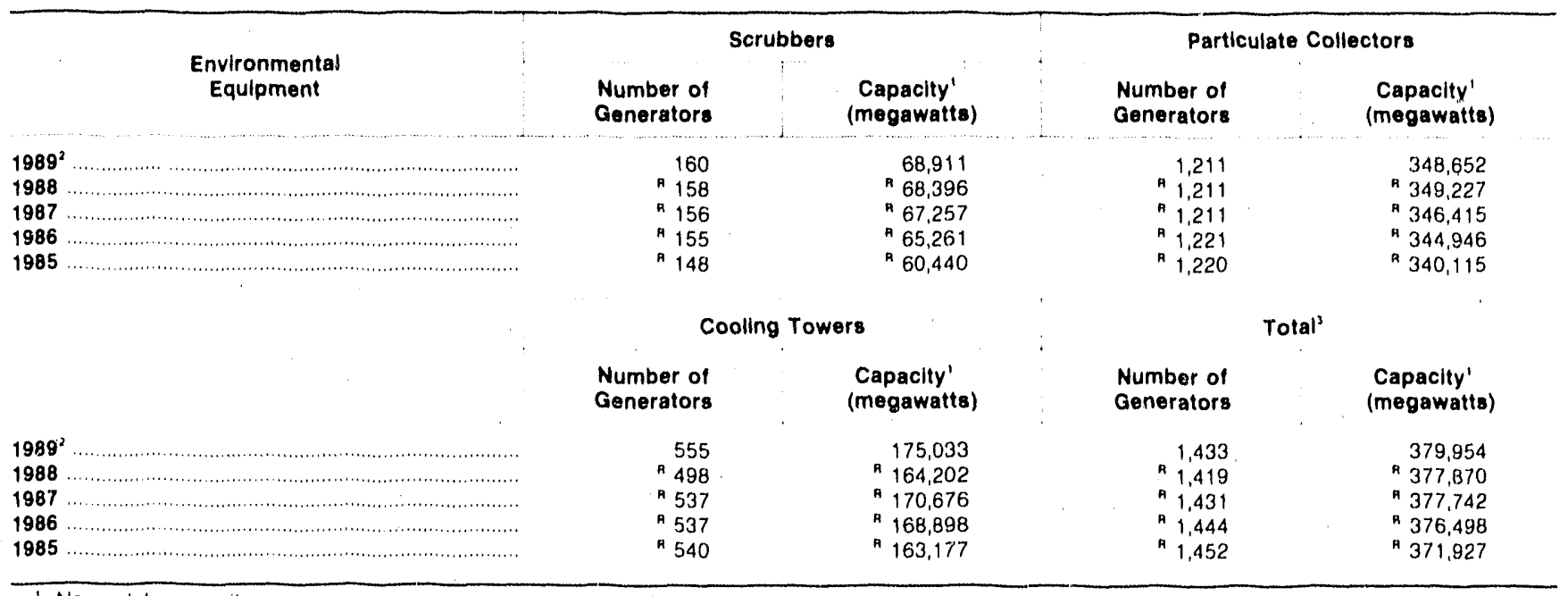

Nameplate capacity.

- Data for 1989 are preliminary.

- Components are not additive since some generators are included in more than one category and not all units have environmental equipment. R.: Revised data; data for 1985 through 1988 are revised. (See Appendix C for an explanation).

Notes: - These data are only for plants with a fossil-fueled stearn-electric capacity of 100 or more megawatts. - Data include petroleum coke. - These data are estimates derived from Form ElA.767, "Steam-Electric Plant Operation and Design Report.", (See Appendix C for methodology)

Source: Energy Intormation Administration, Form ElA-767. "Steam-Electric Plant Operation and Design Report." 
Table 42. Emissions from Fossil-Fueled Steam-Electric Generating Units by Census Division and State, 1989 and 1988 (Thousand Short Tons)

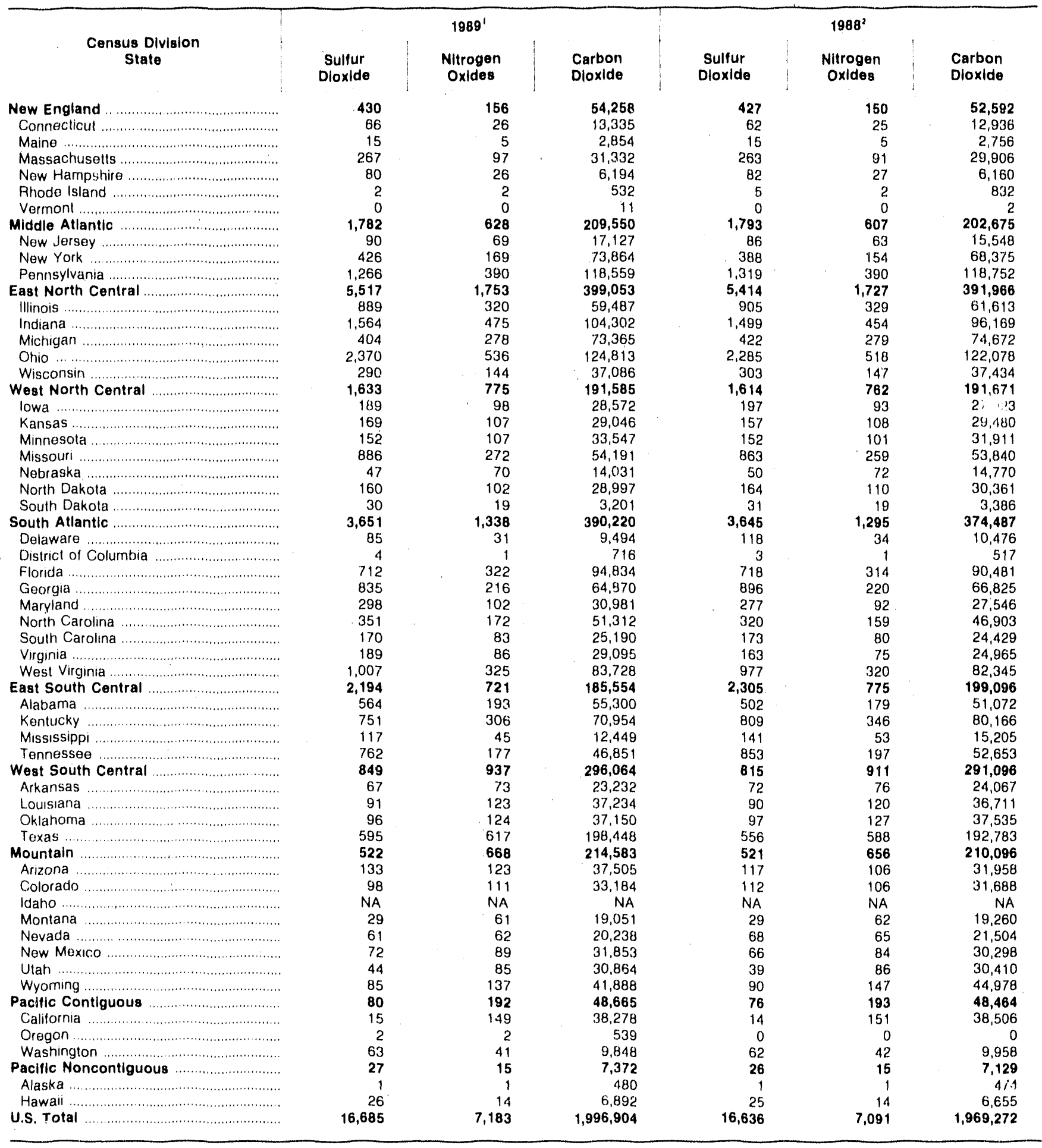

1 Data for 1989 are preliminary.

- Data for 1988 are revised; See Appendix C for an explanation. NA.. Nol applicable.

Notes: - Totals may not equal sum of components because of independent rounding. - Data include petroleum coke. - These data are estimates derived from Forrn ElA.767, "Steam-Electric Plant Operation and Design Report.", (See Appendix C tor methodology).

Source: Energy Information Administration, Form ElA.767. "Steam-Electric Plant Operation and Design Report." 
Table 43. Emissions from Fossil-Fueled Steam-Electric Generating Units by Fossil Fuel, Census Division; and State, 1989 (Thousand Short Tons)

\begin{tabular}{|c|c|c|c|c|c|c|c|c|c|c|}
\hline & & Coal & & & Petroleum & 4 & & & Gas & \\
\hline $\begin{array}{c}\text { Census Divislon } \\
\text { State }\end{array}$ & $\begin{array}{l}\text { Sulfur } \\
\text { Dloxide }\end{array}$ & $\begin{array}{c}\text { Nitrogen } \\
\text { Oxldes }\end{array}$ & $\begin{array}{l}\text { Carbon } \\
\text { Dloxide }\end{array}$ & $\begin{array}{l}\text { Sulfur } \\
\text { Dloxide }\end{array}$ & $\begin{array}{c}\text { Nitrogen } \\
\text { Oxides }\end{array}$ & $\begin{array}{l}\text { Carbon } \\
\text { Dloxide }\end{array}$ & $\begin{array}{l}\text { Sulfur } \\
\text { Dloxide }\end{array}$ & 1 & $\begin{array}{c}\text { Nitrogen } \\
\text { Oxides }\end{array}$ & $\begin{array}{l}\text { Carbon } \\
\text { Dioxide }\end{array}$ \\
\hline 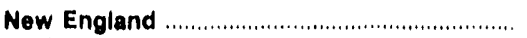 & 163 & 74 & 17,787 & $26 \%$ & 71 & 33,412 & & 0 & 11 & 3,059 \\
\hline 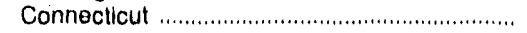 & 10 & 7 & 2,438 & 56 & 19 & 10,693 & & 0 & 0 & 204 \\
\hline 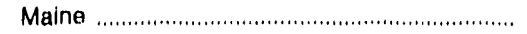 & 0 & 0 & 0 & 16 & 5 & 2,854 & & 0 & 0 & 0 \\
\hline 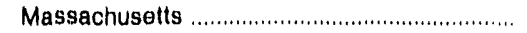 & 106 & 46 & 12,119 & 161 & 41 & 16,487 & & 0 & 10 & 2,726 \\
\hline 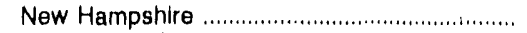 & 47 & 21 & 3,230 & 33 & 5 & 2,964 & & 0 & 0 & 0 \\
\hline 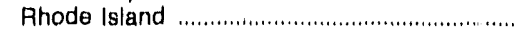 & 0 & 0 & 0 & 2 & 1 & 407 & & 0 & 1 & 125 \\
\hline 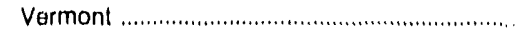 & 0 & 0 & 0 & 0 & 0 & 7 & & 0 & 0 & 4 \\
\hline 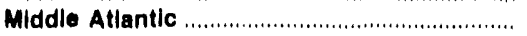 & 1,597 & 497 & 148,107 & 185 & 91 & 48,417 & & 0 & 40 & 13,026 \\
\hline New Jersey & 76 & 47 & 9,379 & 14 & 12 & 5,265 & & 0 & 10 & 2,483 \\
\hline 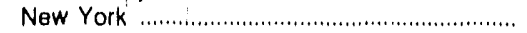 & 267 & 71 & 26,949 & 159 & 68 & 36,466 & & 0 & 30 & 10,449 \\
\hline 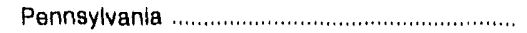 & 1,254 & 379 & $111.77 \dot{y}$ & 12 & 11 & 6,686 & & 0 & 0 & 94 \\
\hline 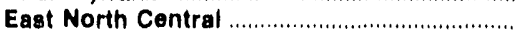 & 5,505 & 1,725 & 391,266 & 12 & 7 & 3,342 & & 0 & 21 & 4,445 \\
\hline 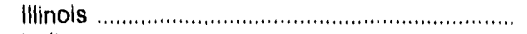 & 886 & 317 & 58,007 & 3 & 2 & 1,085 & & 0 & 1 & 395 \\
\hline 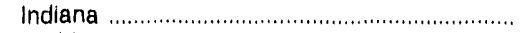 & 1,564 & 456 & 100,581 & 0 & 1 & 185 & & 0 & 18 & 3,536 \\
\hline Michigan & 397 & 273 & 71,444 & 7 & 3 & 1,541 & & 0 & 2 & 380 \\
\hline Ohio & 2,368 & 535 & 124,287 & 2 & 1 & 489 & & 0 & 0 & 37 \\
\hline Wisconsin & 290 & 144 & 36,947 & 0 & 0 & 42 & & 0 & 0 & 97 \\
\hline West North Central.. & 1,632 & 770 & 189,715 & 1 & 0 & 308 & & 0 & $\boldsymbol{b}$ & 1,562 \\
\hline lowa & 189 & 97 & 28,399 & 0 & 0 & 31 & & 0 & 1 & 142 \\
\hline 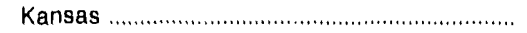 & 169 & 104 & 27,982 & 0 & 0 & 68 & & 0 & 3 & 996 \\
\hline 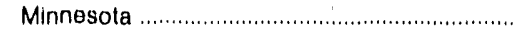 & 152 & 106 & 33,270 & 0 & 0 & 32 & & 0 & 1 & 245 \\
\hline 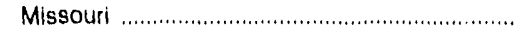 & 885 & 272 & 54,060 & 1 & 0 & 92 & & 0 & 0 & 39 \\
\hline Nebraska & 47 & 70 & 13,857 & 0 & 0 & 42 & & 0 & 0 & 132 \\
\hline 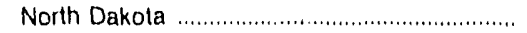 & 160 & 102 & 28,962 & 0 & 0 & 35 & & 0 & 0 & 0 \\
\hline South Dakota & 30 & 19 & 3,185 & 0 & 0 & 8 & & 0 & 0 & 8 \\
\hline 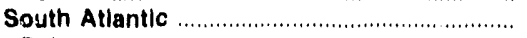 & 3,401 & 1,214 & 342,290 & 250 & 83 & 36,330 & & 0 & 41 & 11,600 \\
\hline Delaware & 70 & 20 & 6,548 & 15 & 8 & 2,266 & & 0 & 3 & 680 \\
\hline 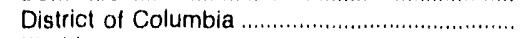 & 0 & 0 & 0 & 4 & 1 & 716 & & 0 & 0 & 0 \\
\hline 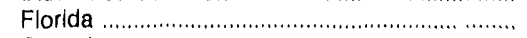 & 544 & 235 & 62,127 & 168 & 52 & 23,243 & & 0 & 35 & 9,464 \\
\hline 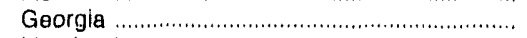 & 835 & 216 & 64,751 & 0 & 0 & 88 & & 0 & 0 & 31 \\
\hline Maryland & 252 & 84 & 23,845 & 46 & 15 & 6,051 & & 0 & 3 & 1,085 \\
\hline North Carolina & 351 & 172 & 51,156 & 0 & 0 & 156 & & 0 & 0 & 0 \\
\hline South Carolina & 170 & 83 & 25,013 & 0 & 0 & 63 & & 0 & 0 & 114 \\
\hline 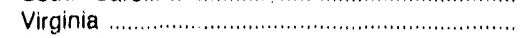 & 172 & 80 & 25,340 & 17 & 6 & 3,536 & & 0 & 0 & 219 \\
\hline 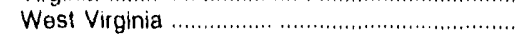 & 1,007 & 324 & 83,510 & 0 & 1 & 211 & & 0 & 0 & 7 \\
\hline 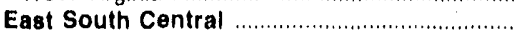 & 2,182 & 709 & 182,069 & 12 & 3 & 1,087 & & 0 & 9 & 2,398 \\
\hline 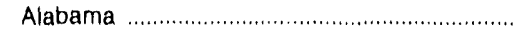 & 564 & 193 & 55,106 & 0 & 0 & 88 & & 0 & 0 & 106 \\
\hline 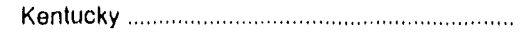 & 751 & 306 & 70,809 & 0 & 0 & 125 & & 0 & 0 & 20 \\
\hline 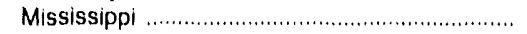 & 105 & 33 & 9,405 & 12 & 3 & 772 & & 0 & 9 & 2,272 \\
\hline Tennesse日 & 762 & 177 & 46,749 & 0 & 0 & 102 & & 0 & 0 & 0 \\
\hline 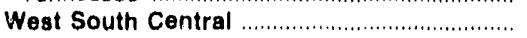 & 840 & 615 & 206,678 & 9 & 8 & 2,556 & & 0 & 314 & 86,830 \\
\hline 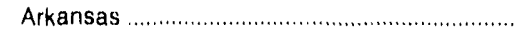 & 66 & $6 B$ & 21,289 & 1 & 0 & 135 & & 0 & 5 & 1,808 \\
\hline 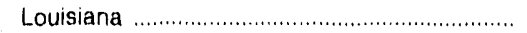 & 90 & 71 & 22,247 & 1 & 1 & 276 & & 0 & 51 & 14.711 \\
\hline Oklahoma & 96 & 85 & 27,683 & 0 & 0 & 27 & & 0 & 39 & 9,440 \\
\hline 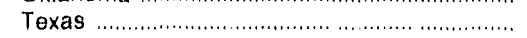 & 588 & 391 & 135,459 & 7 & 7 & 2,118 & & 0 & 219 & 60,871 \\
\hline 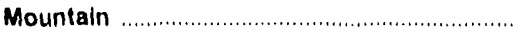 & 520 & 650 & 208,691 & 2 & 1 & 696 & & 0 & 17 & 5,196 \\
\hline 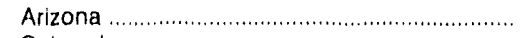 & 133 & 117 & 35,582 & 0 & 0 & 154 & & 0 & 6 & 1,769 \\
\hline 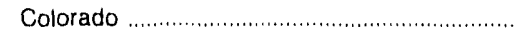 & 98 & 109 & 32,718 & 0 & 0 & 26 & & 0 & 2 & 440 \\
\hline 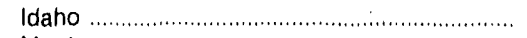 & 0 & 0 & 0 & 0 & 0 & 0 & & 0 & 0 & 0 \\
\hline Montana & 29 & 61 & $19,00 ?$ & 0 & 0 & 23 & & 0 & 0 & 26 \\
\hline 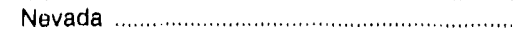 & 59 & 56 & 18,590 & 2 & 1 & 348 & & 0 & 5 & 1,300 \\
\hline 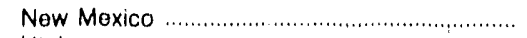 & 72. & 85 & 30,158 & 0 & 0 & 43 & & 0 & 4 & 1,652 \\
\hline 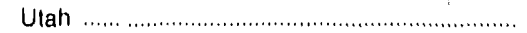 & 44 & 85 & 30,820 & 0 & 0 & 40 & & 0 & 0 & 4 \\
\hline 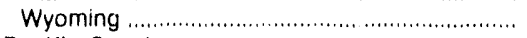 & $8 s^{5}$ & 137 & 41,821 & 0 & 0 & 62 & & 0 & 0 & 5 \\
\hline Pacific Contiguous & 64 & 43 & 10,291 & 16 & 28 & 8,518 & & 0 & 121 & 29,856 \\
\hline California & 0 & 0 & 0 & 15 & 28 & 8,422 & & 0 & 121 & 29,856 \\
\hline Oregon & 2 & 2 & 534 & 0 & 0 & 5 & & 0 & 0 & 0 \\
\hline 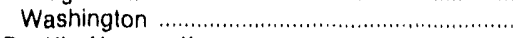 & 62 & 41 & 9,757 & 1 & 0 & 91 & & 0 & 0 & 0 \\
\hline 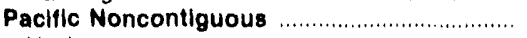 & 1 & 1 & 479 & 26 & 14 & 6,893 & & 0 & 0 & 0 \\
\hline Alaska .............................. & 1 & 1 & 479 & 0 & 0 & 1 & & 0 & 0 & 0 \\
\hline Hawaii & 0 & 0 & 0 & 26 & 14 & 6,892 & & 0 & 0 & 0 \\
\hline …, & 15,905 & 6,298 & $1,697,373$ & 780 & 306 & 141,559 & & 0 & 579 & 157,972 \\
\hline
\end{tabular}

Notes: - Totals may not equal sum of components because of independent rounding. •Data for 1989 are preliminary. $\bullet$ Data include petroleum coke. - These data are estimates derived from Form EIA-767. "Steam-Electric Plant Operation and Design Report.", (Sese Appendix C for methodology) Source: Energy Information Administration, Form ElA-767, "Steam-Electric Plant Operation and Design Report." 
Table 44. Number and Capacity of Coal-Fired Steam-Electric Generators for Plants with Environmental Equipment by Census Division and State, 1989

\begin{tabular}{|c|c|c|c|c|c|c|c|c|}
\hline Census Division ' & $\begin{array}{c}\text { Gene } \\
\text { Un }\end{array}$ & $\begin{array}{l}\text { rating } \\
\text { ts }^{\prime}\end{array}$ & Soru & bbers & $\begin{array}{l}\text { Partl } \\
\text { Colle }\end{array}$ & $\begin{array}{l}\text { oulate } \\
\text { ctors }\end{array}$ & Coollng & Towers \\
\hline State & $\begin{array}{l}\text { Number of } \\
\text { Generators }\end{array}$ & $\begin{array}{c}\text { Capaoity } \\
\text { (megawatts) }\end{array}$ & $\begin{array}{l}\text { Number of } \\
\text { Generators }\end{array}$ & $\begin{array}{c}\text { Capaclty }^{2} \\
\text { (megawatts) }\end{array}$ & $\begin{array}{l}\text { Number of } \\
\text { Generators }\end{array}$ & $\begin{array}{c}\text { Capaolty } \\
\text { (megawatts) }\end{array}$ & $\begin{array}{l}\text { Number of } \\
\text { Generators }\end{array}$ & $\begin{array}{c}\text { Capaclty } \\
\text { (megawatts) }\end{array}$ \\
\hline 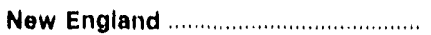 & 12 & 2,623 & 0 & 0 & 12 & 2,623 & 0 & 0 \\
\hline 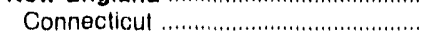 & 1 & 400 & 0 & 0 & 1 & 400 & 0 & 0 \\
\hline 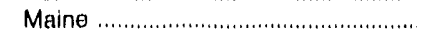 & 0 & 0 & 0 & 0 & 0 & 0 & 0 & 0 \\
\hline 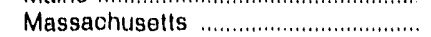 & 9 & 1,764 & 0 & 0 & 9 & 1,764 & 0 & 0 \\
\hline New Hampshire .................................... & 2 & 450 & 0 & 0 & 2 & 459 & 0 & 0 \\
\hline 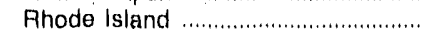 & $\overrightarrow{0}$ & 0 & 0 & 0 & 0 & 0 & 0 & 0 \\
\hline 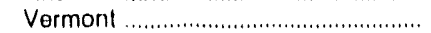 & 0 & 0 & 0 & 0 & 0 & 0 & 0 & 0 \\
\hline Middle Atlantlc & 94 & 24,669 & 16 & 5,512 & 94 & 24,669 & 20 & 12,657 \\
\hline 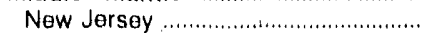 & 8 & 1,732 & 0 & 0 & 8 & 1,732 & 0 & 0 \\
\hline Now York & 25 & 3,743 & 1 & 655 & 25 & 3,743 & 0 & 0 \\
\hline 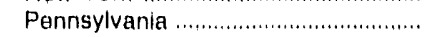 & 61 & 19,194 & 15 & 4,857 & 61 & 19,194 & 20 & 12,657 \\
\hline East North Central ............................. & 306 & 81,341 & 17 & 7,216 & 309 & 81,486 & 46 & 20,979 \\
\hline 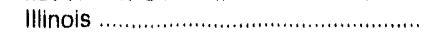 & 55 & 17,123 & 4 & 1,439 & 55 & 17,123 & 3 & 671 \\
\hline 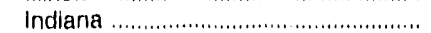 & 69 & 21,703 & 11 & 4,890 & 72 & 21,847 & 20 & 9,207 \\
\hline Michigan & 55 & 12,659 & 0 & 0 & 55 & 12,659 & 7 & 536 \\
\hline Ohio & 87 & 22,732 & 2 & 888 & 87 & 22,732 & 11 & 7,986 \\
\hline Wisconsin & 40 & 7,126 & 0 & 0 & 40 & 7,126 & 5 & 2,579 \\
\hline West North Central & 140 & 35,924 & 27 & 11,539 & 140 & 35,924 & 49 & 12,914 \\
\hline lowa & 31 & 5,857 & 1 & 160 & 31 & 5,857 & 9 & 1,912 \\
\hline Kansas & 19 & 5,712 & 8 & 3,995 & 19 & 5,712 & 10 & 3,435 \\
\hline 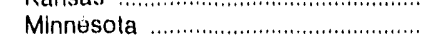 & 25 & 5,350 & 8 & 3,305 & 25 & 5,350 & 9 & 3,787 \\
\hline 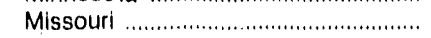 & 38 & 11,517 & 3 & 1,099 & 38 & 11,517 & 12 & 1,019 \\
\hline 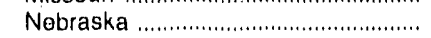 & 14 & 3,038 & 0 & 0 & 14 & 3,038 & 4 & 429 \\
\hline 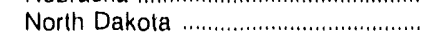 & 12 & 3,994 & 7 & 2,981 & 12 & 3,994 & 5 & 2,332 \\
\hline South Dakota & 1 & 456 & 0 & 0 & 1 & 456 & 0 & 0 \\
\hline 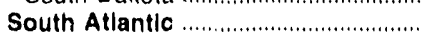 & 206 & 65,840 & 13 & 6,752 & 204 & 65,778 & 66 & 34,796 \\
\hline Delaware & 6 & 1,034 & 0 & 0 & 6 & 1,034 & 1 & 442 \\
\hline District of Columbia & 0 & 0 & 0 & 0 & 0 & 0 & 0 & 0 \\
\hline 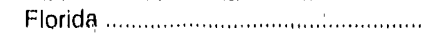 & 27 & 10,658 & 7 & 3,883 & 27 & 10,658 & 13 & 6,264 \\
\hline Georgia & 38 & 12,957 & 0 & 0 & 38 & 12,957 & 12 & 8,862 \\
\hline Maryland & 14 & 4,257 & 0 & 0 & 14 & 4,257 & 1 & 685 \\
\hline North Carolina & 40 & 12,101 & 0 & 0 & 38 & 12,039 & 8 & 3,188 \\
\hline South Carolina & 24 & 5,324 & 4 & 1,501 & 2.4 & 5,324 & 14 & 4,139 \\
\hline 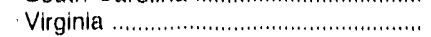 & 24 & 4,549 & 0 & 0 & 24 & 4,549 & 3 & 713 \\
\hline West Virginia & 33 & 14,958 & 2 & 1,368 & 33 & 14,958 & 14. & 10,506 \\
\hline 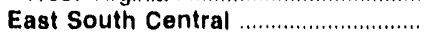 & 135 & 39,326 & 22 & 7,873 & 135 & 39,326 & 33 & 15,408 \\
\hline Alabama & 39 & 11,943 & 4 & 1,591 & 39 & 11,943 & 6 & 3,535 \\
\hline 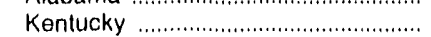 & 53 & 15,212 & 16 & 5,882 & 53 & 15,212 & 23 & 10,724 \\
\hline Mississippi .......................................... & 6 & 2,150 & 2 & 400 & 6 & 2,150 & 4 & 1,150 \\
\hline 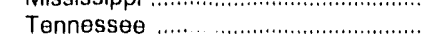 & 37 & 10,020 & 0 & 0 & 37 & 10,020 & 0 & 0 \\
\hline 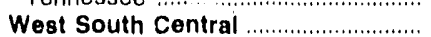 & 54 & 32,458 & 15 & 9,891 & 54 & $32,4 ! i 8$ & 30 & 17,746 \\
\hline Arkansas & 5 & 3,958 & 0 & 0 & 5 & 3,558 & 4 & 3,400 \\
\hline 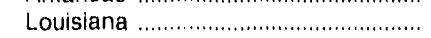 & 6 & 3,572 & 1 & 721 & 6 & 3,572 & 4 & 2,454 \\
\hline 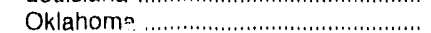 & 10 & 5,210 & 1 & 520 & 10 & 5,210 & 8 & 4,072 \\
\hline Texas & 33 & 19,719 & 13 & 8,650 & 33 & 19,710 & 14 & 7,820 \\
\hline Mountain & 89 & 30,383 & 47 & 20,063 & 85 & 28,604 & 78 & 26,736 \\
\hline Arizona & 13 & 5,297 & 8 & 2,425 & 13 & 5,297 & 11 & 4,894 \\
\hline Colorado & 26 & 4,946 & 4 & 1,594 & 26 & 4,946 & 24 & 4,524 \\
\hline 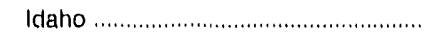 & NA & NA & NA & NA & NA & NA & NA & NA \\
\hline Montana & 5 & 2,464 & 4 & 2,273 & 5 & 2,464 & 4 & 2,273 \\
\hline Nevada & 8 & 2,769 & 5 & 879 & 8 & 2,769 & 8 & 2,769 \\
\hline 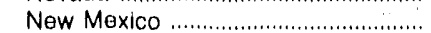 & 10 & 4,282 & 10 & 4,282 & 6 & 2,503 & 5 & 2,012 \\
\hline Utah & 12 & 4,787 & 7 & 3,969 & 12 & 4,787 & 12 & 4,787 \\
\hline 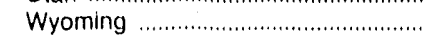 & 15 & 5,838 & 9 & 4,641 & 15 & 5,838 & 14 & 5,476 \\
\hline Pacific Contiguous & 3 & 2,020 & 0 & 0 & 3 & 2,020 & 2 & 1,460 \\
\hline 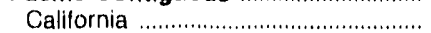 & 0 & 0 & 0 & 0 & 0 & 0 & $\overline{0}$ & 0 \\
\hline Oregon & 1 & 561 & 0 & 0 & 1 & 561 & 0 & 0 \\
\hline Washington & 2 & 1,460 & 0 & 0 & 2 & 1,460 & 2 & 1,460 \\
\hline Pacific Nonoontiguous & 0 & 0 & 0 & 0 & 0 & 0 & 0 & 0 \\
\hline 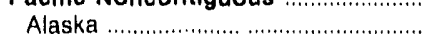 & 0 & 0 & 0 & 0 & 0 & 0 & 0 & $\dot{0}$ \\
\hline 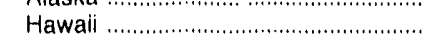 & 0 & 0 & 0 & 0 & 0 & 0 & 0 & 0 \\
\hline U.S. Total & 1,039 & 314,585 & 157 & 68,846 & 1,036 & 312,887 & 324 & 142,696 \\
\hline
\end{tabular}

1 Components are not additive since some generators are included in more than one category and not all units have environmental equipment.

- Nameplate capacity.

NA Not applicable.

Notes: - Totals may not equal sum of components because of independent rounding. - These data are only for plants with a fossil-fueled steamelectric capacity of 100 or more megawatts. - Data for 1989 are prelliminary. Data include petroleum coke.

Source: Energy Information Administration, Form EIA-767. "Steam-Electric Plant Operation and Design Report." 
Table 45. Number and Capacity of Petroleum- and Gas-Fired Steam-Electric Generators for Plants with Environmental Equipment by Census Division and State, 1989

\begin{tabular}{|c|c|c|c|c|c|c|c|c|}
\hline \multirow{2}{*}{$\begin{array}{c}\text { Census Division } \\
\text { State }\end{array}$} & \multicolumn{2}{|c|}{$\begin{array}{l}\text { Generating } \\
\text { Unitg' }\end{array}$} & \multicolumn{2}{|c|}{ Scrubbers } & \multicolumn{2}{|c|}{$\begin{array}{l}\text { Particulate } \\
\text { Collectors }\end{array}$} & \multicolumn{2}{|c|}{ Cooling Towers } \\
\hline & $\begin{array}{l}\text { Number of } \\
\text { Generators }\end{array}$ & $\begin{array}{c}\text { Capacity } \\
\text { (megawatt8) }\end{array}$ & $\begin{array}{l}\text { Number of } \\
\text { Generators }\end{array}$ & $\begin{array}{c}\text { Capaclty } \\
\text { (megawatts) }\end{array}$ & $\begin{array}{l}\text { Number of } \\
\text { Generators }\end{array}$ & $\begin{array}{c}\text { Capaoity } \\
\text { (megawatts) }\end{array}$ & $\begin{array}{l}\text { Nuinber of } \\
\text { Generators }\end{array}$ & $\begin{array}{c}\text { Capaoity } \\
\text { (megawatts) }\end{array}$ \\
\hline New England & 44 & 7,459 & 0 & 0 & 43 & 7,044 & 1 & 415 \\
\hline 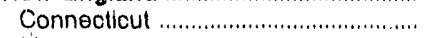 & 17 & 2,803 & 0 & 0 & 16 & 2,389 & 1 & 415 \\
\hline Maine & 9 & 993 & 0 & 0 & 9 & 993 & 0 & 0 \\
\hline 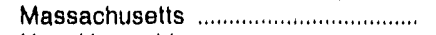 & 8 & 2,850 & 0 & 0 & 8 & 2,850 & 0 & 0 \\
\hline New Hampshire ..................................... & 4 & 564 & 0 & 0 & 4 & 564 & 0 & 0 \\
\hline 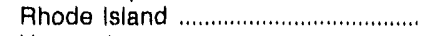 & 6 & 249 & 0 & 0 & 6 & 249 & 0 & 0 \\
\hline 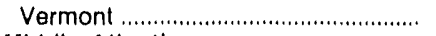 & 0 & 0 & 0 & 0 & 0 & 0 & 0 & 0 \\
\hline 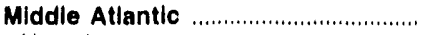 & 57 & 13,552 & 0 & 0 & 54 & 11,716 & 4 & 2,012 \\
\hline New Jersey & 14 & 2,130 & 0 & 0 & 13 & 1.995 & 2 & 311 \\
\hline 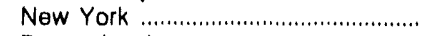 & 31 & 7,841 & 0 & 0 & 31 & 7,841 & 0 & 0 \\
\hline 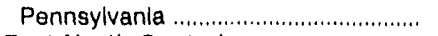 & 12 & 3,581 & 0 & 0 & 10 & 1,880 & 2 & 1.701 \\
\hline East North Central ................................. & 15 & 2,315 & 0 & 0 & 9 & 730 & 3 & 1,441 \\
\hline 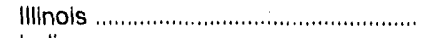 & 1 & 210 & 0 & 0 & 0 & 0 & 1 & 210 \\
\hline 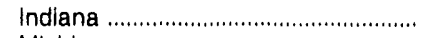 & 5 & 284 & 0 & 0 & 2 & 140 & 0 & 0 \\
\hline 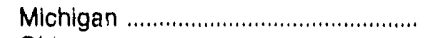 & 6 & 1,743 & 0 & 0 & 4 & 512 & 2 & 1,231 \\
\hline 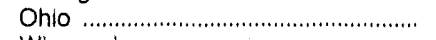 & 0 & 0 & 0 & 0 & 0 & 0 & 0 & 0 \\
\hline 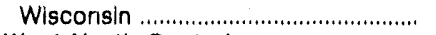 & 3 & 78 & 0 & 0 & 3 & 78 & 0 & 0 \\
\hline West North Central .............................. & 20 & 1,690 & 0 & 0 & 6 & 204 & 17 & 1,547 \\
\hline lowa & 1 & 19 & 0 & 0 & 1 & 19 & 0 & 0 \\
\hline 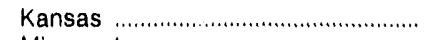 & 15 & 1,536 & 0 & 0 & 1 & 50 & 14 & 1,486 \\
\hline 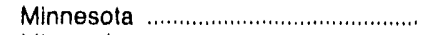 & 1 & 75 & 0 & 0 & 1 & 75 & 0 & 0 \\
\hline Missouri ............................................. & 3 & 61 & 0 & 0 & 3 & 61 & 3 & 61 \\
\hline 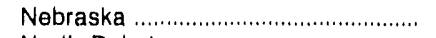 & 0 & 0 & 0 & 0 & 0 & 0 & 0 & 0 \\
\hline 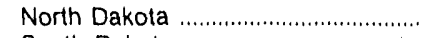 & 0 & 0 & 0 & 0 & 0 & 0 & 0 & 0 \\
\hline 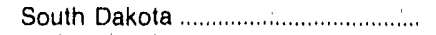 & 0 & 0 & 0 & 0 & 0 & 0 & 0 & 0 \\
\hline South Atlantlc & 67 & 16,745 & 3 & 65 & 49 & 12,843 & 20 & 4,946 \\
\hline 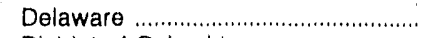 & 7 & 662 & 3 & 65 & 4 & 511 & 3 & 151 \\
\hline District of Columbia ............................ & 2 & 580 & 0 & 0 & 0 & 0 & 2 & 580 \\
\hline 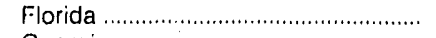 & 34 & 10,552 & 0 & 0 & 23 & 8.700 & 11 & 1,853 \\
\hline 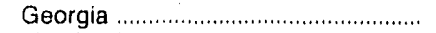 & 0 & 0 & 0 & 0 & 0 & 0 & 0 & 0 \\
\hline 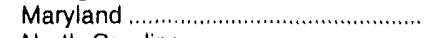 & 13 & 2,594 & 0 & 0 & 11 & 1,276 & 3 & 1,480 \\
\hline 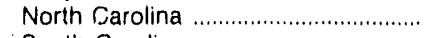 & 7 & 455 & 0 & 0 & 7 & 455 & 0 & 0 \\
\hline 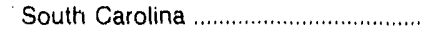 & 0 & 0 & 0 & 0 & 0 & 0 & 0 & 0 \\
\hline 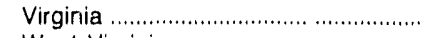 & 4 & 1,902 & 0 & 0 & 4 & 1,902 & 1 & 882 \\
\hline 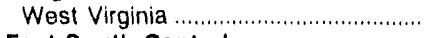 & 0 & 0 & 0 & 0 & 0 & 0 & 0 & 0 \\
\hline 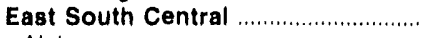 & 10 & 846 & 0 & 0 & 1 & 147 & 9 & 699 \\
\hline 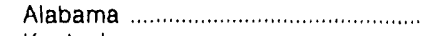 & 0 & 0 & 0 & 0 & 0 & 0 & 0 & 0 \\
\hline 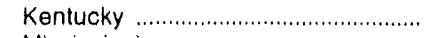 & 1 & 147 & 0 & 0 & 1 & 147 & 0 & 0 \\
\hline Mississippi …................................... & 9 & 690 & 0 & 0 & 0 & 0 & 9 & 699 \\
\hline Tennessee & 0 & 0 & 0 & 0 & 0 & 0 & 0 & 0 \\
\hline 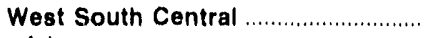 & 105 & 15,938 & 0 & 0 & 5 & 2,452 & 103 & 14,780 \\
\hline 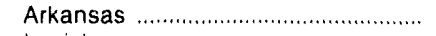 & 7 & 580 & 0 & 0 & 0 & 0 & 7 & 580 \\
\hline 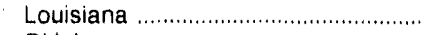 & 18 & 2,728 & 0 & 0 & 2. & 1,184 & 17 & 2,136 \\
\hline 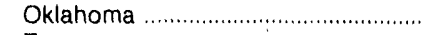 & 19 & 4,350 & 0 & 0 & 1 & 567 & 18 & 3,783 \\
\hline 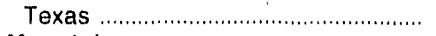 & 61 & 8,281 & 0 & 0 & 2 & 701 & 61 & 8,281 \\
\hline 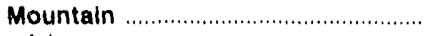 & 43 & 3,120 & 0 & 0 & 2 & 101 & 43 & 3,120 \\
\hline Arizona & 20 & 1,597 & 0 & 0 & 0 & 0 & 20 & 1,597 \\
\hline 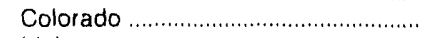 & 3 & 111 & 0 & 0 & 2 & 101 & 3 & 111 \\
\hline 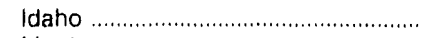 & NA & NA & NA & NA & NA & NA & NA & NA \\
\hline 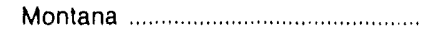 & 0 & 0 & 0 & 0 & 0 & 0 & 0 & 0 \\
\hline 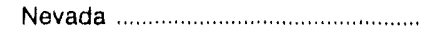 & 6 & 433 & 0 & 0 & 0 & 0 & 6 & 433 \\
\hline 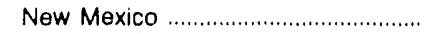 & 13 & 910 & 0 & 0 & 0 & 0 & 13 & 910 \\
\hline 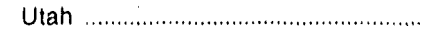 & 1 & 69 & 0 & 0 & 0 & 0 & 1 & 69 \\
\hline 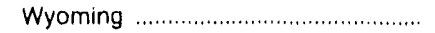 & 0 & 0 & 0 & 0 & 0 & 0 & 0 & 0 \\
\hline 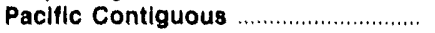 & 33 & 3,705 & 0 & 0 & 6 & 527 & 31 & 3,379 \\
\hline California & 33 & 3,705 & 0 & 0 & 6 & 527 & 31 & 3,379 \\
\hline 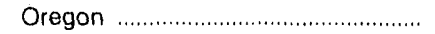 & 0 & 0 & 0 & 0 & 0 & 0 & 0 & 0 \\
\hline 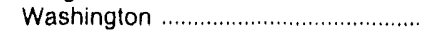 & 0 & 0 & 0 & 0 & 0 & 0 & 0 & 0 \\
\hline Pacific Noncontiguous ...................... & 0 & 0 & 0 & 0 & 0 & 0 & 0 & 0 \\
\hline Alaska & 0 & 0 & 0 & 0 & 0 & 0 & 0 & 0 \\
\hline 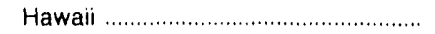 & 0 & 0 & 0 & 0 & 0 & 0 & 0 & 0 \\
\hline 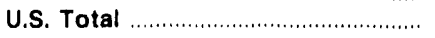 & 394 & 65,370 & 3 & 65 & 175 & 35,764 & 231 & 32,337 \\
\hline
\end{tabular}

1 Components are not additive since some generators are included in more than one category and not all units have environmental equipment.

2 Nameplate capacity.

NA $=$ Not applicable

Notes: -Totals may not equal sum of components because of independent rounding. -These data are oniy for plants with a fossil.fueled steamelectric capacity of 100 or more megawatts. Data for 1989 are preliminary. Data include petroleum coke.

Source: Energy Information Administration, Form ElA-i67, "Steam-Electric Plant Operation and Design Report." 
Table 46. Average Quality of Fossil Fuels Burned by Census Jivision and State, 1989 and 1988

\begin{tabular}{|c|c|c|c|c|c|c|c|c|c|c|c|c|}
\hline \multirow[b]{3}{*}{$\begin{array}{l}\text { Census Division } \\
\text { Siate }\end{array}$} & \multicolumn{6}{|c|}{ Coal } & \multicolumn{4}{|c|}{ Petroleum } & \multicolumn{2}{|c|}{ Gas } \\
\hline & \multicolumn{3}{|c|}{$1989^{\prime}$} & \multicolumn{3}{|c|}{$1988^{2}$} & \multicolumn{2}{|c|}{$1989^{\prime}$} & \multicolumn{2}{|l|}{$1988^{2}$} & \multirow[b]{2}{*}{$\begin{array}{l}1989^{\prime} \\
\text { Average } \\
\text { Btu per } \\
\text { Cublc } \\
\text { Foot }\end{array}$} & \multirow[b]{2}{*}{$\begin{array}{l}1988 \\
\text { Average } \\
\text { Btu per } \\
\text { Cublc } \\
\text { Foot }\end{array}$} \\
\hline & $\begin{array}{l}\text { Average } \\
\text { Btu } \\
\text { per } \\
\text { Pound }\end{array}$ & $\begin{array}{l}\text { Suifur } \\
\text { Percent } \\
\text { by } \\
\text { Welght }\end{array}$ & $\begin{array}{c}\text { Ash } \\
\text { Percent } \\
\text { by } \\
\text { Welght }\end{array}$ & $\begin{array}{c}\text { Average } \\
\text { Btu } \\
\text { per } \\
\text { Pound }\end{array}$ & $\begin{array}{c}\text { Sulfur } \\
\text { Percent } \\
\text { by } \\
\text { Weight }\end{array}$ & $\begin{array}{c}\text { Ash } \\
\text { Percent } \\
\text { by } \\
\text { Weight }\end{array}$ & $\begin{array}{l}\text { Average } \\
\text { Blu } \\
\text { per } \\
\text { Gallon }\end{array}$ & $\begin{array}{c}\text { Sulfur } \\
\text { Percent } \\
\text { by } \\
\text { Weight }\end{array}$ & $\begin{array}{l}\text { Average } \\
\text { Blu } \\
\text { per } \\
\text { Gallon }\end{array}$ & $\begin{array}{l}\text { Sulfur } \\
\text { Percent } \\
\text { by } \\
\text { Weight }\end{array}$ & & \\
\hline New England & 13,061 & 1.23 & 7.6 & 13,120 & 1.30 & 7.5 & 150,346 & 1.35 & 150.175 & 1.31 & 1,044 & 1,032 \\
\hline Connecticut.. & 13,210 & 55 & 61 & 13,249 & 54 & 63 & 150.115 & 88 & 150.267 & 8t & 1,030 & 1.030 \\
\hline Maine & 40 & - & . & - & - & . & 149.833 & 89 & 1.49 .774 & 90 & .. & - \\
\hline Massachusetts .............. & 12,983 & 1.21 & 8.0 & 13,073 & 119 & 79 & 149.845 & 64 & 149.483 & 159 & 1.045 & $1,0=z$ \\
\hline 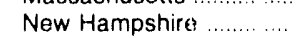 & 13,244 & 2.07 & 70 & 13,187 & 223 & 72 & 154.557 & 192 & 154.523 & 181 & - & - \\
\hline Rhode Island & $\frac{10.24}{20}$ & 2.01 & 2 & 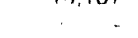 & 20 & . & 150.747 & 100 & 150.684 & 98 & 1.033 & 1,032 \\
\hline Vermont ......... & - & . &. & & - & - & 140.000 & 30 & 138,000 & 30 & 1,000 & - \\
\hline Middle Atlaritic .................. & $12: \because 8$ & 1.91 & 12.4 & 12,460 & 2.01 & 12.6 & 149,147 & .64 & 148,791 & .65 & 1,032 & 1,028 \\
\hline New Jersey ......................... & 13,148 & 115 & 78 & 13,279 & 125 & B: & 148.274 & .13 & 145.594 & 40 & 1,033 & 1,032 \\
\hline 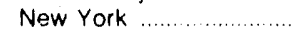 & 12,765 & 1.70 & 9.6 & 12.733 & 171 & 99 & 149.225 & $7 s$ & 149.133 & 75 & 1.031 & 1,027 \\
\hline Pennsylvania & $12,30 \%$ & 203 & 134 & 12.346 & 213 & 134 & 149.416 & 30 & 149.140 & 31 & 1.031 & 1,029 \\
\hline East Nopth Central .......... & 10,995 & 1.87 & 9.2 & 11.081 & 1.88 & 9.1 & 145,265 & .66 & 146,390 & .66 & 939 & 967 \\
\hline Illinoir $\quad \ldots \ldots \ldots \ldots \ldots \ldots$ & 10.678 & 205 & 38 & 10.662 & 2.03 & $\therefore$ & 148.086 & 53 & 148.896 & 61 & 1.016 & 1.021 \\
\hline 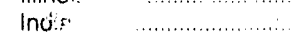 & 10.652 & 2 & 9.2 & 10,763 & 236 & 92 & 136.987 & 33 & .37 .454 & 29 & 921 & 927 \\
\hline Mich & 11,236 & & $7 i$ & 11.440 & 72 & 70 & 144.174 & 75 & 145.967 & $i 7$ & 1.019 & 1.021 \\
\hline 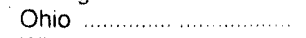 & 11,798 & 2.46 & 117 & 11.871 & 246 & 115 & 146.599 & 81 & 145.147 & 64 & 1.019 & 1,028 \\
\hline Wisconsin ....... & 9.630 & 85 & 6.6 & 9.689 & 89 & 6.6 & 137.569 & 38 & 137.487 & 32 & 1.005 & 993 \\
\hline West North Central & 8,677 & 1.03 & 7.4 & 8,683 & 1.06 & 7.8 & 142,351 & .82 & 143,145 & 1.02 & 985 & 1,000 \\
\hline 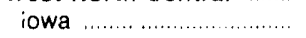 & 8,935 & 76 & 5.9 & 9.163 & 82 & 61 & 137,886 & 43 & 137.474 & 42 & 1.006 & $i, 004$ \\
\hline Kansas ............... & 8,842 & 68 & 6.4 & 8.921 & 74 & 6.5 & 144,284 & 72 & 141,906 & 97 & 980 & 998 \\
\hline Minnesola .......... & 8,703 & 56 & 7.3 & 8.660 & 62 & 80 & 139.948 & 40 & 135.527 & 30 & 1.003 & 1.003 \\
\hline 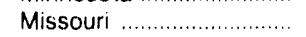 & 10,365 & 2.18 & 8.8 & 10.410 & 2.23 & 90 & 142.979 & 127 & 145.859 & 132 & 1,015 & 992 \\
\hline Nebraska & 8,672 & .36 & 5.2 & 0,585 & 36 & 5.0 & 147.709 & 109 & 147.388 & 121 & 958 & 1,003 \\
\hline North Dakota & 6,589 & 73 & 8.4 & 6,558 & $7 !$ & 9.3 & 138.355 & 41 & 138.912 & 48 & 1.050 & 1,065 \\
\hline 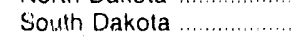 & 6,360 & 84 & 76 & 6,515 & 85 & 73 & 138,050 & 38 & 135.970 & 30 & 996 & 1,002 \\
\hline South Atlan:irs & 12,357 & 1.49 & 10.0 & 12,403 & 1.52 & 10.1 & 150,407 & 1.15 & 150,654 & 1.13 & 1,017 & 1,012 \\
\hline 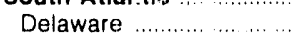 & 12,940 & 1.03 & 9.0 & 13.074 & 151 & 3.1 & 150.014 & 113 & 150.448 & 112 & 1.105 & 1,071 \\
\hline District of Columbia ....... & - & - & - & - & - & . & 143,353 & 91 & 143,650 & 94 & . & - \\
\hline 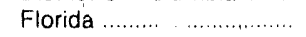 & $\therefore .252$ & 1.75 & 8.6 & 12,250 & 1.77 & 8.7 & 151,208 & 123 & 151,491 & 118 & 1.011 & 1.008 \\
\hline Georgia ...................... & 12,054 & 1.66 & 10.1 & 12.111 & 1.74 & 10.2 & 140,246 & 87 & 144,173 & 1.47 & 1,011 & 1,023 \\
\hline 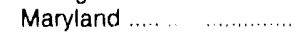 & 12,641 & 1.43 & 10.7 & 12,666 & 1.43 & 11.0 & 149,801 & 1.29 & 149,333 & 1.29 & 1,046 & 1.042 \\
\hline North Carolina .............. & 12,555 & .92 & 9.8 & 12.604 & 92 & 9.0 & 138,530 & .20 & 138,728 & .20 & 1. & - \\
\hline South Carolina .................. & 12,587 & 1.13 & 9.2 & 12.646 & 1.18 & 9.5 & 139,499 & 1.43 & 138.514 & .54 & 1,023 & 1,032 \\
\hline 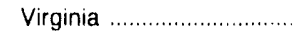 & 12,705 & .92 & 9.3 & 12,804 & 92 & 9.4 & 148,704 & 81 & 147,058 & .83 & 1,042 & 1,054 \\
\hline 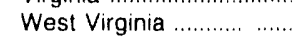 & 12,347 & 1.83 & 11.7 & $12,41 B$ & $i .76$ & 11.7 & 139,312 & 35 & 139,571 & 14 & 1,000 & 1,000 \\
\hline Easi South Central ......... & 11,865 & 2.01 & 10.6 & 11,956 & 1.93 & 10.4 & 146,229 & 1.96 & 145,902 & 1.92 & 1,025 & 1,026 \\
\hline 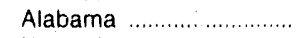 & 11,993 & 1.62 & 11.9 & 12,159 & 1.47 & 11.9 & 135,972 & .25 & 136,477 & .20 & 1,027 & 1,031 \\
\hline 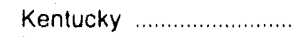 & 11.698 & 2.33 & 10.6 & 11,723 & 2.20 & 10.6 & 138,697 & 26 & 138,657 & .30 & 1,011 & 1,023 \\
\hline Mississippi ......................... & 12,634 & 1.55 & 18 & 12,562 & 1.40 & 7.6 & 149,992 & 271 & 149,754 & 2.71 & 1,025 & 1,026 \\
\hline Tennessee ........................... & 11,828 & 2.06 & 9.6 & 11,986 & 2.08 & 9.6 & 138,175 & .30 & 138,242 & .28 & - & - \\
\hline West South Ceniral ........ & 8,751 & .42 & 6.1 & 8,475 & .43 & 6.1 & 145,730 & .64 & 145,220 & .66 & 1,037 & 1.041 \\
\hline 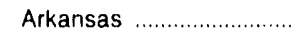 & 8,628 & .33 & 5.0 & 8,550 & 34 & 5.4 & 148,574 & .72 & 143,983 & .63 & 1,016 & 1,029 \\
\hline 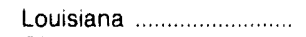 & 8,837 & .50 & 7.8 & 7,961 & .51 & 7.7 & 144,956 & 61 & 146,168 & .68 & 1,033 & 1,044 \\
\hline 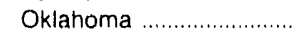 & 8,777 & .43 & 5.5 & 8,847 & .43 & 5.3 & 140.062 & .51 & 138,765 & .52 & 1,047 & 1,038 \\
\hline 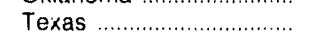 & 7,229 & .74 & 12.2 & 7,248 & .70 & 12.2 & 142,833 & .54 & 144,556 & .56 & 1,033 & 1,034 \\
\hline 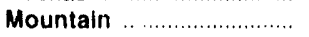 & 9,859 & .56 & 11.2 & 9,829 & .56 & 10.9 & 143,753 & .62 & 113,284 & .65 & 1,028 & 1,020 \\
\hline 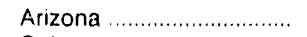 & 10,591 & .50 & 11.4 & 10,663 & .51 & 11.1 & 143,649 & .53 & 139,328 & .30 & 1,036 & 1,034 \\
\hline 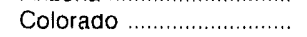 & 9,861 & .37 & 6.9 & 9,792 & .41 & 7.0 & 136,950 & .34 & 138,812 & .53 & 952 & 963 \\
\hline 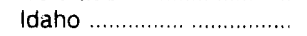 & - & - & - & - & - & - & - & - & - & - & - & - \\
\hline 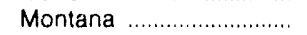 & 8,513 & .66 & 9.5 & 8,522 & .67 & 9.4 & 136,865 & .50 & 118,412 & 42 & 1,317 & 1,208 \\
\hline Nevada & 11,877 & .52 & 9.3 & 11,963 & .50 & 9.3 & 147,363 & .77 & 147,611 & 69 & 1,032 & 1,031 \\
\hline 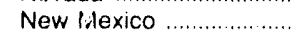 & 9,213 & .84 & 21.7 & 9,092 & .81 & 21.4 & 136,052 & .83 & 138,732 & 1.87 & 1,036 & 1,028 \\
\hline 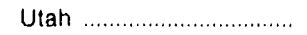 & 11,406 & .49 & 11.3 & 11,592 & .50 & 10.7 & 138,328 & .24 & 138,722 & .47 & 875 & - \\
\hline 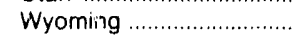 & 8,785 & .53 & 8.0 & 8,709 & .53 & 7.9 & $139,35 i$ & .30 & 139,509 & .32 & 1,000 & 1,000 \\
\hline Pacific Contiguous .......... & 8,083 & .63 & 13.1 & 8,179 & .64 & 13.9 & 146,166 & .30 & 146,993 & .34 & 1,039 & 1,035 \\
\hline California .... ................... & - & - & - & - & - & - & 146,223 & .29 & 147,060 & 34 & 1.039 & 1,035 \\
\hline 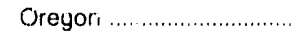 & 7,977 & .34 & 4.5 & - &.. & - & 138,800 & .05 & - & - &.. & - \\
\hline Washingtain & 8,088 & .65 & 13.6 & 8,179 & .64 & 13.9 & $i 41,447$ & 1.55 & 141,000 & .30 &.. & - \\
\hline Pacific Moncontlguous & 7,909 & .15 & 8.2 & 7,819 & .14 & 8.7 & 148,935 & .64 & 149,387 & .62 & - & - \\
\hline Alasina & 7,909 & .15 & 8.2 & 7,819 & .14 & 8.7 & 134,283 & .20 & - & - & - & - \\
\hline Hawali .................................. & - & - & - & - & - & - & 148,937 & .64 & 149,387 & .62 & - & - \\
\hline U S. Averago & 10,428 & 1.33 & 10.0 & 10,459 & 34 & 9.9 & 149,260 & .93 & 149,309 & .93 & 1,030 & 1,032 \\
\hline
\end{tabular}

- Data for 1989 are preliminary

- Data for 1988 are revised; See Appendix $C$ for an explanation.

Notes. Totals may not equal sum of compcnents because of independent rounding. - Data include petroleum coke.

Source Energy Information Administration, Form El/ 767. "Steam-Electric Plant Cperation and Dosign Report." 
Table 47. Average Flue Gas Desulfurization Costs by Census Division and Statk 1986-1989

\begin{tabular}{|c|c|c|c|c|c|c|c|c|}
\hline \multirow{2}{*}{$\begin{array}{l}\text { Census Division } \\
\text { and State }\end{array}$} & \multicolumn{4}{|c|}{$\begin{array}{l}\text { Average Osm Costs } \\
\text { (Mills per Kllowatthour) }\end{array}$} & \multicolumn{4}{|c|}{$\begin{array}{l}\text { Average Installed Cost } \\
\text { (Dollars per Kllowatt) }\end{array}$} \\
\hline & $1989^{\prime}$ & 1988 & 1987 & 1988 & $1989^{\prime}$ & 1988 & 1987 & 1986 \\
\hline New England ............................ & - & - & - & - & - & - & - & - \\
\hline 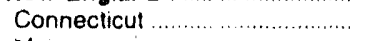 & - & - & - & - & - & - & - & - \\
\hline Maine & - & - & - & - & - & - & - & 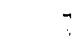 \\
\hline Massachusetts & - & - & - & - & - & - & - & $\therefore$ \\
\hline New Hampshire & - & - & - & - & - & - & - & - \\
\hline Rhode Island & - & - & - & - & - & - & - & - \\
\hline Vermont & - & - & - & - & - & - & - & - \\
\hline MidG de Atlantic & 4.92 & 4.15 & 4.78 & 5.19 & 175 & 176 & 175 & 183 \\
\hline 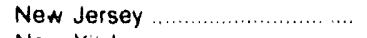 & 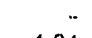 & & - & - & - & - & - & - \\
\hline New York & 1.24 & 1.23 & 1.28 & 1.33 & 223 & 223 & 223 & 223 \\
\hline Pennsylvania & 5.90 & 4.83 & 5.66 & 6.10 & 168 & 170 & 168 & 177 \\
\hline East North Central ................... & 2.41 & 2.36 & 2.49 & 2.65 & 137 & 139 & 139 & 139 \\
\hline Illinios & 2.69 & 2.64 & 2.50 & 2.72 & 146 & 146 & 145 & 145 \\
\hline Indiana & 2.26 & 2.26 & 2.36 & 2.39 & 153 & 157 & 157 & 157 \\
\hline 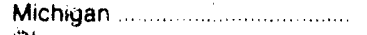 & - & - & - & & - & - & - & - \\
\hline Ohio & 2.76 & 2.39 & 3.58 & 4.44 & 47 & 47 & 47 & 47 \\
\hline Wisconsin & - & - & - & - & - & - & - & - \\
\hline West North Central & .73 & .80 & .86 & .68 & 74 & 72 & 71 & 71 \\
\hline lowa' $\ldots$ & 2.30 & 2.03 & 2.36 & 2.57 & 222 & 222 & 153 & 153 \\
\hline$\ldots \ldots \ldots \ldots \ldots \ldots \ldots$ & .59 & .59 & 29 & .18 & 71 & 72 & 71 & 71 \\
\hline Minnesota & 26 & .30 & .38 & .40 & 74 & 70 & 70 & 70 \\
\hline . & 2.05 & 1.78 & 2.36 & 2.01 & 89 & 89 & 82 & 82 \\
\hline 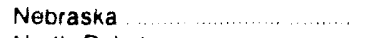 & - & - & - & - & - & - & - & - \\
\hline North Dakota ......................... & $\$ 1$ & 1.13 & .98 & .77 & 65 & 59 & 59 & 59 \\
\hline South Dakota $\ldots \ldots$ & - & & - & - & - & - & - & - \\
\hline South Atlantic & 1.47 & 1.96 & 1.63 & 1.79 & 92 & 93 & 92 & 111 \\
\hline Delaware ${ }^{t}$ & 30.80 & 35.81 & 25.61 & 23.50 & 1,385 & 1,385 & 1,385 & 1,385 \\
\hline District of Columbia .............. & - & & - & - & - & - & - & - \\
\hline Florida $\ldots$ & 84 & 1.60 & 1.20 & 1.28 & 81 & 81 & 81 & 126 \\
\hline$\ldots \ldots \ldots \ldots$ & - & - & - & - & - & - & - & - \\
\hline$\ldots \ldots+\cdots$ & - & - & - & - & -. & - & - & - \\
\hline North Carolina & - & - & - & - & - & - & - & - \\
\hline South Carolina & 61 & 59 & 59 & .87 & 46 & 46 & 46 & 46 \\
\hline Virginia ....... & - & & - & - & - & - & - & - \\
\hline West Virgina $\ldots$ & 2.11 & 2.14 & 1.91 & 1.78 & 111 & 111 & 110 & 98 \\
\hline Last South Central $\ldots$ & 1.48 & 1.66 & $i 61$ & 1.66 & 123 & 122 & 121 & 121 \\
\hline Alabama & .76 & 1.58 & 1.13 & 1.30 & 82 & 82 & 82 & 82 \\
\hline$\ldots+\ldots+\ldots$ & 1.73 & 1.75 & 1.81 & 1.86 & 137 & 137 & 137 & 137 \\
\hline Mississippi & 33 & .29 & .40 & .27 & 70 & 70 & 70 & 70 \\
\hline ................ & - & - & - & - & - & - & - & - \\
\hline West South Central $\ldots$ & .99 & .93 & 1.08 & 1.04 & 70 & 69 & 72 & 72 \\
\hline Aikansas & - & - & 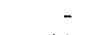 & - & - & $\because$ & $=$ & - \\
\hline ……............. & - & .13 & 11 & .12 & 75 & 75 & 75 & 75 \\
\hline Oklahioma & 54 & 57 & 1.29 & 64 & 92 & 92 & 92 & 92 \\
\hline Texas $\ldots \ldots \ldots$ & 1.03 & 1.03 & 1.15 & 1.14 & 68 & 67 & 70 & 70 \\
\hline ..................... & 71 & .74 & 39 & .99 & 152 & 152 & 153 & 152 \\
\hline … & .71 & 1.05 & 1.03 & 1.29 & 141 & 141 & 123 & 123 \\
\hline Colorado & .49 & .49 & .56 & .44 & 85 & 85 & 85 & 85 \\
\hline Idaho $\ldots$ & - & - & . & - & - & - & - & - \\
\hline Montana & 1.04 & .80 & 1.34 & 1.02 & 274 & 274 & 274 & 274 \\
\hline 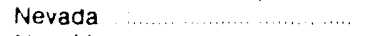 & 1.51 & 1.80 & 1.68 & 4.45 & 126 & 126 & 126 & 120 \\
\hline New Mexico & 91 & 1.04 & 1.35 & 1.32 & 192 & 192 & 192 & 192 \\
\hline Utah & .52 & .49 & .64 & .75 & 97 & 97 & 89 & 72 \\
\hline Wyoming & .56 & 62 & .49 & .62 & 137 & 137 & 137 & 137 \\
\hline Pacific Contiguous & - & - & - & - & - & - & - & - \\
\hline Californı & - & - & - & - & - & - & - & - \\
\hline Oregon & - & - & - & - & - & - & - & - \\
\hline Washington & - & - & - & - & - & - & - & - \\
\hline Pacific Noncontiguous ............. & - & - & - & - & - & - & - & - \\
\hline Alaska & - & - & - & - & - & - & - & - \\
\hline Hawair & - & - & - & - & - & - & - & - \\
\hline U.S. Average & 1.34 & 1.35 & 1.50 & 1.61 & 118 & 119 & 119 & 121 \\
\hline
\end{tabular}

'Data for 1989 are preliminary.

- The high cost shown for Delaware is attributable to the flue gas desulfurization (FGD) units belonging to a plant that provides steam for sale and steam used to produce eiectricity. The FGD costs include the costs incurred in the production of steam for sale.

$O \& M=$ Operation and Maintenance

Notes: - Totals may not equal sum of components because of independent rounding. - Data include petroleum coke. $A$ mill is a monetary cost and billing unit equal to $1 / 1000$ of the U.S. dollar (equivalent to $1 / 10$ of 1 cant).

Source: Energy Information Administration, Form ElA-767, "Steam-Electric Plant Operation and Design Report." 


\section{Electric Power Transactions}

This chapter provides summary information for the electric utility industry on its operations and wholesale electricity trade at the international (Canada and Mexico), national, and North American Electric Reliability Council (NERC) region levels. The NERC is an organization established by the electric utility industry for maintaining, coordinating, and promoting reliability among the interconnected systems of North America.

\section{Background}

An electric power system is a group of generation, transmission, distribution, communication, and other facilities that are physically connected and operated as a single unit under one control (Figure 23). Transmission and distribution lines and associated facilities are used to transmit electricity from its point of origin the generator) to the ultimate consumer, Although, due to its physical characteristics, electricity flows along all available paths, it follows the path of least resistance. The flow of electricity must be closely monitored to ensure that a suthicient quantity is available to satisfy all demand (load) for electricity placed on the power system. In addition, the flow is maintained at a fre. quency of 6() hertz per second.

The flow of electricity within the system is maintained and monitored by dispatch centers. The dispatch center must inventory and prioritize all generating capacity a vailable to it, track transactions involving the buying or selling of either electric power or capacity, monitor current load, and anticipate future load on the system. It is the responsibility of the dispatch center to match the supply of electricity with its demand. The demand for electricity is not constant in nature. That is, load requirements fluctuate continuously, based on such factors as time of day, season of the year, and the characteristics of territory served by the system. Nonetheless, the dispatch center must be ready to meet the highest level of load placed on the system. The dispatch center must accommodate the loss of generating facilities (both when planned and when unexpected). In addition, the center must monitor transmission lines to determine whether the flow of electricity is approaching the carrying limits of the lines. In order to carry out its responsibilities in a timely fashion, the dispatch center is authorized to buy and sell electricity based on system requirements.

Authority for these transactions has been preapproved under interconnection agreements (contracts) that have been signed by all the electric utilities that are physically interconnected. These transactions include purchases, sales for resale, exchanges, and whe. ling of energy. Purchase transactions involve buying power among electric utilities and nonutility producers of electricity. Sales for resale transactions refer to power sold by one electric utility to other utilities for distribution. Some transactions involving the trade of electric energy are based on the availability of excess generating capacity or the diversity in load requirements. For example, if one electric utility has its lowest load during the winter season, it may arrange to offer its available excess generating capacity in exchange for excess generating capacity available at a facility with low summer load. This type of arrangement is an exchange transaction. However, the repayment or replacement of exchange energy may extend over several years. Wheeling transactions are the movement of elec:tricity from one utility to another utility over the transmission facilities of the intervening utilities.

\section{Power Pool Transactions}

In addition to dealing in one-time purchase and sale transactions, many electric utilities have joined together and formed power pools to achieve better operating efficiencies and to gain additional support for maintaining a functional electrical system. Thus, they share the benefits achieved by joint planning, coordinated use of generating and transmission facilities, and/ or coverage of facility outages. This coordination also provides the opportunity to manage short-term saving, much of it coming from varying fuel prices and the costs associaled $w: t h$ different mixes of capacity.

Power pools can be made up of two electric utilities, like the Michigan Flectric Coordinated System (Detroit Edison Company and Consumer Power Company), include all the major investor-owned utilities within a State (the New York Power Pool), or cross State lines (the PJM Power Pool includes parts, or all of Pennsylvania, New Jersey, Maryland, and Delaware). 
Figure 23. An Integrated Electric Utility System

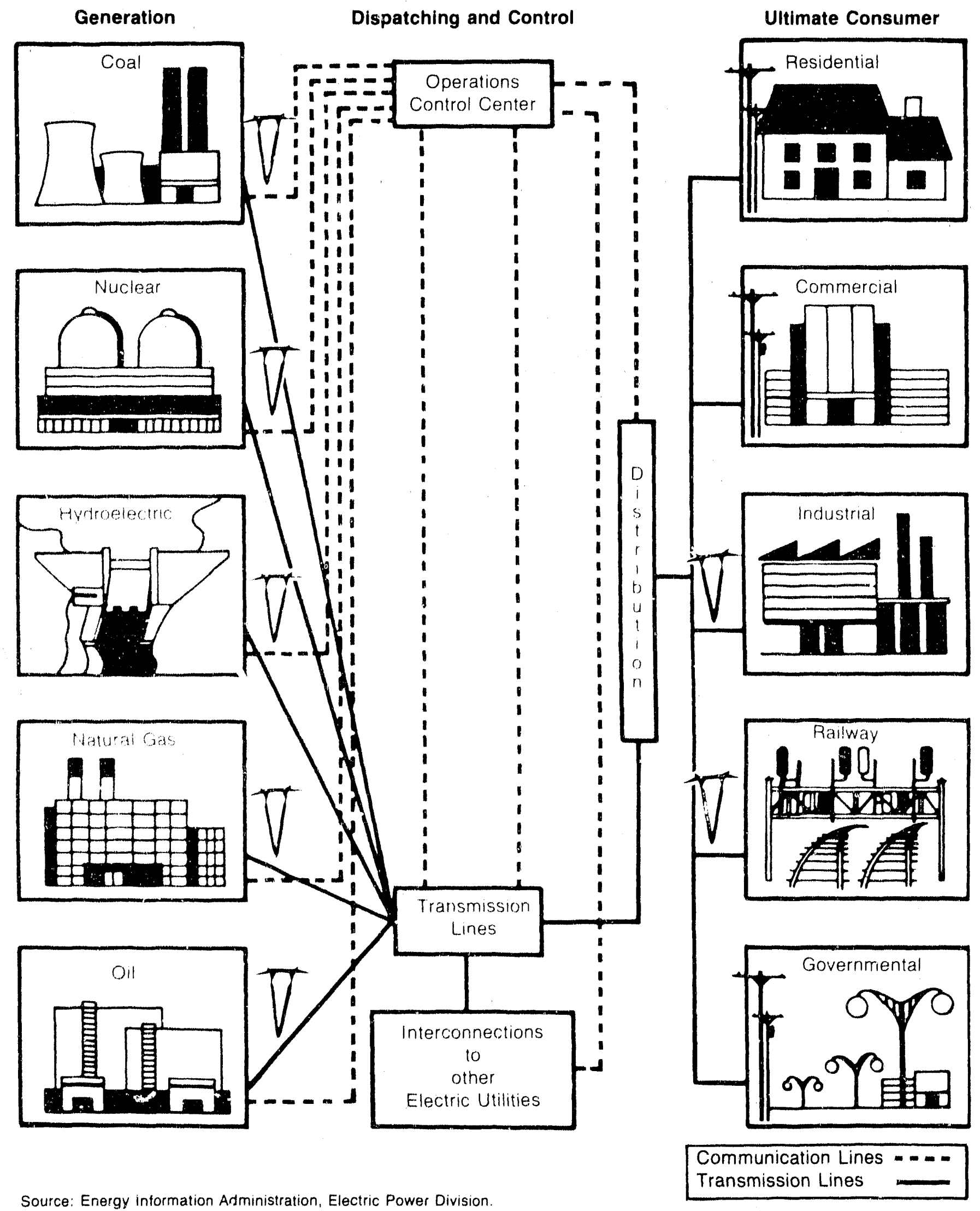


Power pools may run under a single-system dispatch to meet combined-load requirements and naintenance programs, or just share the benefits of planned or hourly wholesale sales of power and energy among the member utilities. Power pools may have responsibility for coordinating flow within the geographic area of the interconnected systems. In any case, they are bound by the operating standards established by the electric power industry. These standards require the coordination and maintenance of system stability and reliable service on a regional basis.

\section{NERC Profile}

The North American Electric Reliability Council (NERC) was formed in 1968 during the aftermath of the Northeast Blackout on November 9, 1965, and be- came incorporated in 1975 (Figure 24). The NERC consists of nine regional reliability councils whose memberships comprise essentially all of the electric utility systems in the contiguous United States, Canada, and Baja California Norte, Mexico. The NERC also has one affiliate member, the Alaska Systems Coordinating Council. The regional councils are responsible for maintaining the three power grids (the Seven Interconnected Regions Power Grid or the Eastern Power Grid, tt: - w ustern Power Grid, and the Electric Reliability Council of Texas Power Grid) present in the contiguous United States. The data for NERC regions in this publication are based upon the assignment of all electric utilities to an individual region and are for the U.S. portion of the regions only.

Figure 24. North American Electric Reliability Council Region Map for the United States

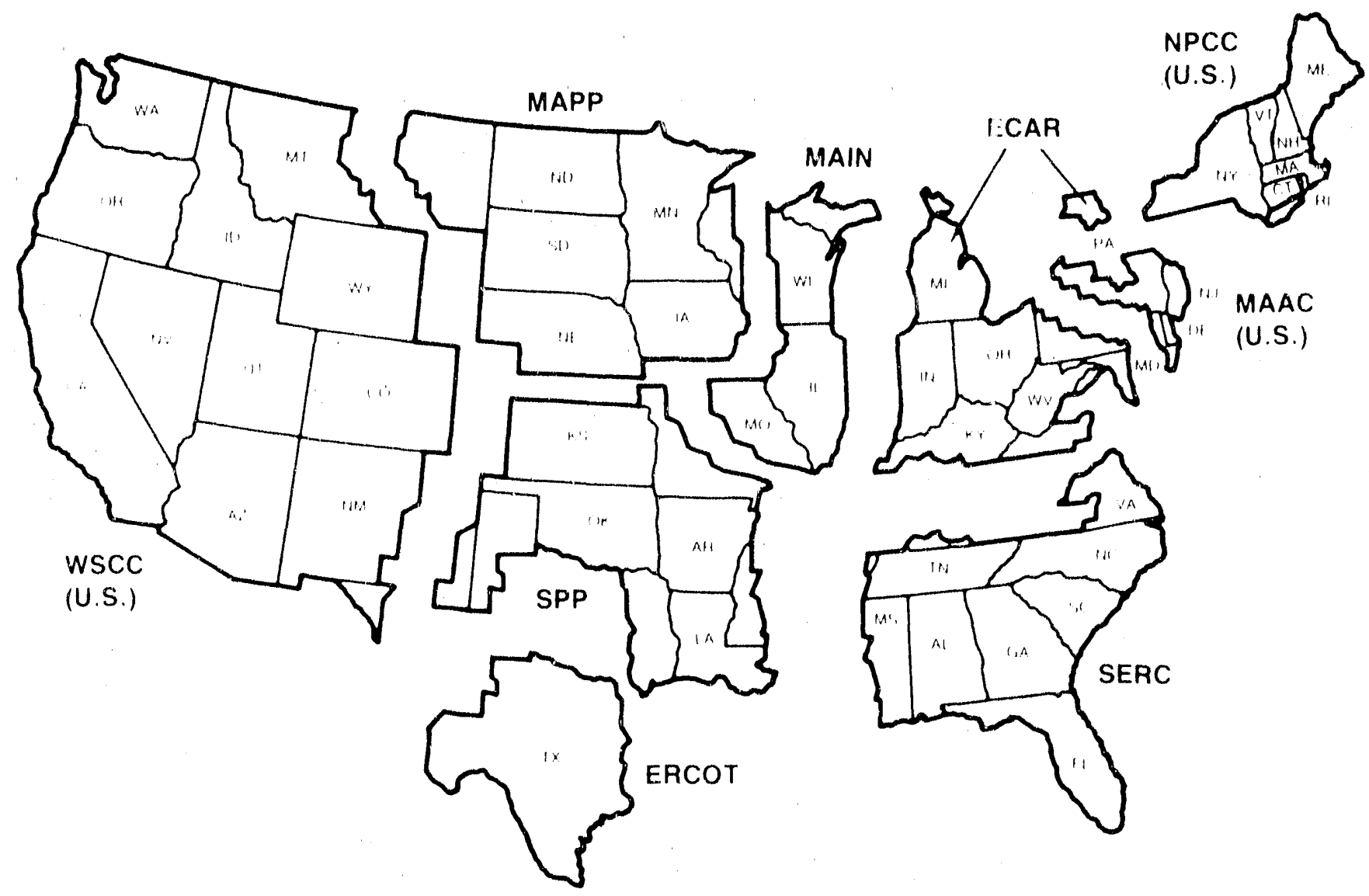

Regional Electric Reliability Council Areas:

ECAR - East Central Area Reliability
Coordination Agreement
MAIN-Mid-American Interpool
Network
MAAC- Mid-Atlantic Area Council
MAPP (U.S.) - Mid-Continent Area Power
Pool
NPCC (U.S.) - Northeast Fower Coordinating
Councii

\author{
SERC-Southeastern Electric \\ Reliability \\ Council \\ SPP_-Southwest Power Pool \\ ERCOT-Electric Reliability Council of \\ Texas \\ WSCC (U.S.)-Western Systems Coordinating \\ Council
}




\section{Electric Utility Transactions}

Electric utility transactions (wholesale electricity irade) include purchases, sales for resale, exchanges, and the transmission of electricity. These transactions allow electric utilities to share resources and to provide mutual assistance in time of potential and actual need. They allow the utility systems to provide lower cost service to their consumers by taking advantage of the load diversity of each utility. These transactions also allow each utility to conserve its own resources, to share the benefits of reduced operating costs with iow consumers, to receive emergency energy support from, other utilities, and to reduce the cost of its own requirements for operating reserve. However, due to the complexity of electric power transactions involving the specifics of contracts, simultaneous energy transactions, the unintended receipt and delivery of energy (inadvertent flow), and losses, the reporting of both the classification and quantity of each transaction among utilities may not be consistent.

Electric utilities originally became interested in energy transactions hecause of the savings gained from reduced or avoided production costs. An electric utility can avoid building expensive additional capacity if the electricity can be obtaned from other sources. Purchasing power from other utilities can help a utility meet peak load without using expensive oil- or gas-lired turbines. Similarly, a utility benefits from heing able to delay or stagger construction of additional haseload plants. Electric utilities can also delay new plant construction by purchasing electricity from nonutility gencrators under long-term contracts.

\section{Regulation of Electric Utility Transactions}

The Federal Fnergy Regulatory Commission (FIRC) is responsible for regulating wholesate transactions. Flectric utilities file with the FERC for approval of proposed rate schedules for transmission services and charges, and for wholesale transactions. Transmission filings cover the allocation of electric power flows on the transmission line systerns. These filings cover firmcarrving capacity, interruptible-carrying capacity, and thira-party-iransfer capacity. Other categorices of filings usually cover the responsibilities of the utilities to one another during normal and emergency conditions, operating-reserves support, diversity exchanges, and unscheduled or inadvertent-energy flows.

Wholesale transactions include capacity sales, energy sales, and energy exchanges. Wholesale transactions are further divided by duration of the sale and the type of capacity and energy sold. The length of the sale can be for an hour, a day, a week, a month (or several months), a season, several years, or some combination of these time periods.

Capacity sales are usually considered firm sales (that is, associated energy may be taken, or the capacity must be paid for if the energy is not taken; and the delivery is scheduled during normal system operating conditions). This capacity may be made available from the entire system or from an identified generating unit. The capacity offered in these transactions may be available only during a set period of seasonal diversity, an off-peak time of the day, or from a generator, which is corrently not fully utilized, that is fired by a particular fuel. The energy assuciated with this capacity sale, if required, has a separate cost schedule from the capacity charge attached to each kilowatt of power.

Nonfirm sales, sometimes called energy, economy, or interruptible sales, do not have a demand or capacity charge included in the price of the transaction. These transactions are subject to curtailment or cessation of delivery by the supplier in accordance with prior agreements or under specified conditions. The sales are often based on splitting the benefits gained by the parties involved. They are used to gain operational savings, such as avoiding the use of more expensive fuels, and selling electricity genterated by the spillage of excess reservoir water.

Energy exchanges involve transfers of energy to other systems at no monetary charge. The energy must be returned in kind at a later date agreed upon by both partices. Otherwise, the receiving party pays for the energy received. The incidental miscellaneous transfer of energy and inatvertent flow are also handled in the same manner. In total, these wholesale transactions have hecome very important tools used hy the U.S. electric utility industry to reduce costs and avoid expensive new capacity.

\section{International Transactions}

U.S. utilitics have also taken advantage of heing able to enter into international trade agreements to acquire energy from Canada and Mexico. These trads agreements between utilities cover a variety of Iransatetion options. These options include purchasing axofirm energy from relatively inexpensive renewable resources (hydroelectric from Canada and geothermal from Mexico); acquiring additional generating capability to support the requirements for supply at U.S. electric utilities; the holding of purchased electricity (as reservoir water) to be reacquired when needed by U.S. electric utilities; and sharing the benefits of coordinated operations planning for the systems. In some instances, consumers can be served more efficiently if they are connected to foreign transmission lines, because they are geographically closer to those lines. 
it A $P$ 
Table 48. Energy Account Balance of Electricity, 1987-1989

(Million Kilowatthours)

\begin{tabular}{|c|c|c|c|}
\hline item & $1989^{\prime}$ & 1988 & 1987 \\
\hline Source & & & \\
\hline Net Generation ............... & $2,811,398$ & $2,730,774$ & $2,632,019$ \\
\hline 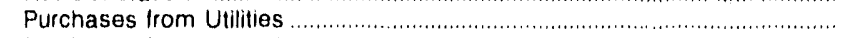 & 959,375 & 922,038 & 870,345 \\
\hline 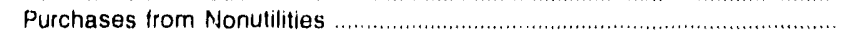 & 89,813 & 68,030 & 50,165 \\
\hline 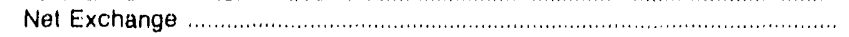 & 2,369 & 32,955 & 45,619 \\
\hline 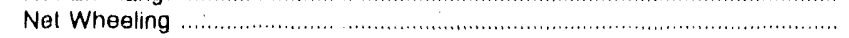 & 3,525 & 2,262 & 2,019 \\
\hline \multicolumn{4}{|l|}{ Disposition } \\
\hline Sales to Ultimate Consumers. & $2,646,809$ & $2,578,062$ & $2,457,272$ \\
\hline Sales for Resale & 975,676 & 945,993 & 916,703 \\
\hline Energy Furnished Without Charge & 5,647 & 5,525 & 5,149 \\
\hline 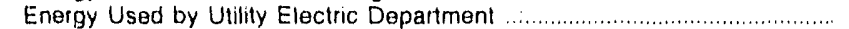 & 19,501 & 16,962 & 16,965 \\
\hline Energy Losses ${ }^{3}$ & 218,846 & 209,516 & 199,861 \\
\hline
\end{tabular}

' Data for 1989 are preliminary

2 The shift in magnitude for net axchange reflects a change in accounting procedures by electric utilities because of reclassification of various transactions for bulk trade. In addition, the Tennessee Valley Authority reduced their net exchanges by 15 billion kilowatthours from 1988.

3 These values are not measured; however, they represent losses and unaccounted for energy. These values are calculated in order that source and disposition of energy are equivalent.

Notes: Annuil net generation data shown here should only be used in comparison with other Form ElA.861 data. Tolal annual net generation data are published by the Energy information Administration from its Forin E:IA.758. "Monthly Power Plant Report," (Table 1). •Totals may not equal sum of iomponents because of independerit rounding. - Source and disposition are the aggregation of data reported lor each utility. Source and disposition of energy are not additive due to double counting. Purchases from utilities, nel exchange and nel wheeling (except for imports) are included in net generation. Sales for resale is included in sales to vitimate consumers. Due to the complexity of electric power transactions that involve specifics of contracts, simultaneous energy transactions, the unintended receipt and delivery of onergy (inadvertent flow), and losses. uniformity in reporting the classification and quantity of each transaction amiong utilities may nol exist.

Source: Energy Information Administration, Form E.A.861. "Annual Electric Utility Report."

Table 49. Net Generation by North American Electric Reliability Council Region, Alaska, and Hawaii, 1987-1989

(Million Kilowatthours)

\begin{tabular}{|c|c|c|c|c|}
\hline $\begin{array}{c}\text { North American Electric } \\
\text { Rellabllity Council Region, } \\
\text { Alaska, and Hawall }\end{array}$ & $1989^{\prime}$ & 1988 & & 1987 \\
\hline ELAR & 487.659 & 487,040 & & 463,569 \\
\hline$\ldots \therefore-\ldots-1, \ldots$ & 185,011 & 179,442 & . & 173,645 \\
\hline$\ldots+\ldots \ldots$ & 193,889 & 191,874 & & 185,541 \\
\hline$\ldots \ldots \ldots$ & 200,660 & 198,666 & & 185,240 \\
\hline$\ldots \ldots \ldots$ & 116,410 & 118,238 & & 122,381 \\
\hline$\ldots \ldots \ldots \ldots$ & 233,624 & 223.996 & & 212,165 \\
\hline SEAC & 612,643 & 571,541 & & 559,700 \\
\hline SPP & 239,032 & 233,396 & & 231,865 \\
\hline WSCCOUS.) & 530,205 & 514,622 & & 486.398 \\
\hline Contiguous U.S. & $2,799,130$ & $2,718,815$ & & $2,620,505$ \\
\hline Alaska & 4,575 & 4,460 & & 4,412 \\
\hline$\ldots \ldots$ & 7.692 & 7,499 & & 7,103 \\
\hline (., & $2,811,398$ & $2,730,774$ & & $2,632,019$ \\
\hline
\end{tabular}

- Data for 19999 are preliminary

Notes: Annual net generation data shown here should only be used in comparison with other Form ElA-861 data. Total annual nel generation data are published by the Enigrgy Information Adrninisiration trorn its Form E.IA.759. "Monthly Power Plant Report," (Table 1). •Totals may not equal sum of com;onents because of independent rounding.

Source: Lnergy Intormation Administration, Form EIA.861, "Anriual Elactric Utility Report." 
Table 50. Sales to Ultimate Consumers by Sector, North American Electric Rellability Council Region, Alaska, and Hawail, 1987-1989

(Million Kilowatthours)

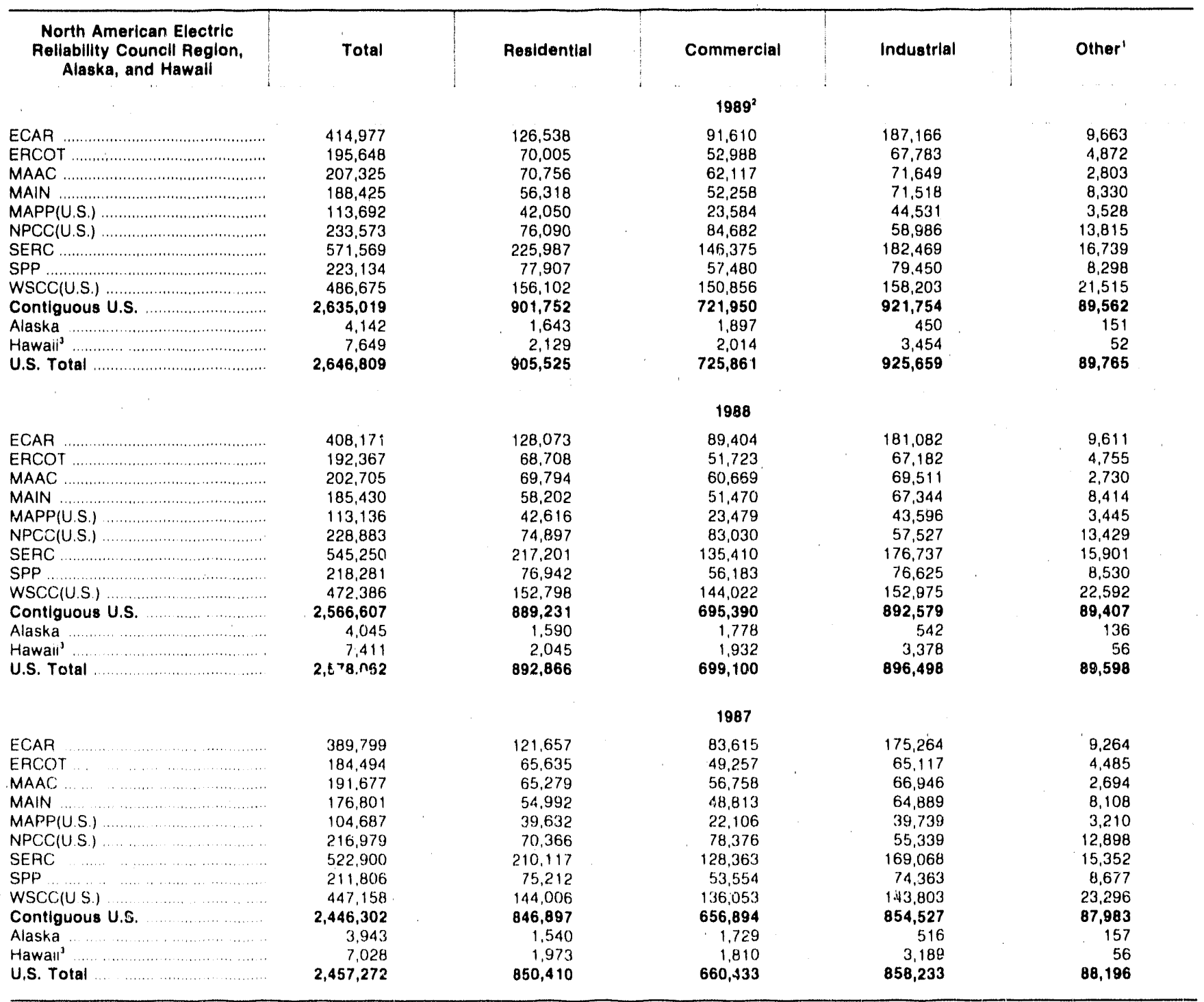

1 includes public street and highway lighting, other sales to public authorities, sales to railroads and railways, and interdepartmental sales.

- Data lor 1989 are preliminary.

3 Hawall includes nonutility transactions only

Note: Totais may not equal sum of components because of independent rounding

Source: Energy Information Administration, Form EIA-861, "Annual Electric Utility Report." 
Table 51. Generating Capability by North American Electric Reliability Council Region, Alaska, and Hawail, as of December 1987-1989

(Megawatts)

\begin{tabular}{|c|c|c|c|}
\hline $\begin{array}{l}\text { North American Electric } \\
\text { Rellabllity Council Region, } \\
\text { Alaska, and Hawall }\end{array}$ & 1989 & 1988 & 1987 \\
\hline 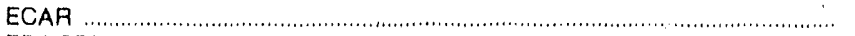 & 102,545 & 101,257 & 101,337 \\
\hline ERCOT & 49,733 & 48,928 & 45,835 \\
\hline MAAC & 50,071 & 48,766 & 48,744 \\
\hline 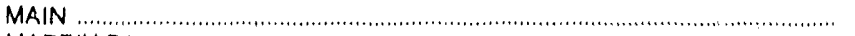 & 49,511 & 49,631 & 49,228 \\
\hline MAPP(U.S.) & 30,376 & 30.348 & 32.718 \\
\hline SERC & 145,129 & 142,404 & 141,430 \\
\hline SPP & 70,468 & 70,311 & 70.158 \\
\hline 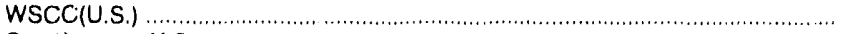 & 129,296 & 128,986 & 128,112 \\
\hline Contiguous U.S. & 681,589 & 674,697 & 671,293 \\
\hline 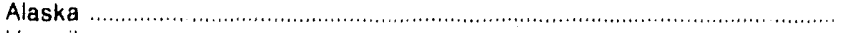 & 1,546 & 1.504 & 1,490 \\
\hline 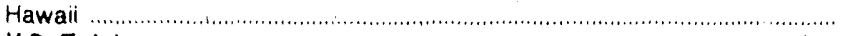 & 1,483 & 1,453 & 1,361 \\
\hline 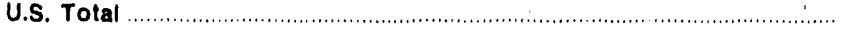 & 684,619 & 677,654 & 674,144 \\
\hline
\end{tabular}

Noles: - Totals may not equal sum of components because of independent rounding. - Generating capability is net summer capabitity

Source: Energy Information Administration, Form EIA.860, "Annual Electric Generator Report."

Table 52. Noncoincidental Peak Load by North American Electric Reliability Council Region, Alaska, and Hawail, 1987-1989

(Megawatts)

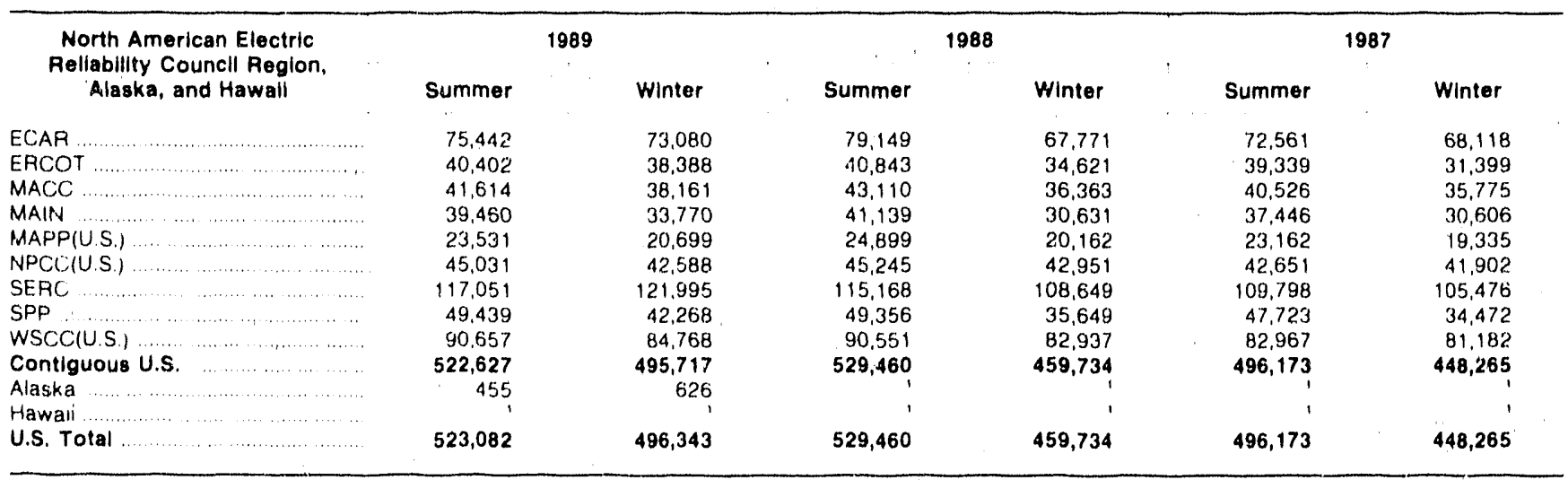

' Data for Alaska were first submitted in 1989; data for Hawall are nol submitted for this form

Note: Noncoincidental peak load is the sum of utility peak loads that may occur at the same time.

Source. Department of Energy, Oftice of the Assistant Secretary for International Affairs and Energy Emergencies, Form IE.411., "Coordinated Butik Power Supply Program." 
Table 53. Utility Receipts by North American Electric Rellability Council Region, Alaska, and Hawail, 1987-1989

(Million Kilowatthours)

\begin{tabular}{|c|c|c|c|c|c|c|c|c|c|c|c|c|}
\hline \multirow{3}{*}{$\begin{array}{c}\text { North American } \\
\text { Electric } \\
\text { Rellability } \\
\text { Council Region, } \\
\text { Alaska, and } \\
\text { Hawail }\end{array}$} & \multicolumn{3}{|c|}{$\begin{array}{c}\text { Total } \\
\text { Recelpts' }\end{array}$} & \multicolumn{3}{|c|}{$\begin{array}{l}\text { Purchased } \\
\text { Power }\end{array}$} & \multicolumn{3}{|c|}{$\begin{array}{l}\text { Exchange } \\
\text { Recelved }\end{array}$} & \multicolumn{3}{|c|}{$\begin{array}{l}\text { Wheellng } \\
\text { Recelved }\end{array}$} \\
\hline & 1 & , & , & 1 & ! & $i$ & & & .. & & & \\
\hline & $1989^{2}$ & 1988 & 1987 & $1989^{2}$ & 1988 & 1987 & $1989^{2}$ & 1988 & 1987 & $1989^{2}$ & 1908 & 1987 \\
\hline ECAR & 206,000 & 186,112 & 161,099 & 90,953 & 95,143 & 84,289 & 93,949 & 75,848 & 66,463 & 21,098 & 15,121 & 10,347 \\
\hline ERCOT & 102,501 & 101,642 & 96,724 & 58,354 & 58,629 & 54,710 & 34,733 & 1.153 & 35,482 & 0,414 & 7,861 & 6,532 \\
\hline MAAC & 86,267 & 76,386 & 70,874 & 46,371 & 41,173 & 32,381 & 31,511 & i./.131 & 31,827 & 8,385 & 7,482 & 6,665 \\
\hline MAIN .. & 63,790 & 58,756 & 56,675 & 33.699 & 29,756 & $28,2.51$ & 28,877 & 27. & 26,353 & 1,215 & 1,915 & 2,071 \\
\hline 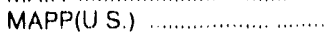 & 114,246 & 114,754 & 105,209 & 68,948 & 68,937 & 62,757 & 36,090 & 38 & 35,078 & 9,208 & 7,804 & 7,374 \\
\hline 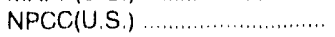 & 183,999 & 178,664 & 159,536 & 125,993 & 122,867 & 121,912 & 15,109 & 17,432 & 17,139 & 42,897 & 38,365 & 20,486 \\
\hline SERC & 383,664 & 348,524 & 339,525 & 278,755 & 256,746 & 243,312 & 82.183 & 79,424 & 85,174 & 22,726 & 12,354 & 11,039 \\
\hline SPP & 140,237 & 130,316 & 116,128 & 107,120 & 102,211 & 89,680 & 13,526 & 8,015 & 16,292 & 19,591 & 20,089 & 10,156 \\
\hline WSCC(U.S.) & 485,872 & 443,345 & 405,057 & 236,294 & 211,960 & 200,690 & 84.754 & 73,630 & 85,411 & 164.824 & 157,755 & 118.925 \\
\hline Contiguous U.S. & $1,766,575$ & $1,638,499$ & $1,590,828$ & $1,046,488$ & 987,422 & 917,984 & 420,730 & 382,332 & 399,249 & 299,356 & 268,746 & 193,594 \\
\hline Alaska. & 2,746 & 2,607 & 2,536 & 2,253 & 2,154 & 2,055 & 28 & 28 & 27 & 465 & 424 & 455 \\
\hline Hawaii $^{3}$.. & 452 & 497 & 481 & 446 & 492. & 471 & 6 & 5 & 11 & 0 & 0 & 0 \\
\hline U.S. Total & $1,769,773$ & $1,641,604$ & $1,513,845$ & $1,049,187$ & 990,068 & 920,509 & 420,764 & 382,365 & 399,286 & 299,822 & 269,170 & 194,049 \\
\hline
\end{tabular}

' Equals purchased power plus exchange received plus wheeling received

2 Data for 1989 are preliminary.

3 Hawall includes nonutility transactions only

Notes. Totals may not equal sum of components bocause of independent rounding. - Summation of utility trade for utilities that operate within the NEAC Fegion. Due to the complexity of electric power transactions that involve specifics of contracts, simultaneous energy tansactions, the unintonded teceipt and delivery of energy (inadvertent flow), and losses, uniformity in reporting the classification and quantity of each transection among utilities may not exist. Includes uflity, import, export, and nonutility transactions.

Source: Energy Inlormation Administration, Form EIA-861. "Annual Electric Utility Report."

Table 54. Utility Deliveries by North American Electric Reliability Council Region, Alaska, and Hawaii, 1987-1989

(Million Kilowatthours)

\begin{tabular}{|c|c|c|c|c|c|c|c|c|c|c|c|c|}
\hline \multirow{2}{*}{$\begin{array}{l}\text { North American } \\
\text { Electric Rellablilty } \\
\text { Councll Region, } \\
\text { Alaska, and Hawail }\end{array}$} & \multicolumn{3}{|c|}{$\begin{array}{c}\text { Totw: } \\
\text { Dellveries }\end{array}$} & \multicolumn{3}{|c|}{ Sales for Resale } & \multicolumn{3}{|c|}{$\begin{array}{l}\text { Exchange } \\
\text { Dellvered }\end{array}$} & \multicolumn{3}{|c|}{$\begin{array}{l}\text { Wheeling } \\
\text { Dellvered }\end{array}$} \\
\hline & $1989^{2}$ & 1988 & 1987 & $1989^{7}$ & 1988 & 1987 & $1989^{2}$ & 1988 & 1987 & $1989^{\prime \prime}$ & 1988 & 1987 \\
\hline ECAH & 245,874 & 232.759 & 203,923 & 152.703 & 159,063 & 134,897 & 72.177 & 58,661 & 58.745 & 20,994 & 15,036 & 10,281 \\
\hline EACOOT & 76,668 & 75,182 & 72,681 & 32,355 & 31.905 & 30,085 & 35,017 & 35,364 & 36,053 & 9,297 & 7,913 & 6.543 \\
\hline MAAC & 55,290 & 47,506 & 48,345 & 17.940 & 16,628 & 16,602 & 28.990 & 23,513 & 24,518 & 4,360 & 7.465 & 7,226 \\
\hline MAIN & 60,612 & 56,863 & $50,5,34$ & 23,746 & 21,291 & 23,395 & 35,661 & 33,666 & 25,123 & 1,205 & 1.906 & 2,017 \\
\hline MAPP(U.S.) $\ldots \ldots \ldots$ & 105,500 & 108.391 & 107.771 & 59,451 & 62,928 & $69,520^{\circ}$ & 37,852 & 38,757 & 31,524 & 8.197 & 6,705 & 6.727 \\
\hline NPCCQUS.) $\ldots \ldots \ldots \ldots \ldots \ldots$ & 164,638 & 154,736 & 135,192 & 99.653 & 97,267 & 99,725 & 22,634 & 19,380 & 15,456 & 42,351 & 38,089 & 20.011 \\
\hline SERC $\ldots \ldots \ldots$ & 375,248 & 330,155 & 335.384 & 277,693 & 261,562 & 265,950 & 75,670 & 56,850 & 59,468 & 21,645 & 11,743 & 9,966 \\
\hline SPP & 134,684 & 125.734 & 117,047 & 96,202 & 94,034 & 88,488 & 18,558 & 11,743 & 18,741 & 19,924 & 19,956 & 9,818 \\
\hline WSCC(U.S.) & 469,146 & 428,207 & 388,896 & 213,689 & 199,053 & 185,867 & 91,833 & 71,472 & 84,034 & 163,623 & 157,682 & 118,995 \\
\hline Conitiguous U.S. & $1,687,660$ & $1,559,633$ & $1,459,774$ & 973,432 & 943,731 & 914,528 & 418,392 & 349,406 & 353,661 & 295,836 & 266,496 & 191,584 \\
\hline Alaska & 2,705 & 2,590 & 2,543 & 2,244 & 2,177 & 2,096 & $\bullet$ & 0 & 1 & 461 & 413 & 446 \\
\hline Hawail" & 3 & 89 & 83 & 0 & 85 & 79 & 3 & 4 & 4 & 0 & 0 & 0 \\
\hline U.S. Total & $1,690,368$ & $1,562,312$ & $1,462,400$ & 975,676 & 945,993 & 916,703 & 418,395 & 349,410 & 353,667 & 296,297 & 266,909 & 192,030 \\
\hline
\end{tabular}

- Equals sales for resalo plus exchange delivored plus wheeling delivered

- Data lor 1989 are preliminary.

Hawail includes nonutility transactions only

Value less than 0.5

Notes: - Totals may not equal sum of components because of independent rounding. - Summation of utility trade lor utilities that operate within the NEHC Region. Due to the complexity of electric power transactioris that involve specifics of contracts, simultaneous energy transactions, the unintended receip: and deliver: of energy (inadvertent flow), and loss('s, uniformity in reporting the classification and quantity of each transaction among utilities may not exist. Includes ut lity, import, export, and nonutility iransactions.

Source: Ene gy Iniormation, Administration, Form EIA-861, "Annual Electric Utility Fieport." 
Table 55. Utility Net Energy Flow by North American Electric Reliability Council Region, Alaska, and Hawail, 1987-1989

(Million Killowatthours)

\begin{tabular}{|c|c|c|c|c|c|c|c|c|c|}
\hline \multirow{2}{*}{$\begin{array}{l}\text { North American Electric } \\
\text { Rellabllity Counc'l Region, } \\
\text { Alaska, and Hawall }\end{array}$} & \multicolumn{3}{|c|}{$\begin{array}{c}\text { Net } \\
\text { Energy Flow' }\end{array}$} & \multicolumn{3}{|c|}{ Recelpts ${ }^{2}$} & \multicolumn{3}{|c|}{ Dellveries } \\
\hline & $1989^{4}$ & 1888 & 1987 & $1989^{4}$ & 1088 & 1987 & $1888^{4}$ & 1988 & 1987 \\
\hline ............... & $-39,874$ & $-46,647$ & $-42,824$ & 206,000 & 186,112 & 161,099 & 245,874 & 232,759 & 203,923 \\
\hline ERCOT & 25,833 & 26,460 & 24,043 & 102,501 & 101,642 & 96,724 & 76,668 & 75,182 & 72,681 \\
\hline MAAC & 30,977 & 28,780 & 22,528 & 86,267 & 76,386 & 70,874 & 55,290 & 47,606 & 48,345 \\
\hline MAIN & 3,178 & 1,894 & 6,141 & 63,790 & 58,756 & 56,675 & 60,612 & 56,863 & 50,534 \\
\hline MAPP(U.S.) & 8,746 & 6,363 & $-2,562$ & 114,246 & 114,754 & 105,209 & 105,500 & 108,391 & 107,771 \\
\hline NPCC(UIS.) & 19,361 & 23,927 & 24,344 & 183,999 & 178,664 & 159,536 & 164,638 & 154.736 & 135,192 \\
\hline SERC & 8,416 & 18,369 & 4,141 & 383,664 & 348,524 & 339,525 & 375,248 & 330,155 & 335,384 \\
\hline SPP & 5,552 & 4,582 & -918 & 140,237 & 130,316 & 116,128 & 134,684 & 125,734 & 117,047 \\
\hline WSCC(U.S.) ........ & 16.726 & 15,138 & 16,161 & 485,872 & 443,345 & 405,057 & 469,146 & 428,207 & 388,896 \\
\hline Contiguous U.S. & 78,915 & 78,866 & 51,054 & $1,766,575$ & $1,638,499$ & $1,510,828$ & $1,687,660$ & $1,558,633$ & $1,459,774$ \\
\hline Alaska & 41 & 17 & -7 & 2,746 & 2,607 & 2,536 & 2,705 & 2,590 & 2,543 \\
\hline Hawail .... & 449 & 408 & 398 & 452 & 497 & 481 & 3 & 89 & 83 \\
\hline U.S. Total ........ & 79,405 & 79,292 & 51,445 & $1,769,773$ & $1,641,604$ & $1,513,845$ & $1,690,368$ & $1,562,312$ & $1,462,400$ \\
\hline
\end{tabular}

1 Equals receipts minus deilveries

Equals purchased power plus exchange received plus wheeling received.

- Equals sales for resale plus exchange delivered plus wheeling delivered.

- Data for 1989 are preliminary.

- Hawaii includes nonutility transactions only.

Notes: - Totals may not equal sum of components because of independent rounding. - Summalion of utility trade for. utilties that operate within the NERC Region. Due to the complexity of electric power transactions that involve specifics of contracts, simultaneous energy transactions, the unintended receipt and delivery of onergy (inadvertent llow), and losses, uniformity in reporting the classification and quantity of each transaction among utilities may not exist. Includas ututity, import, export, and nonutility transactions.

Source. Energy Infoimation Administration, Form EIA.861. "Annual Electric Utility Report."

Table 56. Utility Purchases of Nonutility Generated Electricity by North American Electric Reliability Council Region, Alaska, and Hawail, 1987-1989

(Million Kilowatthours)

\begin{tabular}{|c|c|c|c|}
\hline $\begin{array}{l}\text { Norih American Electric Rellabillty } \\
\text { Councll Region, Alaska, and Hawall }\end{array}$ & $1989^{\prime}$ & 1988 & 1987 \\
\hline ............................... & 2,024 & 1,214 & 606 \\
\hline ................................ & 22,694 & 22,170 & 21,976 \\
\hline MAAC & 5,479 & 2.550 & 871 \\
\hline MAIN & 179 & 103 & 32 \\
\hline MAPP(US.) & 148 & 97 & 73 \\
\hline NPCC(U.S.) & 10,182 & 6,316 & 4,205 \\
\hline SERC & 8.914 & 8.103 & 4,365 \\
\hline SPP & 2,811 & 1,319 & 997 \\
\hline WSCC(US) & 37,145 & 25,939 & 16,787 \\
\hline Contiguous U.S. & 89,576 & 67,810 & 49,812 \\
\hline Alaska & 1 & 0 & 47 \\
\hline 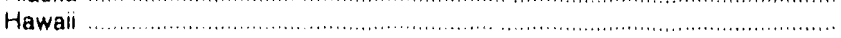 & 2.35 & 220 & 206 \\
\hline U.S. Tolal & 89,813 & 68,030 & 50,165 \\
\hline
\end{tabular}

- Data for 1989 are preliminary

- Value less than 0.5 million kilowatthours.

Note: Totals may not equal sum of components because of independent rounding.

Source: Energy Intormation Administration, Form ElA-861, "Annual Electric Utility Report." 
Table 57. Net Imports by North American Electric Rellability Council Region, Alaska, and Hawail, 1987-1989

(Thousand Kilowatthours)

\begin{tabular}{|c|c|c|c|}
\hline $\begin{array}{l}\text { North American Electrlc Rellabillty Councll Reglon, } \\
\text { Alaska, and Hawail }\end{array}$ & 1989 & 1988 & 1987 \\
\hline ECAR & $-5,426,212$ & 164,437 & 770,056 \\
\hline ERCOT & $-10,315$ & $-11,762$ & $-11,355$ \\
\hline MAAC & NA & NA & NA \\
\hline MAIN & NA & NA & NA \\
\hline MAPP(U.S.) & $-373,343$ & $-1,279,546$ & $3,325,019$ \\
\hline NPCC(U.S.) & $11,147,862$ & $23,349,907$ & $28,023,192$ \\
\hline SERC & NA & NA & NA \\
\hline SPP & NA & NA & NA \\
\hline WSCC(U.S.) & $5,637,053$ & $9,546,828$ & $14,230,947$ \\
\hline Contiguous U.S. & $10,975,843$ & $31,769,864$ & $46,337,859$ \\
\hline Alaska & 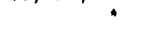 & 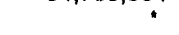 & * \\
\hline 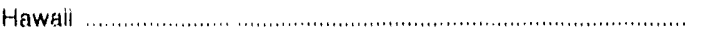 & NA & NA & NA \\
\hline U.S. Total & $10,975,843$ & $31,769,864$ & $46,337,859$ \\
\hline
\end{tabular}

- Value less than 0.5

NA Not applicable.

Notes: - Totals may not equal sum of components because of independent rounding. . Values identify point of entry or exit, but do not necessarily identify point of consumption. - These data reflect electricity imported from Canada and Mexico. -Net imports data represent gross imports minus gross exports.

Source. Oflice of Fuals Progiams, Fossil Energy, Form FE-781月, "Annual Report of International Electric Import/Export Data."

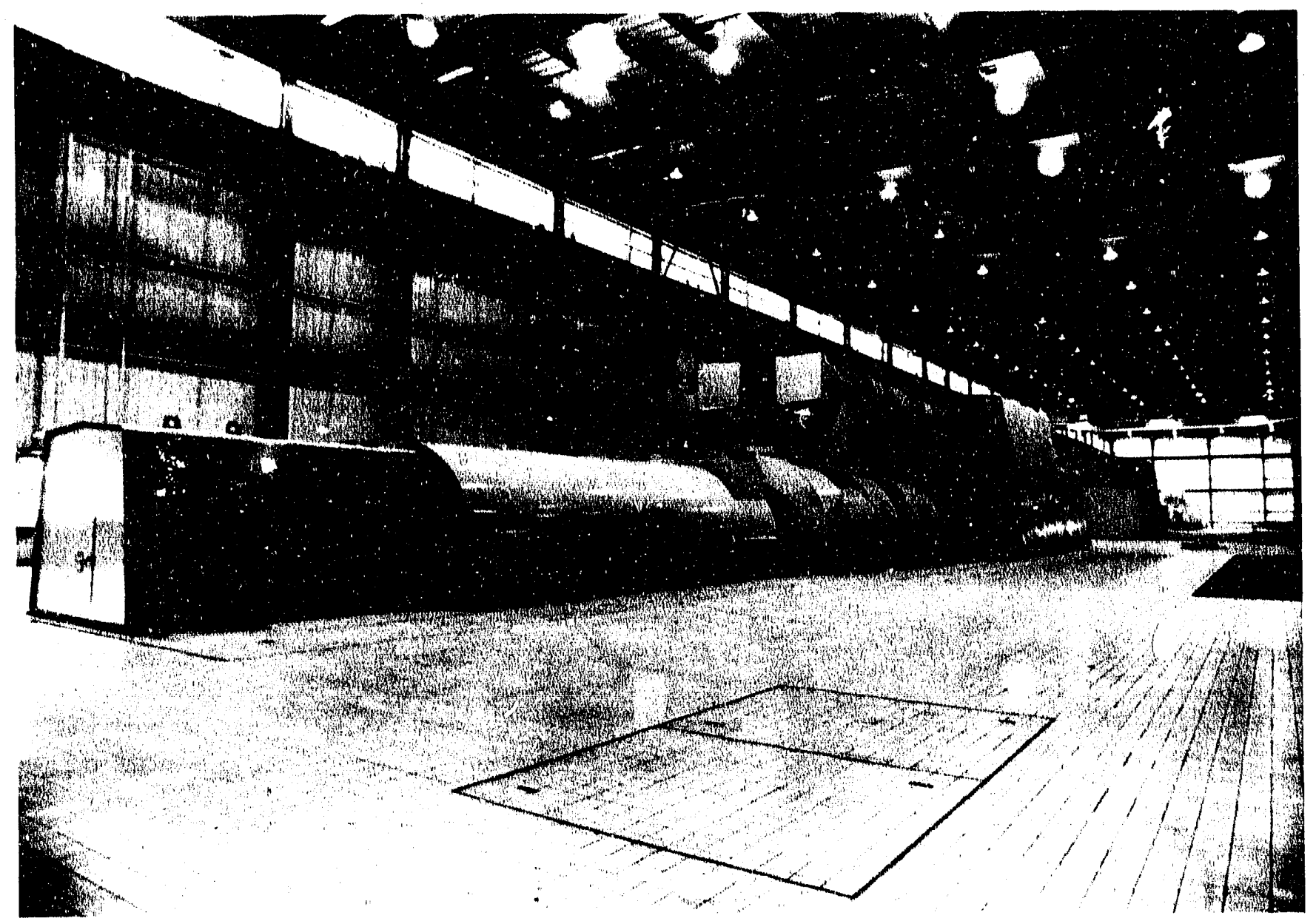

Operation and maintenance expenses consist of maintaining the physical condition and operating efficiency of the plant, including the generation room shown here. 
Appendix A

Major

Disturbances and

Unusual

Occurrences 


\section{Appendix A}

\section{Major Disturbances and Unusual Occurrences}

Electric power systems are subject to a variety of incidents that, to a smaller or greater degree, may adversely affect the delivery of electricity to consumers. Among these are natural phenomena (such as storms and earthquakes); failure of electric system components; accidental or purposeful activities inimical to continued safe operation of electric power systems; and difficulties associated with the normal operation of large, extremely complex, real-time systems.

Under current Federal regulations, some disturbances are reported to the Federal Government. The legal basis for the requirements and the specifications of information reported are detailed in Title 10, Part 205, Subpart W, of the Code of Federal Regulations, Sections 205.350 through 205.353, published in the Federal Register, October 31, 1986. Underlying the formulation of the reporting criteria, requirements and procedures was the need for the Federal Government to be aware of potentially dangerous situations, tempered by the desire to minimize burden on the reporting utilities. Another consideration in the development of the rules was the benefit gained from knowledge of the causes and effects of undesired events that may have been caused by unforeseen systcm defects or by purposeful adverse actions to system design and operation. The final rules reflect modification of the preliminary rules, as published in the Federal Register, based on comments from the electric power industry and the general public.

In general, the incidents to be reported are grouped into two categories: mandatory in all cases; and mandatory if the incident meets specified criteria. A report is mandatory in all cases when, for the purpose of maintaining the reliability of the bulk power supply system, a utility (1) initiates a system voltage reduction of 3 percent or more, (2) disconnects circuits supplying over 100 megawatts of firm customer load, or (3) issues an appeal to the public for a voluntary reduction in the use of electricity. A report is also mandatory in regard to any actual or suspected act of sabotage or terrorism directed at the bulk power supply system. For the second category of mandatory incidents, the electric utility involved is permitted to exercise some judgment as to whether the criteria requiring mandatory reporting have been met.

In general, reports are to be made by telephone to the Emergency Operating Center, Department of Energy, in Washington, DC, as soon as practicable for instances of load shedding or loss of service, and, at the latest, within 3 hours of the beginning of a service interruption. For other disturbances, the allowable reporting time ranges from 24 hours to days. Written reports may be required by the Director, Office of Energy Emergency Operations, if the circumstances so indicate.

The operation of the bulk power system in the United States should be as trouble-free as possible. To that end, information is collected, as discussed above, regarding major disturbances to the normal functioning of that system. Events, such as damage to some local distribution circuits by storms or other uncontrollable events, do not greatly affect the supply of bulk power to the system as a whole. These events are more properly the concern of local and State authorities.

\section{Data Sources}

The information contained in Table $\mathrm{Al}$ is based on data from the Form IE-417R, "Electric Power System Emergency Report." These data are collected by the Office of Energy Emergency Operations (under the Assistant Secretary for International Affairs and Energy Emergencies). 
Table A1. Major Disturbances and Unusual Occurrences, 1989

\begin{tabular}{|c|c|c|c|c|c|c|c|}
\hline Date & Time & Area & $\begin{array}{l}\text { Utility/Power Pool } \\
\text { (NERC Council) }\end{array}$ & $\begin{array}{c}\text { Type of } \\
\text { Disturbance }\end{array}$ & $\begin{array}{c}\text { Loss } \\
\text { (megawatt) }\end{array}$ & $\begin{array}{l}\text { Number of } \\
\text { Customers } \\
\text { Affected }\end{array}$ & $\begin{array}{c}\text { Restoration } \\
\text { Time }\end{array}$ \\
\hline $3 / 13 / 89$ & $2: 45$ AM & $\begin{array}{l}\text { Quebec, } \\
\text { Canada }\end{array}$ & $\begin{array}{l}\text { Hydro-Quebec } \\
\text { (NPCC) }\end{array}$ & $\begin{array}{l}\text { 'Solar } \\
\text { Storm }\end{array}$ & 21,000 & NA & $\begin{array}{c}\text { Noon } \\
\text { Same Day }\end{array}$ \\
\hline $6 / 01 / 89$ & 5:06 PM & $\begin{array}{l}\text { Maryland, } \\
\text { Washington, DC }\end{array}$ & $\begin{array}{l}\text { Potomac Electric } \\
\text { Power Company } \\
\text { (PJM) }\end{array}$ & Int. & 305 & 47,500 & $\begin{array}{l}\text { 7:23 PM } \\
\text { Same Day }\end{array}$ \\
\hline $6 / 02 / 89$ & 1:00 PM & Virginia & $\begin{array}{l}\text { Virginia Power } \\
\text { Company } \\
\text { (SERC) }\end{array}$ & ${ }^{\prime} 5 \%$ VR & 300 & $1,650,000$ & $\begin{array}{l}\text { 8:30 PM } \\
\text { Same Day }\end{array}$ \\
\hline $6 / 05 / 80$ & $12: 30$ AM & $\begin{array}{l}\text { Washington, } \\
\text { DC }\end{array}$ & $\begin{array}{l}\text { Potomac Electric } \\
\text { Power Company } \\
\text { (PJM) }\end{array}$ & “Cable fire & 35 & 500 & $\begin{array}{l}\text { 8:00 AM } \\
\text { Next Day }\end{array}$ \\
\hline $6 / 14 / 89$ & 3:00 PM & $\begin{array}{l}\text { Birmingham, } \\
\text { Alabama }\end{array}$ & $\begin{array}{l}\text { Alabama Power } \\
\text { Company } \\
\text { (SERC) }\end{array}$ & 'Storms & 200 & 51,000 & $\begin{array}{l}10: 00 \text { PM } \\
\text { Next Day }\end{array}$ \\
\hline $6 / 14 / 89$ & 3:00 PM & $\begin{array}{l}\text { Washington, } \\
\text { DC }\end{array}$ & $\begin{array}{l}\text { Potomac Electric } \\
\text { Power Company } \\
\text { (PJM) }\end{array}$ & 'Storm & 700 & 150,000 & $6 / 16 / 89$ \\
\hline $6 / 17 / 89$ & $10: 00 \mathrm{PM}$ & $\begin{array}{l}\text { Omaha, } \\
\text { Nebraska }\end{array}$ & $\begin{array}{l}\text { Omaha Public } \\
\text { Power District } \\
\text { (MAPP) }\end{array}$ & 'Int & 4 & NA & $\begin{array}{l}\text { 4:00 AM } \\
\text { Next Day }\end{array}$ \\
\hline $6 / 22 / 89$ & 3:54 PM & $\begin{array}{l}\text { Sarnta Clara, } \\
\text { Cailfornia }\end{array}$ & $\begin{array}{l}\text { Santa Clara } \\
\text { Electric Department } \\
\text { (WSCC) }\end{array}$ & Int & 247 & 8,200 & $\begin{array}{l}\text { 5:39 PM } \\
\text { Same Day }\end{array}$ \\
\hline $6 / 25 / 89$ & 5:27 PM & Hawaii & $\begin{array}{l}\text { Hawaii Electric } \\
\text { Light Company }\end{array}$ & Int & NA & NA & $\begin{array}{l}\text { 6:03 PM } \\
\text { Same Day }\end{array}$ \\
\hline $6 / 27 / 89$ & 1:44 PM & $\begin{array}{l}\text { Western } \\
\text { Maryland }\end{array}$ & $\begin{array}{l}\text { Potomac Edison } \\
\text { Company } \\
\text { (ECAR) }\end{array}$ & IovR & 30 & NA & $\begin{array}{l}\text { 6:00 PM } \\
\text { Same Day }\end{array}$ \\
\hline $6 / 27 / 89$ & 1:28 PM & $\begin{array}{l}\text { PjM } \\
\text { Service } \\
\text { Area }\end{array}$ & $\begin{array}{l}\text { PJM } \\
\text { Interconnection } \\
\text { (MAAC) }\end{array}$ & "VR & 900 & NA & $\begin{array}{c}\text { 6:00 PM } \\
\text { Same Day }\end{array}$ \\
\hline $6 / 27 / 89$ & 1:30 PM & $\begin{array}{l}\text { Virginia Power } \\
\text { Company } \\
\text { Service Area }\end{array}$ & $\begin{array}{l}\text { Virginia Power } \\
\text { Company } \\
\text { (SERC) }\end{array}$ & "VR & 300 & $1,700,000$ & $\begin{array}{l}\text { 6:00 FM } \\
\text { Same Day }\end{array}$ \\
\hline $7 / 01 / 89$ & 3:38 PM & $\begin{array}{l}\text { Kansas City, } \\
\text { Kansas }\end{array}$ & $\begin{array}{l}\text { Kansas City Power } \\
\text { and Light Company } \\
\text { (SPP) }\end{array}$ & "Lightning & 200 & About 510 & $\begin{array}{c}\text { 6:15 PM } \\
\text { CDT } \\
\text { Same Day }\end{array}$ \\
\hline $7 / 10 / 89$ & 4:15 PM & $\begin{array}{l}\text { Connecticut, } \\
\text { Massachusetts }\end{array}$ & $\begin{array}{l}\text { New England } \\
\text { Power Pool } \\
\text { (NPCC) }\end{array}$ & ${ }^{14}$ Storms & 100 & 95,000 & $7 / 14 / 89$ \\
\hline $7 / 14 / 89$ & 2:34 PM & South Carolina & $\begin{array}{l}\text { South Carolina Electric } \\
\text { and Gas Company } \\
\text { (SERC) }\end{array}$ & $\begin{array}{l}\text { "System } \\
\text { Problem }\end{array}$ & NA & 200,000 & $\begin{array}{l}\text { 4:30 PM } \\
\text { Same Day }\end{array}$ \\
\hline $7 / 14 / 89$ & 2:04 PM & $\begin{array}{l}\text { Pennsylvania, } \\
\text { Maryland, } \\
\text { Virginia }\end{array}$ & $\begin{array}{l}\text { PJM } \\
\text { Interconnection } \\
\text { (MAAC) }\end{array}$ & "VA & 168 & $1,270,000$ & $\begin{array}{l}\text { 7:25 PM } \\
\text { Same Day }\end{array}$ \\
\hline $7 / 14 / 89$ & NA & $\begin{array}{l}\text { Jim Bridger } \\
\text { Power Plant, } \\
\text { Wyoming }\end{array}$ & $\begin{array}{l}\text { Pacific Power } \\
\text { and Light Company } \\
\text { (WSCC) }\end{array}$ & "Fire & None & None & $\begin{array}{l}\text { Estimate } \\
\text { One month }\end{array}$ \\
\hline $7 / 18 / 89$ & NA & $\begin{array}{l}\text { Near } \\
\text { Joliet, } \\
\text { Itlinois }\end{array}$ & $\begin{array}{l}\text { Conimonwealth Edison } \\
\text { Company } \\
\text { (MAIN) }\end{array}$ & "Lightning & None & None & NA \\
\hline $7 / 24 / 89$ & $7: 22 \mathrm{AM}$ & $\begin{array}{l}\text { Near } \\
\text { Elkhorn City, }\end{array}$ & $\begin{array}{l}\text { Appalachian Power } \\
\text { Company }\end{array}$ & "Sabotage & None & None & $8 / 7 / 89$ \\
\hline
\end{tabular}

See endnotes at the end of the table. 
Table A1. Major Disturbances and Unusual Occurrences, 1989-Continued

\begin{tabular}{|c|c|c|c|c|c|c|c|}
\hline Date & Time & Area & $\begin{array}{c}\text { Utility/Power Pool } \\
\text { (NERC Council) }\end{array}$ & $\begin{array}{c}\text { Type of } \\
\text { Disturbance }\end{array}$ & Loss & $\begin{array}{l}\text { Number of } \\
\text { Customers } \\
\text { Affected }\end{array}$ & $\begin{array}{l}\text { Restoration } \\
\text { Tlime }\end{array}$ \\
\hline & & Kentucky & (ECAR) & & & & \\
\hline $7 / 26 / 89$ & $1: 54 \mathrm{PM}$ & $\begin{array}{l}\text { PJM } \\
\text { Area } \\
\text { Service }\end{array}$ & $\begin{array}{l}\text { PJM } \\
\text { Interconnection } \\
\text { (MAAC) }\end{array}$ & $\begin{array}{l}{ }^{20} \text { High Load } \\
\text { and VR }\end{array}$ & NA & NA & $\begin{array}{l}\text { 6:00 PM } \\
\text { Same Day }\end{array}$ \\
\hline $7 / 26 / 89$ & $2: 45 \mathrm{PM}$ & $\begin{array}{l}\text { Long island, } \\
\text { New York }\end{array}$ & $\begin{array}{l}\text { Long Island Lighting } \\
\text { Company } \\
\text { (NPCC) }\end{array}$ & "High Load & NA & NA & $\begin{array}{l}\text { 8:00 PM } \\
\text { Same Day }\end{array}$ \\
\hline $8 / 04 / 89$ & $9: 58$ AM & $\begin{array}{l}\text { Glendale, } \\
\text { California }\end{array}$ & $\begin{array}{l}\text { Glendale Public Service } \\
\text { Department } \\
\text { (WSCC) }\end{array}$ & $\begin{array}{l}{ }^{22} \text { Tie Line } \\
\text { Trip }\end{array}$ & 148 & 74,000 & $\begin{array}{l}\text { Noon } \\
\text { Same Day }\end{array}$ \\
\hline $8 / 14 / 89$ & $1: 26 \mathrm{PM}$ & $\begin{array}{l}\text { Glendale, } \\
\text { California }\end{array}$ & $\begin{array}{l}\text { Glendale Public Service } \\
\text { Departiment } \\
\text { (WSCC) }\end{array}$ & $\begin{array}{l}{ }^{23} \text { Disconnect } \\
\text { Switch }\end{array}$ & 63 & NA & $\begin{array}{l}\text { 3:46 PM } \\
\text { Same Day }\end{array}$ \\
\hline $8 / 14 / 89$ & $2: 51 \mathrm{PM}$ & $\begin{array}{l}\text { Lake Worth, } \\
\text { Florida }\end{array}$ & $\begin{array}{l}\text { City of Lake Worth } \\
\text { Utilities } \\
\text { (SERC) }\end{array}$ & ${ }^{24}$ Lightning & 57 & 26,000 & $\begin{array}{l}\text { 3:40 PM EDT } \\
\text { Same Day }\end{array}$ \\
\hline $8 / 16 / 89$ & $1: 30 \mathrm{PM}$ & $\begin{array}{l}\text { Canoga Park. } \\
\text { California }\end{array}$ & NA & ${ }^{25}$ Accident & NA & Test Facility & $\begin{array}{l}\text { 12:30 PM } \\
\text { Next Day }\end{array}$ \\
\hline $8 / 20 / 89$ & $4: 11 P M$ & $\begin{array}{l}\text { Central } \\
\text { Florida }\end{array}$ & $\begin{array}{l}\text { Florida Power Corporation } \\
\text { and other systems } \\
\text { (SERC) }\end{array}$ & ${ }^{26} \mathrm{Int}$ & 2,970 & 132,000 & $\begin{array}{l}\text { 5:17 PM } \\
\text { Same Day }\end{array}$ \\
\hline $9 / 11 / 89$ & $1: C 0 P M$ & $\begin{array}{l}\text { Long Island, } \\
\text { New York }\end{array}$ & $\begin{array}{l}\text { Long Island Lighting } \\
\text { Company } \\
\text { (NPCC) }\end{array}$ & $\begin{array}{l}{ }^{2 v} \text { High Load } \\
\text { and VR }\end{array}$ & NA & NA & $\begin{array}{l}\text { 8:00 PM } \\
\text { Same Day }\end{array}$ \\
\hline $9 / 18 / 89$ & Early AM & $\begin{array}{l}\text { St. Croix, } \\
\text { Virgin Islands }\end{array}$ & $\begin{array}{l}\text { Virgin Islands Water } \\
\text { and Power Authority }\end{array}$ & $\begin{array}{l}\text { 28Hurricane } \\
\text { Hugo }\end{array}$ & NA & NA & $\begin{array}{l}\text { Estimated- } \\
\text { year-end }\end{array}$ \\
\hline $9 / 18 / 89$ & Early AM & $\begin{array}{l}\text { St. Johns, } \\
\text { Virgin Islands }\end{array}$ & $\begin{array}{l}\text { Virgin Islands Water } \\
\text { and Power Authority }\end{array}$ & $\begin{array}{l}\text { "Hurricane } \\
\text { Hugo }\end{array}$ & NA & NA & $\begin{array}{l}\text { October- } \\
\text { November }\end{array}$ \\
\hline $9 / 18 / 89$ & Early AM & $\begin{array}{l}\text { St.Thomas, } \\
\text { Virgin Islands }\end{array}$ & $\begin{array}{l}\text { Virgin Islands Water } \\
\text { and Power Authority }\end{array}$ & $\begin{array}{l}\text { Hurricane } \\
\text { Hugo }\end{array}$ & NA & NA & $\begin{array}{l}70 \% \text { by } \\
\text { Oct } 23\end{array}$ \\
\hline $9 / 19 / 89$ & Mid-day & Puerto Rico & $\begin{array}{l}\text { Puerto Rico Electric } \\
\text { Power Authority }\end{array}$ & $\begin{array}{l}{ }^{20} \text { Hurricane } \\
\text { Hugo }\end{array}$ & NA & NA & $\begin{array}{l}\text { Most by late } \\
\text { September }\end{array}$ \\
\hline $9 / 22 / 89$ & $A M$ & $\begin{array}{l}\text { South Carolina, } \\
\text { North Caroiina }\end{array}$ & $\begin{array}{l}\text { Duke Power Company } \\
\text { (SERC) }\end{array}$ & $\begin{array}{l}\text { 2aHurricane } \\
\text { Hugo }\end{array}$ & NA & 696,348 & $10 / 8 / 89$ \\
\hline $9 / 22 / 89$ & $8: 00 \mathrm{AM}$ & South Carolina & $\begin{array}{l}\text { Carolina Power and Light } \\
\text { Company } \\
\text { (SERC) }\end{array}$ & $\begin{array}{l}\text { 20 Hurricane } \\
\text { Hugo }\end{array}$ & NA & 140,000 & $10 / 9 / 89$ \\
\hline $9 / 22 / 89$ & $10: 00 \mathrm{AM}$ & $\begin{array}{l}\text { Virginia } \\
\text { (S.W. Area) }\end{array}$ & $\begin{array}{l}\text { Appalachian Power Company } \\
\text { (ECAR) }\end{array}$ & $\begin{array}{l}\text { "Huiricane } \\
\text { Hugo }\end{array}$ & NA & 92,127 & $9 / 24 / 89$ \\
\hline $9 / 22 / 89$ & 10:00 AM & $\begin{array}{l}\text { West Virginia } \\
\text { (S.E. Area) }\end{array}$ & $\begin{array}{l}\text { Appalachian Power Company } \\
\text { (ECAR) }\end{array}$ & $\begin{array}{l}\text { "Hurricant } \\
\text { ! yugo }\end{array}$ & NA & 39,053 & $9 / 24 / 89$ \\
\hline $9 / 22 / 89$ & $A M$ & South Carolina & $\begin{array}{l}\text { South Carolina Electric } \\
\text { and Gas Company } \\
\text { (SERC) }\end{array}$ & $\begin{array}{l}{ }^{20} \text { Hurricane } \\
\text { Hugo }\end{array}$ & NA & 300,000 & NA \\
\hline $9 / 22 / 89$ & NA & Pennsylvania & $\begin{array}{l}\text { West Penn Power Company } \\
\text { (ECAR) }\end{array}$ & $\begin{array}{l}\text { Hurricane } \\
\text { Hugo }\end{array}$ & NA & 16,000 & $9 / 23 / 89$ \\
\hline
\end{tabular}

See endnotes at the end of the table. 
Table A1. Major Disturbances and Unusual Occurrences, 1989-Continued

\begin{tabular}{|c|c|c|c|c|c|c|c|}
\hline Date & Time & Area & $\begin{array}{l}\text { Utility/Power Pool } \\
\text { (NERC Council) }\end{array}$ & $\begin{array}{l}\text { Type of } \\
\text { Disturbance }\end{array}$ & $\begin{array}{c}\text { Loss } \\
\text { (megawatt) }\end{array}$ & $\begin{array}{l}\text { Number of } \\
\text { Customers } \\
\text { Affected }\end{array}$ & $\begin{array}{c}\text { Restoration } \\
\text { Time }\end{array}$ \\
\hline $9 / 22 / 89$ & PM & $\begin{array}{l}\text { Long Island, } \\
\text { New York }\end{array}$ & $\begin{array}{l}\text { Long Island Lighting } \\
\text { Company } \\
\text { (NPCC) }\end{array}$ & $\begin{array}{l}\text { "Hurricane } \\
\text { Hugo }\end{array}$ & NA & 109,000 & $\begin{array}{l}3: 00 \mathrm{PM} \\
9 / 23 / 89\end{array}$ \\
\hline $9 / 22 / 89$ & $A M$ & $\begin{array}{l}\text { Central and } \\
\text { Western Maryland }\end{array}$ & $\begin{array}{l}\text { Potomac Edison Company } \\
\text { (ECAR) }\end{array}$ & $\begin{array}{l}{ }^{2 a} \text { Hurricane } \\
\text { Hugo }\end{array}$ & NA & 6,000 & $\begin{array}{l}\text { Evening } \\
\text { Same Day }\end{array}$ \\
\hline $9 / 22 / 89$ & $1: 00 P M$ & West Virginia & $\begin{array}{l}\text { Monongahela Power Company } \\
\text { (ECAR) }\end{array}$ & $\begin{array}{l}\text { 2ayurricane } \\
\text { Hugo }\end{array}$ & NA & 17,000 & $\begin{array}{l}\text { Noon } \\
9 / 24 / 90\end{array}$ \\
\hline $9 / 22 / 8 \theta$ & $A M$ & South Carolina & $\begin{array}{l}\text { South Caroiina Public } \\
\text { Service Authority } \\
\text { (SERC) }\end{array}$ & $\begin{array}{l}\text { "Hurricane } \\
\text { Hugo }\end{array}$ & NA & NA & NA \\
\hline $10 / 02 / 89$ & $11: 30 \mathrm{AM}$ & $\begin{array}{l}\text { Nassau County, } \\
\text { Now York }\end{array}$ & $\begin{array}{l}\text { Long Island Lighting } \\
\text { Company } \\
\text { (NPCC) }\end{array}$ & ${ }^{2}$ Cable Cut & NA & NA & $\begin{array}{l}\text { 2:00 PM } \\
\text { Next Day }\end{array}$ \\
\hline $10 / 14 / 89$ & 11:00 PM & $\begin{array}{l}\text { Galveston Bay } \\
\text { Area of Texas }\end{array}$ & $\begin{array}{l}\text { Houston Lighting and } \\
\text { Power Company } \\
\text { (ERCOT) }\end{array}$ & $\begin{array}{l}{ }^{30} \text { Hurricane } \\
\text { Jerry }\end{array}$ & 300 & 75,000 & $\begin{array}{l}\text { Morning } \\
10 / 15 / 89\end{array}$ \\
\hline $10 / 17 / 89$ & $\begin{array}{l}\text { 5:04 PM } \\
\text { PST }\end{array}$ & $\begin{array}{l}\text { Loma Prieta, } \\
\text { California }\end{array}$ & $\begin{array}{l}\text { Pacific Gas \& Electric } \\
\text { Company } \\
\text { (WSCC) }\end{array}$ & ${ }^{31}$ Earthquake & 2,000 & 491,000 & $\begin{array}{l}6: 00 \text { AM } \\
10 / 23 / 89\end{array}$ \\
\hline $10 / 17 / 89$ & 5:04 PM & $\begin{array}{l}\text { Santa Clara, } \\
\text { California }\end{array}$ & $\begin{array}{l}\text { Santa Clara Electric } \\
\text { Depaltment } \\
\text { (WSCC) }\end{array}$ & ${ }^{31}$ Earthquake & 240 & 32,915 & $\begin{array}{l}10 / 19 / 89 \\
\text { End of Day }\end{array}$ \\
\hline $10 / 17 / 89$ & 5:04 PM & $\begin{array}{l}\text { Alameda, } \\
\text { California }\end{array}$ & $\begin{array}{l}\text { Alameda Bureau of } \\
\text { Electricity } \\
\text { (WSCC) }\end{array}$ & "Earthquake & 35 & 9,670 & $\begin{array}{l}\text { Within } \\
2 \text { hours }\end{array}$ \\
\hline $10 / 17 / 89$ & 5:04 PM & $\begin{array}{l}\text { Modesto, } \\
\text { California }\end{array}$ & $\begin{array}{l}\text { Modesto trrigation } \\
\text { District } \\
\text { (WSCC) }\end{array}$ & ${ }^{3}$ Earthquake & 291 & NA & NA \\
\hline $10 / 17 / 89$ & 5:04 PM & $\begin{array}{l}\text { Palo Alto, } \\
\text { California }\end{array}$ & $\begin{array}{l}\text { Palo Alto Electric } \\
\text { Utility } \\
\text { (WSCC) }\end{array}$ & "Earthquake & 160 & 21,600 & $10 / 20 / 89$ \\
\hline $10 / 17 / 89$ & 5:04 PM & $\begin{array}{l}\text { Turlock, } \\
\text { California }\end{array}$ & $\begin{array}{l}\text { Turlock Irrigation } \\
\text { Districl } \\
\text { (WSCC) }\end{array}$ & ${ }^{31}$ Earthquake & 45 & 9,800 & $10 / 19 / 89$ \\
\hline $10 / 19 / 89$ & $1: 30 \mathrm{AM}$ & $\begin{array}{l}\text { Cincinnati, } \\
\text { Ohio }\end{array}$ & $\begin{array}{l}\text { Cincinnati Gas and } \\
\text { Electric Company } \\
\text { (ECAR) }\end{array}$ & $\begin{array}{l}{ }^{32} \text { Winter lce } \\
\text { Storm }\end{array}$ & 600 & 100,000 & NA \\
\hline $11 / 08 / 89$ & $\begin{array}{l}3: 59 \text { PM } \\
\text { MST }\end{array}$ & WSCC Area & $\begin{array}{l}\text { Several systems in } \\
\text { WSCC }\end{array}$ & $\begin{array}{l}{ }^{33} \text { Trans } \\
\text { Line Fault }\end{array}$ & 260 & NA & $\begin{array}{l}\text { 5:00 PM } \\
\text { Same Day }\end{array}$ \\
\hline $11 / 16 / 89$ & $6: 00 \mathrm{AM}$ & $\begin{array}{l}\text { Huntsville and } \\
\text { Northern } \\
\text { Alabama }\end{array}$ & $\begin{array}{l}\text { Tennessee Valley } \\
\text { Authority (TVA) } \\
\text { (SERC) }\end{array}$ & $\begin{array}{l}{ }^{34} \text { Severe } \\
\text { Weather }\end{array}$ & 1,000 & 60,000 & Several Days \\
\hline $11 / 20 / 89$ & $11: 30 \mathrm{PM}$ & $\begin{array}{l}\text { Washington, } \\
\text { D.C. Area }\end{array}$ & $\begin{array}{l}\text { Potomac Electric } \\
\text { Power Company } \\
\text { (MAAC) }\end{array}$ & ${ }^{35}$ High Wirnds & NA & 20,000 & $\begin{array}{l}\text { Noon } \\
\text { Next Day }\end{array}$ \\
\hline $11 / 20 / 89$ & 9:00 PM & $\begin{array}{l}\text { Philadelphia, } \\
\text { Pennsylvania } \\
\text { Area }\end{array}$ & $\begin{array}{l}\text { Philadelphia } \\
\text { Electric Company } \\
\text { (MAAC) }\end{array}$ & ${ }^{38} \mathrm{High}$ Winds & NA & 90,000 & $\begin{array}{l}\text { Noon } \\
\text { Next Day }\end{array}$ \\
\hline $11 / 20 / 89$ & 4:00 PM & Long Island, & Long Island Lighting & ${ }^{3 b} \mathrm{High}$ Winds & NA & 54,000 & Noon \\
\hline
\end{tabular}

See endnotes at the end of the table. 
Table A1. Major Disturbances and Unusual Occurrences, 1989-Continued

\begin{tabular}{|c|c|c|c|c|c|c|c|}
\hline Date & Time & Area & $\begin{array}{c}\text { Utility/Power Pool } \\
\text { (NERC Council) }\end{array}$ & $\begin{array}{c}\text { Type of } \\
\text { Disturbance }\end{array}$ & $\begin{array}{c}\text { Loss } \\
\text { (megawatt) }\end{array}$ & $\begin{array}{l}\text { Number of } \\
\text { Customers } \\
\text { Affected }\end{array}$ & $\begin{array}{l}\text { Restoration } \\
\text { Time }\end{array}$ \\
\hline & & New York & $\begin{array}{l}\text { Company } \\
\text { (NPCC) }\end{array}$ & & & & Next Day \\
\hline $11 / 20 / 89$ & 9:20 PM & $\begin{array}{l}\text { Westchester, } \\
\text { County. } \\
\text { New York }\end{array}$ & $\begin{array}{l}\text { Consolidated Edison } \\
\text { of New York } \\
\text { (NPCC) }\end{array}$ & ${ }^{36} \mathrm{High}$ Winds & NA & 17,800 & $\begin{array}{r}9: 00 \text { AM } \\
\text { Next Day }\end{array}$ \\
\hline $12 / 04 / 89$ & 7:00 AM & New England & $\begin{array}{l}\text { Now England } \\
\text { Power Pool } \\
\text { (NPCC) }\end{array}$ & $\begin{array}{l}{ }^{36} \text { High Loads } \\
\text { and } 5 \% \\
\text { VR }\end{array}$ & NA & NA & $\begin{array}{l}\text { 8:00 PM } \\
\text { Same Day }\end{array}$ \\
\hline $12 / 04 / 89$ & 4:58 PM & $\begin{array}{l}\text { Long Isiand, } \\
\text { New York }\end{array}$ & $\begin{array}{l}\text { Long Island Lighting } \\
\text { Company } \\
\text { (NPCC) }\end{array}$ & ${ }^{37} \mathrm{VR}$ & NA & NA & $\begin{array}{l}\text { 7:01 PM } \\
\text { Same Day }\end{array}$ \\
\hline $12 / 04 / 89$ & 4:58 PM & $\begin{array}{l}\text { Consolidated } \\
\text { Edison Area of } \\
\text { New York }\end{array}$ & $\begin{array}{l}\text { Consolidated Edison } \\
\text { Company of New York } \\
\text { (NPCC) }\end{array}$ & $\begin{array}{l}\text { "Load } \\
\text { Curtailed }\end{array}$ & NA & NA & $\begin{array}{l}\text { 6:02 PM } \\
\text { Same Day }\end{array}$ \\
\hline $12 / 05 / 89$ & 5:00 PM & $\begin{array}{l}\text { New York } \\
\text { State }\end{array}$ & $\begin{array}{l}\text { New York } \\
\text { Power Pool } \\
\text { (NPCC) }\end{array}$ & ${ }^{34} 5 \%$ VA & NA & NA & $\begin{array}{l}\text { 5:52 PM } \\
\text { Same Day }\end{array}$ \\
\hline $12 / 06 / 89$ & NA & $\begin{array}{l}\text { Syracuse, } \\
\text { New York }\end{array}$ & $\begin{array}{l}\text { Niagara Mohawk } \\
\text { Power Corporation } \\
\text { (NPCC) }\end{array}$ & $\begin{array}{l}{ }^{* 0} \text { Int } \\
\text { and VR }\end{array}$ & NA & NA & $\begin{array}{l}\text { 7:00 PM } \\
\text { Same Day }\end{array}$ \\
\hline $12 / 08 / 89$ & 2:50 PM & $\begin{array}{l}\text { Consolidated } \\
\text { Edison Area of } \\
\text { New York }\end{array}$ & $\begin{array}{l}\text { Consolidated Edison } \\
\text { Company of New York } \\
\text { (NPCC) }\end{array}$ & $\begin{array}{l}\text { "Load } \\
\text { Curtailed }\end{array}$ & NA & NA & $\begin{array}{l}\text { 5:24 PM } \\
\text { Same Day }\end{array}$ \\
\hline $12 / 13 / 89$ & 5:00 PM & $\begin{array}{l}\text { New York } \\
\text { State }\end{array}$ & $\begin{array}{l}\text { New York } \\
\text { Power Pool } \\
\text { (NPCC) }\end{array}$ & ${ }^{42} 5 \%$ VR & 50 & 300,000 & $\begin{array}{l}\text { 6:00 PM } \\
\text { Same Day }\end{array}$ \\
\hline $12 / 14 / 89$ & 6:00 PM & New England & $\begin{array}{l}\text { New England } \\
\text { Power Pool } \\
\text { (NEPOOL) }\end{array}$ & $\begin{array}{l}{ }^{43} \mathrm{VA} \\
\text { and PA }\end{array}$ & 240 & $1,000,000$ & $\begin{array}{l}\text { 8:00 PM } \\
\text { Same Day }\end{array}$ \\
\hline $12 / 16 / 89$ & $A M$ & $\begin{array}{l}\text { Homer, } \\
\text { Alaska }\end{array}$ & $\begin{array}{l}\text { Homer Electric } \\
\text { Association }\end{array}$ & ${ }^{44}$ Volcanic & NA & 13,800 & $\begin{array}{l}\text { 9:00 PM } \\
\text { Same Day }\end{array}$ \\
\hline $12 / 22 / 89$ & 7:35 AM & $\begin{array}{l}\text { Washington, DC, } \\
\text { Baltimore, MD }\end{array}$ & $\begin{array}{l}\text { Potomac Electric } \\
\text { Power Company } \\
\text { Baltimore Gas and Elec (PJM) }\end{array}$ & $\begin{array}{l}{ }^{45} \text { High Loads } \\
\text { and VF }\end{array}$ & NA & NA & $\begin{array}{l}\text { 11:34 PM } \\
\text { Same Day }\end{array}$ \\
\hline $12 / 22 / 89$ & $9: 23$ AM & $\begin{array}{l}\text { Houston, } \\
\text { Texas }\end{array}$ & $\begin{array}{l}\text { Houston Lighting and } \\
\text { Power Company } \\
\text { (ERCOT) }\end{array}$ & ${ }^{46} 2.5 \%$ VA & NA & NA & $\begin{array}{l}\text { 9:59 AM } \\
\text { Same Day }\end{array}$ \\
\hline $12 / 22 / 89$ & $10: 00 A . M$ & $\begin{array}{l}\text { Houston, } \\
\text { Texas }\end{array}$ & $\begin{array}{l}\text { Houston Lighting and } \\
\text { Power Company } \\
\text { (ERCOT) }\end{array}$ & ${ }^{40} 5 \%$ VR & 250 & NA & $\begin{array}{l}11: 00 P M \\
12 / 23 / 89\end{array}$ \\
\hline $12 / 22 / 89$ & NA & $\begin{array}{l}\text { Austin, } \\
\text { Texas }\end{array}$ & $\begin{array}{l}\text { Lower Colorado } \\
\text { River Authority }\end{array}$ & $\begin{array}{l}\text { "High Loads } \\
\text { and PA }\end{array}$ & NA & NA & $\begin{array}{l}11: 00 P M \\
12 / 23 / 89\end{array}$ \\
\hline $12 / 22 / 89$ & $10: 30 \mathrm{AM}$ & $\begin{array}{l}\text { Abilene, } \\
\text { Texas }\end{array}$ & $\begin{array}{l}\text { West Texas } \\
\text { Utilities Company }\end{array}$ & "PA & NA & NA & $\begin{array}{l}\text { Noon } \\
\text { Same Day }\end{array}$ \\
\hline $12 / 23 / 89$ & $7: 40 \mathrm{AM}$ & $\begin{array}{l}\text { Abilene, } \\
\text { Texas }\end{array}$ & $\begin{array}{l}\text { West Texas } \\
\text { Utilities Company }\end{array}$ & "PA & NA & NA & $\begin{array}{l}\text { 12:40 PM } \\
\text { Same Day }\end{array}$ \\
\hline $12 / 23 / 89$ & 6:00 PM & $\begin{array}{l}\text { St. Petersburg. } \\
\text { Florida }\end{array}$ & $\begin{array}{l}\text { Florida Power } \\
\text { Corporation } \\
\text { (SERC) }\end{array}$ & "VR & 400 & 61,000 & $\begin{array}{c}\text { 8:00 PM } \\
\text { Same Day }\end{array}$ \\
\hline $12 / 23 / 89$ & $7: 15$ AM & Corpus Christi, & Central Power & ${ }^{50} \mathrm{PA}$ & 55.5 & NA & 10:37 AM \\
\hline
\end{tabular}


Table A1. Major Disturbances and Unusual Occurrences, 1989-Continued

\begin{tabular}{|c|c|c|c|c|c|c|c|}
\hline Date & Time & Area & $\begin{array}{l}\text { Utillty/Power Pool } \\
\text { (NERC Council) }\end{array}$ & $\begin{array}{c}\text { Type of } \\
\text { Disturbance }\end{array}$ & $\underset{\text { (megawatt) }}{\text { Loss }}$ & $\begin{array}{c}\text { Number of } \\
\text { Customers } \\
\text { Affected }\end{array}$ & $\begin{array}{c}\text { Restoration } \\
\text { Time }\end{array}$ \\
\hline & & Texas & and Light Company & & & & Same Day \\
\hline $12 / 23 / 89$ & $12: 14$ AM & $\begin{array}{l}\text { Jacksonville, } \\
\text { Florida }\end{array}$ & $\begin{array}{l}\text { Jacksonville Electric } \\
\text { Authority }\end{array}$ & "'PA & 211 & 50,000 & $\begin{array}{l}\text { 12:44 PM } \\
\text { Same Day }\end{array}$ \\
\hline $12 / 23 / 8 \theta$ & NA & $\begin{array}{l}\text { Orlando, } \\
\text { Florida }\end{array}$ & $\begin{array}{l}\text { Orlando Utilities } \\
\text { Commission }\end{array}$ & "High Loads & NA & $\begin{array}{l}4 \% \text { of } \\
\text { system }\end{array}$ & $\begin{array}{r}12 / 23 / 89 \\
\text { Same Day }\end{array}$ \\
\hline $12 / 24 / 8 \theta$ & 8:50 AM & $\begin{array}{l}\text { St. Petersburg, } \\
\text { Florida }\end{array}$ & $\begin{array}{l}\text { Florida Power } \\
\text { Corporation } \\
\text { (SERC) }\end{array}$ & $\begin{array}{l}\text { "VR } \\
\text { and PA }\end{array}$ & 1,200 & 247,000 & $\begin{array}{c}\text { 1:00 PM } \\
\text { Same Day }\end{array}$ \\
\hline $12 / 24 / 89$ & 6:56 AM & $\begin{array}{l}\text { Jacksonville, } \\
\text { Florida }\end{array}$ & $\begin{array}{l}\text { Jacksonville Electric } \\
\text { Authority }\end{array}$ & ${ }^{B} 5 \%$ VR & 45 & 290,000 & $\begin{array}{r}\text { 9:28 AM } \\
\text { Same Day }\end{array}$ \\
\hline $12 / 24 / 89$ & 10:03 AM & $\begin{array}{l}\text { Jacksonville, } \\
\text { Florida }\end{array}$ & $\begin{array}{l}\text { Jacksonville Electric } \\
\text { Authority }\end{array}$ & $.15 \%$ VA & 22 & 145,000 & $\begin{array}{l}\text { 10:30 AM } \\
\text { Same Day }\end{array}$ \\
\hline $12 / 24 / 89$ & $6: 00 \mathrm{AM}$ & $\begin{array}{l}\text { Lake Worth, } \\
\text { Florida }\end{array}$ & $\begin{array}{l}\text { Lake Worth } \\
\text { Municipal Utility }\end{array}$ & $\begin{array}{l}{ }^{{ }_{3} 5} \text { steps } \\
\text { each } \\
1.5 \% \text { VR }\end{array}$ & 12 & NA & $\begin{array}{l}\text { 11:00 AM } \\
\text { Same Day }\end{array}$ \\
\hline $12 / 24 / 89$ & NA & $\begin{array}{l}\text { Tampa, } \\
\text { Florida }\end{array}$ & $\begin{array}{l}\text { Tampa Electric } \\
\text { Company }\end{array}$ & "S4R & NA & NA & $12 / 25 / 89$ \\
\hline $12 / 24 / 89$ & $6: 08 \mathrm{AM}$ & $\begin{array}{l}\text { Miami, } \\
\text { Florida }\end{array}$ & $\begin{array}{l}\text { Florida Power and } \\
\text { Light Company } \\
\text { (SERC) }\end{array}$ & ${ }^{86} \mathrm{VP}$ & $\begin{array}{l}100 \text { to } \\
2,800\end{array}$ & $\begin{array}{l}16 \% \text { of } \\
\text { system }\end{array}$ & $\begin{array}{l}\text { 11:30 AM } \\
\text { Same Day }\end{array}$ \\
\hline $12 / 24 / 89$ & $6: 09 \mathrm{AM}$ & $\begin{array}{l}\text { Mlami, } \\
\text { Florida }\end{array}$ & $\begin{array}{l}\text { Florida Power } \\
\text { and Llght Company } \\
\text { (SERC) }\end{array}$ & "SR & $\begin{array}{l}100 \text { to } \\
2,800\end{array}$ & $\begin{array}{l}16 \% \text { of } \\
\text { system }\end{array}$ & $\begin{array}{c}\text { 6:15 PM } \\
\text { Same Day }\end{array}$ \\
\hline $12 / 24 / 89$ & $8: 18 \mathrm{AM}$ & $\begin{array}{l}\text { Miami, } \\
\text { Florida }\end{array}$ & $\begin{array}{l}\text { Florida Power } \\
\text { and Light Company } \\
\text { (SERC) }\end{array}$ & ${ }^{B 6}$ VR & $\begin{array}{l}100 \text { to } \\
2,800\end{array}$ & $\begin{array}{l}16 \% \text { of } \\
\text { system }\end{array}$ & $\begin{array}{r}\text { 10:17 AM } \\
\text { Samv Day }\end{array}$ \\
\hline $12 / 25 / 89$ & $8: 09$ AM & $\begin{array}{l}\text { St. Petersburgh, } \\
\text { Florida }\end{array}$ & $\begin{array}{l}\text { Florida Power } \\
\text { Corporation } \\
\text { (SERC) }\end{array}$ & $\begin{array}{l}\text { "VP } \\
\text { and PA }\end{array}$ & 815 & 156,000 & $\begin{array}{l}\text { 9:46 AM } \\
\text { Same Day }\end{array}$ \\
\hline $12 / 25 / 89$ & $6: 00 \mathrm{AM}$ & $\begin{array}{l}\text { Lake Worth, } \\
\text { Florida }\end{array}$ & $\begin{array}{l}\text { Lake Worth } \\
\text { Municipal Utility }\end{array}$ & $\begin{array}{l}{ }^{35} \text { steps } \\
\text { each } \\
1.5 \% \text { VR }\end{array}$ & 11 & NA & $\begin{array}{l}11: 00 \text { AM } \\
\text { Same Day }\end{array}$ \\
\hline $12 / 25 / 89$ & 4:57 AM & $\begin{array}{l}\text { Miami, } \\
\text { Florida }\end{array}$ & $\begin{array}{l}\text { Florida Power } \\
\text { and Light Company } \\
\text { (SERC) }\end{array}$ & sVR & $\begin{array}{l}100 \text { to } \\
2,800\end{array}$ & $\begin{array}{l}16 \% \text { of } \\
\text { system }\end{array}$ & $\begin{array}{l}11: 14 \text { AM } \\
\text { Same Day }\end{array}$ \\
\hline $12 / 25 / 89$ & 2:00 PM & $\begin{array}{l}\text { Kissimmee, } \\
\text { Floride }\end{array}$ & $\begin{array}{l}\text { Kissirnmee Utility } \\
\text { Authority }\end{array}$ & $\begin{array}{l}{ }^{\text {"OCircuit }} \\
\text { Breaker } \\
\text { Tripped }\end{array}$ & 190 & 31,000 & $\begin{array}{c}\text { 3:30 PM } \\
\text { Same Day }\end{array}$ \\
\hline $12 / 26 / 89$ & 6:00 AM & $\begin{array}{l}\text { Lake Wnrth, } \\
\text { Florida }\end{array}$ & $\begin{array}{l}\text { Lake Worth } \\
\text { Municipal Utility }\end{array}$ & $\begin{array}{l}\text { 2 steps } \\
\text { each } \\
1.5 \% \text { VR }\end{array}$ & 4 & NA & $\begin{array}{l}\text { 11:00 AM } \\
\text { Same Day }\end{array}$ \\
\hline $12 / 27 / 89$ & NA & $\begin{array}{l}\text { Syracuse, } \\
\text { New York }\end{array}$ & $\begin{array}{l}\text { Niagara Mohawk } \\
\text { Power } \\
\text { Corporation }\end{array}$ & ${ }^{87} \mathrm{PA}$ & NA & NA & NA \\
\hline $12 / 29 / 89$ & NA & $\begin{array}{l}\text { Consolidated } \\
\text { Edison Area of } \\
\text { New York }\end{array}$ & $\begin{array}{l}\text { Consolidated Edison } \\
\text { of New York } \\
\text { (NPCC) }\end{array}$ & ${ }^{*}$ Fire & 180 & 137,070 & $\begin{array}{l}\text { 5:05 PM } \\
\text { Same Day }\end{array}$ \\
\hline $12 / 30 / 89$ & NA & $\begin{array}{l}\text { Consolidated } \\
\text { Edison Area of } \\
\text { New York }\end{array}$ & $\begin{array}{l}\text { Consolidated Edison } \\
\text { of New York } \\
\text { (NPCC) }\end{array}$ & "Fire & NA & NA & NA \\
\hline
\end{tabular}

See endnotes at the end of the table. 
Table A1. Major Disturbances and Unusual Occurrences, 1989-Continued

\begin{tabular}{|c|c|c|c|c|c|c|c|}
\hline Date & Time & Area & $\begin{array}{l}\text { Utillty/Power Pool } \\
\text { (NERC Councll) }\end{array}$ & $\begin{array}{l}\text { Type of } \\
\text { Disfurbance }\end{array}$ & $\begin{array}{c}\text { Loss } \\
\text { (megawatt) }\end{array}$ & $\begin{array}{l}\text { Number of } \\
\text { Customers } \\
\text { Affected }\end{array}$ & $\begin{array}{l}\text { Restoration } \\
\text { Time }\end{array}$ \\
\hline $12 / 21 / 89$ & NA & $\begin{array}{l}\text { Bangor, } \\
\text { Maine }\end{array}$ & $\begin{array}{l}\text { Bangor } \\
\text { Hydroelectric } \\
\text { Company }\end{array}$ & $\begin{array}{l}\text { "2:Switch } \\
\text { Tripped }\end{array}$ & NA & 90,000 & Same Day \\
\hline
\end{tabular}

' A severe solar system storm caused shutdown of the Hydro-Quebec system, cutting off sales of power by Hydro-Quebec to New England and New York. There were no disruptions of service in New York or in New England and both of those areas were able to supply power to assist HydroQuebec. Voltage fluctuations, unusual noise in transformers, and some tripping of capacitor banks occurred in the WSCC area, apparently caused by solar magnetic effects but not related to the situation in Quebec.

2 Faiture of a secondary bushing on a 69-kilovolt circuit breaker resulted in the destruction of that breaker, a fire, and damage to other facilities in the Bells Mill substation. Five other substations were shut down by protectlve relay equipment as a result of the damage to Bells Mill substation.

3 Voltage reduction necessitated by unseasonal high temperatures causing unusually high loads, 3 nuclear units out of service for maintenance: and refueling, 2 large fossil units out for maintenance, 2 smaller units out for maintenance, inability to import desired purchase capacity because of low voltage on seller's (APS) transmission system, forced outage of Possum Point No. 5 (a large fossil unit belonging to PEPCO)

1 Fire in a manhole on M Street N.W. damaged 13.2-kilovolt underground cables and spread to other manholes. Commercial and government of. fice buildings in central D.C. lost all power and some 15,000 employees were affected. Federal agencies without electricity were: Federal Communications Commission; the Washington Office of the U.S. Secret Service; Administrative Council of the United States; Railroad Retirement Board. Traffic signals in the area were inoperable and several streets were closed by police.

s A severe thunderstorm and high winds damaged distribution and subtransmission circuits.

- The most severe storm in PEPCO history downed about 500 distribution lines, knocked down poles and trees and required massive reconstruction.

, During work in an underground transformer vault a short circuit occurred on the 480 -volt secondary of a 13.8-kilovolt/480-volt transformation. Three men were injured (one died the next day from burns) and service to downtown Omaha was interrupted.

- Damage to a transformer at Kefer Receiving Station; cause not known.

- An earthquake near the Island of Hawail caused several distribution circuits to trip. There was no damage to generating piants or transmission facilities.

10 Loss of a Chalk Point unit on the Potomac Electric Power Company system caused power flow and voltage level problems that forced Potomac Edison Company to reduce its entire system voltage by 5 percent in order maintain system integrity.

"As a result of the loss of a Chalk Point unit on the syster. of Potomac Electric Power Co. (PEPCO) the entire PJM system had to reduce voltage by 5 percent. The Potomac Edison Company also had to reduce voltage by 5 percent.

12 A 5-percent voltage reduction was needed as a result of PEPCO's loss of unit at Chalk Point.

13 Lightning struck one of the 161-kilovolt transmission lines on the low side of the 345-kilovolt/161-kilovolt transformation at Hawthorn Substation. The line circuit breaker failed to trip and the backup relaying also failed to operate. Other circuit breakers in the substation tripped, opening the 345. kilovolt and 161 kilovolt busses. Some equipment sustained minor damage.

14 Thunderstorms caused numerous distribution circuit outages.

is System operating problems caused voltage fluctuations and loss of several generating units including V.C. Summer Nuclear Unit.

is Lack of sufficient operable generating capacity to meet increasing load necessitated a 5-percent voltage reduction by Potomac Electric Power Company and the Baltimore Gas and Electric Company members of the PJM Power Pool. In order to prevent break-up of the interconnected system the Allegheny Power System (ECAR) and Virginia Power Company (SERC) also reduced voltage by 5 percent.

17 Electrical failure on the low-voltage side of the 560 MVA generator transformer for one of the four 500-megawatt generating units at the plant (near Rock Springs, Wyoming) caused a fire that damaged the transformer and overhead conductors leading to the switchyard. There was no loss of service.

1" During an electrical storm the reactor containment buildings of Braidwood Nuclear Units 1 and 2 were struck by lightning. The two strikes, about 6 minutes apart, caused overvoltage relays on the reactor control rods to operate and shut down the units. There was no damage to the plant.

10 One tower of a 765-kilovolt transmission line fell because it was damaged by explosives.

${ }^{20}$ Heavy loads and inability to obtain purchased power from systems to the west (ECAR area) made a 5-percent voltage reduction necessary.

21 Heavy loads required Long Island Lighting Company (LILCO) to institute Stage 2 of their Power System Emergency Alert Procedure: reduce LILCO in-house use of power; request some 500 large industrial customers and 16 transmission-line customers to reduce or interrupt their loads; im. plement direct load control of residential air conditioners, water heaters and swimming pool pumps; issue a radio appeal to the public to reduce the use of power.

22 City of Glendale has it own generators supplemented by power from the Los Angeles Department of Water and Power. One line was out of service and the second line failed. The load exceeded the capacity of Glendale's own generators and the system failed.

${ }^{23}$ Failıre of a disconnect switch on a distribution circuit in the central business district caused loss of power on the circuit.

24 lightning. No details available.

25 Power Supply to 9 offices and 3 test facilities of the Department of Energy 's ETEC Test Facility at the Santa Susanna Field Laboratory in San Francisco was interrupted when a contractor, installing a groundwater monitoring well, inadvertently drilled through a 4,160-volt cable. Power to the offices was restored at 3:30 PM on the same day. Power to the Test Facility was restored on August 17.

20 The 500-kilovolt interconnections between Florida Power Corporation and the Southern Company (Georgia) tripped. Frequency declined to 59.37 hertz, causing automatic shedding of load by under-frequency relays. Sixteen utilities shed a total of 2,970 megawatts including 1,576 megawatts in the service area of Florida Power and Light Company, 500 megawatts on the Florida Power Corporation system, 275 megawatts on Jacksonville Electric Authority's system and 260 megawatts on the system of Tampa Electric Company. The incident was caused by failure of a 500-kilovolt switch at Georgia Power Company's Plant Hatch and a resultant phase-to-phase short circuit on the Duval (Florida) to Hatch (Georgia) 500-kilovolt transmission line. At the time of the fault the Southern Company (including Georgia Power) was supplying 3,136 megawatts to the Florida systems.

27 High loads, loss of Nine Mile Point 2 (LILCO share 179 megawatts) and some forced and scheduled generator outages required LILCO to institute load control measures at 1:00 PM. Later loss of 3 gas turbines forced implementation of a 5-percent voltage reduction at 4:48 PM. Voltage was restored to normal at 6:15 PM and the load control measures were cancelled at 8:00 PM.

${ }_{20}$ Hurricane Hugo struck the Virgin Islands during the early morning of September 18, moved on to Puerto Rico on the 19th and hit the coas of South Carolina on the 22nd. It then moved inland in a northwesterly direction, turned north and then veered eastward. Most of the hurricane's force was spent by the time it left the Carolinas. Because a large number of systems were involved it is impracticable to list in detail all the damage that was done. Many transmission line towers and structures were knocked down by the force of the winds. As towers and poles fell they broke conductors, unbalancing the stress of unbroken lines on other structures and making thern more susceptible to the winds. The same is true of distribution lines, which suffered additionally from the effects of broken trees and flying debris. No generating plants were reported as damaged

20 An underground cable to Nassau Community College was inadvertently cut by a bulldozer during construction work on an office building project.

Several U.S. Navy buildings near the College, and some residences, also lost power.

* Hurricane Jerry caused an interruption of power supply.

${ }^{31}$ An earthquake struck the San Francisco area (epicenter near Loma Prieta), damaging Pacific Gas and Electric Company electric and gas facill- 
ties and interrupting power to approximately 565,000 customers on a number of systems. On the Pacific Gas and Electric system the bulk power supply facility that sutfered the most damage was the Moss Landing Plant, where the switchyard was partially destroyed; at Metcalf subsiation all the 500 . kilovolt circuit breakers were destroyed. At several PG\&E plants generating units tripped as the electrical shock was transmitted through the system and transmission lines opened. Distribution circults were destroyed in some areas, particularly the Marina area of San Francisco and in Santa Cruz.

Systems other than PG\&E do not appear to have suffered damage to electrical facilities to any extent; loss of customer service seems to have re. sulted from electrical disturbances to the interconnected system caused by loss of PG\&E lines and load. The sudden loss of a large part of the PG\&E load caused high voltage in some parts of the interconnected system and some frequency fluctuations. Tripping of transmission lines and loss of some generators on the PG\&E system, combined with the physical shock of the earthquake, caused protective relays to operate and trip some loads on surrounding systems.

32 Ice storm caused distribution circuits to fail.

33 A short circuit of one phase to ground occurred on the Broadview-Garrison No. 1500 -kilovolt line and was closely followed by a similar fault on the No. 2 line. As a result of the consequent abnormal flows of current on the interconnected system several lines and generating units tripped in Montana, Colorado, New Mexico. Utah and Wyoming. Low frequency was experienced hy Southern California Edison Company and the PacificCorp Electric System East; South Dakota and Idaho were also affected.

34 A severe weather front including tornadoes destroyed a 46 -kilovolt/12-kilovolt substation and a number of transmission line structures at the 161 . kilovolt level. A public appeal for conservation of electricity was issued.

${ }_{35}$ A severe windstorm over the mid-Atlantic states caused numerous interruptions of customer service on several systems. Five $69-k i l o v o l t s ~ t r a n s-$ mission lines on the LILCO system were temporarily put out of service by the winds, as were lines on the PEPCO system at voltage levels of 69 kilovolts, 34 kilovolts and 13 kilovolts. No damage to generating plants was reported by any system. In addition to the customer outages listed in the table, other outages (but no details) were reported as follows: Baltimore Cias and Electric Company 70,000; Pennsylvania Power and Light Company 113,000; Altantic City Electric Company 5,000; Delmarva Power and Light Company 8,000.

36 The system experienced a deficiency of generating capacity due to unexpected high loads, cutback of purchased power deliveries from New York and a number of unplanned outages of NEPOOL generating units. Public appeals for power conservation were issued, a 5-percent voltage reduction was put into effect and various other load reduction measures were employed. The actual peak load served was 19,082 megwatts. It is estimate that the load would have been 19,800 megawatts if all the load reduction measures had not been used. There was no actual disconnection of customers. The difficulties began about 7:00 AM and were essentially over by about 8:00 PM.

${ }^{17}$ Large bulk power customers served directly from transmission lines were requested to curtail their loads due to shortage of reserve generating capacity on the New York Power Pool System. The request was issued 4:58 PM and was rescinded at 7:01 PM.

3. Curtailment of some loads was instituted at 4:58 PM in cooperation with Long Island Lighting Company and other member systems of the New York Power Pool. The restriction was lifted a 6:02 PM

30 A pool-wide voltage reduction of 5 percent was ordered at 5:00 PM and was cancelled at 5:52 PM.

40 The company was requested by the New York Power Pool to institute load reduction measures because the Pool was short of reserve generating capacity. The company took the following measures: interrupted customers served under contracts for interruptible service; curtailed the company's own use of power; asked large customers to voluntarily cut their use of power. No estimate of number of customers affected or magnitude of power reduction achieved is available. The emergency was terminated at about 7:00 PM.

1 At the request of the New York Pool, New York which expected a shortage of reserve capacity during the afternoon, the company requested its major consumers to curtail their loads at 2:50 PM. The system was restored to normal at 5:24 PM.

42 Fool members having facilities for manual voltage reduction were requested to implement a 5-percent reduction such that adequate operating reserve wnuld be available for the peak load period from 5:00 PM to 6:00 PM. About 300,000 customers were affected and load was reduced by about 50 megawatts.

${ }^{43}$ Manual and automatic voltage reductions and a public appeal were initiated at 6:00 PM, affecting about 1 million customers and resulting in a load reduction of about 2.40 megawatts. The actions were cancelled at 8:00 PM. Cold weather caused high loads and unexpected forced outages caused reserve margins to decline below the minimum set by standards for reliable service.

4A About 3,800 customers were affected by outages lasting up to 8 hours and some 10,000 customers suffered momentary outages all due to volcanic ash from an eruption of Mt. Redoubt settling on 14,400-volt lighting arrestors, cutouts and other apparatus, causing flashovers. The disturbance began in the morning on December 16 and continued until about 9:00 PM

${ }^{65}$ Both companies instituted a 5-percent voltage reduction between 7:35 AM and 11:34 AM be :ause cold weather caused unexpected high loads and problems in delivery of No. 6 fuel oil required for system operation, such that oil would be contierved.

${ }_{40}$ Unusually widespread severe cold weather caused unexpected load increases and difficulty in operation at some power plants with outdoor facilities not designed for operation under such weather conditions. In addition the cold weather increased residential use of natural gas and reduced the gas available to power plants. A 2.5-percent voltage reduction was instituted at 9:23 AM and was superseded by a 5-percent reduction at 10:00 AM. The 5-percent reduction in voltage is estimated to to have reduced load by about 250 megawatts.

47 Unusually cold weather caused unexpected load increases. A public appeal was issued for conservation of electricity.

4a Extreme culd weather caused unusually high demand and a variety of mishaps reduced the generating capacity that was available. A public appeal for load conseivation was made on December 22 at 10:30 AM and was cancelled at 12:00 noon. There was no interruption of service to customers. On December 23 a public appeal to reduce load was made at 7:40 AM and was rescinded at 12:40 PM.

40 Due to unexpected cold weather, loads were very high. A public appeal to conserve electricity was issued, voltage reduction and load manage ment programs were implemented, interruptible customers were interrupted, curtailable customers were curtailed and some firm load was interrupted via rotating blackouts. The rotating blackouts involved 61,000 customers and 400 megawatts of load on December 23 (6.00 PM to 8:00 PM; 247,000 customers and 1,200 megawatts of load on December 24 8:50 AM to 1:00 PM); 156,000 customers and 815 megawatts of load on December 25 (8:09 AM to $9: 46$ AM).

so A radio appeal was made for reduction of load at 7:15 AM and the appeal was carried on TV at 9:00 F V. The company shed 35 megawatts of firm load about 10:20 AM and by 10:27 AM this had increased to about 55.5 megawatts. By 10:37 AM the load that had been shed was restored. The company's use of natural gas was restricted and some loss of generating capacity was experienced. The situation was due to abnormally cold weather over most of the state of Texas. The public appeal was cancelled on the morning of December 24

51 Extreme cold weather caused difficulties in the operation of the Authority's system due to high demano, tie-line overloads and the forced outage of two 600 megawatt generating units. A public appeal to reduce loads was issued on December 23 at $12: 14$ PM and was rescinded at 12:44 PM on the same day; about 50,000 customers and some 211 megawatis of load were affected. On December 24 a 5 -percent voltage reduction was instifuted at 6:56 AM and was cancelled at 9:28 AM, affecting about 290,000 customers and reducing load by about 45 megawatts. A 5-percent voltage reduction was again in effect to December 24 from 10:03 AM to 10:30 AM, affecting 145,000 customers and reducing load by about 22 megawatts.

52 Extremely cold weather caused high loads. High demands and loss of some generation necessitated a voltage reduction of 2.5 percent and 4 percent of the system's customers were shed from some 10 minutes.

53 Cold weather caused unusual demand on the system. Automatic voltage reduction, in 5 steps of 1.5 percent each, was implemented, a 30 -year old generating plant in cold stand-by was re-activated and the Municipal Water and Sewer Department was asked to operate its emergency generator. About 12 megawatts load was lost due to the voltage reduction.

st Load reductions and curtailments due to unusually cold weather and increased electrical demand for heating

ss Extremely low temperatures beginning on the morning of December 24 , caused Florida Power and Light to experience energy deficiencies due to a combination of high loads, generating unit maintenance outages, gas supply shortages and forced outages of generating units. Rotating feeder interruptions were instituted. During the 2-day problern period there were public appeals to reduce use of electric power. The maximum number of customers without service at one time was about 480,000 (some $16 \%$ of the load). Altogether, about 40 percent of the system 's customers experienced outages during the rotating blackouts.

so Same situation occurred as on December 24. This time load reduction was about 11 megawatts.

5) A 59-kilovolt breaker tripped. system ties to other utilities were overloaded, and the Kissimmee system then separated from the Florida Interconnected System.

st Same stivation as December 24 and 25 . This tirne only 2 voltage reduction steps (1.5 percent each) were implemented. The loss reduction was 
estimated to have been 4 megawatts.

so New York Pool declared the region to be in "Reserve Condition No. 1". Utilities were to have appeal to customers tor curtailed use of electric-

ity.

so Fire in the Hell Gate switching station caused cutoff of electric power to the Yorkville and Bronx service areas of the company. The fire was believed to have been caused by rupture of a natural gas line that passed through or near the switching station, which :ras formerly a generating plant.

61 Power supply to an area in the Bronx was out for an hour due to a gas line fire.

92 Complete outage due to a switch station falling on a 70-MVA transformer. Some 90,000 customers lost servicie for an hour.

Note: The rules promulgated in the Code of Federal Regulations (10 CFR Part 205) and published in the Federal Register of October 31 , 1986, do not require utilities to report distribution system outages that are local in nature and affect only a small area or a small number of customers. Utilities may report such outages if they so desire:

EDT $=$ Eastern Daylight Time,

EST :-: Eastern Standard Time,

Int $=$ Interruption

$L F=$ Line Fault (Accidental contact of energized conductors or of energized conductors with ground)

$L R=$ Load Reduction,

LTNG =: Lightning Strike,

MAST = Mountain Advanced Standard Time,

MW:- Megawatts,

NA = Not Available.

PA:: Public Appeal,

PJM - Pennsylvania, New Jersey, Maryland.

UO -.: Unusual Occurrence,

VR Voltage Reduction.

Source: International Affairs and Energy Emergencies, Form IE-417R, "Electric Power System Emergency Report." 


\section{Appendix B}

References

$105 / 106$ 


\section{Appendix B}

\section{References}

1. Energy Information Administration, Office of Energy Markets and End Use, Annual Energy Outlook, DOE/EIA-0383(90) (Washington DC, 1990).

2. Energy Information Administration, Oftice of Energy Markets and End Use, Assumptions for the Annual Energy Outlook, DOE/EIA-0527(90) (Washington DC, 1990).

3. Energy Information Administration, Office of Energy Markets and End Use, Annual Energy Review, DOE/EIA-0384(89) (Washington DC, 1990).

4. Bishop, Y.M.M. "Imputation, Revision, and Seasonal Adjustmunt," 1980 Proceedirgs of the American Statistical Association Meetings, July 1980.

5. Energy Information Administration, Office of Coal, Nuclear, Electric and Alternate Fuels, Cost and Quality of Fuels for Electric Utility Plants - Annual, DOE/EIA-0191(89), (Washington DC, 1990).

6. Energy Information Administration, Office of Coal, Nuclear, Electric and Alternate Fuels, Electric Power Monthly, DOE/EIA-0226(90/1-08), (Washington DC, 1990).

7. Hill E. and French C. "Editing Very '.uarge Data Bases," 1981 Conference on Information Sciences and Systems, The Johns Hopkins University, March 1981.

8. Energy Information Administration, Office of Coal, Nuciear, Electric and Alternate Fuels, Inventory of Power Plants in the United States, DOE/EIA-0095(89), (Washington DC, 1990).

9. Energy Information Administration, Office of Energy Markets and End Use, Monthly Energy Review, DOE/EIA-0035(90/1-08) (Washington DC, 1990).
10. Energy Information Adininistration, Office of Coal, Nuclear, Electric and Alternate Fuels, Electric Sales, Revenue, and Bills, DOE/EIA-0540(88), (Washington DC, 1990).

11. Energy Information Administration, Office of Statistical Standards, An Assessment of the Quality of Selected EIA Data Series: Electric Power Data, DOE/EIA-0292(89), (Washington DC, 1989).

12. Energy Information Administration, Office of Coal, Nuclear, Electric and Alternate Fuels, Annual Outlook for U.S. Electric Power, DOE/EIA-0474(85), (Washington DC, 1985); DOE/EIA-0474(89), (Washington DC, 1989); DOE/EIA-0474(90), (Washington DC, 1990).

13. Energy Information Administration, Office of Coal, Nuclear, Electric and Alternate Fuels, Financial Statistics of Investor-Owned Electric Utilities, DOE/EIA-0437(89)/1, (Washington DC, 1990).

14. Energy Information Administration, Office of Coal, Nuclear, Electric and Alternate Fuels, Financial Statistics of Publicly Owned Electric Utilities, DOE/EIA-0437(89)/2, (Washington DC, 1990).

15. Energy Information Administration, Office of Ccal, Nuclear, Electric and Alternate Fuels, Electric Plant Cost and Power Production Expenses, DOE/EIA-0455(88), (Washington DC, 1990).

16. Energy Information Administration, Office of Coal, Nuclear, Electric and Alternate Fuels, Electric Trade in the United States, DOE/EIA-0531(86), (Washington DC, 1990).

17. Energy Information Administration, Office of Energy Markets and End Use, State Energy Data Report, DOE/EIA-0214(88) (Washington DC, 1990). 


\section{Appendix C}

Technical Notes 


\section{Appendix C}

\section{Technical Notes}

\section{Sources of Data}

The E'ectric Power Annual is prepared by the Electric Power Livision; Office of Coal, Nuclear, Electric and Alternate Fuels; Energy Information Administration (EIA); U.S. Department of Energy (DOE). Data published in the Electric Power Annual are compiled from two statistical forms filed monthly and seven forms filed annually by electric utilities. Those forms are: the Form ElA-759, "Monthly Power Plant Report"; the FERC Form 423, "Monthly Report of Cost and Quality of Fuels for Electric Plants"; the Form EIA-861, "Annual Electric Utility Report"; the Form EIA-860, "Annual Electric Generator Report"; the Federal Energy Regulatory Commission (FERC) Form 1, "Annual Report of Major Electric Utilities, Licensees, and Others"; the Form EIA-4:2, "Annual Report of Public Electric Utilities"; the Form EIA-767, "Steam-Electric Plant Operation and Design Report"; the Department of Energy Form IE-411, "Coordinated Bulk Power Supply Program"; and the Department of Energy, Form FE-781R, "Annual Report of International Electric Import/Export Data." In addition, the Flectric Power Annual also includes data collected on the Form IE-417R, "Electric Power System Emergency Report." A brief summary of each form is presented below.

\section{Form EIA-759}

The Form EIA-759 is a mandatory census of all operators of electric utility plants produc ing electric power for public use. The Form EIA-759 is used to collect monthly data on net generation, consumption of coal, petroleum, and natural gas; and end-of-the-month stocks of coal and petroleum for each plant by prime mover and fuel-type combination. Summary data from the Form EIA-759 are also published in the Electric Power Monthly (EPM), the Monthly Energy Review: (MER), Annual Outlook for U.S. Electric Power(AOEP). and the Annual Energy Review (AER). These reports present aggregated data for electric utilities at the U.S., Census division, and North American Electric Reliability Council Region (NERC) levels.
Instrument and Design History. Prior to 1936, the Bureau of the Census and the U.S. Geological Survey collected, compiled, and published data on the electric power industry. In 1936, the Federal Power Commission (FPC) assumed all data collection and publication responsibilities for the electric power industry and implemented the FPC Form 4. The Federal Power Act, Sections 311 and 312, and FPC Order 141 define the legislative authority to collect power production data. The Form EIA-759 replaced the FPC Form 4 in January 1982.

Data Processing. The Form EIA-759, along with a return envelope, is mailed to respondents approximately 4 working days before the end of the month. The respondents names are obtained from a computerized mailing address file. The completed forms are to be returned to the EIA by the 10th day after the end of the reporting month. After receipt, data from the completed forms are manually logged in and edited before being keypunched for automatic data processing. An edit program checks the data for errors not found during maisual editing. The electric utility companies are telephoned to obtain data in cases of missing reports and to verify data when questions arise during editing. Following verification of the data, text and tables of aggregated data are produced for inclusion in the EPM. Following EIA approval of the EPM, the data are made available for public use, on a costrecovery basis, through custom computer runs, data tapes, or in publications.

\section{FERC Form 423}

The FERC Form 423, a lestricted-universe census, is a monthly record of delivered-fuel purchases, submitted by approximately 225 electric utilities for each plant with a fossil-fueled steam-electric nameplate capacity of 50 or more megawatts. Summary data from the FERC Form 423 are also published in the EPM, the $M E R$, and the Cost and Quality of Fuels for Electric Utility Plants - Annual. These reports present aggregated data on electric utilities at the U.S. and Census division level.

Instrument and Design History. On July 7, 1972, the FlC iswicd Order Number 453 enacting the New Code of Federal Regulations. Section 141.61, legally creating 
the Form 42: Originally, the form was used to collect data only on fossil-steam plants, but was amended in 1974 to include data on internal combustion and combustion turbines. The FERC Form 423 replaced the FPC Form 423 in January 1983. Peaking units, which were previously collected on the FPC Form 423 were eliminated from the FERC Form 423. In addition, the nameplate capacity threshuid was changed from 25 megawatts to 50 megawatts. This reduction in coverage eliminated approximately 50 utilities and 250 piants. All FPC Form 423 historical data in this publication have been revised to reflect the new nameplate capacity threshold of 50 or more megawatts.

Data Processing. A computerized mailing-address file is used to send a 6-month supply of forms to respondents semiannually. Completed forms are to be returned to the EIA by the 45th day following the end of the reforting month. Data from the completed forms are manually logged in and edited before being keypunched for automatic data processing. The electric uiility companies are telephoned to obtain data in cases of missing reports and to verify data when questions arise during editing. Following verification of the data, text and tables of aggregated data are produced for inclusion in the EPM. After the EPM receives EIA approval, the data become available for public use, on a cost-recovery basis, through custom computer runs, data tapes, or in publications.

\section{Form EIA-861}

The Form EIA-861 is a mandatory census of electric utilities in the United States, its territories, and Puerto Rico. The Form ElA-861 data contained in this publication are for the United States only. The survey is used to collect information on power production and sales of electricity from approximately 3,250 electric utilities. The data collected are used to update the elec. tric vility frame data base maintained by the EIA. This data base supports queries from the Executive Branch, Congress, other public agencies, and the general public. Summary data from the Form EIA-861 are also contained in the EPM; the Electric Sales. Revenue, and Bills: the Financial Statistics of Selected Elestric Utilities; and the Annual Outlook for U.S. Electric Power. These reports present aggregate totals for electric utilities on a national level, by State, and by ownership type.

Instrument and Design History. The Form ElA-861 was implemented in January 1985 to collect data as of year-end 984. The Federal Administration Act of 1974 (Public Law 93-275) defines the legislative authority to collect these data.

Data Processing. The Form EIA-861 is mailed to the respondents in February to collect data as of the end of the preceding calendar year. The completed forms are 10 be returned to the EIA by May 1. The data are manually edited before being entered into the interac- tive on-line system. Interıal edit checks are performed to verify that current data total across and between schedules, and are comparable to data reported the previous year. Edit checks are also performed to compare data reported on the Form EIA-861 and similar data reported on the Forms EIA-826, "Monthly Electric Utility Sales and Revenue Report with State Distributions," the FERC Form 1, and the Form EIA-412, "Annual Repcrt of Public Electric Utilities." These are utility-level checks. Respondents are telephoned to obtain clarification of reported data and to obtain missing data.

Form EIA-86I figures shown in this publication differ comewhat from the sum of the monthly Form EIA-826 figures for sales publisher in the EPM and in issues of the Electric Power Annual prior to 1988. Both forms use sectoral classifications (residential, commercial, and industrial) based on the usual classification of consumers by electric utilities. At the national level, the Form EIA-861 and Form ElA-826 figures corresponded closely (withi: 3 percent) for all end-use sectors. Some larger differences were observed at the State level particularly for Tennessee due to a misclassification of commercial and industrial data in the Form EIA-826 survey.

Detailed comparisons and descriptions of conceptual problems affecting the quality of the data are discussed in the report, An Assessment of the Quality of Selected EIA Data Series: Electric Power Data. (See Appendix $B$ for the reference.) One example of such a problem inherent in data on electricity sales is that the etoral classification scheme can vary from utility to utility as influenced by the local public utility commission.

Differences between the Form EIA-861 and Form EIA-826 figures can result from the Form EIA-826 sampling procedure and/or from nonsampling error in either data collection. One source of nonsampling error in the Form EIA-826 stems from the practice of filing estimates when the actual figures are not yet available. Although respondents are instructed to file revisions, revisions are not always filed. In addition, the respondents have been instructed to review their Form EIA-826 figures at the end of the reporting year to ensure consistency with Form EIA-861 figures. The EIA compares thesc data series and attempts to resolve any differences. Nevertheless some reporting differences remain.

\section{Form EIA $\$ 60$}

The Form EIA 860 is a mandatory census of electric utilities in the United States that operate power plants or plan to operate a power plant within 10 year. of the reporting year. The survey is used to collect data on existing power plants from the electric utilities and their 10-year plans for constructing new plants, and modifying and retiring existing plants Data on the curvey are collected at the generating unit level. These 
data are then aggregated to provide totals by energy source (coal, petroleum, gas, water, uranium, other) and geographic area (State, NERC region, Federal region, Census division). Additionally, at the national level, data are aggregated to provide totals by prime mover. Data from the Form EIA-860 are also summarized in the Inventory of Power Plants in the United States, and are used as input to publications and studies by other offices in the DOE.

Instrument and Design History. The Form EIA-860 was implemented in January 1985 to collect rata as of year-end 1984. The Federal Energy Administration Act of 1974 (Public Law 93-275) defines the legislative authority to collect these data.

Data Processing. The Form EIA-860 is mailed to approximately 900 respondents in December and the completed forms are to be returne's to the EIA by February 15 containing data as of the end of the preceding calendar year. Data for each respondent are preprinted from the applicable data base. Respondents are instructed to verify all preprinted data and to supply missing data. The data are manually edited before being keyed for automatic data processing. Computer programs containing additional edit checks are run. Respondents are telephoned to obtain correction or clarification of reported data and to obtain missing data, as a result of the manual and automatic editing process.

\section{FERC Form 1}

The FERC Form 1 is a mandatory restricted-universe census of major investor-owned electric utilities having, in each of the last 3 consecutive years, sales or transmission service that exceeds any one or more of the following: (1) 1 million megawathours of total sales, (2) 100 megawat hours of sales for resale, (3) 500 megawatthours of gross interchange out, or (4) 500 megawatthours of wheeling for others (deliveries plus losses). All major investor-owned electric utilities, licensees, or others subject to the Federal Power Act of 1935 must submit this form annually to the FERC through the EIA. Classification of such entities is provided in the FERC Uniform System of Accounts Prescribed for Public Utilities and Licensees Subject to the Provisions of the Federal Power Act. Approximately 180 electric utilities are classified as major. Excluded from EIA publications are Northeast Nuclear Energy Company, whose data were not included to prevent double counting of data for the Millstone nuclear plant, which Northeast Nuclear Energy Company operates for Connecticut Light \& Power Company and Western Massachusetts Electric Company; and Golden Spread Electric Cooperative, whose data were not included since it is classified as a cooperative electric utility on the Form EIA-861. The FERC, however, has determined that Golden Sprea, Electric Cooperative should file a FERC Form 1 urider Section

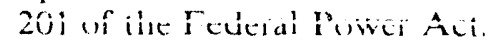

The FERC. Form 1 is used to collect data on income and earnings, taxes, depreciation and amortization, distribution of salaries and wages, electric operating revenues, electric maintenance expenses, generating plant statistics, planned construction data, year-end balance sheets, and general corporate information. Respondents are required to report data on historical plant cost and power production expenses for their hydroelectric plants with a generator nameplate capacity of 10 or more megawatts each steam-electric plant with a generator nameplate capacity of 25 or more megawatts; and each gas-turbine plant with a generator nameplate capacity of 10 or more megawatts. Less detailed data are required for other plants.

Instrument and Design History. The Federal Power Commission's (FPC) Form 1, the predecessor of the FERC Form 1, was implemented in 1935 by the FPC. When the FPC was merged with the DOE in October 1977, the collection and processing of data on the form became the responsibility of the EIA. This mandatory survey is conducted in accordance with the FERC Uniform System of Accounts for Private Utilities and Licensees.

Data Processing. The completed forms are to be returned to the EIA on or before April 30, containing data for the preceding calendar year. Manual editing of the reported data is completed prior to data entry. Additional edit checks of the data are performed through computer programs. The program edits include both deterministic checks, in which records are checked for the presence of data in required fields, and statistical checks, in which the data are checked against a range of values based on historical data values and for logical or mathematical consistency with data elements reported in the form. Discrepancies found in the data, as a result of these checks, are resolved either by the processing office or by further information obtained from a telephone call to the respondent company.

\section{Form EIA-412}

The Form EIA-412 is a restricted-universe census used annually to collect accounting, financial, and operating data from publicly owned electric utilities in the United States. Those publicly owned electric utilities engaged in the generation, transmission, or distribution of electricity which had 120,000 megawatthours of sales to ultimate consumers and/or 120,000 megawatthours of sales for resale for the 2 previous years, as reported on the Form ElA-861, "Annual Electric Utility Report," must submit the Form EIA-412. The criteria used to select the respondents for this form results in approximately 450 publicly owned utilities.

Federal electric utilities are required to file the Form EIA-4!2. The financial data for the U.S. Army Corps of Engineers (except for Saint Mary's Falls at Sault St. Maric, Michigan); the U.S. Internationa! Boundary and 
Water Commission; and the U.S. Department of Interior. Bureau of Reclamation were collected on the Form EIA-412 from the Federal power marketing administrations.

Data on plant construction cost, production expenses, generating equipment characteristics, and other relevant operating data are also published in Electric Plant Cost and Power Production Expenses.

Instrument and Design History. The FPC created the Form IM in 1961 as a mandatory survey. It became the responsibility of the EIA in October 1977 when the FPC was merged with DOE. In January 1980, the Form $1 \mathrm{M}$ was superseded by the Form EIA-412, also a mandatory survey. Data currently reported on the Form EIA-412 are the same as that previously reported on the FPC Form $1 \mathrm{M}$.

Data Processing. The processing of data reported on this form is the responsibility of the Data Systems Branch, Electric Power Division of the Office of Coal, Nuclear, Electric and Alternate Fuels. The completed forms are due in this office on or before April 30. Nonresponse follow-up procedures are used to attain 100-percent response. Automated systems are used to edit data from the surveys using deterministic and statistical checks. When all data have passed the edit process, the data are aggregated into a master file that is used as input to the publication.

\section{Form EIA-767}

The Form EIA-767 is a mandatory restricted-universe census of all electric power plants with a total existing or planned organic- or nuclear-fueled steam-electric generator nameplate rating of 10 or more megawatts. The entire form is filed by approximately 700 power plants with a nameplate capacity of 100 or more megawatts. An additional 200 power plants with a nameplate capacity between 10 and 100 megawatts submit information only on fuel consumption/quality, boiler/ generator configuration, and flue-gas desulfurization equipment, if applicable. The Form EIA-767 is used to collect data annually on plant operations and equipment design (including boiler, generator, cooling system, flue gas desulfurization, flue gas particulate collectors, and stack data). Data from the Form EIA-767 are used for economic, regulatory, and environmental analyses conducted by the DOE, the FERC, the Environmental Protection Agency, and the Department of Commerce.

Instrument and Design History. The Federal Energy Administration Act of 1974 (Public Law 93-275) defines the legislative authority to collect these data. The predecessor form, FPC-67, "Steam-Electric Plant Air and Water Quality Control Data," was used to collect data from 1969 to 1980, when the form number was changed to Form EIA.767. In 1982, the form was completely redesigned and given the name Form EIA-767,
"Steam-Electric Plant Operation and Design Report." In 1986, the respondent universe of 700 was increased to 900 to include plants with nameplate capacity from 10 megawatts to 100 megawatts. Respondents for thuse 200 additional plants complete only pages $1,5,6$, and, if applicable, 13, and 14.

Data Processing. The Form EIA-767 is mailed to respondents in January to collect data as of the end of the preceding calendar year. The completed forms are to be returned to the EIA by May l. Equipment design data for each respondent are preprinted from the applicable data base. Respondents are instructed to verify all preprinted data and to supply missing data. The data are manually reviewed before being keyed for automatic data processing. Computer programs containing additional edit checks are run. Respondents are telephoned to obtain correction or clarification of reported data and to obtain missing data, as a result of the manual and automatic editing process.

\section{Form IE-411}

The Form IE-411 is filed annually as a voluntary report. The information reported includes: (1) actual energy and peak demand for the preceding year and 10 additional years; (2) existing and future generating capacity; (3) scheduled capacity transfers; (4) projections of capacity, demand, purchases, sales, and scheduled maintenance; (5) assessment of adequacy; (6) generating capacity unavailability; (7) bulk power system maps; (8) near term transmission adequacy; (9) future critical bulk power facilities that may not be in service when required; and, (10) system evaluation criteria. These data support queries from the executive branch, Congress, other public agencies, and the general public. These reports present various council aggregate totals for their member electric utilities, with some nonmember information included.

Instrument and Design History. The Form IE-411 program was initiated under the Federal Power Commission Docket R-362, reliability and adequacy of electric service, and Orders 383-2, 383-3, and 383-4. The Department of Energy, established in October 1977, assumed the responsibility for this activity. This form is considered voluntary under the authority of the Federal Power Act (Public Law 88-280), The Federal Energy Administration Act of 1974 (Public Law 93-275), and the Department of Energy Organization Act (Public Law 95-91). It is now under the jurisdiction of the Assistant Secretary for International Affairs and Energy Emergencies.

Data Processing. The Form IE-411 is filed annually on April 1 by the nine North American Electric Reliability Councils. The forms are compiled from data furnished by electric utilities within the council areas. Data from the Form IE-411 are also summarized in the staff report Electric Power Supply and Demand for the Contiguous United States (DOE/IE-(0)13) 


\section{Form 1E-417R}

Electric utilities or other entities, subject to the provisions of Section 311 of the Federal Power Act (FPA), that are engaged in the generation, transmission, or distribution of electric energy for delivery and/or sale to the public are required to report expeditiously any 1) loss of firm system loads; 2) voltage reductions and public appeals; 3) vulnerabilities that could impact bulk electric power system adequacy or reliability; and, 4) fuel supply emergencies to the DOE.

In accordance with Section 202(a) of the Federal Power Act (FPA), the DOF is responsible for encouraging actions to assure an abundant supply of electric energy throughout the country. Under Section 311 of the FPA, the DOE is authorized and directed to collect information regarding the generation, transmission, and distribution of electric energy and to report the problems and developments of the electric utility industry 10 Congress. The Secretary of Energy has the Federal responsibility of receiving reports of major electric utility system emergencies. The Secretary has delegated that responsibility to the Office of International Affairs and Energy Emergencies (IE) with the DOE.

Instrument and Design History. The collection of outage data was initiated by the FPC prior 10 the organization of the DOE. After Congress passed legislation creating the DOE, the collection of electric power system outage data became a function of the DOE. Currently the Assistant Secretary of $I E$ is the principal DOE office for this activity. Form IE-417 was activated after public comment on a rule-making procedure (FR 7/6/83). The form was revised to Form IE-417R after public comment under a later rulemaking procedure (FR 10/31/86).

Data Processing. Reports of emergencies are usually received by the Alert Coordination Officer via telephone. The Director. Office of Emergency Operations, has the authority to require a full technical report (after notice in the Federal Register).

\section{Form FE-781R}

The Form FE-781R, "Annual Report of International Electrical Export/Import Data" is used to collect on an annual basis, monthly information on the gross amounts of electrical energy received and delivered and the costs and revenue associated with these transactions. The use of the format contained in Form FE-781R is optional for reporting purposes; however, submissicn of the data is mandatory.

Instrument and Design History. The authority to issue presidential permits pursuant to Executive Order Number 10485 was transferred to the Secretary of Energy by Executive Order Number 12038 (43 FR 4957 February $;$, 1987). This responsibility was delegated by the Secretary to the Economic Regulatory Administration (DOE Delegation Order Number 0204-04, October 1, 1977). The authority was redelegated (DOE Delegation Order Number 127) to the Office of Fuels Programs, Fossil Energy, (54 FR 11436 March 20, 1989). The survey universe is defined under Title 10 of the Code of Federal Regulations, Secrions 205.308 and 205.325 to include all public utilities or other entities subject to the Department of Energy jurisdiction under Part II of the Federal Power Act engaged in the export of electric energy across the international borders of the United States with Canada and Mexico. It also includes those engaged in the transmission of electrical energy across these borders who hold a presidential permit.

Data Processing. The Form FE-781R is mailed to the respondents to collect annually, the monthly data for the preceding calendar year. The completed forms are to be returned to the DOE by February 15. The receipts are manually edited and the data used for the Presidential Permit Program are entered into a machine readable format.

\section{Quality of Data}

The Office of Coal, Nuclear, Electric and Alternate Fuels (CNEAF) is responsible for routine data improvement and quality assurance activities. All operations in this office are done in accordance with formal standards established by the EIA. These standards are the measuring rod necessary for quality statistics. Data improvement efforts include verification of data-keyed input by automatic computerized methods, editing by subject matter specialists, and follow up on nonrespondents. The CNEAF office supports the quality assurance efforts of the data collectors by providing advisory reviews of the structure of information requirements, and of proposed designs for new and revised data collection forms and systems. Once implemented, the actual performance of working data collection systems is also validated. Computerized respondent data files are checked to identify those who fail to respond to the survey. By law, nonrespondents may be fined or otherwise penalized for not filing a mandatory EIA data form. Before invoking the law, the EIA tries to obtain the required information by encouraging cooperation of nonrespondents.

Completed forms received by the CNEAF office are sorted, screened for completeness of reported information, and keyed onto computer tapes for storage and transfer to random access data bases for computer processing. The information coded on the computer tapes is manually spot-checked against the forms to certify accuracy of the tapes. To ensure the quality standards established by the EIA, formulas that use the past history of data values in the data base have been designed and implemented to check data input for errors auto- 
matically. Data values that fall outside the ranges prescribed in the formulas are verified by telephoning respondents to resulve any discrepancies.

Conceptual problems affecting the quality of data are discussed in the report, An Assessment of the Quality of Selected EIA Data Series: Electric Power Data. This report is published by the Energy Information Administration (Office of Statistical Standards, see Appendix B).

\section{Data Editing System}

Data from the form surveys are edited using automated systems. The edit includes both deterministic checks, in which records are checked for the presence of required fields and their validity; and statistical checks, in which estimation techniques are used to validate data according to their behavior in the past and in comparison to other current fields.

\section{Confidentiality of the Data}

The data collected on the forms used for input to this report are not confidential.

\section{Rounding Rules for Data}

Given a number with $r$ digits to the left of the decimal and $d+t$ digits in the fraction part, with $d$ being the place to which the number is to be rounded and $t$ being the remaining digits which will be truncated, this number is rounded to $r+d$ digits by adding 5 to the $(\mathrm{r}+\mathrm{d}+1)$ th digit when the number is positive or by subtracting 5 when the number is negative. The t digits are then truncated at the $(\mathrm{r}+\mathrm{d}+1)$ th digit. The symbol for a rounded number truncated to zero is $\left(^{*}\right)$.

\section{Data Correction and Revision Procedures}

The CNEAF office has adopted the following policy with respect to the revision and correction of data published in energy data reports and made available in a machine format:

1. All data collected by this office (excluding secondary source data) will be published as preliminary data when first appearing in an energy data report.

$\angle$ The next publication of the data will be in final form at which point the data base is closed.

3. Revisions or corrections will be made only in the event a substantial (greater than 1 percent) reporting change is received or a substantial (greater than 1 percent) error is discovered.

4. Revisions or corrections to published secondary source data will be made if and when the organization from which the data were received revises or corrects the data.

5. No revisions will be made without the approval of the Director, Electric Power Division.

The Electric Power Annual presents the most current annual data available to the EIA. The statistics may differ from those published previously in the Electric Power Monthly due to corrections, revisions, or other adjustments that may have been made to the data subsequent to the original monthly submissions (Table C1). Data for emissions from fossil-fueled steamelectric generating units in this publication have been revised to 1) include petroleum coke, 2) reflect refinements to the calculation methodology, and 3) rectify minor reporting errors. These revisions represent increases of 0.3 percent for sulfur dioxide, 2.5 percent for nitrogen oxides, and 0.2 percent for carbon dioxide at the national level. The sales of electricity to ultimate consumers, its associated revenue, and the number of ultimate consumers for 1988 have been revised for the States of Missouri and Alaska. These revisions represent a .04 and -.49 percent change in sales of electricity to ultimate consumers, a .03 and -.27 percent change in revenue, and a .04 and -.44 percent change in the number of ultimate consumers, respectively. Data for the average cost of coal have been revised to incorporate substantial revisions submitted by a respondent. These revisions represent an increase in the cost of coal delivered to the State of Georgia of 1.5 percent, to the South Atlantic Census Division of 0.3 percent, and at the national level of 0.1 percent. 
Table C1. Comparison of Prior Versus Final Published Data at the National Level, 1989

\begin{tabular}{|c|c|c|c|}
\hline Item & Prior & Final & $\begin{array}{l}\text { Percent } \\
\text { Change }\end{array}$ \\
\hline \multicolumn{4}{|l|}{ Generation (gigawatthours) } \\
\hline 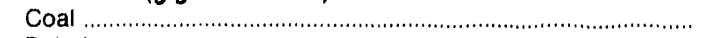 & $1,551,852$ & $1,553,661$ & 0.12 \\
\hline 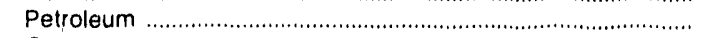 & 158,241 & 158,318 & .05 \\
\hline 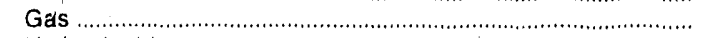 & 264,957 & 266,598 & 62 \\
\hline 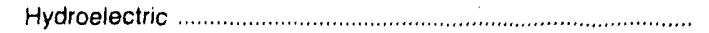 & 265,061 & 265,063 & 0 \\
\hline Nuclear & 529,355 & 529,355 & 0 \\
\hline Other ${ }^{1} \ldots \ldots \ldots \ldots$ & 11,309 & 11,309 & 0 \\
\hline \multicolumn{4}{|l|}{ Consumption } \\
\hline Coal $(1,000$ short tons $)$ & 765,820 & 766,888 & .14 \\
\hline 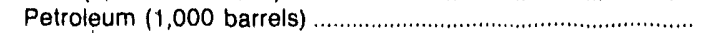 & 266,836 & 267,451 & .23 \\
\hline Gas (million cubic feet) & $2,767,903$ & $2,787,012$ & .69 \\
\hline \multicolumn{4}{|l|}{ Stocks ${ }^{2}$} \\
\hline 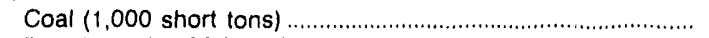 & 135,894 & 135,860 & -.03 \\
\hline Petroleum $(1,000$ barrels $)$ & 61,410 & 61,270 & -.23 \\
\hline \multicolumn{4}{|l|}{ Recelpts } \\
\hline Coal $(1,000$ short tons $)$ & 753,217 & 753,217 & 0 \\
\hline Petroleum $(1,000$ barrels) & 246,422 & 246,422 & 0 \\
\hline Gas (million cubic feet) & $2,472,506$ & $2,472,506$ & 0 \\
\hline \multicolumn{4}{|l|}{ Average Cost (cents per million Btu) } \\
\hline 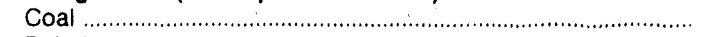 & 144.5 & 144.6 & .07 \\
\hline Petroleum & 289.3 & 289.3 & 0 \\
\hline $\mathrm{Ga} ; \mathrm{A}$ & 235.5 & 235.5 & 0 \\
\hline
\end{tabular}

Includes geothermal, wood, wind, waste, and solar.

2 Stocks are shown as of December 31 of each year

Nole: Percent change is calculated before rounding.

Sources: Energy Information Administration. "Prior Generation, Consumption, and Stocks Data: "Electric Power Monthly" DOE/EIA-0226(89/01. 12). -Prior Recelpts and Costs Data: Cost and Quality of Fuels for Electric Utility Plants DOE/EIA-0191(B9), -Final Generation, Consumption, and Stocks Data: Form EIA-759, "Monthly Power Plant Report." -Final Receipts and Costs Data: Federal Energy Regulatory Commission, FERC Form 423, "Monthly Report of Cost and Quality of Fuels for Electric Plants."

\section{Formulas and Calculations}

\section{Average Heat Content}

In order to determine the Btu value per unit of consumption (Table C2) for each of the fossil fuels collected on the Form EIA-759, the heat content values contained on the FERC Form 423 were used. Data on the FERC Form 423 represent 86 percent of the total generator nameplate capacity for all electric utilities.

\section{Percent Difference}

The following formula is used to calculate percent differences.

Percent Difference $=\left(\frac{x\left(t_{2}\right)-x\left(t_{1}\right)}{x\left(t_{1}\right)}\right) \times 100$,

where $x\left(t_{1}\right)$ and $x\left(t_{2}\right)$ denote the quantity at year $t_{1}$ and subsequent year $t_{2}$

\section{Form EIA-759}

Data for the Form EIA-759 are collected at the plant level. These data are then aggregated to provide geographic totals at the State, Census division, and U.S. level, or totals by type of plant. Consumption of fuel(s) is converted from quantities (in short tons, barrels, or thousand cubic feet) to Btu at the plant level. Endof-month fuel stocks for a single generating plant may not equal beginning-of-the-month stocks, plus receipts, less consumption, for many reasons, including the fact that several plants may share the same fuel stock.

\section{FERC Form 423}

Data for the FERC Form $4 ? 3$ are collected at the plant level. These data are then used in the following formulas to produce aggregates and averages for each fuel type at the State, Census division, and U.S. level. For these formulas, receipts and average heat content are at the plant level. For each geographic region, the summation $\Sigma$ represents the sum of all plants in that geographic region. Additionally,

- For coal, units for receipts $(R)$ are in tons, units for average heat content $(A)$ are in Btu per pound, and the unit conversion $(U)$ is 2,000 pounds per ton; 
Table C2. Average Heat Content of Fossil-Fuel Recelpts by Census Division and State, 1989

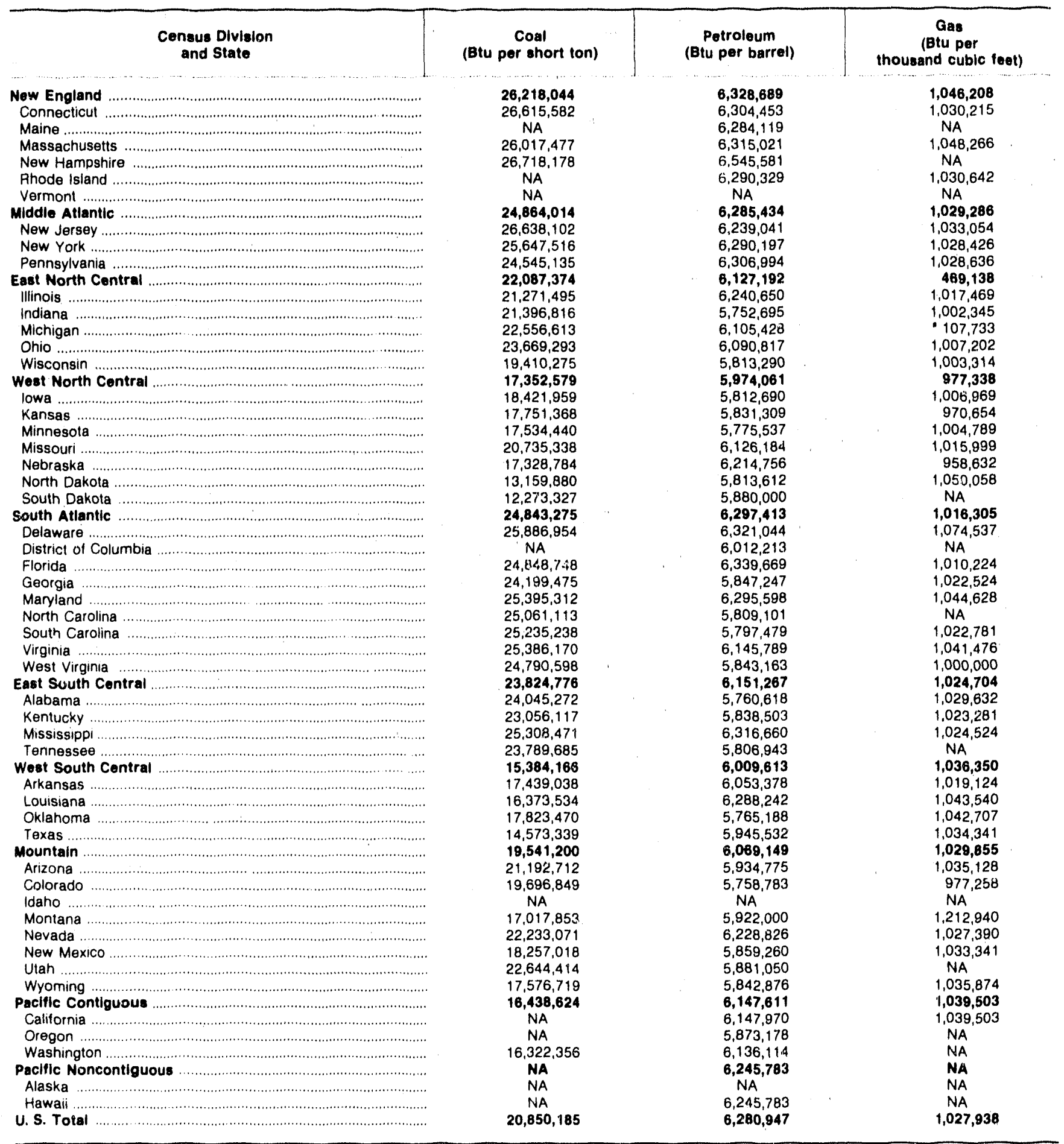

- Consists mostly of blast furnace gas, which has an average heat content of 85,472 Btu per thousand cubic feet. For further detailed information, see Table 37 of the "Electric Power Monthiy."

NA :- Not applicable.

Note: Totais may not equal sum of components because of independent rounding.

Source: Federal Energy Regulatory Commission, FERC Form 423, "Monthly Report of Cost and Quality of Fuels for Electric Plants." 
- For petroleum, units for receipts $(R)$ are in barrels, units for average heat content $(A)$ are in Btu per gallon, and the unit conversion ( $U$ ) is 42 gallons per barrel;

- For gas, units for receipts $(R)$ are in thousand cubic feet (Mcf), average heat content $(A)$ are in Btu per cubic foot, and the unit ronversion ( $U$ ) is 1,000 cubic feet per Mcf.

Total Btu $=\sum_{i}\left(R_{i} \times A_{i} \times U\right)$

where $i$ denotes a plant; $R_{i}=$ receipts for plant $i$; $A_{i}=$ average heat content for receipts at plant $i$; and, $U=$ unit conversion;

Weighted Average Btu $=\frac{\sum_{i}\left(R_{i} \times A_{i}\right)}{\sum_{i} R_{i}}$,

where $i$ denotes a plant; $R_{i}=$ receipts for plant $i$; and, $A_{i}=$ average heat content for receipts at plant $i$.

The weighted average cost in cents per million Btu is calculated using the following formula:

Weighted Average cost $=\frac{\sum_{i}\left(R_{i} \times A_{i} \times U \times C_{i}\right)}{\sum_{i}\left(R_{i} \times A_{i}\right)}$,

where $i$ denotes a plant; $R_{i}=$ receipts for plant $i$; $A_{i}=$ average heat content for receipts at plant $i$; and, $C_{i}=$ cost in cents per million Btu for plant $i$.

The weighted average cost in dollars per unit is calculated using the following formula:

Weighted Average Cost $=\frac{U \sum_{i}\left(R_{i} \times A_{i} \times C_{i}\right)}{\left(10^{8} \frac{\text { cents }}{\text { dollar }}\right) \sum_{i} R_{i}}$

where $i$ denotes a plant; $R_{i}=$ receipts for plant $i$; $A_{i}=$ average heat content for receipts at plant $i$; $U=$ unit conversion; and, $C_{i}=$ cost in cents per million Btu for plant $i$.

\section{Form EIA-861}

Data for the Form EIA-861 are collected at the utility level from all electric utilities in the United States, its territories, and Puerto Rico. Form EIA-861 data in this publication are for the United States only. These data are then aggregated to provide geographic totals at the State, NERC Region, Census division, and national level. Sources and disposition of data are also provided by utility class of ownership and retail consumer class of service. Average revenue per kilowatthour of electricity sold is calculated by divid- ing total annual retail revenue by the total annual retail sales of electricity.

\section{Form EIA-860}

Data for the Form EIA-860 are submitted at the generating unit level and are then aggregated to provide totals by energy source and geographic area. In addition, at the national level, data are aggregated by prime mover.

Estimated values for net summer and net winter capability for electric generating units were developed by use of a regression formula. Estimates of net summer capability and net winter capability are made for operable nonnuclear electric generating units with no reported capability and for all electric generating units that are under construction or in the planning stage that are not nuclear-powered or coal-fired. The formula is based on reported values in the 1984 data file. The formula is used to estimate values for existing units where data are missing and for projected units. In all formulas, the symbol, ${ }^{*}$, is an operator meaning multiplied by; the symbol, **, is an operator meaning raised to the specified power (exponent).

For nonnuclear units,

Net Summer Capability equals E**(i plus (ln(generator Nameplate Capacity)*(c)), rounded to nearest megawatt,

where

- $E$, the base of natural logarithms $(\ln )$, is approximately 2.71828 ,

- Generator Nameplate Capacity is expressed in megawatts,

- $i$ is the intercept, and $c$ is the regression coefficient,

- i equals 0.02564 , c equals 0.98423 , for steam units (Unit Type is Steam Turbine - Boiler (ST)),

- i equals 0.10255 , c equals 0.97841 , for gas-turbine units (Unit Types are Gas Turbine (GT) and Jet Engine (JE)),

- i equals 0.00397 , c equals 0.95914 , for combinedcycle units (Unit Types are Combined Cycle Steam Turbine with Supplemental Firing (CA), Combined Cycle - Single Shaft (CS), Combined Cycle Steam Turbine with Only Waste Heat Capability $(\mathrm{CW})$, and Combustion Cycle Combustion Turbine (CT)),

- i equals 0.08865 , c equals 1.00966, for internal combustion units (Unit Type is Internal Combustion (diesel) (IC)),

- i equals 0.04963 , c equals 1.03738 for conventional and pipeline hydroelectric units (Unit Types are Hydraulic Turbine (HC) and Hydraulic Turbine - Pipeline (HL)), 
- $\mathrm{i}$ equals 0.00922 , c equals 1.00630 , for pumped storage hydroelectric units (Unit Type is Hydraulic Turbine - Reversible (pumped storage) (HR)),

- $\mathrm{i}$ equals 0.02604 , c equals 0.98289 , for all other units (Unit Types are Fuel Cell (FC), Steam Turbine - Geothermal (GE), Ocean Thermal Turoine (OC), Photovoltaic (SP), Steam Turbine - Solar (SS), and Wind Turbine (WT)).

Net Winter Capability equals $\left(E^{* *}\right)^{*}$ (Generator Nameplate Capacity**), rounded to nearest megawatt,

where

- $E$, the base of natural logarithms $(\ln )$, is approximately 2.71828 ,

- Generator Nameplate Capacity is expressed in megawatts for conventional hydroelectric and pipeline hydroelectric units, and in kilowatts for all other unit types,

- $\mathrm{i}$ is the intercept, and $\mathrm{c}$ is the regression coefficient,

- $\mathrm{i}$ equals 0.1614 , c equals 0.98373 , for steam units (Unit Type is ST),

- i equals 0.2249, c equals 0.97881, for gas-turbine units (Unit Types are GT and JE),

- i equals 0.9626 , c equals 0.90413 , for combinedcycle units (Unit Types are CA, CS, CW, and CT),

- i equals 0.1378 , c equals 1.02501 , for internal combustion units (Unit Type is IC),

- i equals 0.0608 , c equals 1.02560 , for conventional and pipeline hydroelectric units (Unit Types are $\mathrm{HC}$ and $\mathrm{HL}$ ),

- i equals 0.1167 , c equals 1.08540 , for pumped storage hydroelectric units (Unit Type is HR),

- i equals 0.2859 , c equals 0.96587 , for all other units (Unit Types are FC, GE, OC, SP, SS, and WT).

\section{Composite Financial Indicators for Selected Investor-Owned Electric Utilities}

The following formulas are used to calculate composite financial indicators.

Electric Fixed Asset (Net Plant) Turnover =

$$
\frac{\sum_{i}\left(E O_{i}\right)}{\sum_{i}\left(L_{i}^{\prime}\right)}
$$

where $E O_{i}$ is the Electric Operating Revenue for the $i^{\text {th }}$ major utility, and $U_{i}$ is the Electric Utility Plant

-- Net for the $i^{\text {th }}$ major utility.

Total Asset Turnover $=$

$$
\frac{\sum_{i}\left(O_{i}\right)}{\sum_{i}\left(A_{i}\right)}
$$

where $O_{i}$ is the Operating Revenue for the $i^{\text {th }}$ major utility, and $A_{i}$ are the Total Assets for the $i^{\text {th }}$ major utility.

Current Assets to Current Liabilities =

$$
\frac{\sum_{i}\left(C A A_{i}\right)}{\sum_{i}\left(C A L_{i}\right)}
$$

where $C A A_{i}$ are the Current and Accrued Assets for the $i^{\text {th }}$ major utility, and $C A L_{i}$ are the Current and Accrued Liabilities for the $i^{t^{2}}$ major utility.

Long-Term Debt to Capitalization $=$

$$
\frac{\sum_{i}\left(L T D_{i}\right)}{\sum_{i}\left(C_{i}\right)} \times 100
$$

where $L T D_{i}$ is the Long-term Debt for the $i^{\text {th }}$ major utility, and $C_{i}$ is the Capitalization for the $i^{\text {th }}$ major utility.

Preferred Stock to Capitalization $=$

$$
\frac{\sum_{i}\left(P S_{i}\right)}{\sum_{i}\left(C_{i}\right)} \times 100
$$

where $P S_{i}$ is the Preferred Stock for the $i^{\text {th }}$ major utility, and $C_{i}$ is the Capitalization for the $i^{\text {th }}$ major utility.

Common Stock Equity to Capitalization =

$$
\frac{\sum_{i}\left(C S E_{i}\right)}{\sum_{i}\left(C_{i}\right)} \times 100
$$

when $C S E_{i}$ is the Common Stock Equity of the $i^{\text {th }}$ major utility; and, $C_{i}$ is the Capitalization for the $i^{\text {th }}$ major utility.

Total Debt to Total Assets $=$ 


$$
\frac{\sum_{i}\left(L T D_{i}+S T D_{i}\right)}{\sum_{i}\left(A_{i}\right)} \times 100
$$

where $L T D_{i}$ is the Long-term Debt of the $i^{\text {th }}$ major utility; $S T D_{i}$ is the Short-term Debt of the $i^{\text {th }}$ major utility; and, $A_{i}$ are the Total Assets of the $i^{\text {th }}$ major utility.

Common Stock Equity to Total Assets =

$$
\frac{\sum_{i}\left(C S E_{i}\right)}{\sum_{i}\left(A_{i}\right)} \times 100
$$

where $C S E_{i}$ is the Common Stock Equity of the $i^{\text {th }}$ major utility; and, $A_{i}$ are the Total Assets of the $i^{\text {th }}$ major utility.

Interest Coverage Before Taxes

Without AFUDC =

$$
\frac{\sum_{i}\left(\begin{array}{c}
I B I_{i}+E I T_{i}+G I T_{i} \\
+O U I T_{i}+T O I D_{i}-A C_{i}
\end{array}\right)}{\sum_{i}\left(I E_{i}\right)}
$$

where $I B I_{i}$ is Total Income Before Interest Charges for the $i^{\text {th }}$ major utility; $E I T_{i}$ are the Electric Income Taxes for the $i^{\text {th }}$ rnajor utility; $G I T_{i}$ are the Gas Income Taxes for the $i^{\text {th }}$ major utility; OUIT $i$ are the Other Utility Income Taxes for the $i^{t h}$ major utility; TOID ${ }_{i}$ are the Taxes for Other Income and Deductions for the $i^{\text {th }}$ major utility; $A C_{i}$ is the Allowance for Other Funds Used During Construction (AFUDC) for the $i^{\text {th }}$ major utility; and, $I E_{i}$ is the Interest Expense for the $i^{t h}$ major utility.

Profit Margin =

$$
\frac{\sum_{i}\left(N I_{i}\right)}{\sum_{i}\left(O_{i}\right)} \times 100
$$

where $N I_{i}$ is the Net Income of the $i^{\text {th }}$ major utility; and, $O_{i}$ is the Utility Operating Revenue for the $i^{\text {th }}$ major utility.

Return on Average Common Stock Equity $=$

$$
\frac{\sum_{i}\left(N I_{i}\right)}{\left(\sum_{i}\left(C S E B_{i}\right)+\sum_{i}\left(C S E E_{i}\right)\right)} / 2 \times 100,
$$

where $N I_{i}$ is the Net Income of the $i^{\text {th }}$ major utility; $C S E B_{i}$ is the Common Stock Equity at Beginning of Year, for the $i^{\text {th }}$ major utility, and CSEE is the Common Stock Equity at End of Year for the $i^{\text {th }}$ major utility.

Return on Investment $=$

$$
\frac{\sum_{i}\left(N I_{i}\right)}{\sum_{i}\left(A_{i}\right)} \times 100
$$

where $N I_{i}$ is the Net Income of the $i^{\text {th }}$ major utility; and, $A_{i}$ are the Total Assets of the $i^{t h}$ major utility.

\section{Composite Financial Indicators for Selected Publicly Owned Electric Utilities}

Electric Utility Plant per Dollar of Revenue =

$$
\frac{\sum_{i}\left(E U P_{i}\right)}{\sum_{i}\left(E O R_{i}\right)}
$$

where EUP is the Electric Utility Plant for the the $i^{\text {th }}$ public utility; and, EOR is the Electric Operating Revenue for the $i^{\text {th }}$ public utility.

Current Assets to Current Liabilities =

$$
\frac{\sum_{i}\left(C A_{i}\right)}{\sum_{i}\left(C L_{i}\right)}
$$

where $C A_{i}$ are the Current and Accrued Assets for the $i^{\text {th }}$ public utility; and, $C L_{i}$ are the Current and Accrued Liabilities for the $i^{\text {th }}$ public utility.

\section{Electric Utility Plant as a Percent of Total Assets $=$}

$$
\frac{\sum_{i}\left(E U P_{i}\right)}{\sum_{i}\left(T A_{i}\right)} \times 100
$$

where $E U P_{i}$ is the Electric Utility Plant for the $i^{\text {th }}$ public utility; and, $T A_{i}$ are the Total Assets for the $i^{t h}$ public utility.

Net Electric Utility Plant as a Percent of Total Assets =

$$
\frac{\sum_{i}\left(N E U P_{i}\right)}{\sum_{i}\left(T A_{i}\right)} \times 100
$$


where $N E U P_{i}$ is the Net Electric Utility Plant for the $i^{\text {th }}$ public utility; and, $T A_{i}$ is the Total isssets for th. $i^{\text {th }}$ public utility.

Accumulated Provision for Depreciation as a Percent of Electric Utility Plant $=$

$$
\frac{\sum_{i}\left(A P D_{i}\right)}{\sum_{i}\left(E U P_{i}\right)} \times 100
$$

where $A P D_{i}$ is the Accumulated Provision for Depreciation for the $i^{\text {th }}$ public utility; and, $E U P_{i}$ is the Electric Utility Plant for the $i^{\text {th }}$ public utility.

Electric Operation and Maintenance Expenses as a Percent of Electric Operating Revenue =

$$
\frac{\sum_{i}\left(E O M E_{i}\right)}{\sum_{i}\left(E O R_{i}\right)} \times 100
$$

where EOME $E_{i}$ is the Electric Operation and Maintenance Expenses for the $i^{\text {th }}$ public utility; and, $E O R_{i}$ is the Electric Operating Revenue for the $i^{\text {th }}$ public utility.

\section{Electric Depreciation and Amortization as a Percent of} Electric Operating Revenue $=$

$$
\sum_{i}^{i} \frac{\left(E D A_{i}\right)}{\left(E O R_{i}\right)} \times 100
$$

where $E D A_{i}$ is Electric Depreciation and Amortization for the $i^{\text {th }}$ public utility; and, EOR is the Electric Operating Revenue for the $i^{\text {th }}$ public utility.

Taxes and Tax Equivalents as a Percent of Electric Operating Revenue $=$

$$
\frac{\sum_{i}\left(T T E_{i}\right)}{\sum_{i}\left(E O R_{i}\right)} \times 100
$$

where $T T E_{i}$ are the Taxes and Tax Equivalents for the $i^{\text {th }}$ public utility; and, $E O R_{i}$ is the Electric Operating Revenue for the $i^{\text {th }}$ public utility.

Net Contributions and Services as a Percent of Electric Operating Revenue =

$$
\frac{\sum_{i}\left(C S_{i}\right)}{\sum_{i}\left(E O R_{i}\right)} \times 100,
$$

whure $C S_{i}$ are the Net Contributions and Services for the $i^{\text {th }}$ public utility; and EOR $R_{i}$ is the Electric Operating Revenue for the $i^{\text {th }}$ r ublic utility.

Interest Expense as a Percent of Electric Operating Revenue $=$

$$
\frac{\sum_{i}\left(I E_{i}\right)}{\sum_{i}\left(E O R_{i}\right)} \times 100
$$

where $I E_{i}$ is the Interest Expense for the $i^{\text {th }}$ public utility; and, $E O R_{i}$ is the Electric Operating Revenue for the $i^{\text {th }}$ public utility.

\section{Form EIA-767}

This section describes the methodology employed to calculate estimates of sulfur dioxide, nitrogen oxide, and carbon dioxide emissions using informa .on contained on Form EIA-767. The methodology used to calculate estimates of emissions has been verified by comparing the emissions data presented in this report with the National Acid Precipitation Assessment Program (NAPAP) data.

Uncontrolled Air Pollutant Emissions. Uncontrolled air pollutant emissions are those emissions that would occur in the absence of any control equipment. Uncontrolled sulfur dioxide, nitrogen oxide, and carbon dioxide emissions are determined by multiplying the quantity of fuel burned by an emission factor. An emission factor is the average quantity of pollutant released from a boiler when a unit of fuel is burned.

The source of both the sulfur dioxide and nitrogen oxides emission factors is the Environmental Protection Agency report, Compilation of Air Pollutant Emission Factors. ${ }^{31}$ Environmental Protection Agency emission factors are based on boiler type and fuel burned. The Environmental Protection Agency emission factors are used in Table C3. Emission factors for carbon dioxide were estimated by the Electric Power Division of the EIA. Emissions of carbon dioxide are generally a function of the amount of carbon in the fuel. Emission factors were estimated for fuel type independent of toiler type.

Uncontrolled emissions of sulfur dioxide and nitrogen oxides do not accurately depict the quantity of emissions released into the atmosphere because they fail to

\footnotetext{
${ }^{31}$ Environmental Protection Agency, Office of Air and Radiation, Supplement A to Compilation of Air Pollutant Emission Factors, Vol. I: Stationary Point and Area Sources, A P-42 (Research Triangle Park, North Carolina, October 1986).
} 
Table C3. Sulfur Dioxide, Nitrogen Oxide, and Carbon Dioxide Emission Factors

\begin{tabular}{|c|c|c|c|c|}
\hline \multirow[b]{2}{*}{ Fuel } & \multirow[b]{2}{*}{ Boller Type' } & \multicolumn{3}{|c|}{ Factors } \\
\hline & & $\begin{array}{c}\text { Sulfur } \\
\text { Dioxide }\end{array}$ & $\begin{array}{l}\text { Nitrogen } \\
\text { Oxides }^{3}\end{array}$ & $\begin{array}{l}\text { Carbon } \\
\text { Dioxide }\end{array}$ \\
\hline Coal" & & lbs per ton & Ibs per ton & Ibs per $10^{\circ}$ Btu \\
\hline Bituminous ................................... & $\begin{array}{c}\text { Pulverized Coal.-.Dry Bottom } \\
\text { Pulverized Coal.-.Wet Bottom } \\
\text { Cyclone } \\
\text { Spreader Stoker } \\
\text { All Others }\end{array}$ & $\begin{array}{l}39 \times 5 \\
39 \times 5 \\
39 \times 5 \\
39 \times 5 \\
39 \times 5\end{array}$ & $\begin{array}{r}21(15) \\
34 \\
37 \\
14 \\
7.5\end{array}$ & $\begin{array}{l}209 \\
209 \\
209 \\
209 \\
209\end{array}$ \\
\hline 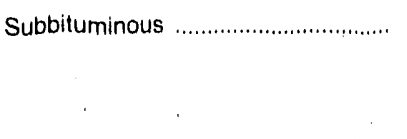 & $\begin{array}{c}\text { Pulverized Coal--Dry Bottom } \\
\text { Pulverized Coal--Wet Bottom } \\
\text { Cyclone } \\
\text { Spreader Stoker }\end{array}$ & $\begin{array}{l}35 \times S \\
35 \times S \\
35 \times S \\
35 \times S\end{array}$ & $\begin{array}{r}21(15) \\
34 \\
37 \\
14\end{array}$ & $\begin{array}{l}219 \\
219 \\
219 \\
219\end{array}$ \\
\hline 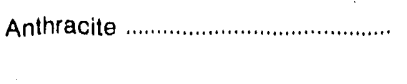 & $\begin{array}{c}\text { Pulverized'Coal } \\
\text { Stoker }\end{array}$ & $\begin{array}{l}39 \times S \\
39 \times S\end{array}$ & $\begin{array}{l}18 \\
10\end{array}$ & $\begin{array}{l}209 \\
209\end{array}$ \\
\hline 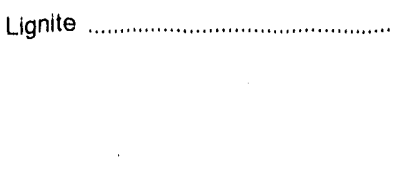 & $\begin{array}{l}\text { Pulverized Coal--Front Wall/Opposed Firing } \\
\text { Pulverized Coal--Tangential } \\
\text { Pulverized Coal--All Others } \\
\text { Cyclone } \\
\text { All Others }\end{array}$ & $\begin{array}{l}30 \times S \\
30 \times 5 \\
30 \times 5 \\
30 \times S \\
30 \times S\end{array}$ & $\begin{array}{r}14 \\
8 \\
12 \\
17 \\
6\end{array}$ & $\begin{array}{l}213 \\
213 \\
213 \\
213 \\
213\end{array}$ \\
\hline Petroleum & & Ibs per $10^{3} \mathrm{gal}$ & Ibs per $10^{3} \mathrm{gal}$ & Ibs per $10^{\circ}$ Btu \\
\hline Residual Heavy Oil ........................... & $\begin{array}{l}\text { Tangentially Fired } \\
\text { Vertically Fired } \\
\text { All Others }\end{array}$ & $\begin{array}{l}159.9 \times S \\
159.9 \times S \\
159.9 \times S\end{array}$ & $\begin{array}{r}42 \\
105 \\
67\end{array}$ & $\begin{array}{l}180 \\
180 \\
180\end{array}$ \\
\hline Light Distillate ..................................... & All. & $144.0 \times S$ & 20 & 156 \\
\hline Natural Gas .................................. & $\begin{array}{l}\text { Tangentially Fired } \\
\text { All Others }\end{array}$ & $\begin{array}{c}\text { Ibs per } 10^{\circ} \mathbf{c f} \\
0.6 \\
0.6\end{array}$ & $\begin{array}{l}\text { Ibs per } 10^{6} \text { of } \\
\qquad \begin{array}{c}275 \\
550\end{array}\end{array}$ & $\begin{array}{l}\text { Ibs per } 10^{6} \text { Btu } \\
\qquad \begin{array}{c}120 \\
120\end{array}\end{array}$ \\
\hline $\begin{array}{l}\text { 'See the Environmental Protecti } \\
2 \text { "XS" indicates that the constan } \\
\text { small quantities of sulfur trioxide anc } \\
3 \text { Parenthetic value is for tangen } \\
\text { fireball in an upward or don ard d } \\
\text { "Coal types are categorized by } \\
7.500 \text { to } 9,750 \text { Btu per pound), and } \\
\text { of }=\text { Cubic Feet. } \\
\text { gal }=\text { U.S. Gallons. } \\
\text { Ibs }=\text { Pounds. } \\
\text { S.jurce: Environmental Protect }\end{array}$ & $\begin{array}{l}\text { tency report for definitions of boller types. } \\
\text { t be multiplied by the percentage (by weight) } \\
\text { eous sulfates. } \\
\text { fired boilers. Tangentially fired boilers are } \\
\text { on. } \\
\text { ontent in this table as follows: Bltuminous (e } \\
\text { te (less than } 7,500 \text { Btu per pound). } \\
\text { gency Comoilation of Air Pollutant Emission }\end{array}$ & $\begin{array}{l}\text { in the fuel. Sul } \\
\text { the corners of } \\
\text { greater than } s\end{array}$ & $\begin{array}{l}\text { xide emission fa } \\
\text { tunace and are }\end{array}$ & $\begin{array}{l}\text { also account for } \\
\text { ble of directing the } \\
\text { Ituminous (equal to }\end{array}$ \\
\hline
\end{tabular}

reflect reductions prompted by emission standards. Consequently, controlled emissions are calculated to provide a more accurate estimate of actual emissions.

Controlled Sulfur Dioxide Emissions. Because of regulations controlling sulfur dioxide emissions, many utilities are required to install flue gas desulfurization (FGD) units at their coal-fired plants. ${ }^{32}$ FGD units typically remove between 70 and 90 percent of sulfur dioxide emissions. However, it is assumed that utilities operate them only to the degree necessary to meet the most stringent standard that they face. All utilities are required to comply with Federal standards, and some may also be required to comply with State and local standards. State and local standards may be more or less strict than Federal standards. In this study it is assumed that all utilities comply with the most stringent standard that they encounter. Accordingly, controlled sulfur dioxide emissions for each plant are calculated by subtracting the amount that it is required to remove in order to comply with its most stringent emission standard from its uncontrolled emissions level.

In order to calculate controlled sulfur dioxide emissions, plants are divided into five categories depending on whether their most stringent emission standard is: (1) more strict than 1978 Revised New Source Perfor. mance Standards (RNSPS); (2) equal to 1978 RNSPS; (3) less strict than 1978 RNSPS but more strict than 1971 NSPS; (4) equal to 1971 New Source Performance Standards (NSPS); or (5) less strict than 1971 NSPS. Plants built prior to 1971 are not required to comply

\footnotetext{
${ }^{32}$ Flue gas desulfurization units may also remove sulfur dioxide emissions from plants that burn both coal and oil.
} 
with either standard and therefore face only State and local requirements. These standards are reported on Form EIA-767. Plants built after August 17, 1971, but on or before September 18, 1978, must meet 1971 NSPS. Plants built after September 18, 1978, must meet 1978 RNSPS. Even if a plant is required to comply with NSPS or RNSPS, it still may face State or local standards.

The 1971 NSPS require all plants built after August 17,1971 , to emit no more than 1.2 pounds of sulfur dioxide per million Btu of heat input. Utilities are allowed to meet this standard by either ourning lowsulfur coal or using FGD units. The 1978 RNSPS are more complex. All plants built after September 18, 1978, are required to use FGD units. If they burn coal that has a sulfur content per million Btu (1) less than 1 pound, they must remove 70 percent of the sulfur dioxide; (2) between 1 and 3 pounds, they must remove the amount (between 70 and 90 percenc) that yields a sulfur dioxide emission rate of 0.6 pounds per million Btu; (3) between 3 and 6 pounds, they must remove 90 percent; and (4) greater then 6 pounds they must remove the amount that yields a sulfur dioxide emission rate of 1.2 pounds per million Btu.

Under the law, utilities are permitted to take credit for sulfur that remains in bottom ash (ash remaining in the bottom of the furnace after the coal is burned). For example, if a utility is required to remove 90 percent of the sulfur in the coal and 3 percent remains in the ash, it has to remove only 87 percent using scrubbers. This credit is included in emissions data in this report. It is likely, however, that in many cases the credit is not taken. In order to take the ash credit, utilities need to monitor the crial consumed on a caily basis; this is both time-consuming and costly. To the extent that utilities do not take the ash credit, emissions might be slightly overstated.

Controlled Nitrogen Oxide Emissions. Although there are many techniques that utilities can use to reduce their emissions of nitrogen oxides, it is impractical to calculate the actual reductions achieved. ${ }^{3}$ Consequently, two assumptions are made to determine estimates for controlled emissions of nitrogen oxides: all boilers subject to a standard for emissions of nitrogen oxides will emit at a rate equal to the standard; and all boilers that are not subject to a standard will emit the quantity of nitrogen oxides determined by using the emission factors of the Environmental Protection Agency. Accordingly, controlled emissions of nitrogen oxides are determined by choosing the lesser of two numbers: the emission standard or the uncontrolled emission rate.

A degree of complexity is added to this approach, however, because emission standards are not reported in consistent units. In some rare instances emission standards are reported in units that cannot be directly compared with estimated uncontrolled emission rates. Examples of such standards are ones that specify the concentration of nitrogen oxides allowed in the stack gas or the ambient quality concentration of nitrogen oxides (parts per million). In cases where these types of standards were reported, the uncontrolled emission rate was used. Such standards are very rare, however, and do not significantly affect the results.

Table C4. Unit-of-Measure Equivalents

\begin{tabular}{|c|c|c|c|}
\hline Unit & & Equivalent & \\
\hline $\begin{array}{l}\text { Kilowatt (kW) } \\
\text { Megawatt }(M W) \\
\text { Gigawatt }(G W) \\
\text { Terawatt }(T W)\end{array}$ & $\begin{array}{r}1,000 \\
1,000,000 \\
1,000,000,000 \\
1,000,000,000,000\end{array}$ & $\begin{array}{l}\text { (One Thousand) } \\
\text { (One Million) } \\
\text { (One Billion) } \\
\text { (One Trillion) }\end{array}$ & $\begin{array}{l}\text { Watts } \\
\text { Watts } \\
\text { Watts } \\
\text { Watts }\end{array}$ \\
\hline 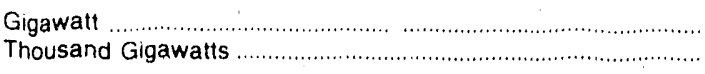 & $\begin{array}{r}1,000,000 \\
1,000,000,000\end{array}$ & $\begin{array}{l}\text { (One Miltion) } \\
\text { (One Billion) }\end{array}$ & $\begin{array}{l}\text { Kilowatts } \\
\text { Kilowatts }\end{array}$ \\
\hline $\begin{array}{l}\text { Kilowatthours (kWh) } \\
\text { Megawatthours (MWh) } \\
\text { Gigawatthours (GWh) } \\
\text { Terawatthours (TWh) }\end{array}$ & $\begin{array}{r}1,000 \\
1,000,000 \\
1,000,000,000 \\
1,000,000,000,000\end{array}$ & $\begin{array}{l}\text { (One Thousand) } \\
\text { (One Niiiion) } \\
\text { (One Biliion) } \\
\text { (One Trillion) }\end{array}$ & $\begin{array}{l}\text { Watthours } \\
\text { Watthours } \\
\text { Watthours } \\
\text { Watthours }\end{array}$ \\
\hline 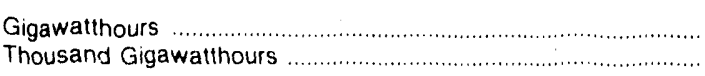 & $\begin{array}{r}1,000,000 \\
1,000,000,000\end{array}$ & $\begin{array}{l}\text { (One Million) } \\
\text { (One Billion) }\end{array}$ & $\begin{array}{l}\text { Kilowatthours } \\
\text { Kilowatthours }\end{array}$ \\
\hline 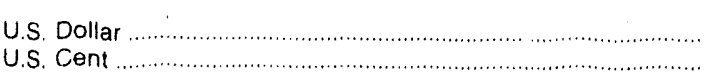 & $\begin{array}{c}1,000 \\
10\end{array}$ & $\begin{array}{l}\text { (One Thousand) } \\
\text { (Ten) Mills }\end{array}$ & Mills \\
\hline
\end{tabular}

Source: Energy Information Administration, Electric Power Division.

\footnotetext{
${ }^{13}$ Emissions of nitrogen oxides are highly sensitive (o many operating parameters that are not easily monitored
} 


\section{General Information}

\section{Use of the Glossary}

The terms in the glossary have been defined for general use. Restrictions on the definitions as used in these data collection systems are included in each definition when necessary to define the terms as they are used in this report.

\section{Obtaining Copies of Data}

Upon EIA approval of the Electric Power Annual, the data are available for public use on a cost-recovery basis.

Computer listings are obtained for a charge by submitting a written request to:
Energy Information Adrninistration, EI-541

Electric Power Division 1000 Independence Avenue

Washington, DC 20585

These data are also available monthly on machinereadable tapes. Tapes may be purchased by using Visa, MasterCard, or American Express cards as well as money orders or checks payable to the National Technical Information Service (NTIS). Purchasers may also use NTIS and Government Printing Office depository accounts. To place an order, contact:

National Technical Information Service (NTIS) Office of Data Base Services U.S. Department of Commerce 5285 Port Royal Road

Springfield, Virginia 22161

(703) $487-4650$ 


\section{Glossary}

Acid Rain; Also called acid precipitation or acid deposition, acid rain is precipitation containing harmful amounts of nitric and sulfuric acids formed primarily by nitrogen oxides and sulfur oxides released to the atmosphere when fossil fueis are burned. It can be wet precipitation (rain, snow, or fog) or dry precipitation (absorbed gaseous and paiticulate matter, aerosol particles or dust). Acid rain has a pH below 5.6. Normal rain has a $\mathrm{pH}$ of about 5.6 , which is slightly acidic. The term $\mathrm{pH}$ is a measure of acidity or alkalinity and ranges from 0 to 14 . A pH measurement of 7 is regarded as neutral. Measurements below 7 indicate increased acidity, while those above indicate increased alkalinity.

\begin{abstract}
Allowance for Funds Used During Construction (AFUDC): A noncash item representing the estimated composite interest costs of debt and a return on equity funds used to finance construction. The allowance is capitalized in the property accounts and included in income.
\end{abstract}

Ampere: The unit of measurement of electrical current produced in a circuit by 1 volt acting through a resistance of $1 \mathrm{ohm}$. (See Current, Ohm, Volt.)

Anthracite: Anthracite, or hard coal, is the highest rank of economically useable coal. It is jet black with a high luster. The moisture content generally is less than 15 percent. Arthracite contains approximately 22 to 28 million Btu per ton as received and averages about 25 million Btu per ton. Its ignition temperature is approximately 925 to 970 degrees Fahrenheit. Virtually all of the anthracite mined is from northeastern Pennsylvania. It is used mostly for space heating and generating electricity.

Ash: Impurities consisting of silica, iron, alumina, and other incombustible matter which are contained in coal. Ash increases the weight of coal, adds to the cost of handling, and can affect the burning characteristics. Ash content is measured as a percent by weight of coal on an "as received" or a "dry" (moisture-free, usually part of a latoratory analysis) basis.

Asset: An economic resource that is tangible or intangible and which is expected to provide benefits to a business.

Available but not Needed Capability: Net capability of main generating units that are operable but not considered necessary to carry load, and cannot be connected to load within 30 minutes. (See Capability, Standby Facility.)

Bbl: The abbreviation for barrel. (See Barrel.)

Bcf: The abbreviation for one billion cubic feet.

Barrel: A volumetric unit of measure for crude oil and petroleum products equivalent to 42 U.S. galions.

Base Bill: A charge calculated by taking the rate from the appropriate electric rate schedule and applying it to the level of consumption. (See Electric Rate Schedule.)

Baseload: The minimum amount of electric power delivered or required over a given period of time at a steady state. (See Baseload Plant.)

Baseload Capacity: The generating equipment normally operated to serve loads on an around-the-clock basis. (See Baseload, Baseload Plant.)

Baseload Plant: A plant, usually housing highefficiency steam-electric units, which is normally operated to take all or part of the minimum load of a system, and which consequently produces electricity at an essentially constant rate and runs continuousiy. These units are operated to maximize system mechan- 
ical and thermal efficiency and minimize system operating costs. (See Baseload.)

Bituminous Cual: Bituminous coal, or soft coal, is the mosi common coal. It is dense, black, often with welldefined bands of bright and dull material. Its moisture content usually is less than 20 percent. The heating value ranges from 19 to 30 million Btu per ton as received and averages about 24 million Btu per ton. The ignition temperature ranges from about 700 to almost 900 degrees Fahrenheit. Bituminous coal is mined chiefly in the Appalachian and Interior coal fields. It is used for generating electricity, making coke, and space heating.

Boiler: A device for generating steam for power, processing, or heating purposes; or hot water for heating purposes or hot water supply. Heat from an external combustion source is transmitted to a fluid contained within the tubes found in the boiler shell. This fluid is delivered to an end-use at a desired pressure, temperature and quality. (See Prime Mover.)

Btu (British Thermal Unit); A standard unit for measuring the quantity of heat energy equal to the quantity of heat required to raise the temperature of 1 pound of water by 1 degree Fahrenheit.

Capability: The maximum load that a generating unit, generating station, or other electrical apparatus can carry under specified conditions for a given period of time without exceeding approved limits of temperature and stress.

Capacity: The amount of electric power delivered or required for which a generator, turbine, transformer, transmission circuit, station, or system is rated by the manufacturer. (See Generator Nameplate Capacity.)

Capacity Charge: An element in a two-part pricing method used in capacity transactions (energy charge is the other element). The capacity (demand) charge is assessed on the amount of capacity being purchased. Also called Demand Charge.

Capital (Financial): The line items on the right side of a balance sheet, which include debt, preferred stock, and common equity. A net increase in assets must be financed hy an increase in one or more forms of capital.
Census Divisions: The nine geographic divisions of the United States established by the Bureau of the Census, U.S. Department of Commerce for statistical analysis. The boundaries of Census divisions cc ncide with State boundaries. The Pacific Division is subdivided into the Pacific Contiguous and Pacific Noncontiguous areas.

Circuit: A conductor or a system of conductors through which electric current flows. (See Current.)

Coal: A black or brownish-black solid combustible substance formed by the partial decomposition of vegetable matter without access to air. The rank of coal, which includes anthracite, bituminous coal, subbituminous coal, and lignite, is based on fixed carbon, volatile matter, and heating value. Coal rank indicates the progressive alteration from lignite to anthracite. Lignite contains approximately 9 to $17 \mathrm{mil}$ lion Btu per ton. The contents of subbituminous and bituminous coal range from 16 to 24 million Btu per ton and from 19 to 30 million Btu per ton, respectively. Anthracite contains approximately 22 to 28 million Btu per ton. (See anthracite, bituminous coal, subbituminous coal, lignite)

Cogenerator: A generating facility that produces electricity and another form of useful thermal energy (such as heat or steam), used for industrial, commercial, heating, or cooling purposes.

Coincident Demand: The sum of two or more demands that ocsur in the same time interval. (See Demand.)

Coincident Peak Load: The sum of two or more peak loads that occur in the same time interval. (See Peak Load.)

Combined Cycle: An electric generating technology in which electricity is produced from otherwise lost waste heat exiting from one or more gas (combustion) turbines. The exiting heat is routed to a conventional boiler or to a heat recovery steam generator for utilization by a steam turbine in the production of electricity. This process increases the efficiency of the electric generating unit.

Combined Pumped-Storage Plant: A pumped-storage hydroclectric powior planit that uses bơth pumped water and natural streamflow to produce electricity. 
Commercial Operation: Commercial operation begins when control of the loading of the generator is turned over to the system dispatcher.

Commercial Retail Prices of Electricity: The price of electricity supplied to commercial or business establishments such as stores, offices, restaurants, and garages for lighting and other power purposes.

Commercial Sector: The commercial sector is generally defined as nonmanufacturing business establishments, including hotels, motels, restaurants, wholesale businesses, retail stores, and health, social, and educational institutions. Utilities may classify commercial service as including all consumers whose demand or annual use exceeds some specified limit. The limit may be set by the utility based on the rate schedule of the utility.

Construction Work In Progress (CWIP): The balance shown on a utility's balance sheet for construction work not yet completed but in progress. This balance line item may or may not be included in the rate base.

Consumption (Fuel): The amount of fuel used for gross generation, providing standby service and startup and/or flame stabilization. (See Fuel.)

Contract Cost: Price of fuels marketed on a contract basis covering a period of 1 or more years. Contract prices reflect market conditions at the time the contract was negotiated and therefore remain constant throughout the life of the contract or are adjusted through escalation clauses. Generally, contract prices do not fluctuate widely.

Contract Receipts: Purchases based on a negotiated agreement that generally covers a period of 1 or more years.

Cooperative (Cooperatively Owned Electric Utility): A group organized under the law into a utility company that will generate, transmit, and/or distribute supplies of electric energy to a specified area not being serviced by another utility. Such ventures are generally exempt from the Federal income tax laws. Most cooperatives have initially been financed by the U.S. Department of Agriculture's Kural Electrification Administration. (See Electric Power Industry.)
Cost: The amount paid to acquire resources such as plant and equipment, fuel, or labor servises.

Current (Electric): A flow of electrons in an electrical conductor. The strength or rate of movement of the electricity is measured in amperes. (See Ampere, Ohm, Volt.)

Demand (Electric): The rate at which electric energy is delivered to or by a system, part of a system, or piece of equipment, at a given instant or averaged over any designated period of time.

Distillate Fuel Oil: A general classification for one of the petroleum fractions produced in conventional distillation operations. It is used primarily for space heating, on-and-off-highway diesel engine fuel (including railroad engine fuel and fuel for agriculture machinery), and electric power generation. Included are products known as No. 1, No. 2, and No. 4 fuel oils; No. 1, No. 2, and No. 4 diesel fuels.

Distribution System: The portion of an electric system that is dedicated to delivering electric energy to an end-user. (See System (Electric), Transmission.)

Diversity Exchange: An exchange of capacity or energy, or both, between systems whose peak loads occur at different times.

Electric Plant: A facility containing prime movers, electric generators, and auxiliary equipment for converting mechanical, chemical, and/or fission energy into electric energy.

Electric Rate Schedule: A statement of the electric rate and the terms and conditions governing its application, including attendant contract terms and conditions that have been accepted by a regulatory body with appropriate oversight authority.

Electric Utility: An enterprise that is engaged in the generation, transmission, or distribution of electric energy primarily for use by the public and is the major power supplier within a designated service area. Electric utilities include publicly owned; cooperatively owned; and government-owned (municipals, Federal agencies, State projects, and public power districts) systems. 
Energy: The capacity for doing work as measured by the capability of doing work (potential energy) or the conversion of this capability to motion (kinetic energy). Energy has several forms, some of which are easily convertible and can be changed to another form useful for work. Most of the world's convertible energy comes from fossil fuels that are burned to produce heat that is then used as a transfer medium to mechanical or other means in order to accomplish tasks. Electrical energy is usually measured in kilowatthours, while heat energy is usually measured in British thermal units. (See Energy Source.)

Energy Charge: That portion of the charge for electric service based upon the electric energy $(\mathrm{kWh})$ consumed or billed.

Energy Deliveries: Energy generated by one electric utility system and clelivered to another system through one or more transmission lines. (See Wheeling Service.)

Energy Receipts: Energy generated by one electric utility system and received by another system through one or more transmission lines. (See Wheeling Service.)

Energy Source: The primary source that provides the power that is converted to electricity through chemical, mechanical, or other means. Energy sources include coal, petroleum and petroleum products, gas, water, uranium, wind, sunlight, geothermal, and other sources.

Equity Capital: The sum of capital from retained earnings and the issuance of stocks.

Expenditure: The incurrence of a liability to obtain an asset or service.

Facility: A location at which prime movers, electric generators, and/or equipment for converting mechanical, chemical, and/or nuclear energy into electric energy are situated. A facility may contain more than one type of prime mover. For cogenerators, the facility includes the industrial or commercial process.

Federal Energy Regulatory Commission (FERC): FERC is a quasi-independent regulatory agency within ihe Deparimeni of Energy having jurisdiction over interstate electricity sales, wholesale electric rates, hydroelectric licensing, natural gas pricing, oil pipeline rates, and gas pipeline certification.

Federal Power Act: Enacted in 1920, and amended in 1935, the Act consists of three parts. The first part incorporated the Federal Water Power Act administered by the former Federal Power Commission, whose activities were confined almost entirely to lirensing non-Federal hydroelectric projects. Parts II and III were added with the passage of the Public Utility Act. These parts extended the Act's jurisdiction to include regulating the interstate transmission of electrical energy and rates for its sale as wholesale in interstate commerce. The Federal Energy Regulatory Cormmission is now charged with the administration of this law. (See Federal Energy Regulatory Commission).

Federal Power Commission: The predecessor agency of the Federal Energy Regulatory Commission. The Federal Power Commission was created by an Act of Congress under the Federal Water Power Act on June 10,1920 . It was charged originally with regulating the electric power and natural gas industries. It was abolished on September 30, 1977, when the Department of Energy was created. Its functions were divided between the Department of Energy and the Federal Energy Regulatory Con mission. (See Federal Energy Regulatory Commission.)

FERC: The Federal Energy Regulatory Commission.

FERC Form 1: The "The Annual Report of Major Electric Utilities, Licensees and Others," collects financial and operational information from major privately owned electric utilities, licensees and others subject to the jurisdiction of the Federal Energy Reg. ulatory Commission. Approximately 180 electric utilities submit this form based on sales and transmission service (for each of the 3 previous calendar years) exceeding one of the following thresholds: 1 million megawatthours of total annual sales, 100 megawatthours of annual sales for resale, 500 megawathours of annual gross interchange out, or 500 megawatthours of wheeling for others (deliveries plus losses).

FERC Form 423: The "Monthly Report of Cost and Quality of Fuels for Electric Plants," collects data on the cost, quality, and sources of fuel. Approximately 225 electric utilities report tor each plant having a 
fossil-fueled steam-electric nameplate capacity of 50 or more megawatts.

Firm Gas: Gas sold on a continuous and generally long-term contract.

Flue Gas Particulate Collectors: Equipment used to remove fly ash from the combustion gases of a boiler plant before discharge to the atmosphere. Particulate collectors include elecirostatic precipitators, mechanical collectors (cyclones), fabric filters (baghouses), and wet scrubbers.

Flue Gas Desulfurization Unit (Scrubber): Equipment used to remove sulfur oxides from the combustion gases of a boiler plant before discharge to the atmosphere. Chemicals such as lime are used as the scrubbing media.

Forced Outage: The shutdown of a generating unit, transmission line or other facility, for emergency reasons or a condition in which the generating equipment is unavailable for load due to unanticipated breakdown.

Form EIA-759: The "Monthly Power Plant Report," collects monthly data from all electric utilities on net generation, consumption of coal, oil, and gas, and endof-month stocks of coal and oil for each plant by prime mover and fuel-type combination. Approximately 800 electric utilities report. Form EIA-759 replaced the FPC Form 4 in January 1982.

Form EIA-767: The "Steam Electric Plant Operation and Design Report," collects data annually on plant operations and equipment design including boiler, generator, cooling system flue gas desulfurization, flue gas particulate collector, and stack data. Respondents are required to report for all plants with a fossil-fueled steam-electric nameplate capacity of 10 or more megawatts. Approximately 900 planis report. Form EIA.767 replaced the FPC Form 67 in 1982.

Form EIA-826: The "Monthly Electric Utility Saies and Revenue Report with State Distributions," collects monthly data on customers, sales, revenues, sales for resale, and other financial items. Approximately 225 clcotio utilitics report.
Form EIA-860: The "The Annual Electric Generator Report," collects data from electric utilities in the United States on the status of electric power plants and associated equipment in operation within 10 years of the reporting year. Generator-specific data are reported by approximately 900 electric utilities.

Form EIA-861: The "The Annual Electric Utility Report," collects data on the source and disposition of energy, including net purchases, exchanges and wheeling, energy used by the electric department, energy furnished without charge, and energy losses, revenue, retail sales, sales for resale, and nonutility power production data. Form EIA-861 is a mandatory collection of data, filed annually by approximately 3,250 electric utilities in the United States, its territories, and Puerto Rico.

Fossil Fuel: Any naturally occurring organic fuel, such as petroleum, coal, and natural gas.

Fossil Fuel Plant: A plant using coal, petroleum, or gas as its source of energy.

Fuel: Any substance that can be burned to produce heat; also, materials that can be fissioned in a chain reaction to produce heat.

Fuel Expenses: These costs include the fuel used in the production of steam or driving another prime mover for the generation of electricity. Other associated expenses include unloading the shipped fuel, and all handling of the fuel up to the point where it enters the first bunker, hopper, bucket, tank, or holder in the boiler-house structure.

Full-Forced Outage: The net capability of main generating units that is unavailable for load for emergency reasons. (See Forced Ourage, Standby Facility.)

Gas: (See Natural Gas.)

Gas Turbine Plant: A plant in which the prime mover is a gas turbine. A gas turbine consists typically of an axial-flow air compressor, one or more combustion chambers where liquid or gaseous fuel is burned and the hot gases are passed to the turbine; where the hot gases expand to drive the generator and then are used to run the compressor. 
Generating Unit: Any combination of physically connected generator(s), reactor(s), boiler(s), combustion turbine(s), or other prime mover(s) operated together to produce electric power.

Generation: The process of producing electric energy by transforming other forms of energy; also, the amount of electric energy produced. (See Electric Plant, Energy.)

Generator: A machine that converts mechanical energy into electrical energy.

Generator Nameplate Capacity: The full-load continuous rating of a generator, prime mover, or other electrical power production equipment under specified conditions as designated by the manufacture. Installed generator nameplate rating is usually indicated on a nameplate physically attached to the generator.

Geothermal Plant: A plant in which the prime mover is a steam turbine. The turbine is driven either by steam produced from hot water or by natural steam that derives its energy from heat found in rocks or fluids at various depths beneath the surface of the earth. The energy is extracted by drilling and/or pumping.

Gigawatt (GW): One billion watts. (See Watt.)

Gigawatthour (GWh): One billion watthours. (See W'atthour.)

Greenhouse Effect: The increasing mean global surface temperature of the earth caused by gases in the atmosphere (including carbon dioxide, methane, nitrous oxide, ozone, and chlorofluorocarbons). The greenhouse effect allows solar radiation to penetrate but absorbs the infrared radiation returning to space.

Grid: The layout of an electrical distribution system. (See Transmission, Transmission System, Electric)

Gross Generation: The total amount of electric energy produced by a generating facility as measured at the generator terminals. (See Generation, Electric Plant.)
Heavy Oil: The fuel oils remaining after the lighter oils have been distilled off during the refining process. Except for start-up and flame stabilization, virtually all petroleum used in steam plants is heavy oil.

Hydroelectric Plant: A plant in which the turbine generators are driven by falling water.

Industrial Retail Prices of Electricity: Price of the electricity supplied to industrial establishments such as automotive and steel producing industries.

Industrial Sector (Electricity): The industrial sector is generally defined as including manufacturing, construction, mining, agriculture, fishing, and forestry establishments Standard Industrial classified under Standard Industrial Classification (SIC) codes 01-39. The utility may classify industrial service using the SIC codes, or based on demand or annual usage exceeding some specified limit. The limit may be set by the utility based on its own rate schedule. Sales for consumers (i.e. farms and irrigation) that the utility has no system for separating into residential, commercial, and industrial classifications, should be class îed based on the classification of consumer their rate most closely resembles. If there is no rate schedule distinction, utilities may define industrial consumers as those having a demand equal to or greater than 1,000 kilowatts.

Interdepartmental Service: Electricity supplied to departments of the electric utility company other than the electric generating department.

Intermediate Load: The range from base load to a point between base load and maximum peak. This point, which is dependent on the load curve, may be the midpoint, a percent of the peak load, or the load over a specified time period.

Internal Combustion Plant: A plant in which the prime mover is an internal combustion engine. An internal combustion engine has one or more cylinders in which the process of combustion takes place, converting energy released from the rapid burning of a fuel-air mixture into mechanical energy. Diesel or gasfired engines are the principal types used in electric plants. The plant is usually operated during periods of high demand for electricity. 
Interruptible Gas: Gas sold to customers with a provision that permits curtailment or cessation of service and at the discretion of the distributing company under certain circumstances as specified in the service contract.

Kilowatt (kW): One thousand watts. (See Watt.)

Kilowatthour (kWh): One thousand watthours. (See Watthour.)

Leverage Ratio: The ratio of total debt to total assets.

Liability: An amount payable in dollars or by future services to be rendered.

Light Oil: Lighter fuel oils distilled off during the refining process. Virtually all petroleum used in internal combustion and gas-turbine engines is light oil.

Lignite: Lignite, the lowest rank of coal, is brownish black and has a high moisture content, sometimes as high as 45 percent. It tends to disintegrate when exposed to the weather. The heat content of lignite ranges from 9 to 17 million Btu per ton as received and averages about 14 million Btu per ton. The ignition temperature is approximately 600 degrees Fahrenheit. Lignite is mined in California, Louisiana, Montana, North Dakota, and Texas, and is used mainly to generate electricity in power plants that are relatively close to the mines.

Load (Electric): The amount of electric power delivered or required at any specific point or points on a system. The requirement originates at the energyconsuming equipment of the customers.

Maximum Demand: The greatest of all dernands of the load that has occurred within a specified period of time.

Mcf: One thousand cubic feet.

Megawatt (MW): One million watts. (See Watt.)

Megawatthour (MWh): One million watthours. (See Watthour.)
MMcf: One million cubic feet.

Natural Gas: A naturally occurring mixture of hydrocarbon and nonhydrocarbon gases found in porous geological formations beneath the earth's surface, often in association with petroleum. The principal constituent is methane.

Net Generation: Gross generation less plant use, measured at the high-voltage terminals of the station's step-up transformer. The energy required for pumping at pumped-storage plants is regarded as plant use and must be deducted from the gross generation. (See Generation, Electric Plant.)

Net Summer Capability: The steady hourly output which generating equipment is expected to supply to system load exclusive of auxiliary power as demonstrated by tests at the time during summer peak demand.

Net Winter Capability: The steady hourly output which generating equipment is expected to supply to system load exclusive of auxiliary power as demonstrated by test at the time of winter peak demand.

Noncoincident Peak Load: The sum of two or more peak loads on individual systems that do not occur in the same time interval. Meaningful only when considering loads within a limited period of time, such as a day, week, month, a heating or cooling season, and usually for not more than 1 year.

Nonutility Power Producer: An enterprise that has electric generating capacity and is not an electric utility. Nonutility power producers include qualifying cogenerators, qualifying small power producers, and other nonutility generators (including independent power producers) without a designated franchised service area, and which are not otherwise required to file forms listed in the Code of Federal Regulations, Title 18, Part 141.

North American Electric Reliability Council (NERC): A council formed in 1968 by the electric utility industry to promote the reliability and adequacy of bulk power supply in the electric utility systems of North America. NERC consists of nine regional reliability councils and encompasses essentially all the power systems of the contiguous United States, Canada, and some in Mexico. The NERC Regions are: 
ECAR - East Central Area Reliability Coordination Agreement

ERCOT - Electric Reliability Council of Texas

MAIN - Mid-America Interconnected Network

MAAC - Mid-Atlantic Area Council

MAF'P - Mid-Continent Area Power Pool

NPCE - Northeast Power Coordinating Council

SERC - Southeastern Electric Reliability Council

SPP - Southwest Power Pool

WSCC - Western Systems Coordinating Council

Nuclear Fuel: Fissicnable materials that have been enriched to such a composition that when placed in a nuclear reactor will support a self-sustaining fission chain reaction, producing heat in a controlled manner for process use.

Nuclear Power Plant: A plant in which the prime mover is a steam turbine. The steam used to drive the turbine is produced by a heat transfer from the reactor vessel during the period when the nuclear fuel is undergoing fission.

Off-Peak Gas: Gas which is to be delivered and taken on demand when demand is not at its peak. (See Spot Purchases.)

Ohm: The unit of measurement of electrical resistance. The resistance of a circuit in which a potential di. ${ }^{\text {se }} \mathrm{r}$ ence of one volt produces a current of one ampere. (See Ampere, Current, Volt.)

Operable: A unit is operable when it is available to provide power to the grid. For a nuclear unit, this is when it receives its full power amendment to its operating license from the Nuclear Regulatory Commission.

Other Service to Public Authorities: Electricity supplied to municipalities or divisions or agencies of State or Federal governments, under special contracts or agreements or service classifications applicable only to public authorities.
Outage: The period during which a generating unit, transmission line, or other facility is out of service. (See Forced Outage, Scheduled Outage.)

Peak Load: The maximum load during a specified period of time.

Peak Load Plant: A plant usually housing old, lowefficiency steam units; gas turbines; diesels; or pumpedstorage hydroelectric equipment normally used during the peak-load periods.

Peaking Capacity: Crspacity of generating equipment normally reserved for operation during the hours of highest daily, weekly, or seasonal loads. Some generating equipment may be operated at certain times as peaking capacity and at other times to serve loads on an around-the-clock basis. (See Peak Load.)

Percent Difference: The relative change in a quantity over a specified time period. It is calculated as follows: The current value number has the previous value number subtracted from it, and this new number is divided by the absolute value of the previous value number; then this new number is multiplied by 100 .

Petroleum: A mixture of hydrocarbons existing in the liquid state found in nalural underground reservoirs, often associated with gas. Petrolsum includes Fuel Oil 2, 4, 5, 6, topped crude, kerosene, and jet fuel. (See Petroleum (Crude Oil.))

Petroleum Coke: A residue, high in carbon content and low in hydrogen, that is the final product of thermal decomposition in the condensation process in cracking. This product is reported as marketable coke or catalyst coke. The conversion factor is 5 barrels (of 42 U.S. gallons each) per short ton.

Petroleum (Crude Oil): A naturally occurring, oily, flammable liquid composed principally of hydrocarbons. Crude oil is occasionally found in springs or pools but usually is drilled from wells beneath the earth's surface.

Plant Use: The electric energy used in the operation of a plant. Included in this definition is the energy required for pumping at pump-storage plants. 
Plant-Use Electricity: The electric energy used in the operation of a plant. This energy total is subtracted from the gross energy production of the plant; for reporting purposes the plant energy production is then reported as a net figure. The energy required for pumping at pumped-storage plants is by definition subtracted, and the energy production for these plants is then reported as a net figure. (See Combined Pumped-Storage Plant, Pumped-Storage Hydroelectric Plant, Pure Pumped-Storage Hydroelectric Plant.)

Power: The rate at which energy is transferred. Electrical energy is usuall!' measured in watts. Also used for a measurement of ca racity. (See Capacity, Energy, Watt.)

Power Pool: An association of two or more interconnected electric systems to provide better system reliability and efficiencies.

Price: The amount of money or consideration-in-kind for which a service is bought, sold, or offered for sale.

Prime Mover: The motive force, eg. steam, engine, turbine or water that drives an electric generator.

Profit: The income remaining after all business expenses are paid.

Public Street and Highway Lighting Service: Electricity supplied for the purpose of lighting streets, highways, parks, and other public places; or for traffic or other signal system service, for municipalities, or other divis:ons or agencies of State or Federal governments.

Pumped-Storage Hydroelectric Plant: A plant that usually generates electric energy during peak-load periods by using water previously pumped into an elevated storage reservoir during off-peak periods when excess generating capacity is available to do so. When additional generating capacity is needed, the water can be released from the reservoir through a conduit to turbine generators located in a power plant at a lower level.

Purchased Power Adjustment: A clause in a rate schedule that provides for adjustments to the bill when energy from another electric system is acquired and its cost varies from a specified unit base amount.
Pure Pumped-Storage Hydroelectric Plant: A plant that produces power only from water that has previously been pumped to an upper reservoir.

Qualifying Facility: A cogeneration facility or small power production facility that is not more than 50 percent owned by electric utilities and meets certain standards as defined under Section 201 of the Public Utilities Regulatory Policies Act of 1978.

Railroads and Railways Service: Electricity suppiied to railroads and interurban and street railways, for general railroad use, including the propulsion of cars or locomotives, where such electricity is supplied under separate and distinct rate schedules.

Rate Base: The value of property upon which a utility is permitted to earn a specified rate of return as established by a regulatory authority. The rate base generally represents the value of property used by the utility in providing service and may be calculated by any one or a combination of the following accounting methods: fair value, prudent investment, reproduction cost, or original cost. Depending on which method is used, the rate base includes cash working capital, materials and supplies, and deductions for accumulated provisions for depreciation, contributions in aid of construction, customer advances for construction, accumulated deferred income taxes, and accumulated deferred investment tax credits.

Ratemaking Authority: A utility commission's legal authority to fix, modify, approve or disapprove rates as determined by the powers given the commission by a State or Federal legislature. (See Regulation.)

Receipts: Purchases of fuel.

Regulation: A utility commission carries out its regulatory functions through rulemaking and adjudication. Under rulemaking, the utility commission may propose a general rule or regulation change. By law, it must issue a notice of the proposed rule and a request for comments is also made; the Federal Energy Regulatory Commission publishes this in the Federal Register. The final decision must be published. A utility commission may also work on a case-by-case basis from submissions from regulated companies or others. Objections to a proposal may come from the FERC or intervenors, in which case the proposal must be presented to a hearing presided over by an administrative law judge. The judge's decision may be 
adopted, modified, or reversed by the utility commissioners in which case those involved can petition for a rehearing, and may appeal a decision through the courts to the U.S. Supreme Court. (See Ratemaking Authority.)

Reserve Margin (Operating): The amount of unused available capability of an electric power system at peak load for a utility system as a percentage of total capability.

Residential Retail Prices of Electricity: Price of electricity supplied to residential customers for lighting, refrigeration, cooling, water heating, and other domestic uses.

Residential Sector: The residential sector includes private household establishments which consume energy primarily for space heating, water heating, air conditioning, lighting, refrigeration, cooking, and clothes drying. The classification of an individual consumer's account, where the use is both residential and commerciai, is based on principal use. Apartment houses are included.

Residual Fuel Oil: The topped crude of refinery operation; includes No. 5 and No. 6 fuel oils as defined in ASTM Specification D396 and Federal Specification VV-F-815C, Navy Special fuel oil as defined in Military Specification MIL-F-859E including Amendment 2 (NATO Symbol F-77), and Bunker C fuel oil. Residual fuel oil is used for the production of electric power, space heating, vessel bunkering, and various industrial purposes. Imports of residual fuel oil include "Imported Crude Oil Burned as Fuel."

Revenue: An increase in the assets of an organization or the decrease in liabilities during an accounting period, primarily from the organization's operating activities. This may include the sale of electricity, and earnings from interest, dividends, lease income and royalties. (See Asset and Liability)

Running and Quick-Start Capability: The net capability of generating units that carry load or have quickstart capability. In general, quick-start capability refers to generating units that can be available for load within a 30-mınute period. (See Capability, Load (Electric), Net Summer Capability.)
Sales: The amount of kilowatthours sold in a given period of time; usually grouped by classes of service such as residential, commercial, industrial, and other. Other sales include public street and highway lighting, other sales to public authorities, sales to railroads and sailways, and interdepartmental sales.

Sales for Resale: A type of wholesale sales covering energy supplied to other electric utilities, cooperatives, municipalities, and Federal and State electric agencies for resale to ultimate consumers. (See Wholesale Sales.)

Scheduled Outage: The shutdown of a generating unit, transmission line, or other facility, for inspection or maintenance, in accordance with an advance schedule. (See Forced Outage.)

Short Ton: A unit of weight equal to 2,000 pounds.

Spinning Reserve: That reserve generating capacity rurning at a zero load and synchronized to the electric system.

Spot Purchtises: A single shipment of fuel or volumes of fuel, purchased for delivery within 1 year. Spot purchases are often made by a user to fulfill a certain portion of energy requirements, to meet unanticipated energy needs, or to take advantage of low fuel prices.

Stability: The property of a system or element by virtue of which its output will ultimately attain a steady state. The amount of power that can be transferred from one machine to another following a disturbance. The stability of a power system is its ability to develop restoring forces equal to or greater than the disturbing forces so as to maintain a state of equilibrium.

Standard Industrial Classification (SIC): A set of codes developed by the Office of Management and Budget, which categorizes business into groups with similar economic activities.

Standby Facility: A facility that supports a utility system and is generally running under no-load. It is available to replace or supplement a facility normally in service. (See Standby Service, Outage.) 
Standby Service: Support service that is available as needed to supplement a customer, a utility system, or to another utility if a schedule or an agreement authorizes the transaction. The service is not regularly used. (See Standby Facility, Outage.)

Steam-Electric Plant (Conventional): A plant in which the prime mover is a steam turbine. The stean used to drive the turbine is produced in a boiler where fossil fuels are burned.

Stocks (Fuel): A supply of fuel accumulated for future use. This includes coal and fuel oil stocks at the plant site, in coal cars, tanks, or barges at the plant site, or at separate storage sites.

Subbituminous Coal: Subbituminous coal, or black lignite; is dull black and generally contains 20 to 30 percent moisture. The heat content of subbituminous coal ranges from 16 to 24 million Btu per ton as received and averages about 18 million Btu per ton. Subbituminous coal, mined in the western coal fields, is used for generating electricity and space heating.

Substation: Facility equipment that switches, changes, or reguidtes electric voltage. (See Transformer, Transmission.)

Switching Station: Facility equipment used to tie together two or more electric circuits through switches. The switches are selectively arranged to permit a circuit to be disconnected, or to change the electric connection between the circuits. (See Electric Plant, Substation, Transformer, Transmission.)

System (Electric): Physically connected generation, transmission, and distribution facilities operated as an integrated unit under one central management, or operating supervision.

Total Bill: An itemized statement of fees and charges that consists of the base bill plus State and local taxes and other charges. These charges can include environmental surcharges, franchise taxes, purchased power adjustments, fuel adjustments, and any other taxes passed onto the customer from State and local governments which are collected by the utility.

Transformer: An electrical device for changing the voltage of alternating current.
Transmission: The movement or transfer of electric energy over an interconnected group of lines and associated equipment between points of supply and points at which it is transformed for delivery to consumers, or is delivered to other electric systems. Transmissio is considered to end when the energy is transformed for distribution to the consumer. (See Current, Energy, Power, Watt.)

Transmission System (Electric): An interconnected group of electric transmission lines and associated equipment for moving or transferring electric energy in bulk between points of supply and points at which it is transformed for delivery over the distribution system lines to consumers, or is delivered to other electric systems. (See Transmission.)

Turbine: A machine for generating rotary mechanical power from the energy in a stream of fluid (such as water, steam, or hot gas). Turbines convert the kinetic energy of fluids to mechanical energy through the principles of impulse and reaction, or a mixture of the two.

Uniform System of Accounts: Prescribed financial rules and regulations established by the Federal Energy Regulatory Commission for utilities subject to its jurisdiction under the authority granted by the Federal Power Act.

Volt: The unit of measurement of electric force or pressure. The electrical force that, if steadily applied to a circuit with a resistance of one ohm, will produce a current of one ampere. (See Ampere, Current, Ohm.)

Voltage Reduction: Any intentional reduction of system voltage by three percent or greater for reasons of maintaining the continuity of service of the bulk electric power supply system.

Watt: The electrical unit of power. The rate of energy transfer equivalent to one ampere flowing under a pressure of one volt at unity power factor.

Watthour (Wh): An electrical energy unit of measure equal to 1 watt ff power supplied to, or taken from, an electric circuit steadily for 1 hour.

Wheeling Service: The movement of electricity from one system to another over transmission facilities of 
intervening systems. Wheeling service contracts can be established between two or more systems.
Wholesale Sales: Energy supplied to other electric utilities, cooperatives, municipalities, and Federal and State electric agencies for ro sale to ultimate consumers. 


\section{Your Entrée to}

Federal Statistics!

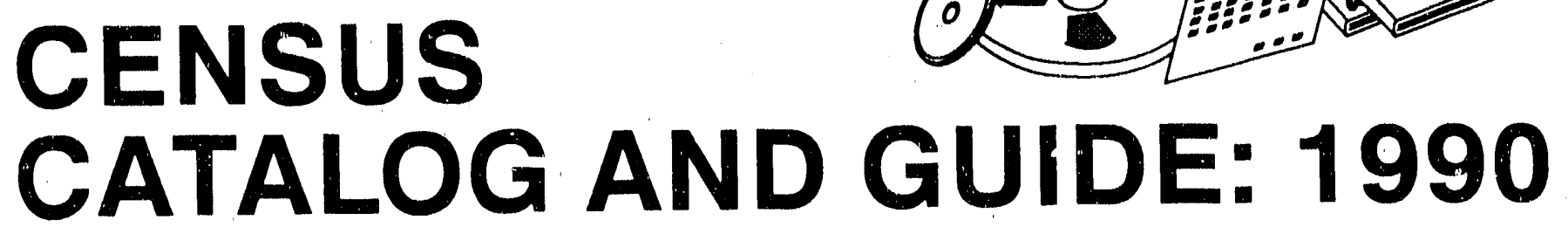

Helps you select from all the products

Every Census Bureau product issued mid-1988--1989:

Reports Microfiche Computertapes CD-ROM

Maps Floppy disks Online access

Key statistical publications from other Federal agencles (NEW!)

Features facts about each product

Topics Areas Dates Prices

Makes finding the right product easy

Extensive overview chapter Title index

Detailed subject index Guides to each statistical subject

Identifies sources of assistance

1,400 State Data Center organizations

200 Census Bureau specialists

1,500 depository libraries

\section{Contents}

General and Reference

Agriculture

Business

Construction and Housing

Foreign Trade

Geography

Governments

International

Manufacturing and Mineral Industries

Population

Transportation

1990 Census Product Plans (NEWI)

Sources of Assistance

Factfinder Guides

Other Federal statistical agencies

4 other directory lists

*6835

Superintendent of Documents Publication Order Form

Yes, please send me: copies of Census Catalog and Gulde: 1990 at $\$ 14$, S/N 003-024-07169-0.

1. The total cost of my order is $\$$ . (International customers please add 25\%.) All prices include regular domestic pnstage and handling and are good through $1 / 91$. After this date, please call Order and Information Desk at 202-783-3238 to verify prices.

Charge your order. It's easy!

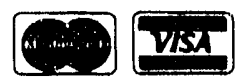

2. Please Type or Print

(Company or personal name)

(Additional address/attention line)

(Street address)

(City, State, ZIP Code)

(Daytime phone including area code)
3. Please Choose Method of Payment:

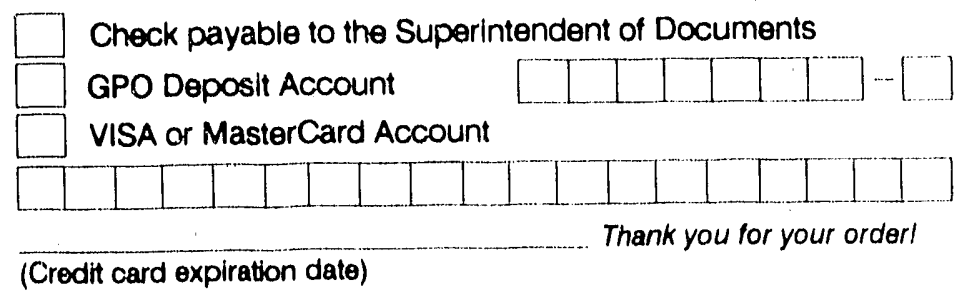

(Signature)

4. Mall To: Superintendent of Docurnerits, Government Printing Office, Washington, DC 20402-9325 

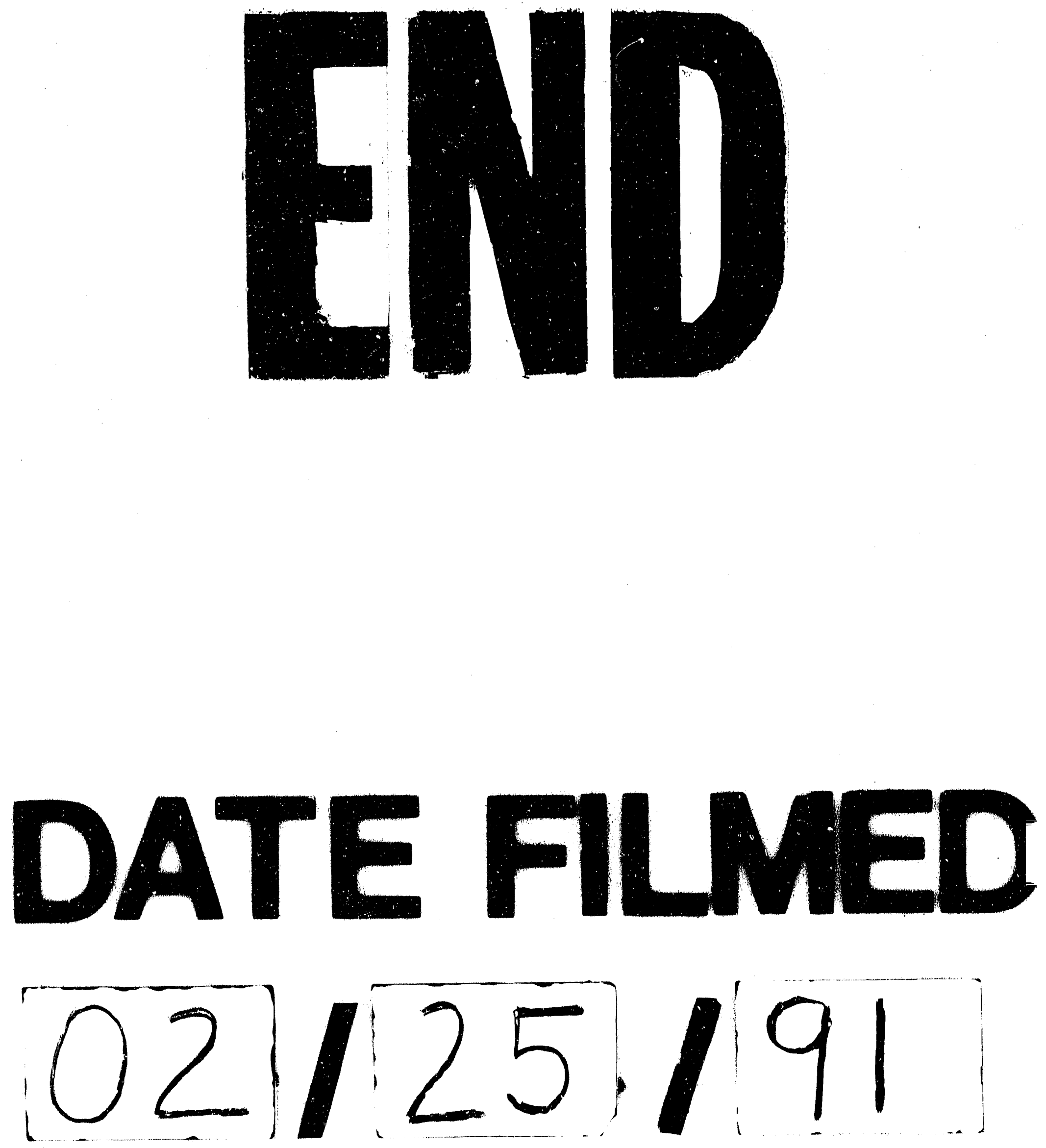
